\title{
Cortical patterning in syncytial embryos: the link between microtubules and actin cortex
}

\author{
Dissertation \\ for the award of the degree \\ " Doctor rerum naturalium" (Dr. rer. nat.) \\ of the Georg-August-Universität Göttingen \\ within the doctoral program Biology \\ of the Georg-August University School of Science (GAUSS)
}

\author{
submitted by \\ Long Li \\ from Hunan, China
}

Göttingen, 2019 


\section{Thesis Committee}

\section{Prof. Dr. J. Großhans}

Institute for Developmental Biochemistry, Medical School, University of Göttingen.

\section{Prof. Dr. G. Bucher}

Department of Evolutionary Developmental Genetics, University of Göttingen.

\section{Prof. Dr. A. Müller}

Department of Developmental genetics, Institute of Biology, University of Kassel

\section{Members of the Examination Board}

Reviewer: Prof. Dr. J. Großhans

Institute for Developmental Biochemistry, Medical School, University of Göttingen.

Second Reviewer: Prof. Dr. G. Bucher

Department of Evolutionary Developmental Genetics, University of Göttingen.

\section{Further members of the Examination Board}

\section{Prof. Dr. A. Müller}

Department of Developmental genetics, Institute of Biology, University of Kassel.

\section{Prof. Dr. S. Hoyer-Fender}

Johann-Friedrich-Blumenbach Institute for zoology and anthropology, University of Göttingen.

\section{Dr. J. C. Gross}

Institute for Developmental Biochemistry, Medical School, University of Göttingen.

\section{Dr. G. Vorbrüggen}

Max Planck Research Group of Molecular Cell Dynamics Cell Migration and Adhesion, Max Planck Institute for Biophysical Chemistry, Göttingen. 


\section{AFFIDAVIT}

I hereby declare that I prepared the doctoral thesis "Cortical patterning in syncytial embryos: the link between microtubules and actin cortex" on my own with no other sources and aids than quoted.

\section{Long Li}

Göttingen, 16.11.2019 


\section{Acknowledgements}

I would firstly like to thank my supervisor Prof. Jörg Großhans for providing me such an interesting topic during my doctoral study. I am very grateful for his patience, optimism for discussion and answering questions about my topic. I am very thankful for my thesis committee members, Prof. Bucher and Prof. Müller for giving me some suggestions and constructive criticisms about my topic during the thesis committee meetings. Secondly, I would like to thank my colleagues to provide a friendly atmosphere in the lab and great discussions during coffee break, I am very happy to work in this lab. I would like to thank Dr. Zhiyi Lv for helping me even before I came here and helpful discussion about the experiments. I am grateful to Dr. Deqing Kong and Dr. Shuling Yan for sharing experience about the experiment and life. I also would like to thank Dr. Maria Kriebel for trying to get me out of bored life. I am grateful for all the person who helped me during my doctoral study.

Last but not the least, I would like to thank my family members for their mental support and their encouragement during my study. 
1 Abstract

Part A Cortical patterning in syncytial embryos: the link between microtubules and actin cortex 2 Introduction

2.1 Cortical polarization and functions of cytoskeleton in cell polarity .................................... 5

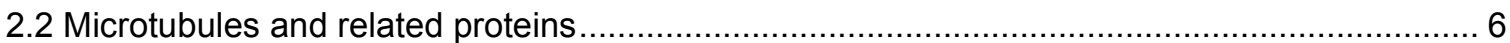

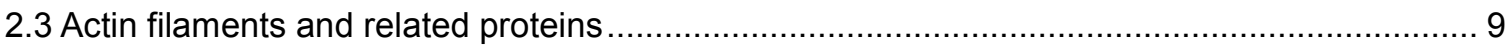

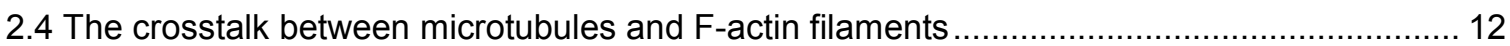

2.5 Cortical polarity in epithelial cells and C. elegans embryos.............................................. 13

2.6 The formation of different cortical domains and the polarity regulation during Drosophila early

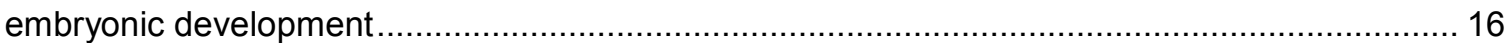

2.7 Aim of the study

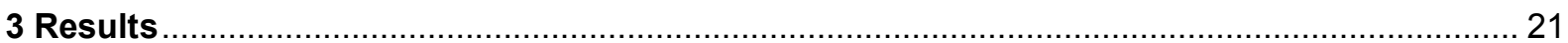

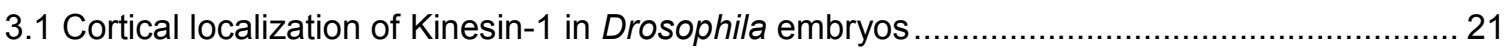

3.1.1 Kinesin-1 localizes to the cortex of embryos during syncytial and cellularization stages

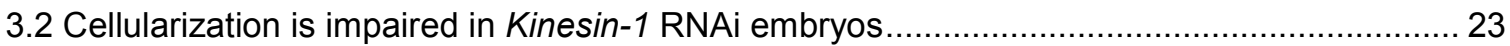

3.3 The cortical polarization is affected in Kinesin-1 RNAi embryos ............................................ 24

3.4 Centrosomes and microtubules are not affected in Kinesin-1 depleted embryos ................... 32

3.5 The differentiation of cap and intercap domains is not affected in Kinesin-1 RNAi embryos . 36

3.6 The organization of F-actin is altered in Kinesin-1 depleted embryos .................................. 38

3.6.1 Cpa-GFP clusters are mainly accumulated to the intercap domain during interphase. 43

3.6.2 The localization of Cpa-GFP clusters is disrupted in Kinesin-1 RNAi embryos ............ 50

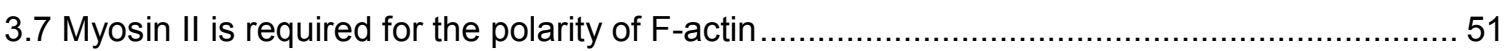

3.7.1 Myosin II is mislocalized at the intercap domain in Kinesin-1 RNAi embryos................ 51

3.7.2 The Cpa-GFP localization depends on Myosin II..................................................... 53

3.8 The Cpa-GFP clusters distribution is affected in dia mutant ............................................. 56

3.8.1 Dia is required for the Cpa localization in syncytial and cellularization stages ............. 58

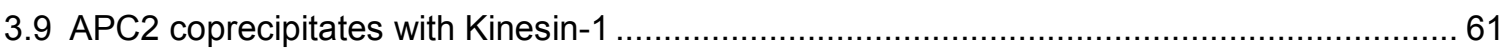

3.10 APC2 is required for the membrane ingression during cellularization, but it is not the linker

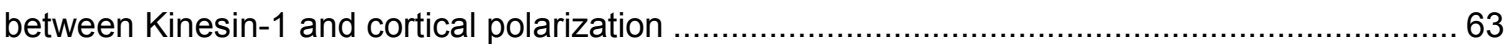

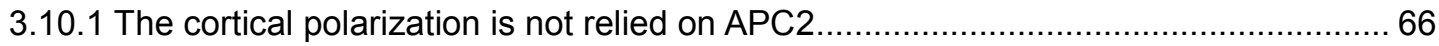

3.10.2 Accumulations of Slam and Amphiphysin at the furrow tip are disrupted in APC2 d40

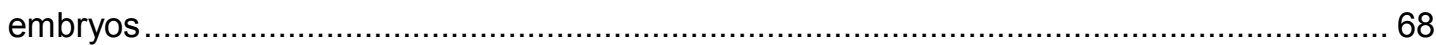

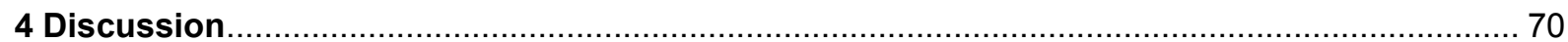

Part B Mapping of slam RNA sequence for Slam expression and RNA localization

5 Introduction: function and connection of slam mRNA and protein in Drosophila early

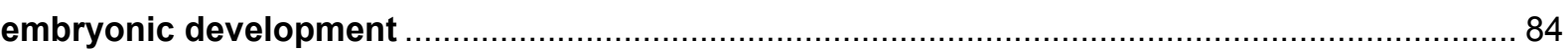

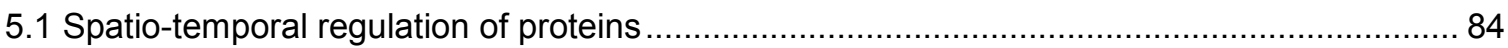

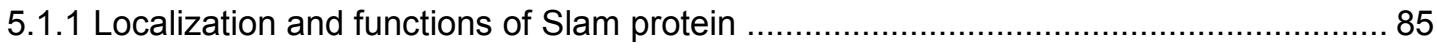

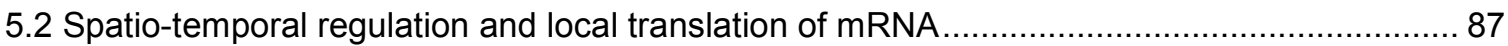

5.2.1 Spatio-temporal dynamics and the local translation of slam mRNA ........................... 89

5.2.2 The function of slam mRNA sequence in slam mRNA localization and Slam protein

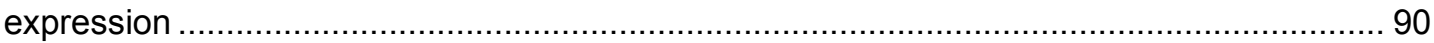

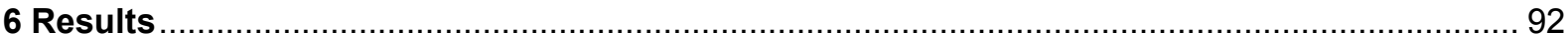

6.1 Noncoding functions of slam mRNA for RNA localization and translation ............................. 92

6.2 Mapping of the localization and translation elements in slam mRNA .................................... 94

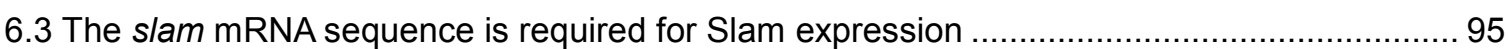

6.4 The slam mRNA sequence is required for slam mRNA localization ........................................ 99

7 Discussion 
8 Materials and Methods

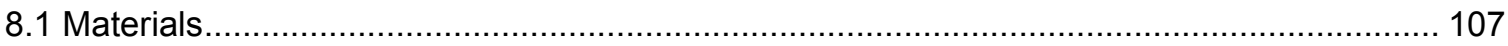

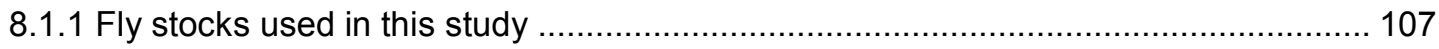

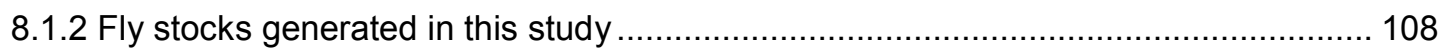

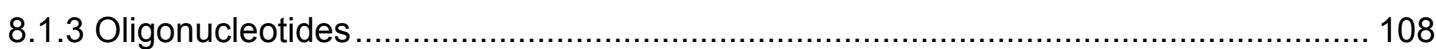

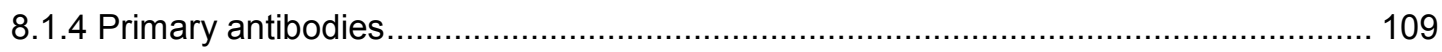

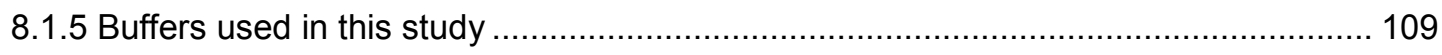

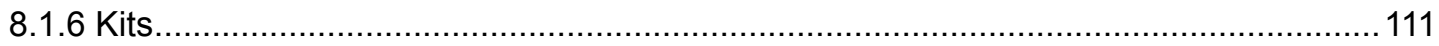

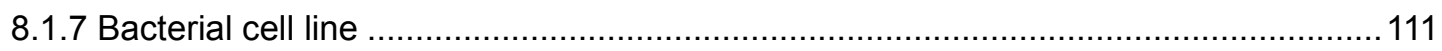

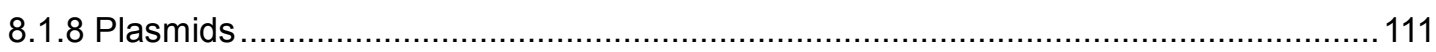

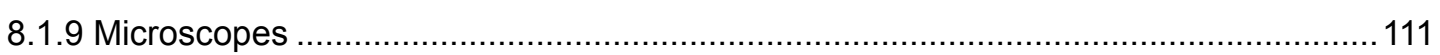

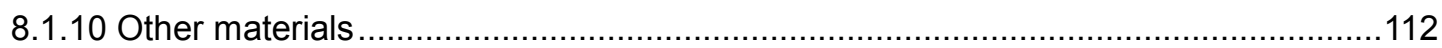

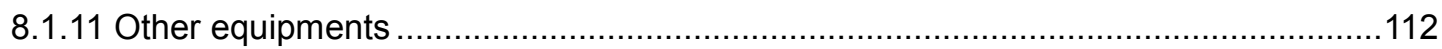

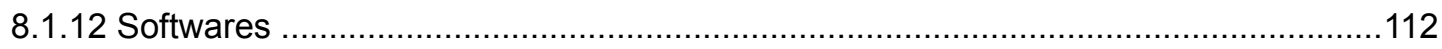

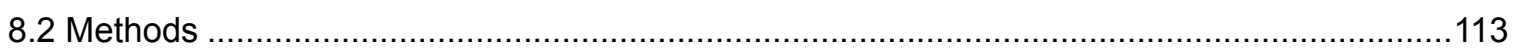

8.2.1 Fixation of Drosophila embryos (Heat fixation and Formaldehyde fixation) ................113

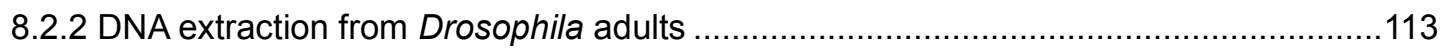

8.2.3 Cloning of different hybrid slam mRNA sequences.............................................114

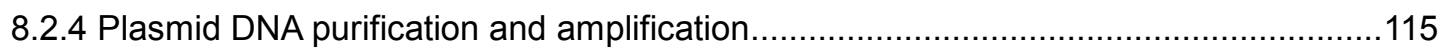

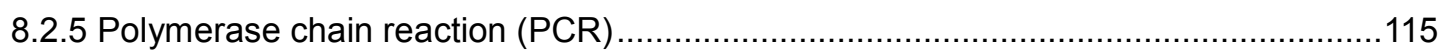

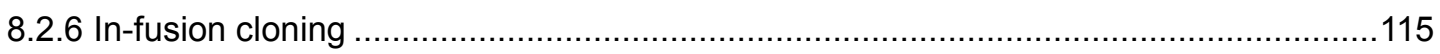

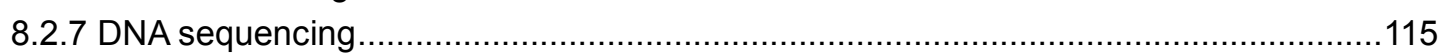

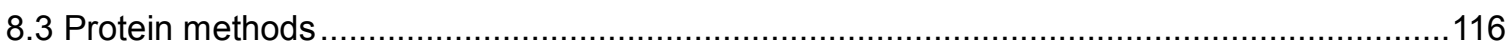

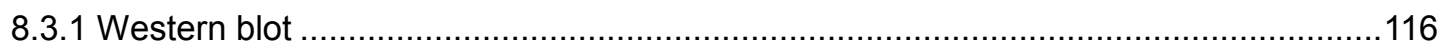

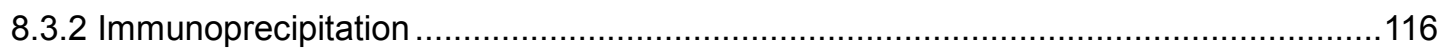

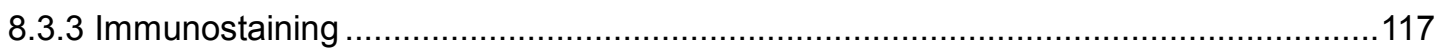

8.3.4 Rho kinase inhibitor Y-27632 injection and immunostaining ....................................117

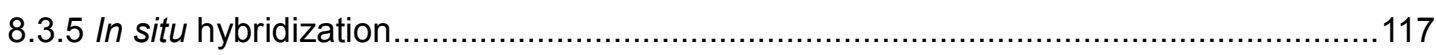

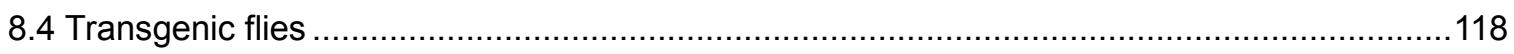

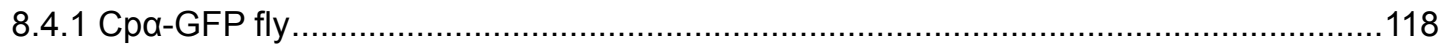

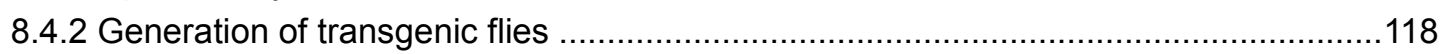

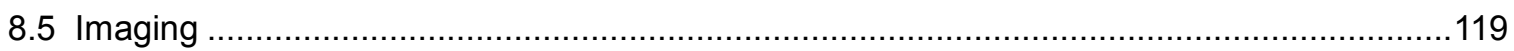

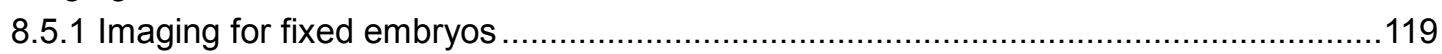

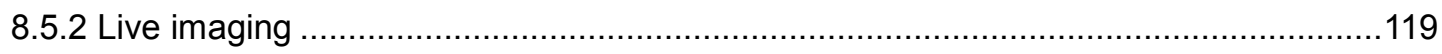

8.5.3 Fluorescence recovery after photobleaching (FRAP) experiment ............................119

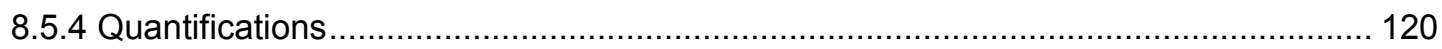

References

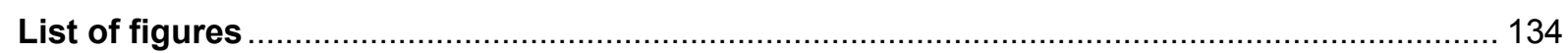

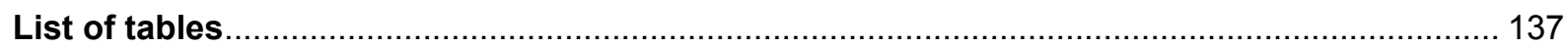

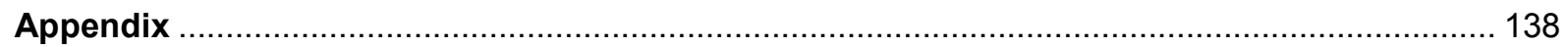

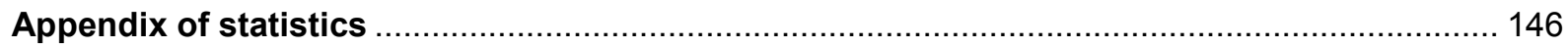

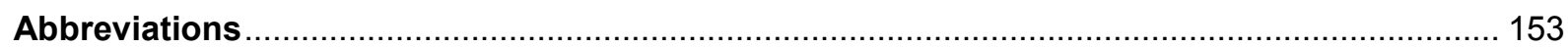




\section{Abstract}

Spatial and temporal regulation of cortical proteins on the cell membrane leads to cortical polarization of cells. After cortical polarization, the cell membrane can divide into different domains such as apical domain and basolateral domain. Cortical polarity is crucial for cell differentiation and function. For example, cell polarity is required for the formation of spatially restricted structures, like cell junctions in differentiated cells. Also cell polarity is important for the morphological complexity during embryonic development. In $C$. elegans embryos, the cell polarization can be found at one-cell stage. With anterior cortical flow created by actomyosin contraction, the cortex of $C$. elegans embryos separates into anterior side and posterior side. With proliferation and polarization, cells in C. elegans embryos start internalization and migration during gastrulation, embryos separate into ectodermal, endodermal and mesodermal compartments, this is required for organogenesis. The membrane polarization also significantly happens in Drosophila early embryonic development, the cortex of Drosophila embryos differentiates into apical, subapical, lateral, and basal domains during cellularization. Drosophila embryos finish 13 nuclear cycles in about $2 \mathrm{~h}$ at room temperature, following with embryo cellularization. With the membrane invagination during cellularization, Drosophila embryos divide into more than 6000 cells. Since the polarity of cortical domain is important for embryonic differentiation and development, it is vital to fully understand mechanisms of the cell polarization and functions of different proteins in cell polarization. As the cortex of Drosophila embryos differentiates into four different domains in about $3 \mathrm{~h}$, it is a good model to study the cortical polarization in early embryonic development.

The cytoskeleton includes microtubules, microfilaments and intermediate filaments, they are not only providing mechanical support, but are also essential for cortical polarization. Kinesin-1, as a microtubule-dependent motor protein, is required for cargos transport in different cellular processes, such as nuclear positioning, ooplasmic streaming, and cortical polarization. Previous report showed that Kinesin-1 depletion affects the cellularization in Drosophila embryos, the membrane invagination during cellularization is also disrupted in Kinesin-1 RNAi embryos, but mechanisms how Kineisn-1 influences cellularization are not so clear yet. Functions of microtubules and actin network in cell biology and biophysics have been studied 
for several decades, interactions between microtubules and actin network in core processes have been concerned. However, whether Kinesin-1 depletion affects the polarity of F-actin cap during the syncytial interphase of Drosophila embryos has not been investigated.

To understand how Kinesin-1 regulates the cell polarization during cellularization and how Kinesin-1 influences the organization of F-actin cap during the syncytial interphase, in this study, I utilized Drosophila Kinesin-1 RNAi embryos to checked the localization of cortical components during syncytial stage and cellularization. I also focused on the organization of F-actin cap in Kinesin-1 RNAi embryos. I found that the disruption of cellularization in Kinesin-1 depleted embryos is due to the mislocalization of cortical components, they are stuck at the surface of Kinesin-1 RNAi embryos during cellularization. However, dynamics of GFP-Slam in wild type and Kinesin-1 RNAi embryos are comparable during cellularization, centrosomes and recycling endosomes in Kinesin-1 RNAi embryos are also fine. Although the cortical polarization in Kinesin-1 RNAi embryos is comparable to wild type during the syncytial stage, the localization of Canoe and ELMO/Sponge complex is affected. Furthermore, live images and immunostainings of Capping a (Cpa) indicate that Kinesin-1 is essential for the localization of Cpa at the intercap domain. In Kinesin-1 RNAi embryos, not only the contraction but also the polarity of the F-actin cap are influenced. The accumulation of Cpa at the intercap domain is affected in Kinesin-1 RNAi embryos. Myosin II cannot accumulate to the intercap domain in Kinesin-1 RNAi embryos. By injecting ROCK inhibitor into Drosophila embryos, I found that the disruption of Myosin II affects the polarity of F-actin cap, the distribution of Cpa at the edge of F-actin cap is affected. By inserting the GFP right after the Cpa gene with CRISPR, dynamics of Cpa can be observed. During the interphase, the distribution of Cpa clusters is affected, Cpa clusters are mainly localized to the intercap domain. Dia localizes to the downstream of Kinesin-1, which is also required for the distribution of Cpa clusters at the intercap region. Moreover, I found that Kinesin-1 and plus ends of microtubules are accumulated at the cap domain during the syncytial interphase. I also found that APC2 coprecipitates with Kinesin-1. Although the cellularization is affected in APC2 d40 truncation embryos, the localization of cortical components is comparable, different domains are clearly separated. The disruption of cellularization in APC2 d40 truncation embryos may due to reductions of Slam and Amphiphysin at the basal domain. 
I also mapped functions of the slam mRNA sequence for its localization and Slam protein translation. The results indicated that the entire slam mRNA sequence is required for robustly Slam protein expression. Apart from the slam mRNA sequence is essential for Slam protein expression, slam mRNA sequence is also required for the localization of slam mRNA. slam mRNA coding region from $507 \mathrm{nt}$ to $1576 \mathrm{nt}$ has an effect on slam mRNA localization and Slam protein expression. slam mRNA coding sequence from $2818 \mathrm{nt}$ to $3522 \mathrm{nt}$ is required for slam mRNA localization. 
Part A Cortical patterning in syncytial embryos: the link between microtubules and actin cortex 


\section{Introduction}

\subsection{Cortical polarization and functions of cytoskeleton in cell polarity}

Cortical polarization in cells includes spatial and temporal regulation of cortical components on the membrane, which leads to the asymmetric distribution of cortical proteins. The asymmetric distribution of cortical components induces cell polarity, which is essential for cell functions and morphological complexity during development. For example, stem cells utilize cell polarity to segregate morphological components asymmetrically and produce cell fate diversity for daughter cells. A conserved protein which has been shown to control stem cell fate is Scribble, which has been identified in Drosophila and mammals. In adult mouse stem cells, Scribble is asymmetrically and symmetrically distributed in dividing cells. The deletion of scribble affects the proliferation and the fate of muscle stem cells (Ono et al., 2015). For differentiated cells, cell polarity is needed for the formation of spatially restricted structures, such as cell-cell junctions (Sun and Stathopoulos, 2018). Furthermore, the cortical polarity is essential for the morphological complexity of embryos. In early embryonic development, the establishment of basic axes in embryos (e.g. anterior-posterior, dorsal-ventral) is required for organogenesis during the gastrulation stage (Hall, 1998). Loss of cell polarity in epithelial cells induces diseases, such as cancer. Alterations of apical-basal polarity in epithelial cells induce epithelial columnar shape defects and mesenchymal-like morphology, which are typical for invasive cancer cells (Royer and Lu, 2011; Woodham and Machesky, 2014).

Similar to the cell cortex, the cytoskeleton is polarized. The cytoskeleton is a structural component of cells, which consists of microtubules, F-actin filaments and intermediate filaments (Heng and Koh, 2010; Karr and Alberts, 1986). Filaments of cytoskeleton not only provide mechanical support, but are also essential for cortical polarization (Dogterom and Koenderink, 2019a). Furthermore, microtubules and Factin filaments generate tracks for motor proteins. For example, Kinesin-1 moves along microtubules from the minus end to the plus end, which is involved in direct transport (Ross et al., 2008). Also microtubules form mitotic spindles during mitosis, the bipolar mitotic spindle is essential for the segregation of chromosomes and cell 
division (Fraschini, 2017; Petry, 2016). Kinesin-5 binds and moves along antiparallel microtubules of mitotic spindle during mitosis, exerting force to push chromosomes separation during anaphase (Kapitein et al., 2005).

Moreover, centrosomes and its associated microtubules are required for cortical patterning, as they supply signals for cortical polarization (Acharya et al., 2014; Raff and Glover, 1989a). The former report indicated that centrosomes could initiate cortical polarization at any position. In C. elegans, centrosome may induce cortical polarization in three different ways, including direct contact, diffusible signals, and microtubule-dependent signals (Bienkowska and Cowan, 2012).

F-actin filaments as another element of cytoskeleton, also play an important role in cortical polarization. For example, Myosin II migrates along F-actin filament, promoting the epithelial apical contraction during ventral furrow formation (Coravos and Martin, 2016; Lv and Großhans, 2016). Moreover, Myosin superfamily proteins take part into the asymmetric distribution of cortical proteins (Cheeks et al., 2004; Munro et al., 2004).

\subsection{Microtubules and related proteins}

Microtubules are polar filaments, they have the plus end and the minus end. Microtubules are polymerized with $\alpha$-tubulin and $\beta$-tubulin heterodimers in the presence of GTP. The diameter of microtubules is $25 \mathrm{~nm}$ (Desai and Mitchison, 1997). Microtubules have multiple functions in cells, such as positioning of nuclei and organelles (Varshney et al., 2019; Xiang, 2018), cytoplasm organizing (Lu et al., 2016; Palacios et al., 2002). Furthermore, microtubule associated proteins like Kinesin-1 can regulate the cell polarity (League and Nam, 2011).

Microtubules are dynamic structures, with $\alpha \beta$-tubulin binding to and polymerizing at the plus end, disassemble and slowly assemble at the minus end of microtubules (Weingarten et al., 1975). Because of different nucleation centers, microtubules are divided into centrosomal microtubules and non-centrosomal microtubules. Centrosome, as a typical microtubule-organizing center (MTOC), is responsible for the nucleation and organization of centrosomal microtubules (Conduit et al., 2015; Sanchez et al., 2017; Wu et al., 2017). The polymerization of centrosomal microtubules starts with the nucleation and formation of the $y$-tubulin ring complex (Bouissou et al., 2014; Song et al., 2018; Thawani et al., 2018). The y-tubulin ring 
complex acts as a template for the microtubule polymerization. Non-centrosomal microtubules start their growth from the cortical loci, where Patronin and Short stop (Shot) form nucleation center without y-tubulin (Nashchekin et al., 2016; Sanchez et al., 2017).

Dynamics of microtubules are regulated by microtubule-associated proteins (MAPs). One of the MAPs is Tau (Weingarten et al., 1975), which not only enhances the polymerization of microtubules but also acts as a stabilizer for microtubules (Breuzard et al., 2013; Weingarten et al., 1975). Furthermore, Kinesin-13s bind and disassemble microtubules at spindle poles in Xenopus laevis (Aizawa et al., 1992; Hunter et al., 2003; Wordeman and Mitchison, 1995).

There are at least 20 different families of microtubule plus-end tracking proteins (+TIPs), including canonical end-binding (EB1) and CLIP170 (Perez et al., 1999; L.-K. Su et al., 1995, p. 1). EB1 is a relatively small protein, the molecular weight of EB1 protein is about $30 \mathrm{KDa}$. EB1 and its homologs are highly conserved, which are accumulated to the growing end of microtubules (Schuyler et al., 2001), regulating the stability and polymerization of microtubules. Also the former report indicated that EB1 is essential for the localization of CLIP170 at the plus end of microtubules. In vitro, CLIP170 bound to the plus end of microtubules in the presence of EB1. In the absence of EB1, CLIP170 moved diffusively along microtubules (Dixit et al., 2009).

Adenomatous polyposis coli (APC), as a tumor suppressor gene, is conserved from Drosophila to human beings. At the C-terminus of APC protein, EB1 binding region exists (Morrison, 2009). The interaction between EB1 and APC forms a bridge among microtubules, F-actin filaments and cell membrane, which is essential for the cell migration and mitosis (L. K. Su et al., 1995).

Motor proteins such as Kinesin proteins and Dynein proteins take advantage of the microtubule polarity, moving along microtubules to transport cargos such as organelles and protein complexes by consuming energy from ATP hydrolysis (Brendza et al., 2002; Hirokawa, 1998, 1982; Hirokawa et al., 2009).

Kinesin-1 is a (+)-end motor protein, the movement of Kinesin-1 depends on microtubules. As an important member of the Kinesin protein superfamily, Kinesin-1 consists of two light chains and two heavy chains. The light chain of Kinesin-1 is important for its activity regulation and cargo binding. The heavy chain of Kinesin-1 has microtubule-binding domain and ATP hydrolysis domain (Hirokawa et al., 2009, p. 1; Sanger et al., 2017), which are essential for Kinesin-1 movement. 
Kinesin-1 is required for multiple cellular processes, such as the mRNA localization (Gáspár et al., 2017), cargo transport (Pan et al., 2019; Schimert et al., 2019), and skeletal muscle nuclear positioning (Metzger et al., 2012; Pilling et al., 2006). In early embryonic development of Drosophila embryos, Kinesin-1 is required for the membrane ingression and the nuclear elongation during cellularization (Winkler et al., 2015).

Furthermore, Kinesin-1 is required for the patterning of embryonic axes. For example, Crumbs, as a transmembrane protein, is conserved from $C$. elegans to human beings (Médina et al., 2002). Crumbs was first identified in Drosophila embryos, it localizes to the apical domain during cellularization, and forms a complex with Patj and Stardust (Horne-Badovinac et al., 2008; Tepass et al., 1990). Crumbs has also been found in photoreceptor cell morphogenesis, Crumbs can control the position and integrity of photoreceptors (Izaddoost et al., 2002; Pellikka et al., 2002). Kinesin-1 is required for the localization of Crumbs in Drosophila photoreceptors. For example, the previous publication reported that when the expression level of Kinesin-1 was reduced in Drosophila pupa, Crumbs not only mislocalized to the distal section but also lost accumulation at the proximal section. The mislocalization of Crumbs enhanced the rough eye phenotype in Drosophila (League et al., 2011).

Drosophila oocyte is the place for the abdomen and germ cell formation. The anterior and posterior of Drosophila oocyte are defined with oskar mRNA and bicoid mRNA respectively (Kim-Ha et al., 1991; Simpson-Brose et al., 1994). The posterior localization of oskar mRNA in oocyte depends on two different mechanisms: (1) oskar mRNA is transported by Kinesin-1, which moves along microtubules to the posterior of oocyte (Brendza et al., 2000; Nieuwburg et al., 2017) and (2) the translocalization of oskar mRNA to the posterior depends on ooplasmic streaming, which is driven by Kinesin-1 (Lu et al., 2016; Palacios et al., 2002).

For ooplasmic stream-dependent oskar mRNA localization, microtubule-microtubule sliding is required. Kinesin-1 slides free cytoplasmic microtubules along the actin cortex anchoring microtubules, which contributes to the ooplasmic streaming. The heavy chain, but not the light chain, of Kinesin-1 is essential for microtubule sliding, as microtubule sliding was significantly reduced in Kinesin-1 heavy chain mutant embryos. In Kinesin-1 heavy chain mutant embryos, the localization of oskar mRNA is affected, the localization of oskar mRNA at the posterior of the oocyte is not as restricted as in wild type. However, the anterior localization of bicoid mRNA in the 
oocyte is not affected in Kinesin-1 mutant embryos. (Lu et al., 2016).

For the non-centrosomal microtubule-dependent oskar mRNA localization, the stability of the microtubule plus end is important. As Dynactin can extend plus end of microtubules, it is important for the localization of oskar mRNA at the posterior part of oocyte. The localization of Dynactin also depends on Kinesin-1 (Nieuwburg et al., 2017).

The localization of bicoid mRNA to the anterior part of oocyte depends on Dynein (Kugler et al., 2009; Trovisco et al., 2016). Dynein is a (-)-end motor protein, by consuming ATP, cytoplasmic Dynein migrates along microtubules to the minus end, responsible for the majority of microtubules minus-end motilities, such as organelles and vesicles transport (He et al., 2005; Rao et al., 2017; Wagner et al., 2004).

\subsection{Actin filaments and related proteins}

Actin filaments are polar structures, consist of pointed (minus) ends and barbed (plus) ends. As a main component of the cell cortex, actin filaments are essential for multiple cellular processes, including wound healing (Bement et al., 1993; Benink et al., 2005), cell migration (Callan-Jones et al., 2016), and cellular protrusion (Démoulin et al., 2014). These processes depend on the force generated by the elongation of actin filaments and the Myosin II movement driven by ATP hydrolysis.

Actin filaments are polymerized with G-actin monomers. The elongation of actin filaments starts with actin nucleation. Arp2/3 complex, Formins, and Spire are required for nucleation (Dominguez et al., 2011; Pring et al., 2003; Rottner et al., 2010). For example, the depletion of Arp $2 / 3$ complex affects actin nucleation, which induces cortical instability (Loria et al., 2012). After nucleation, actin filaments polymerize with Profilin-bound G-actin monomers in the presence of ATP (Courtemanche et al., 2013; Nejedla et al., 2016; Suarez et al., 2015). Profilin takes part in actin nucleation and actin filament elongation in eukaryotic cells. The ratio between Profilin and G-actin is important for the assembly of actin network. In wild type cells, the ratio between Profilin and G-actin is about 0.8 . When the ratio is higher than normal, the Formin contractile ring formation is improved. When the ratio is lower than 0.8, it tends to form Arp2/3 dependent patches (Burke et al., 2014).

Although the actin filament elongation happens in both pointed and barbed ends, elongation velocity at the barbed end is faster than at the pointed end. In vitro, actin 
monomers polymerize spontaneously in the presence of $\mathrm{Ca}^{2+}$ or $\mathrm{Mg}^{2+}$ (Kabsch et al., 1990), and polymerization dynamics depend on the concentrations of the actin monomers and Profilin (Courtemanche et al., 2013).
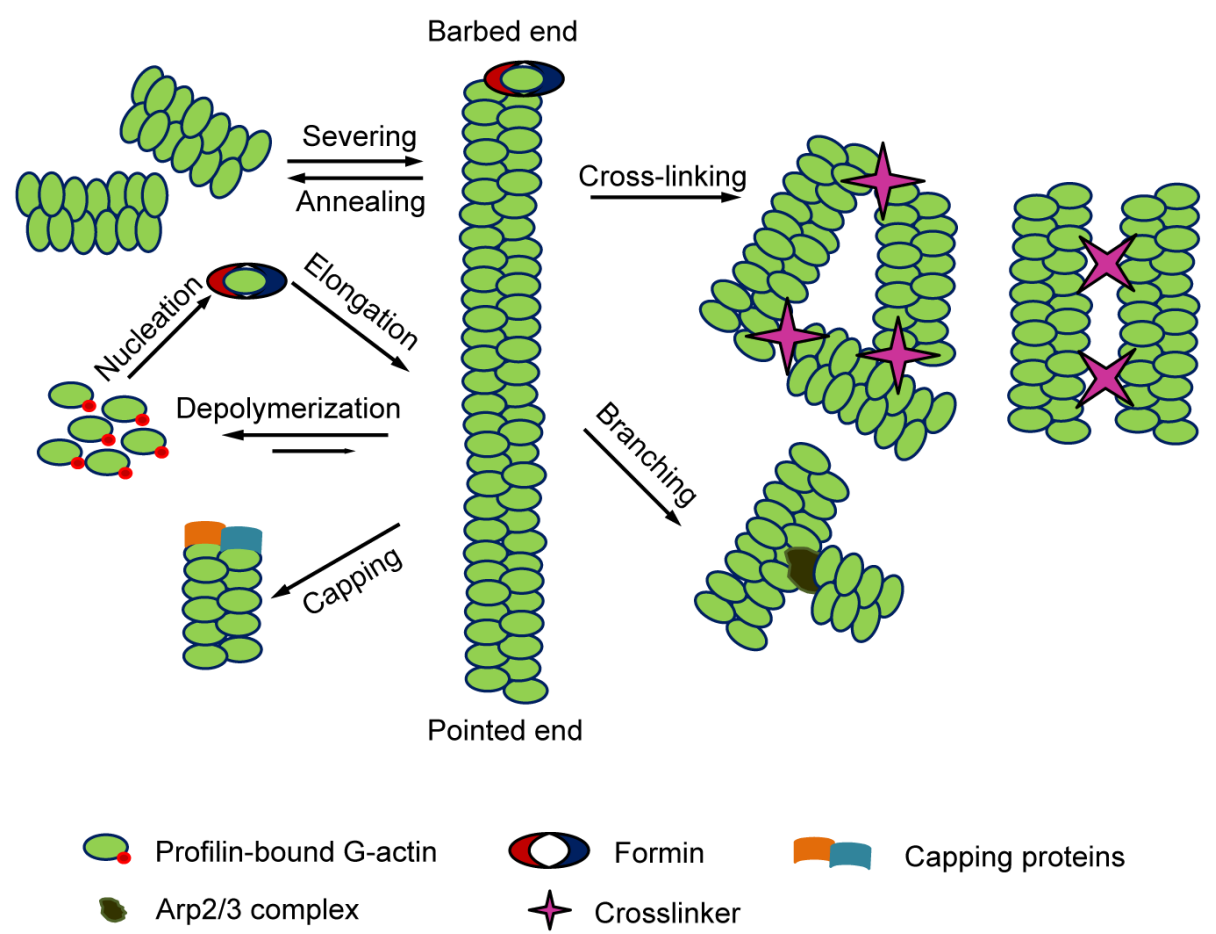

Figure 1 Scheme of actin dynamics and actin-binding proteins in non-muscle cells.

The actin filaments are highly dynamic structures, including severing and annealing, nucleation and elongation, depolymerization, capping, cross-linking and branching. The severing of actin filaments depends on Cofilin, which binds to the side of the actin filaments. Actin-binding proteins that regulate actin polymerization include Capping proteins, Formins, cross-linking proteins, and branching protein Arp2/3 complex. Proteins that are required for nucleation include Arp2/3 complex, Formins, and Spire. The elongation of actin filaments starts with actin nucleation. Both Formins and VASP bind to the plus end of actin filaments and promote actin elongation. Formins and VASP also can inhibit actin filament capping. The capping protein of F-actin filaments includes Capping $\alpha$ and Capping $\beta$. Capping proteins bind to the plus end of actin filaments, which inhibit the polymerization of actin filaments. The Arp2/3 complex can bind to the side of actin filaments and start daughter filaments. This schematic is adapted from Pollard, 2016.

Dynamics of actin assembly such as actin filament branching, capping, and severing depend on multiple actin-binding proteins. For example, the Arp2/3 complex is not only essential for the actin nucleation but also for the actin filaments branching. Arp2/3 binds to the side of the mother actin filament, establishing the base for the branch growth of actin filaments (Rouiller et al., 2008). Capping proteins including Capping $\alpha$ and Capping $\beta$, are major capper proteins in non-muscle cells. Capping proteins localize to the barbed end of actin filaments, regulating the stability and polymerization of actin filaments (Cooper and Pollard, 1985; Edwards et al., 2014a). Capping proteins bind to the plus end of actin filaments with high affinity. The activity 
of Capping proteins can be regulated by Myotrophin (Zwolak et al., 2010) and Formins. Formins are required for the polymerization of actin filaments. One of the Formin proteins is Diaphanous (Dia). Dia consists of $\mathrm{FH}$ domains, it binds to and promotes the elongation of actin filaments at the barbed end (Higashida et al., 2004; Kovar and Pollard, 2004; Paul and Pollard, 2009).

Myosin superfamily proteins are actin-related motor proteins, different members of Myosin superfamily have a vast structural and functional diversity. In muscle cells, non-muscle Myosin II heavy chain filaments and actin filaments form sarcomeres, which are basic units of muscle. Non-muscle Myosin II filaments migrate along antiparallel actin filaments, inducing muscle contraction (Craig and Woodhead, 2006; Squire, 1972).

Myosin proteins not only induce the contraction in muscle cells but are also required for a set of cellular processes. One of the most important member in Myosin superfamily is Myosin II. Myosin II consists of two heavy chains, two regulatory light chains, and two essential light chains. For example, Myosin II is essential for blebs contraction in mammalian cell cytokinesis (Babkoff et al., 2019; Taneja and Burnette, 2019). For mammalian cells, there exists three isoforms of Myosin II: Myosin IIA, Myosin IIB and Myosin IIC, they have distinct biophysical properties. When Myosin IIA is depleted in HeLa cells, the bleb contraction failed. While Myosin IIB and Myosin IIC are not sufficient for the membrane contraction during cytokinesis (Taneja and Burnette, 2019). The contractile rings in cytokinesis consist of actin, Myosin II and other components. The contraction of contractile rings is also regulated by Rho kinase, Rho kinase phosphorylates the regulatory light chain of Myosin II. Rho kinase also can regulate Dia activity to promote the actin polymerization (Babkoff et al., 2019; Watanabe et al., 2008).

The localization of Myosin II is vital for ventral furrow formation. The Drosophila ventral furrow formation starts during gastrulation, which is an important morphogenetic process, the ventral furrow formation induces the internalization of mesodermal precursors (Krueger et al., 2018; Martin et al., 2009). During the gastrulation, different accumulations of Myosin II between apical and basal domains are important. The previous report showed that when the ventral furrow formation started, Myosin II accumulated to the apical domain, whereas the amount of Myosin II at the basal surface decreased (Krueger et al., 2018). When the amount of Myosin II at the basal domain was increased by the RhoGEF2-CRY2/CIBN optogenetic system, 
cells at the activated region did not internalize within $10 \mathrm{~min}$. Also, the basal accumulation of Myosin II during the ventral furrow formation induced a lack of invagination as well as cell shape was changed, because of the apical constriction of the cell was inhibited. All these changes induced the failure of ventral furrow formation (Krueger et al., 2018).

Furthermore, Myosin II can organize F-actin filaments (Wollrab et al., 2019). V. Wollrab et al. showed that, in vitro, without ATP and Myosin II, actin filaments were randomly distributed. When Myosin II was added, the organization of actin filaments immediately changed and actin asters formed within 36s. They also found that Myosin II tracked along F-actin filaments with a typical mean speed of $2 \mu \mathrm{m} / \mathrm{s}$. When Myosin II moved to the plus end of F-actin filament, it attached to the filament and moved along another filament, joining plus ends of both F-actin filaments together. Furthermore, they found that Myosin II mediated merging and splitting of F-actin filament asters in vitro.

\subsection{The crosstalk between microtubules and F-actin filaments}

Functions of microtubules and F-actin filaments in cell biology and biophysics have been studied for several decades and interactions between microtubules and F-actin filaments in core processes have been concerned. The direct crosstalk between microtubules and F-actin filaments can mediate polymerization between each other. In mammalian intestinal epithelial cells, multi-domain crosslinking protein ACF-7, binds to both F-actin filaments and microtubules and induces the polymerization of microtubules along with actin bundles (Nashchekin et al., 2016; Preciado López et al., 2014).

In Drosophila oocyte, Patronin and Short stop (Shot) form a bridge between the minus end of microtubules and the actin cortex (Goodwin and Vale, 2010; Khanal et al., 2016). In Patronin and Shot double mutant embryos, the organization of microtubules was affected and the localization of Cad99c at the apical membrane was compromised as well. Meanwhile, the mis-organization of microtubules induced the accumulation of Rab11 at the cytoplasm. Rab11 is important for the recycling endosome and the membrane components localization (Khanal et al., 2016).

Besides, microtubules promote F-actin polymerization by recruiting CLIP-170 and 


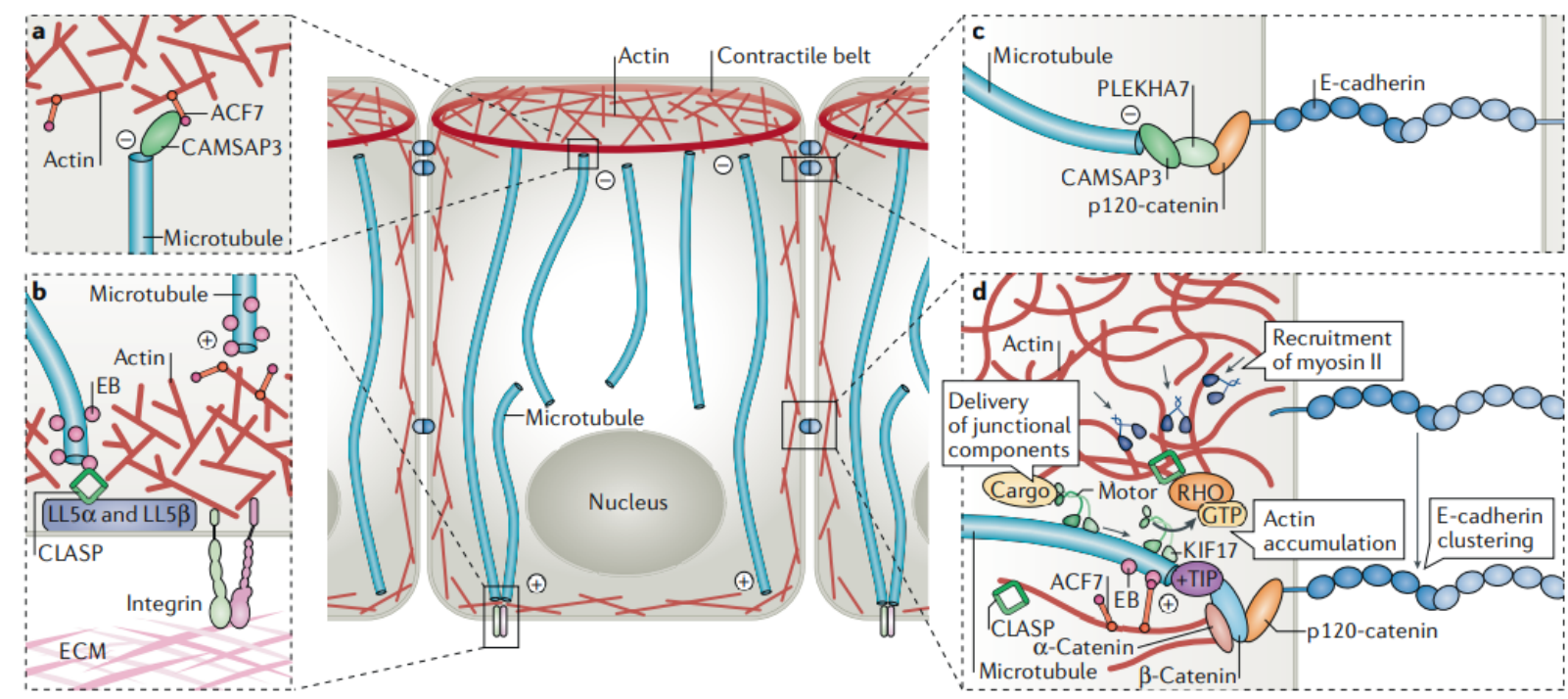

Figure 2 Microtubules and actin filaments crosslink in epithelial cells.

Microtubules interact with actin filaments in different ways. (a) The minus end of microtubules connects to the actin cortex via CAMSAP3 protein and spectraplakin ACF-7 in mammalian intestinal epithelial cells. In Drosophila, the linker between the minus end of microtubules and actin cortex is the Patronin/Shot complex. Patronin also acts as nucleation center for non-centrosomal microtubules. (b) LL5 $\alpha$ and LL5 $\beta$ are microtubule plus end binding proteins, they bind with EB1/CLIP associating protein (CLASP), which form the connection between the plus end of microtubules and the cell cortex (Hotta et al., 2010). (c) The minus end of microtubules connects to cadherin-based adherens junction via CAMSAP3, PLEKHA7, and p-120 catenin (Meng et al., 2008). (d) EB1, $\beta$-catenin and p-120 catenin form bridge between the plus end of microtubules and cortical actin. The polymerization of microtubules influences the actin filaments polymerization, which might contribute to the adherens junction formation (Shahbazi et al., 2013; Stehbens et al., 2006). Modified from M. Dogterom \& G.H. Koenderink, 2019.

mDia1 to the plus end of microtubules to stimulate actin nucleation (Henty-Ridilla et al., 2016). In addition to promoting polymerization between each other, F-actin filaments offer positions for microtubules anchoring at the cortex. Moreover, F-actin filaments form a barrier at the cell cortex to inhibit the growth of microtubules, the actin barrier also induces catastrophes of microtubules (Janson et al., 2003).

Furthermore, the polarity of epithelial cells is maintained via interactions between microtubules and the actin cortex, with both plus and minus ends of microtubules binding to the actin cortex (Nashchekin et al., 2016; Noordstra et al., 2016; Toya et al., 2016).

\subsection{Cortical polarity in epithelial cells and C. elegans embryos}

Mechanisms for cortical polarization have already been investigated in different model organisms. Among different model organisms, cortical polarization is well 
understood in epithelial cells. The cortical polarization of epithelial cells generates apical-basal polarity axis, the cell cortex divides into the apical domain and basolateral domain, which are segregated by the apical junction complex. Partitioning-3 (Par-3), Par-6 and atypical protein kinase C (aPKC) form Par complex and localize to the apical domain. Lethal giant larvae ( $\mathrm{Lgl})$, Scribble and Disc large (Dlg) form Scribble complex and localize to the basolateral domain.

The activity and localization of these protein complexes can be regulated by each other (Hutterer et al., 2004; Plant et al., 2003; Yamanaka et al., 2006). On the one hand, phosphorylation of $\mathrm{Lgl}$ at the apical domain by aPKC induces the $\mathrm{Lgl}$ inactivation, which excludes Scribble complex from the apical domain. On the other hand, the basolateral localization of the Scribble complex restricts the Par complex at the apical domain. Par- 6 is essential for the establishment of epithelial cell polarity, as it is required for the localization of aPKC and Par-3 at the cell cortex. Lgl prevents basolateral localization of Par-6. In Lgl mutant embryos, the restriction of Par-6 protein at the apical domain is lost, Par-6 localizes to the basolateral domain as well (Hutterer et al., 2004).

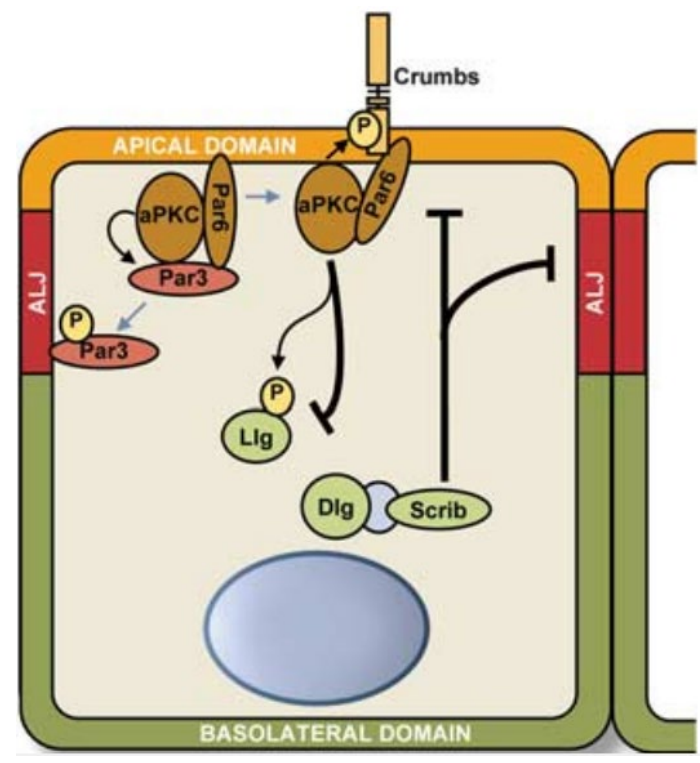

Figure 3 Asymmetric distribution of cortical components in epithelial cells.

The cortex of epithelial cells is polarized into the apical domain (yellow) and basolateral domain (green). Different cortical components localize to different domains. Par complex (Par-3, Par-6, and aPKC) and Crumbs complex (Crumbs, Pals1, Patj and Lin-7) localize to the apical domain, Scribble complex ( $\mathrm{Lgl}$, Dlg and Scribble) localizes to the basolateral domain. aPKC phosphorylates and inactivates $\mathrm{Lgl}$, excluding $\mathrm{Lgl}$ from the apical domain. While aPKC phosphorylates Crumbs and promotes localization of Crumbs to the apical domain. Lgl excludes Par complex and Crumbs complex from the basolateral domain. Modified from C.Royer \& X. Lu, 2011.

Cortical polarization not only exists in epithelial cells, but also can be found in early 
embryonic development of $C$. elegans embryos. Cell polarity already exists at onecell stage of $C$. elegans embryos. Par proteins, which were first identified in $C$. elegans embryos, are required for the cell polarization. Par proteins are conserved from worms to mammals. Par proteins are important as they take part into multiple developmental processes. In C. elegans embryos, Par proteins, including six Par protein members, are asymmetrically distributed at one-cell stage. For example, Par3 and Par-6 localize to the anterior part of $C$. elegans embryos, Par-1 and Par-2 accumulate to the posterior part (Cheeks et al., 2004; Cuenca et al., 2003; Munro et al., 2004).

The asymmetric distribution of Par-3 and Par-6 proteins in C. elegans zygotes depends on the contraction of actomyosin. Before Par proteins asymmetrically distribute to the anterior and posterior domains, Par-3 and Par-6 localize throughout the embryo cortex. Par-1 and Par-2 can be found in the cytoplasm. During the cortical polarization, actomyosin contraction generates a cortical flow towards the anterior part of $C$. elegans embryos, inducing the anterior translocation of Par-3 and Par-6. Meanwhile, Par-1 and Par-2 proteins migrate from the cytoplasm of embryos to the posterior part. The asymmetric distribution of Par proteins is affected when the level of cortical actomyosin is reduced (Kumfer et al., 2010; Piekny and Mains, 2002). For instance, the previous publication showed that Rho-1 regulated actomyosin activity by phosphorylating Myosin regulatory light chain. When Rho- 1 was depleted with RNAi, the asymmetric distribution of Par proteins was influenced (Plant et al., 2003).
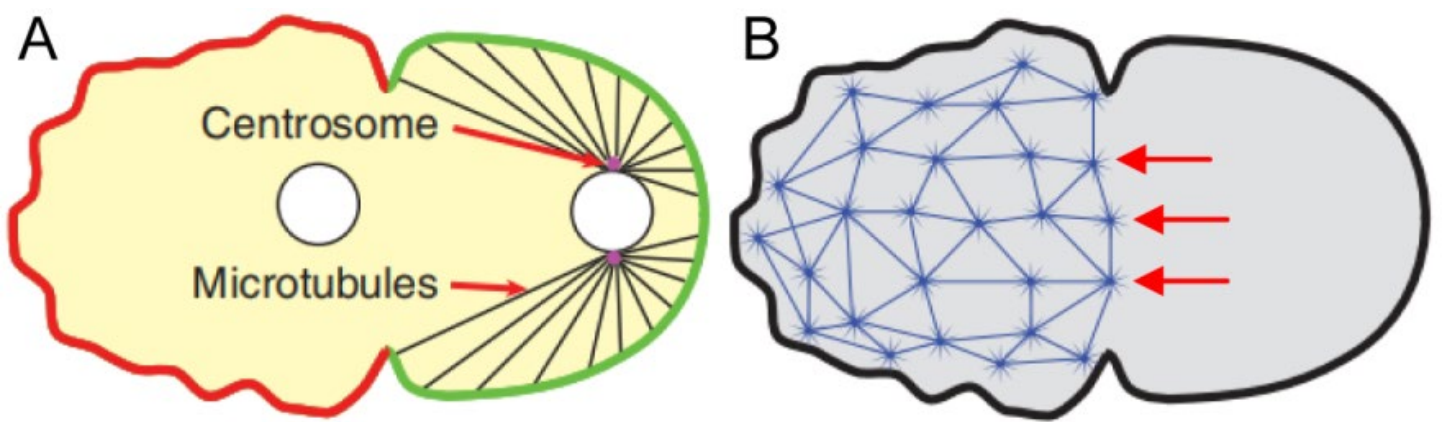

Figure 4 Translocation of Par-3 to anterior by the actomyosin contraction.

(A) Asymmetric distribution of Par-3 (red) and Par-2 (green) in C. elegans zygote. The anterior distribution of Par-3 is due to cytoplasmic flow caused by the actomyosin contraction. At the same time, Par-2 migrates from cytoplasm to the membrane along microtubules. The centrosome is nucleation center and minus end of microtubules. (B) Anterior contraction of Myosin filaments (blue). The red arrows indicate the direction of Myosin contraction. Modified from J. Nance \& J.A. Zallen, 2011. 
The initiation and establishment of cortical polarity in C. elegans zygote relies on centrosomes (Cowan and Hyman, 2004). The report showed that when centrosomes were ablated with UV laser before polarization initiated in C. elegans zygote, the posterior accumulation of Par-2 was lost and Par-2 was evenly distributed on the embryo cortex. When centrosomes were ablated with UV laser after polarization started, the localization of Par-2 was not affected, Par-2 accumulated at the posterior of $C$. elegans zygote.

\subsection{The formation of different cortical domains and the polarity regulation during Drosophila early embryonic development}

The Drosophila embryos is a good model for investigating the function of proteins in early embryonic development and cortical polarization, as cortical polarization is one of the main events in Drosophila early embryonic development.

The polarity of embryo cortex is important for the embryonic differentiation and development. In Drosophila embryos, during the pre-blastoderm stage (0-9 nuclear cycles), nuclei exist in the york of Drosophila embryos and membrane components are evenly distributed on the embryo cortex. There is no cortical domain differentiation, Myosin II and F-actin are uniformly distributed on the cortex (Karr and Alberts, 1986; Warn et al., 1984). At this stage, components on the embryo cortex are highly mobile. For example, when growth-associated protein 43 (GAP43) and Toll were photobleached during the pre-blastoderm stage, the fluorescent intensities of GAP43 and Toll at photobleached regions were almost recovered in 60s (Mavrakis et al., 2009). This is due to the diffusion of GAP43 and Toll from near regions.

Nuclei reach to the embryo cortex in nuclear cycle 10. In syncytial blastoderms (from 10-13 nuclear cycles), the membrane polarization and the asymmetric distribution of cortical components start. With the asymmetric distribution of membrane components, the cortex of Drosophila embryos divides into cap domain and intercap domain during the interphase. Cortical components are asymmetrically distributed on the embryo cortex. F-actin, Canoe, ELMO/Sponge complex, and Moesin can be found the cap domain (Karr and Alberts, 1986; Rikhy et al., 2015; Schmidt et al., 2018). The unconventional GEF complex ELMO/Sponge is required for the F-actin arrangement at the cap domain. In ELMO mutant embryos, the F-actin cap cannot form during the 
interphase of syncytial stage (Schmidt et al., 2018). Proteins such as Slam, Myosin II, and E-Cadherin can be found at the intercap domain (Royou et al., 2003; Schmidt et al., 2018; Warn et al., 1980). The activity of Myosin II at the intercap domain is regulated by the Rho signaling pathway. With cap and intercap domains formation, the mobility of cortical components decreases. FRAP experiment showed that components on the same cap domain are easily migrate to the bleached area, while components exchange between neighboring domains declines compares to preblastoderm stage (Mavrakis et al., 2009).

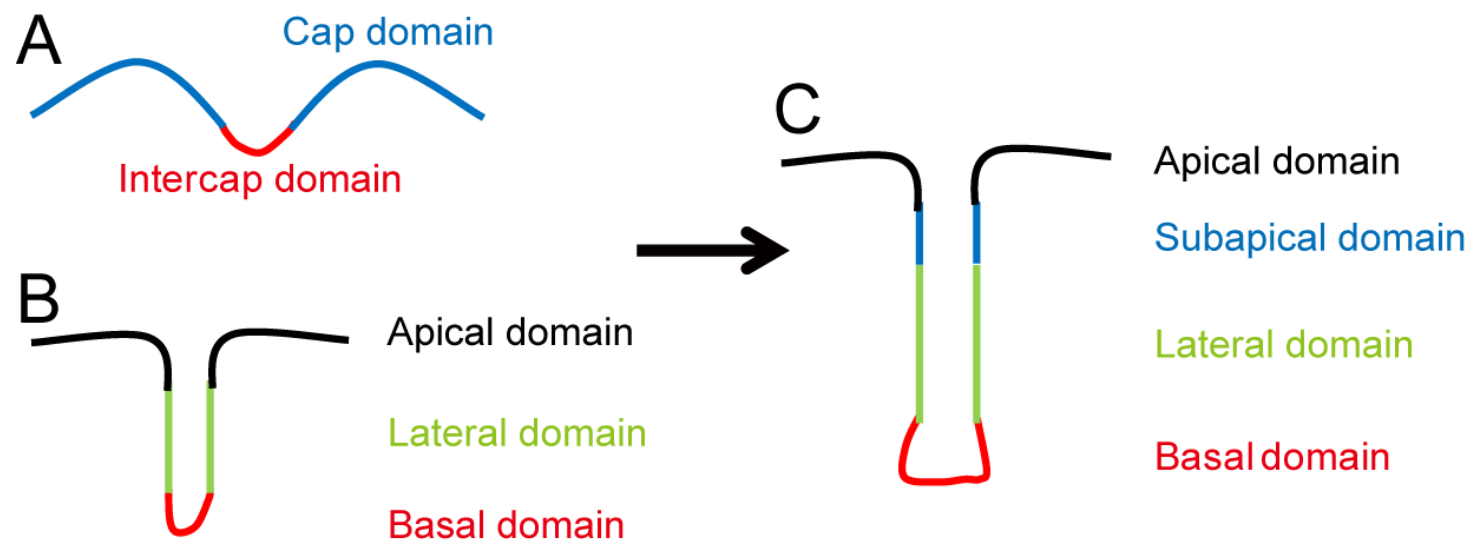

Figure 5 Dynamics of cortical domain in Drosophila early embryonic development.

Drosophila embryos show significant cortical polarity and asymmetric distribution of cortical proteins during early embryonic development. (A) Cortical domains during the syncytial interphase. From nuclear cycle 10 to 13 , nuclei migrate to the embryo cortex, cortical membrane differentiates into cap domain and intercap domain during the interphase. (B) Cortical domains during the mitosis of syncytial stage. During mitosis, with the membrane invagination and elongation, the metaphase furrow forms. The embryo cortex divides into apical domain, lateral domain, and basal domain. The metaphase furrow constricts at the end of mitosis. (C) Cortical domains during the cellularization stage. During the interphase of cell cycle 14, a new domain, subapical domain arises. The embryo cortex divides into apical, subapical, lateral, and basal domains. Cortical proteins are asymmetrically distributed at different domains. At the end of cellularization, nuclei are separated by cell membrane, embryos divide into about 6000 cells.

In syncytial blastoderms, during the mitosis, with the metaphase furrow formation, the embryo cortex separates into the apical domain, lateral domain, and basal domain. Canoe and ELMO/Sponge complex localize to the apical domain (Schmidt et al., 2018). Dlg localizes to the lateral domain (Harris and Peifer, 2004). Slam and Amphiphysin localize to the basal domain (Schmidt et al., 2018; Sokac and Wieschaus, 2008).

Cellularization starts during the interphase of cell cycle 14 in Drosophila embryos. During cellularization, a new domain arises: the subapical domain. The cortex of Drosophila embryos divides into four domains: apical, subapical, lateral and basal 
domains. Par-6 and Cdc-42 localize to the apical domain (Hutterer et al., 2004). The subapical domain exists between the apical domain and lateral domain, where Canoe and ELMO/Sponge complex localize (Schmidt et al., 2018). Subapical domain comes up slightly later than lateral domain and basal domain, the clear segregation of subapical domain and basal domain can be found at the onset of cellularization.

The formation of cortical domain and the localization of proteins are accurately regulated during cellularization. The ELMO/Sponge complex localizes to the upstream of Canoe, the subapical localization of Canoe is control by ELMO/Sponge complex. In the ELMO mutant embryos, during cellularization, the subapical domain localization of Canoe is affected. Canoe localizes to the subapical domain directs the position of Bazooka and adherens junction (Bonello et al., 2018; Choi et al., 2013; Schmidt et al., 2018).

Proteins such as Dlg, Scribbled and E-Cadherin localize to the lateral domain (D. Bilder and Perrimon, 2000; Thomas and Williams, 1999). F-actin, Patj, and Peanut localize to the basal domain. The localization of F-actin affects the distribution of Patj and Peanut. For example, during cellularization, when Drosophila embryos were injected with Latrunculin A (F-actin polymerization inhibitor), Patj and Peanut were mislocalized at the basal domain (Mavrakis et al., 2009).

Slam also can be detected at the basal domain, which is essential for the furrow ingression. The localization of Slam at the basal domain is regulated by Nuf (nuclear fallout). Nuf is required for the function of recycling endosome (Acharya et al., 2014; Lecuit et al., 2002). Dynamics of Slam are different during different stages of cellularization. For instance, the previous report showed that, at the onset of cellularization, the GFP-Slam fluorescence recovered rapidly and fully in about $7 \mathrm{~min}$ after photo-bleaching. However, in the middle of cellularization, the recovery speed of GFP-Slam fluorescence decreased and the GFP-Slam fluorescent intensity was less than half compared to the unbleached region after 10 min (Acharya et al., 2014).

During cellularization, not only the protein localization but also the amount of cortical components is important for cortical polarization. For example, the amount of Slam at the basal domain is crucial for cellularization. The amount of Slam at the basal domain increases rapidly during cellularization (Yan et al., 2017; Yan and Großhans, 2018). At the onset of cellularization, when Drosophila embryos transit from cell cycle 13 to cell cycle14, the amount of Slam protein at the basal domain increases about 6 fold. Following with this, the amount of slam RNA increases as well. 
The Slam accumulation at the basal domain is essential for Myosin II, RhoGEF2 localization. The previous study showed that in slam mutant embryos, the amount of Myosin II at the basal domain was slightly decreased, and the localization of Myosin II was also affected (Lecuit et al., 2002). Rho signaling and RhoGEF2 are essential for furrow formation and F-actin localization, they also regulate the activity of Myosin II. The localization of RhoGEF2 at the basal domain is lost in Slam depleted embryos (Großhans et al., 2005; Wenzl et al., 2010).

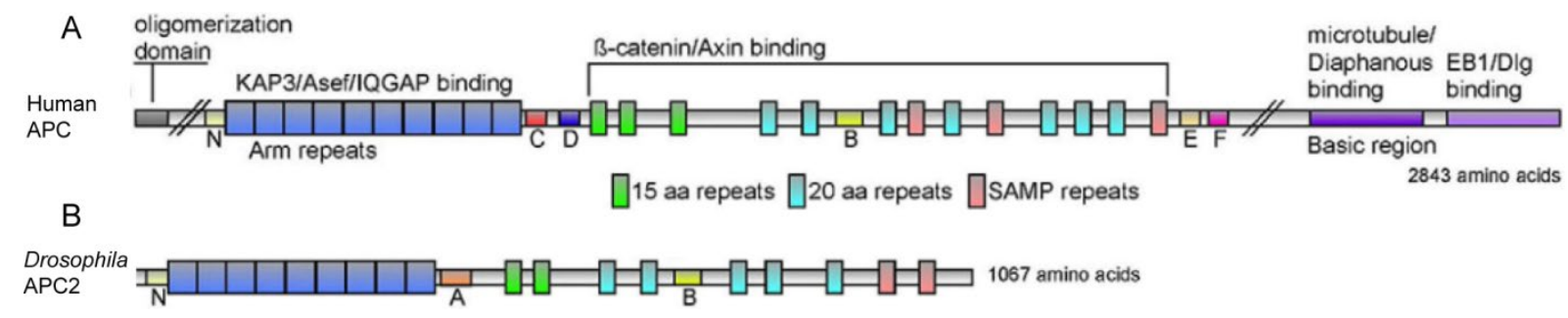

Figure 6 Schematic of human APC and Drosophila APC2.

(A) Multiple domains of the human APC protein. The human APC protein is made up of 2843 amino acids, APC protein contains different functional domains. Armadillo repeats exist at the C-terminal of APC protein. Proteins that bind to this domain include the Kinesin protein linker KAP3 (Jimbo et al., 2002), Rac-GEF protein Asef (Kawasaki et al., 2000). $\beta$-catenin and Axin binding domains exist in the middle of APC protein, they are involved in the Wnt signaling pathway. The 15 amino acids repeat and the 20 amino acids repeat can bind to $\beta$-catenin. The 20 amino acids repeat is regulated by phosphorylation. The SAMP repeats can bind to Axin. Axin is required for the formation of the $\beta$ catenin destruction complex ( $\mathrm{Ji}$ et al., 2019). At the C-terminal of APC, there are Dia binding domain and EB1 binding domain. (B) Multiple domains of Drosophila APC2 protein. APC2 consists of 2843 amino acids. The Armadillo repeats and $\beta$-catenin and Axin binding domains exist at the $\mathrm{N}$-terminal and the middle of APC2 respectively. However, at the C-terminal of APC2, there is no Dia binding domain and EB1 binding domain (Webb et al., 2009, p. 2). Modified from Webb et al., 2009.

Adenomatous polyposis coli (APC) proteins are conserved from Drosophila to human beings. In Drosophila embryos, as a member of APC proteins, APC2 is required for cortical polarization during cellularizaiton. APC2 is important for the F-actin localization in Drosophila syncytial blastoderms. F-actin localization is affected at the metaphase furrow in APC2 truncation embryos, which induces the defects of cortical polarization (Webb et al., 2009). Different APC proteins in different species share same domains like Armadillo repeats domain and $\beta$-catenin binding domain. Since APC proteins have $\beta$-catenin binding domain, it is essential for the $\mathrm{Wnt} / \beta$-catenin signaling pathway, APC proteins can catalyze the phosphorylation of $\beta$-catenin (Guger and Gumbiner, 2000; Staal et al., 2002). APC proteins act as tumor suppressors, inhibit epithelial cells migration and regulate the polarity of epithelial cells. APC mutations have been found in a majority of colorectal cancers (Giles et al., 2003; Polakis, 2000). Moreover, APC proteins play a role in cellular adhesion. $\beta$ - 
catenin binding domain in APC proteins links to the transmembrane protein ECadherin (Su et al., 1993). In addition, APC proteins take part in the Wnt signaling pathway.

Diaphanous (Dia), as a member of Formin family proteins, binds to the plus end of Factin filaments. Dia has been reported to have multiple functions (Bogdan et al., 2014). For example, Dia was originally found essential for cytokinesis (Castrillon and Wasserman, 1994). Moreover, Dia is required for the F-actin filament nucleation and elongation. Dia binds to the barbed end of actin filaments to promote the elongation of actin filaments. The elongation of actin filaments is required for the metaphase furrow formation (Cao 2008, Webb, 2009), cell motility, and cellular protrusion (Velle and Fritz-Laylin, 2019; Zeng et al., 2019). In addition, Dia is needed for the cortical polarization and asymmetric distribution of cortical proteins in Drosophila embryos during cellularization (Yan et al., 2013). It has been shown that the lateral domain marker Dlg mislocalized in dia mutant embryos during cellularization. Dlg could be detected not only at the lateral domain but also at the basal domain in dia mutant embryos. Meanwhile, the junctional marker protein Armadillo had an overlap with Slam in dia mutants.

\subsection{Aim of the study}

The previous report indicated that Kinesin-1 is required not only for the fluctuation of centrosomes but also for the cellularization of Drosophila embryos. The invagination of cortical membrane in Drosophila embryos was severely compromised, the front of membrane stuck at the surface of cortex (Winkler et al., 2015). However, the mechanism about how Kinesin-1 affects the cellularization and the cytoskeletion in Drosophila embryos are not clear.

Here, to better understand how Kinesin-1 affects Drosophila early embryonic development, I investigated mechanisms of Kinesin-1 in cortical patterning during Drosophila early embryonic development. I first checked the localization of different cortical components and dynamics of GFP-Slam in wild type and Kinesin-1 RNAi embryos. Then as Kinesin-1 is a microtubule-dependent protein, cytoskeleton includes microtubules and F-actin filaments are crucial for cell polarization, I checked microtubules and F-actin filaments associated proteins in Kinesin-1 RNAi embryos. 


\section{Results}

\subsection{Cortical localization of Kinesin-1 in Drosophila embryos}

Kinesin-1 is a (+)-end motor protein, it moves along microtubule from the minus end to the plus end. Kinesin-1 composes of two Kinesin light chains (Klc) and two Kinesin heavy chains (Khc). Khc contains motor domain with ATPase activity, which is essential for Kinesin-1 binding and sliding on microtubules. Klc has functions in Kinesin-1 activity regulation and recognition of cargos (Sanger et al., 2017; Yang et al., 1988). Kinesin-1 is required for multiple cellular processes, such as mRNA localization (Gáspár et al., 2017), cargo transport (Brendza et al., 2002), patterning of embryonic axes and nuclear positioning in skeletal muscle (Brendza et al., 2002; Metzger et al., 2012). In Drosophila syncytial blastoderms, although Kinesin-1 is not essential for mitosis (Gallaud et al., 2014), centrosomes fluctuation is affected in Kinesin-1 depleted syncytial embryos (Winkler et al., 2015). Furthermore, Kinesin-1 is required for the membrane ingression during cellularization (Winkler et al., 2015). To better understand the function of Kinesin-1 during Drosophila early embryonic development, I analyzed the distribution of Kinesin-1 in Drosophila embryos with GFP-tagged Kinesin-1, also the Drosophila strain with Kate knock-in allele of Kinesin1 was used to check the distribution of Kinesin-1.

3.1.1 Kinesin-1 localizes to the cortex of Drosophila embryos during syncytial and cellularization stages

To get a full understanding of functions of Kinesin-1 for the cortical differentiation and cortical components distribution, I checked the distribution of Kinesin-1 in early embryonic development. Previous publications reported that Slam localizes to the tip of invaginating furrows and serves as a marker for the basal domain during mitosis and cellularization (Lecuit et al., 2002; Acharya et al., 2014; Yan et al., 2017). Khc is the essential element for Kinesin-1 protein. I imaged Drosophila embryos expressing Khc-GFP and Slam-mCherry with a z-step size of $1 \mu \mathrm{m}$ at the indicated time points. The interphase of cell cycle 13 before the mitosis started was defined as the time point $0 \mathrm{~min}$. The result indicated that Khc-GFP signal was observed at the embryo 
cortex (0-2 $\mu \mathrm{m})$ during interphase (0 $\mathrm{min}$ and $13 \mathrm{~min}$ ) and mitosis (6 min) (Figure 7). The syncytial stage and the cellularization stage of embryos could be distinguished from size and density of nuclei, nuclei were the places that Khc-GFP fluorescence excluded.

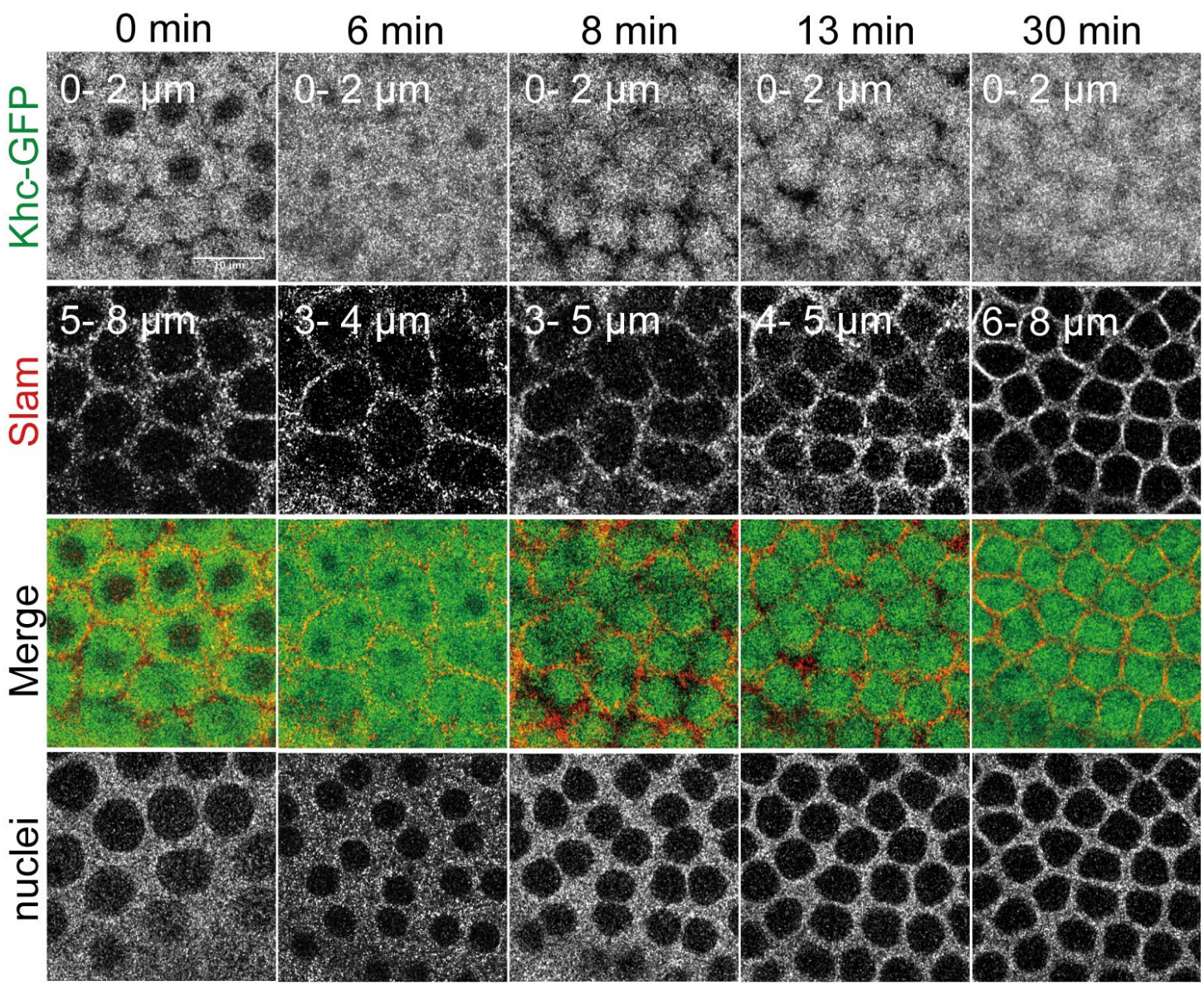

Figure 7 Kinesin-1 localizes to the apical domain during syncytial and cellularization stages.

Images from time-lapse recordings of Khc-GFP (green) and Slam-mCherry (red) localization during syncytial stage and cellularization. Khc-GFP accumulates to the apical domain of Drosophila embryos, Slam-mCherry localizes to the metaphase furrow during syncytial stage and it accumulates to the basal domain during cellularization. The ranges indicate the projection depths of Khc-GFP and Slam$\mathrm{mCherry}$ at different time points. Nuclei (Khc-GFP fluorescence excluded regions) indicate the stages of the embryos. Z-stack size of each step is $1 \mu \mathrm{m}$ and the time interval is $1 \mathrm{~min}$. Scale bar: $10 \mu \mathrm{m}$.

To avoid the potential overexpression of Kinesin-1 by introducing ubi::Khc-GFP transgene in Figure 7, I utilized Kate knock-in allele of Khc Drosophila strain (Gáspár et al., 2017), and checked the localization of Khc-Kate in early embryos by live imaging. Kate fluorescent protein is deriven from red fluorescent protein (RFP). The emergence of new nuclei was defined as $0 \mathrm{~min}$. The result showed that the distribution of Khc-Kate was comparable to the localization of Khc-GFP (Figure 8), Khc-Kate could be found at the cortex of embryos during syncytial (-9-0 min) and cellularization (2-13 min) stages. Taken together, Kinesin-1 localized to the cortex of 
embryos during syncytial and cellularization stages.

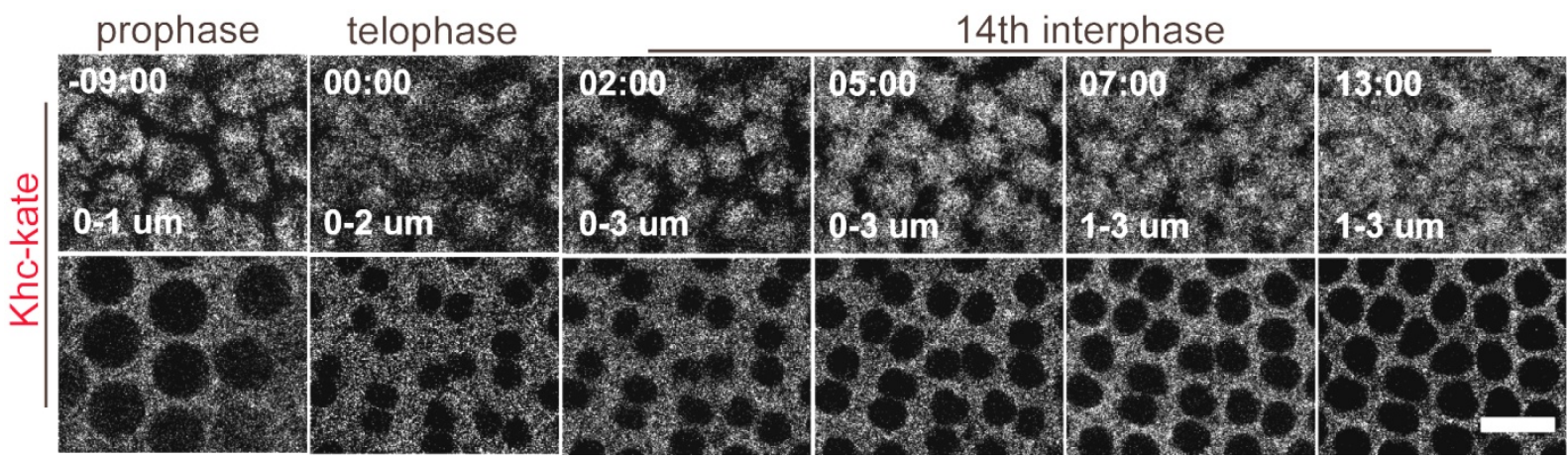

Figure 8 Khc-Kate localizes to the apical domain during syncytial stage and cellularization.

The result shows the localization of Khc-Kate during syncytial (-9-0 min) and cellularization (2-13 min) stages. The first row is Khc-Kate fluorescence at the embryo cortex during syncytial and cellularization stages, the second row indicates nuclei (Khc-Kate fluorescence excluded regions). Ranges indicate projection depths of Khc-Kate. Z-stack size of each step is $1 \mu \mathrm{m}$ and the time interval is $1 \mathrm{~min}$. Scale bar: $10 \mu \mathrm{m}$.

\subsection{Cellularization is impaired in Kinesin-1 RNAi embryos}

The cellularization process in Drosophila embryos starts after finishing 13 nuclear divisions. Along with the membrane invagination during cellularization, Drosophila embryos divide into about 6000 cells. To understand the function of Kinesin-1 during the early embryonic development, I recorded the development of wild type and Kinesin-1 RNAi embryos with differential interference contrast (DIC) microscopy (Figure 9). The DIC microscopy can be used to observe live and unstained Drosophila embryos. The onset of the nuclear elongation during cellularization was defined as the time point $0 \mathrm{~min}$. The result showed that the membrane invagination was visible in wild type embryos at indicated time points. However, this process was affected in Kinesin-1 RNAi embryos, the membrane front was stuck at the surface of Kinesin-1 RNAi embryos. Furthermore, the nuclear elongation was also affected in Kinesin-1 depleted embryos compared to wild type embryos.

In Drosophila embryos, the nuclear elongation happens during cellularization (Fullilove and Jacobson, 1971). However, this process was hampered in Kinesin-1 RNAi embryos, to get a better understanding of the function of Kinesin-1 in the nuclear elongation, quantification of nuclear length in wild type and Kinesin-1 RNAi embryos during cellularization was conducted. The result showed that although the nuclear elongation could be observed in Kinesin-1 RNAi embryos at the onset of cellularization (0-20 min), nuclear shape was affected compared to wild type embryos 
(Figure 10A). The quantification revealed that the nuclear elongation velocity was faster in wild type embryos than in Kinesin-1 RNAi embryos within 20 min (Figure 10B).

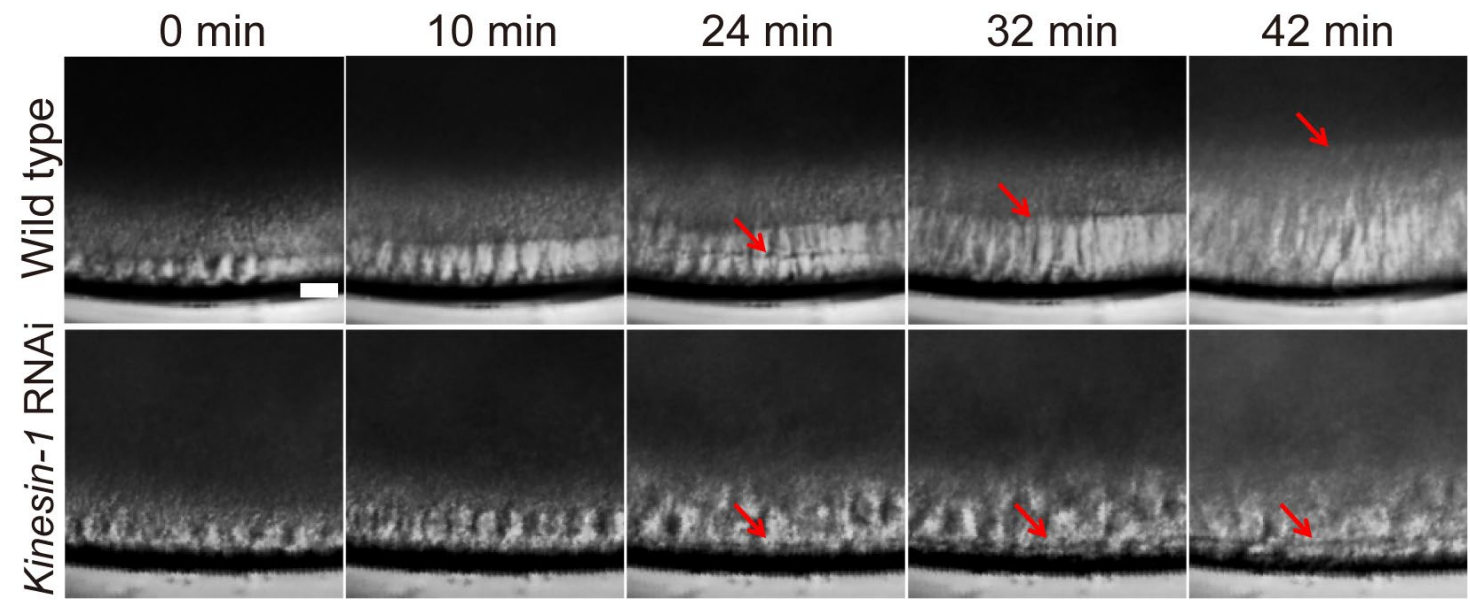

Figure 9 The furrow invagination is compromised in Kinesin-1 RNAi embryos.

The result shows the furrow invagination in wild type and Kinesin-1 RNAi embryos during cellularization. Kinesin-1 is essential for the membrane ingression during cellularization. The onset of the nuclear elongation during cellularization was defined as the time point $0 \mathrm{~min}$. Red arrows indicate the furrow front in both wild type and Kinesin-1 RNAi embryos. Nuclear elongation can be observed during cellularization. The time interval is $2 \mathrm{~min}$. Scale bar: $10 \mu \mathrm{m}$.

Results from DIC microscopy indicated that Kinesin-1 was essential for the membrane invagination and the nuclear elongation during cellularization.
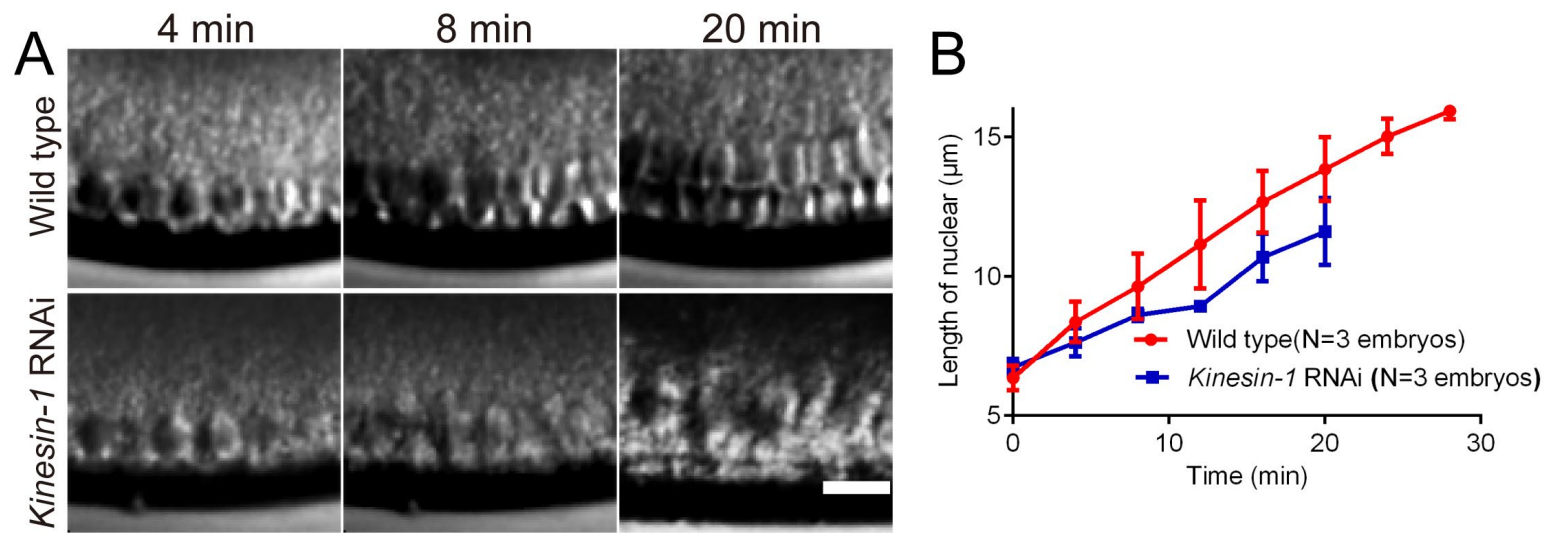

Figure 10 The nuclear elongation is affected in Kinesin-1 RNAi embryos.

(A) Image series from time-lapse DIC microscopy show the nuclear elongation during cellularization in wild type and Kinesin-1 RNAi embryos. (B) Quantification of nuclear length in wild type (red) and Kinesin-1 RNAi (blue) embryos. Three embryos for each genotype and 8 nuclei for each embryo were measured, spots are mean values of nuclear length at the indicated time points. Error bars represent s.e.m. Scale bar: $10 \mu \mathrm{m}$.

\subsection{The cortical polarization is affected in Kinesin-1 RNAi embryos}

Data from DIC microscopy indicated that the membrane invagination was affected in 
Kinesin-1 RNAi embryos. It would be interesting to check the mechanism how Kinesin-1 influenced the membrane invagination during cellularization. The cortex of Drosophila embryos is highly dynamic and it undergoes remodelling over the course of embryonic development (Figure 11). The plasma membrane of Drosophila embryos is organized into two domains during the syncytial interphase: the cap domain and the intercap domain. The cap domain is the region defined by the enrichment of F-actin (Warn et al., 1984), Canoe and ELMO/Sponge complex (an unconventional Guanine nucleotide exchange factor complex). The intercap domain is the region defined by the localization of Slam and Dlg (Geisbrecht et al., 2008; Postner et al., 1992; Schmidt et al., 2018).

During cellularization, the cortex of embryos polarizes into four domains, i.e., apical domain, subapical domain, lateral domain and basal domain (Schmidt and Grosshans, 2018). These domains are marked by distinct proteins. Canoe and ELMO/Sponge complex localize to the subapical domain (Schmidt et al., 2018), Dig and Scribbled localize to the lateral domain (Bilder et al., 2000; David Bilder and Perrimon, 2000), Slam and Myosin II localize to the basal domain (Royou et al., 2003).
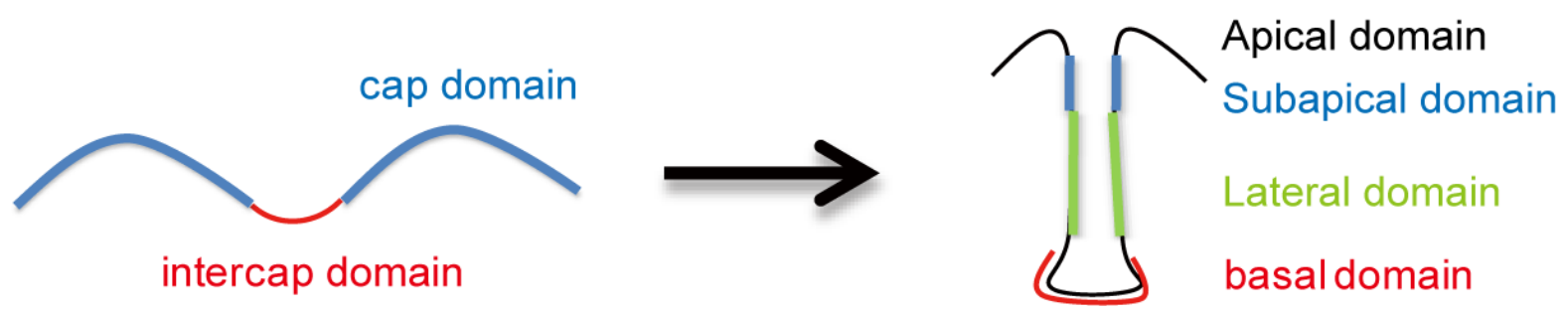

Figure 11 Cortical formation during early embryonic development.

In syncytial blastoderms (from cell cycle 10 to 13), during the interphase, the cortex can be divided into the cap (blue) and the intercap domain (red). Different domains are marked by different proteins. Moesin, toll, and F-actin accumulate to cap domain, Slam and Myosin II localize to the intercap domain. During cellularization, four domains include apical (black), subapical (blue), lateral (green) and basal domains (red) come up. Par-6 and Cdc-42 accumulate to the apical domain; ELMO, Bazooka, and Armadillo localize to the subapical domain; Discs large (Dlg), Lethal giant larva (Lgl) and E-Cadherin can be found at the lateral domain; Slam, Myosin II and Amphyphisin accumulate to the basal domain.

In agreement with my previous colleague (Winkler et al., 2015), my results (Figure 9 and Figure 10) indicate that Kinesin-1 is indispensable for the cellularization of Drosophila embryos, but the mechanism how Kinesin-1 influences cellularization is not clear. To gain insights into the mechanism of how Kinesin-1 contributes to cellularization, I checked Slam dynamics in wild type and Kinesin-1 RNAi embryos. Slam protein localizes to the intercap domain during the syncytial interphase and 
moves to the basal domain during cellularization. Slam is essential for the membrane invagination during cellularization via recruiting RhoGEF2 to the furrow canal (Lecuit et al., 2002; Wenzl et al., 2010).

To check the localization of Slam in Kinesin-1 RNAi embryos, I imaged the localization of GFP-Slam during syncytial (0-3 min) and cellularization (7-16 min) stages in wild type and Kinesin-1 RNAi embryos (Figure 12). The onset of the mitosis in cell cycle 13 was defined as $0 \mathrm{~min}$. The result showed that, at $0 \mathrm{~min}$, GFP-Slam localized to the basal domain of metaphase furrow $(4-6 \mu \mathrm{m})$ during mitosis in wild type and Kinesin-1 RNAi embryos, suggested that Kinesin-1 depletion did not affect the Slam localization at the metaphase furrow. At the onset of cellularization (9 $\mathrm{min}$ ), Slam was sharply restricted to the forming membrane in wild type embryos but not in Kinesin-1 RNAi. The result indicated that the localization of GFP-Slam at the basal domain was affected in Kinesin-1 RNAi embryos during cellularization.

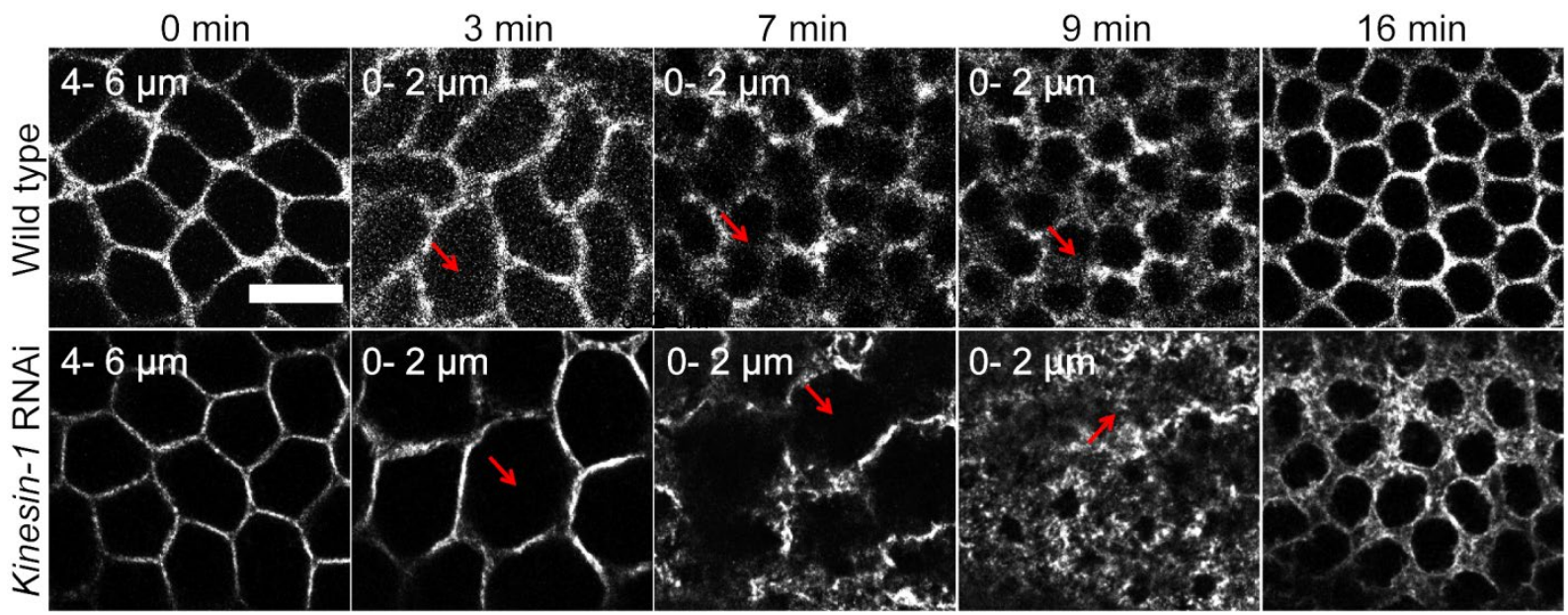

Figure 12 GFP-Slam is mislocalized during cellularization in Kinesin-1 RNAi embryos.

Images from time-lapse recordings of GFP-Slam in wild type and Kinesin-1 RNAi embryos during syncytial (0-3 $\mathrm{min}$ ) and cellularization (7-16 $\mathrm{min}$ ) stages. Kinesin-1 RNAi has a strong effect on GFPSlam localization when the new membrane forms. Ranges indicate projection depths of GFP-Slam. Zstack size of each step is $1 \mu \mathrm{m}$ and the time interval is $1 \mathrm{~min}$. Red arrows indicate positions where new membrane forms and GFP-Slam accumulates. Scale bar: $10 \mu \mathrm{m}$.

The previous publication indicated that Slam is required for slam RNA localization (Yan et al., 2017). slam RNA co-localizes with Slam protein at the basal domain in wild type embryos during cellularization (Yan et al., 2017). To check whether slam RNA localization was affected in Kinesin-1 RNAi embryos, immunostaining of Slam protein and in situ hybridization of slam RNA were performed in wild type and Kinesin-1 RNAi embryos. The result showed that during cellularization, slam RNA and Slam protein colocalized to the basal domain in wild type embryos, whereas slam RNA and Slam protein were mislocalized in Kinesin-1 RNAi embryos. slam RNA 
and Slam protein were stuck at the surface of Kinesin-1 RNAi embryos (Figure 13). Taken together, live images of GFP-Slam and immunostaining of Slam protein in wild type and Kinesin-1 RNAi embryos indicated that Kinesin-1 was required for the localization of Slam protein during cellularization.
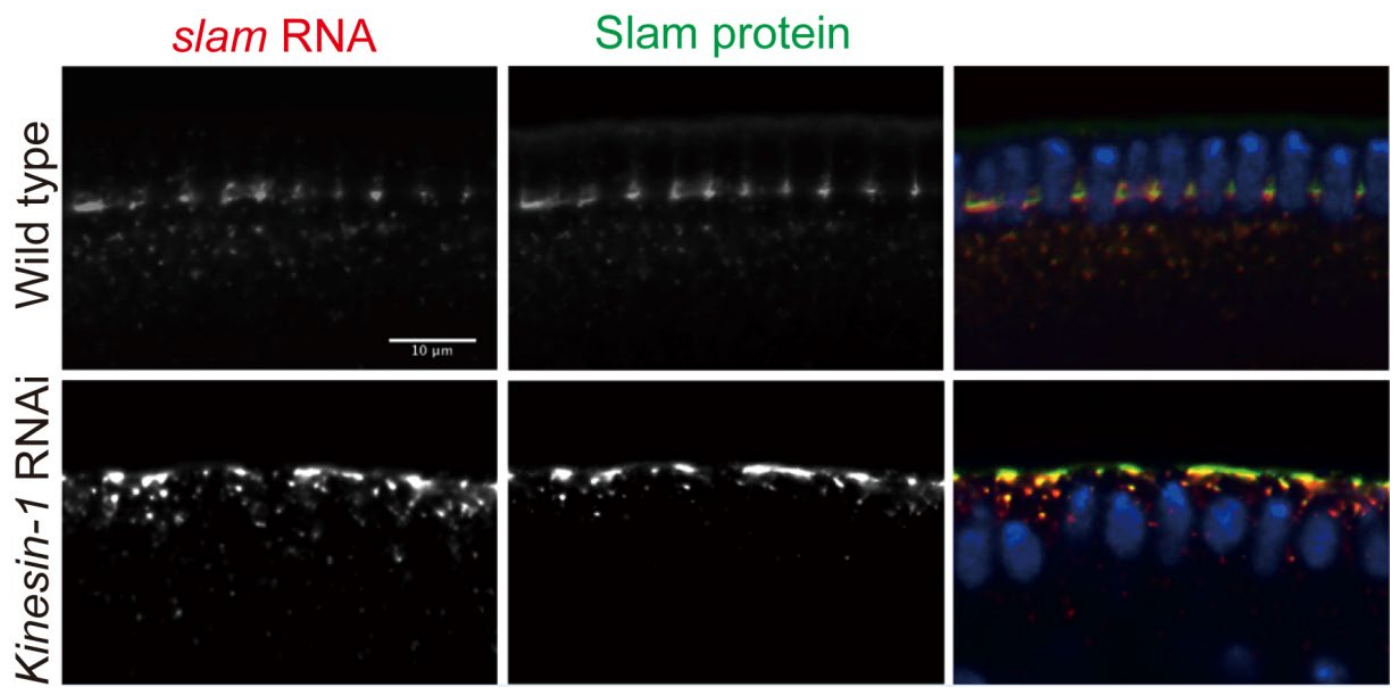

Figure 13 The slam RNA localization is affected in Kinesin-1 RNAi embryos during cellularization.

The slam RNA and protein localization in wild type and Kinesin-1 RNAi embryos. In wild type embryos, slam RNA (red) and Slam protein (green) accumulate to the basal domain, while slam RNA and Slam protein are mainly stuck at the surface of Kinesin-1 RNAi embryos. Scale bar: $10 \mu \mathrm{m}$.

The mislocalization of Slam indicated that the basal domain was affected in Kinesin-1 depleted embryos during cellularization. Kinesin-1 is a motor protein, which plays an important role in cortical components translocation during the embryonic axes formation (Brendza et al., 2002; Januschke et al., 2002).
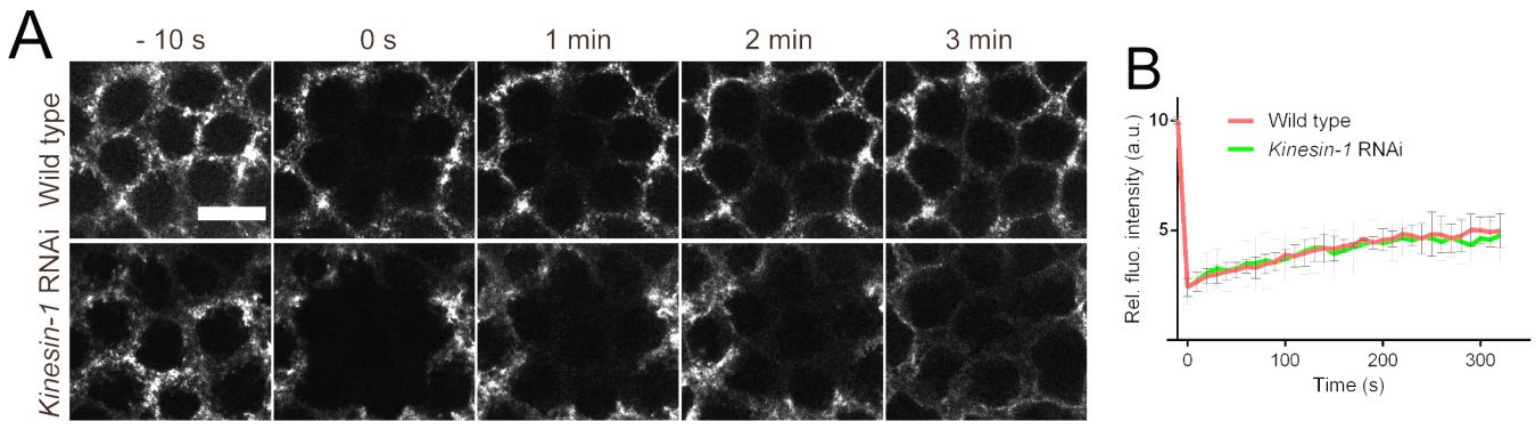

Figure 14 Slam dynamics are comparable in wild type and Kinesin-1 RNAi embryos at the onset of cellularization.

(A) Live images of GFP-Slam dynamics in FRAP experiments in wild type and Kinesin-1 RNAi embryos during cellularization. -10 s means $10 \mathrm{~s}$ before photo-bleaching. (B) Quantification of GFPSlam fluorescence recovery velocity in wild type (red) and Kinesin-1 RNAi (green) embryos. Relative fluorescent intensities of GFP-Slam at the bleached region are measured at the indicated time points in wild type and Kinesin-1 RNAi embryos, 3 embryos are measured in wild type and Kinesin-1 RNAi respectively. Fluorescent intensity at the bleached region is recorded every $10 \mathrm{~s}$. Z-stack size of each step is $1 \mu \mathrm{m}$. Error bars represent s.e.m. Scale bar: $10 \mu \mathrm{m}$. 
It is reasonable to check whether the disruption of cortical polarization was due to the defects of protein transport dynamics. To clarify this, dynamics of GFP-Slam in wild type and Kinesin-1 RNAi embryos during cellularization were checked via fluorescence recovery after photo-bleaching (FRAP) experiments. GFP-Slam fluorescence recovery dynamics in wild type and Kinesin-1 RNAi embryos were recorded (Figure 14A). As Slam has high mobility at the beginning of cellularization and Slam mobility is much lower at the middle of cellularization (Acharya et al., 2014), I performed the FRAP experiments at the onset of cellularization. The fluorescent intensity of GFP-Slam was recorded every $10 \mathrm{~s}$ and the time when the photobleaching started was defined as $0 \mathrm{~s}$. Quantification of GFP-Slam recovery dynamics revealed that GFP-Slam recovery speed was comparable in wild type and Kinesin-1 RNAi embryos (Figure 14B), indicated that the mislocalization of Slam during cellularization in Kinesin-1 RNAi embryos was not induced by the defects of Slam dynamics at the new membrane.

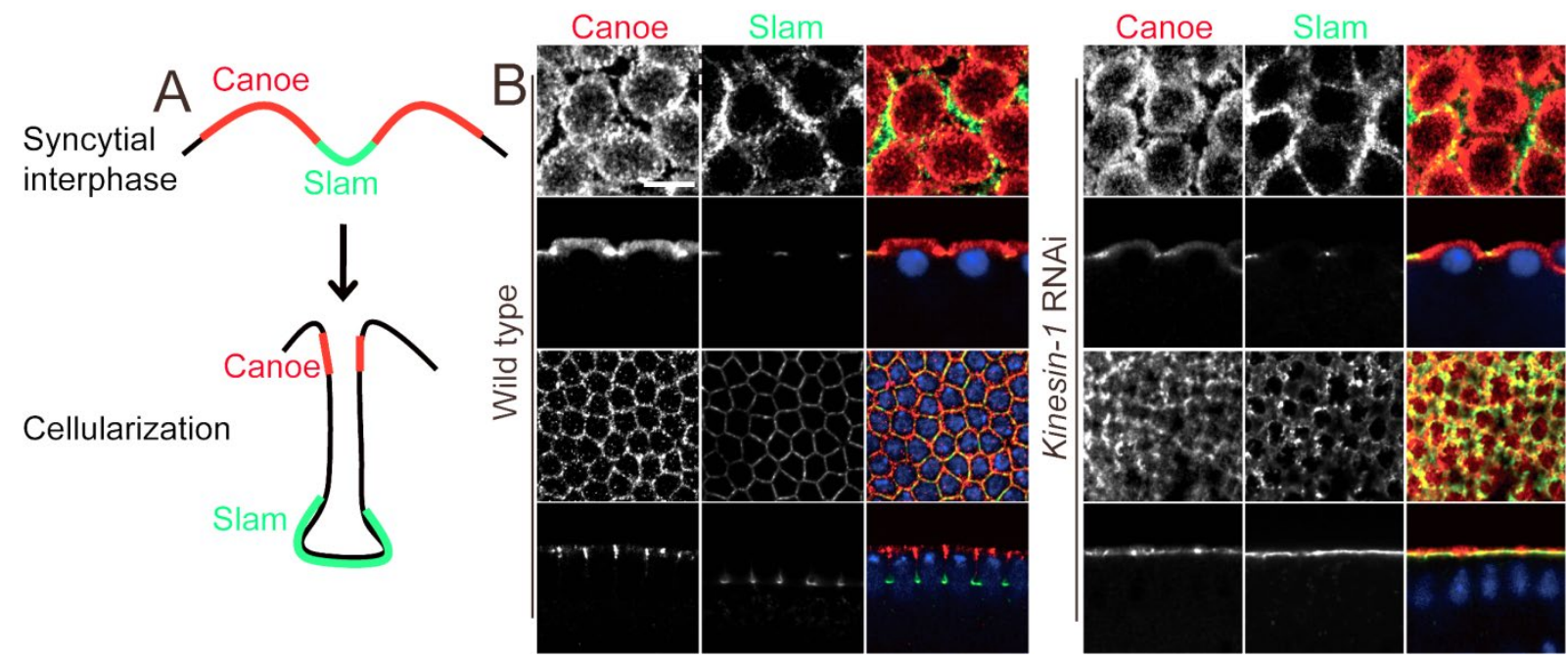

Figure 15 The formation of subapical domain is affected in Kinesin-1 RNAi embryos.

(A) Schematic of Canoe (red) and Slam (green) localization in syncytial and cellularization stages. During the syncytial stage, Canoe localizes to the cap domain, Slam localizes to the intercap domain. During cellularization, Canoe accumulates to the subapical domain, and Slam localizes to the basal domain. (B) Images of Canoe staining in wild type and Kinesin-1 RNAi embryos during the syncytial interphase (first and second rows, surface view and section view respectively) and cellularization (third and fourth rows, surface view and section view respectively). The localization of Canoe (red) and Slam (green) are not affected in Kinesin-1 RNAi embryos during syncytial stage. Canoe and Slam localize to the subapical domain and basal domain respectively during cellularization in wild type, while both of them are stuck at the surface of Kinesin-1 RNAi embryos during cellularization. Scale bar: $10 \mu \mathrm{m}$.

Different domains come up at different time points during cellularization. For example, at the onset of cellularization, the newly formed subapical domain comes up slightly later than the basal domain (Schmidt et al., 2018; Schmidt and Grosshans, 2018), it 
is worth to check the formation of different domains in Kinesin-1 RNAi embryos.

Canoe is a actin-binding protein, which localizes to the subapical domain, Canoe is essential for the subapical localization of Bazooka and E-Cadherin (Choi et al., 2013). To clarify whether Kinesin-1 depletion only compromised the basal domain formation or it also affected other domains formation during cellularization, I checked the localization of Canoe in Kinesin-1 RNAi embryos by immunostaining (Figure 15). Immunostaining results showed that, in both wild type and Kinesin-1 RNAi syncytial blastoderms, Canoe accumulated to the cap domain and Slam was restricted to the intercap domain. However, the localization of Canoe was affected in Kinesin-1 RNAi embryos during cellularization. In wild type embryos, Canoe localized to the subapical domain and Slam enriched at the basal domain during cellularization, the separation between Canoe and Slam was clear. However, in Kinesin-1 RNAi embryos, Canoe and Slam accumulated at the cortex of embryos without clear separation during cellularization. The result indicated that Kinesin-1 was required for the Canoe localization and the subapical domain formation.
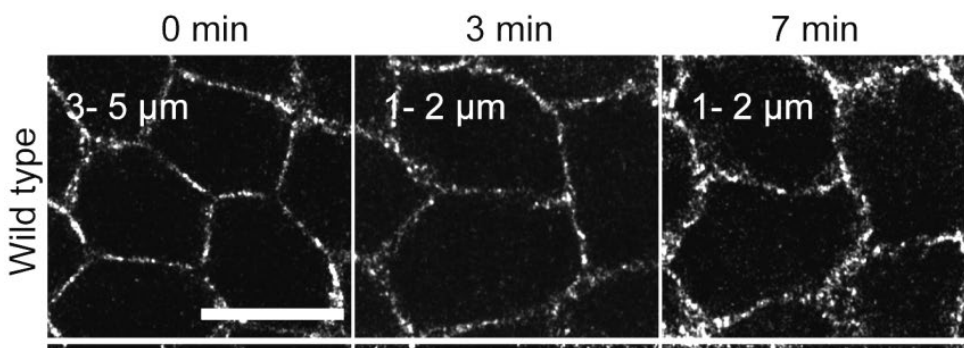

9 min $16 \min$

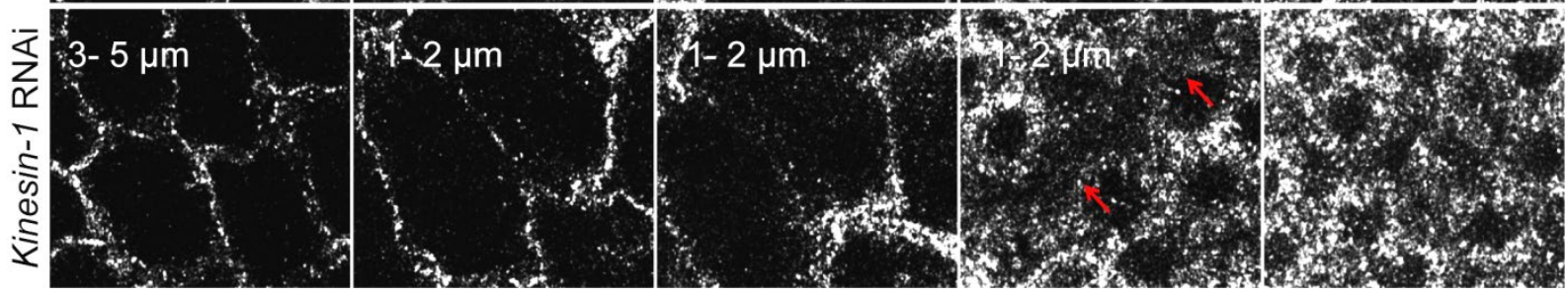

Figure 16 Subapical domain is influenced in Kinesin-1 RNAi embryos.

Images from time-lapse recordings of Canoe-YFP in wild type and Kinesin-1 RNAi embryos during syncytial (0-7 $\mathrm{min})$ and cellularization (9-16 $\mathrm{min})$ stages. With new membrane formation during cellularization, Canoe-YFP accumulates sharply at the subapical domain in wild type, while the CanoeYFP accumulation is not so restricted in Kinesin-1 RNAi embryos. Ranges indicate projection depths of Canoe-YFP. Z-stack size of each step is $1 \mu \mathrm{m}$ and the time interval is $1 \mathrm{~min}$. Arrows indicate localization of Canoe-YFP at the new membrane. Scale bar: $10 \mu \mathrm{m}$.

To get detailed information about the localization of Canoe in syncytial and cellularization stages in Kinesin-1 RNAi embryos, I detected Canoe-YFP localization with live imaging (Figure 16). The onset of the mitosis of cell cycle 13 was defined as $0 \mathrm{~min}$. The result showed that the Canoe-YFP distribution was comparable in cell cycle 13 in both wild type and Kinesin-1 RNAi embryos. During mitosis (0-7 min), 
Canoe-YFP localized to the metaphase furrow in both wild type and Kinesin-1 RNAi embryos. During cellularization (9-16 min), in Kinesin-1 depleted embryos, although the enrichment of Canoe-YFP at the new membrane was observed, it was not as restricted as in wild type. Taken together, results from live imaging and immunostaining of Canoe indicated that the subapical domain was also compromised in Kinesin-1 RNAi embryos.

Canoe moves from the cap domain to the subapical domain when embryos transit from the syncytial stage to cellularization stage. It has been reported that the subapical localization of Canoe depends on the ELMO/Sponge complex (Schmidt et al., 2018). ELMO/Sponge complex is required for actin cap and metaphase furrow formations (Postner et al., 1992). Interestingly, ELMO/Sponge complex also migrates from the cap domain to the subapcial domain during the transition from syncytial stage to cellularization (Figure 17A).

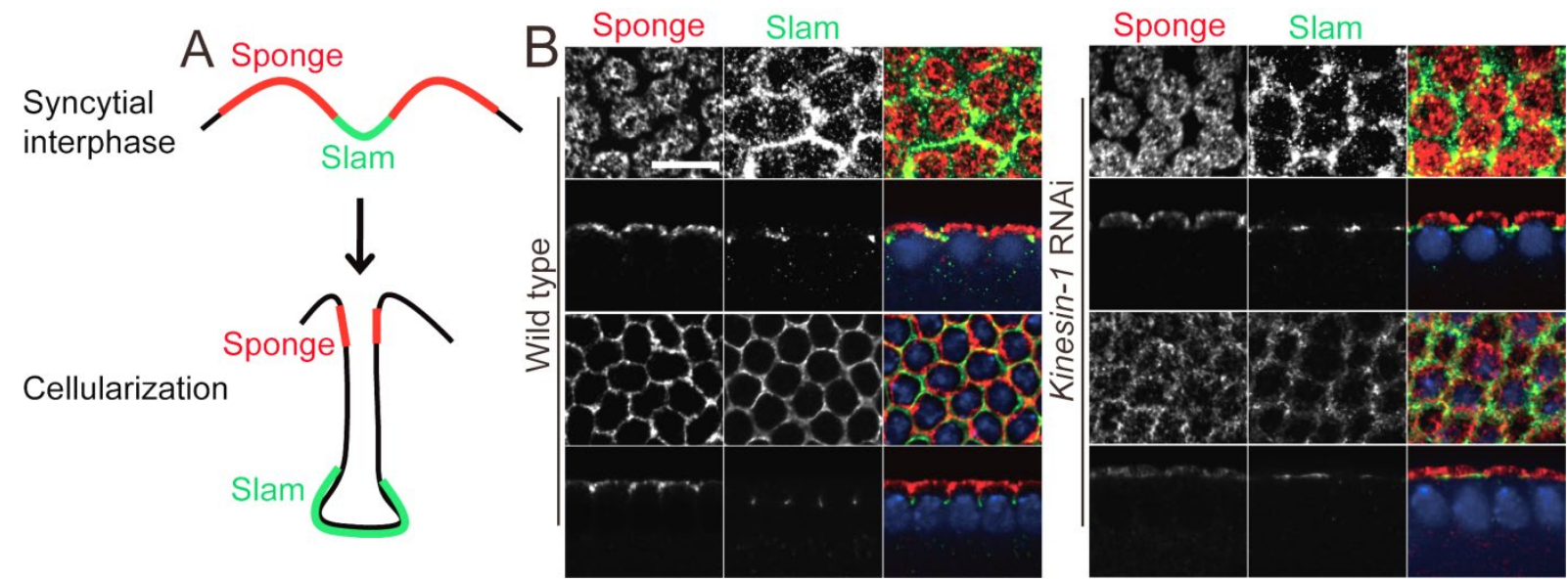

Figure 17 The distribution of Sponge is affected in Kinesin-1 RNAi embryos during cellularization.

(A) Schematic of Sponge (red) and Slam (green) localization in syncytial and cellularization stages. During the syncytial stage, Sponge localizes to the cap domain, Slam localizes to the intercap domain. During cellularization, Sponge migrates to the subapical domain, Slam localizes to the basal domain. (B) Staining of Sponge (red) and Slam (green) in both wild type and Kinesin-1 RNAi in syncytial (the first and second rows, surface view and section view respectively) and cellularization (the third and fourth rows, surface view and section view respectively) stages. The Sponge localization is affected in Kinesin-1 RNAi embryos during cellularization. Scale bar: $10 \mu \mathrm{m}$.

As ELMO/Sponge complex localizes to the upstream of Canoe, it is reasonable to identify whether Kinesin-1 depletion influenced the localization of ELMO/Sponge complex and induced the mislocalization of Canoe. To check the localization of ELMO/Sponge complex, I immunostained Sponge protein in wild type and Kinesin-1 depleted embryos. The result indicated that, during the syncytial interphase of wild type and Kinesin-1 RNAi embryos, Sponge mainly accumulated to the cap domain, 
surrounded by Slam at the intercap domain. During cellularization, in wild type embryos, Sponge moved and enriched to the subapical domain, Slam localized to the basal domain, the separation between Sponge and Slam was clear. However, the localization of Sponge was affected in Kinesin-1 RNAi embryos. Sponge and Slam proteins were stuck at the surface of embryos, the separation between Sponge and Slam was not clear in Kinesin-1 RNAi embryos (Figure 17B).

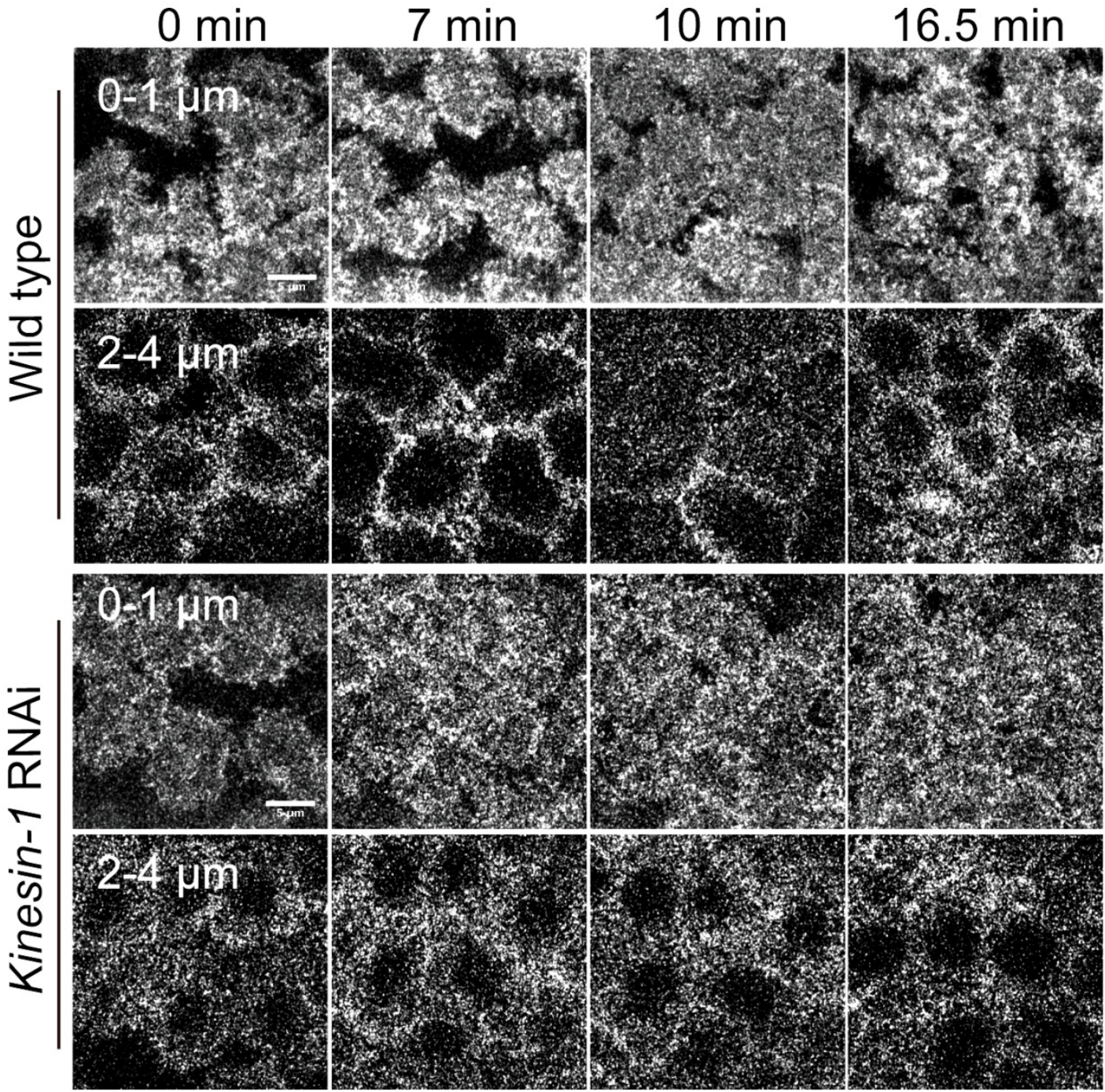

Figure 18 The ELMO-GFP localization is affected in Kinesin-1 RNAi embryos.

The live images show the ELMO-GFP localization during the syncytial stage (0-10 min) and cellularization (16.5 min) in both wild type and Kinesin-1 RNAi embryos. The onset of the interphase of cell cycle13 was defined as $0 \mathrm{~min}$. The ranges indicate the projection depths of ELMO-GFP. The first and third rows are the ELMO-GFP fluorescence on the cortex $(0-1 \mu \mathrm{m})$, the second and fourth rows are the ELMO-GFP fluorescence at the edge of the cap domain $(2-3 \mu \mathrm{m})$. Z-stack size of each step is $1 \mu \mathrm{m}$ and the time interval is $0.5 \mathrm{~min}$. Scale bar: $5 \mu \mathrm{m}$.

To gain insights into the distribution of ELMO/Sponge complex in Kinesin-1 RNAi embryos, I recorded the localization of ELMO-GFP by live imaging (Figure 18). The result showed that, in wild type embryos, ELMO-GFP mainly accumulated to the cap 
domain during the interphase of cell cycle 13, formed disc-like structures at the edge of cap domain. However, there was no restricted enrichment of ELMO-GFP at the rim of cap domain in Kinesin-1 RNAi embryos. During cellularization, in wild type, ELMOGFP could be observed not only at the apical domain but also at the subapical domain. However, ELMO-GFP distributed evenly on the cortex in Kinesin-1 RNAi embryos (Figure 18). Taken together, results from live imaging and immunostaining indicated Kinesin-1 was essential for the localization of ELMO/Sponge complex.

\subsection{Centrosomes and microtubules are not affected in Kinesin-1 depleted embryos}

The centrosome, as microtubule organizing center (MTOC) and the minus end of microtubules, plays a very vital role in directed transport, which is important for the cortical components localization (Sanchez and Feldman, 2017). Furthermore, centrosome is capable to induce cortical polarization (Cowan and Hyman, 2004).

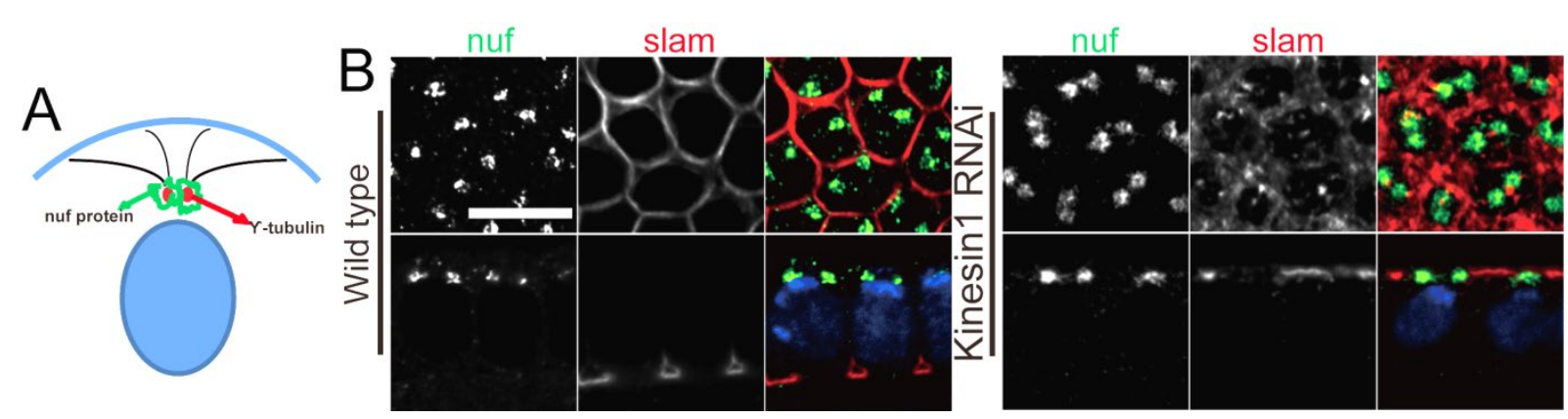

Figure 19 Minus end direct transport towards centrosome is largely normal in Kinesin-1 RNAi embryos.

(A) Schematic of Nuf and y-tubulin localization. Nuf localizes arround centrosomes, y-tubulin as a subunit of centrosomes, localizes to the centrosome. (B) Nuf (green) and Slam (red) Staining in Kinesin-1 RNAi and wild type embryos during cellularization. Nuclei are stained with DAPI. Scale bar: $10 \mu \mathrm{m}$.

The previous publication reported that recycling endosome is connected to centrosomes in syncytial blastoderms, recycling endosome is required for the Iocalization of Slam during cellularization (Acharya et al., 2014). I wondered whether the mislocalization of Slam in Kinesin-1 RNAi embryos was induced by the defects of recycling endosome. Nuclear fallout (Nuf) is a cytoplasmic, coiled-coil protein that acts as an adaptor between Rab11 and motor proteins in the recycling endosome pathway (Pereira and Schiebel, 1997; Raff and Glover, 1989; Riggs et al., 2007). Nuf accumulates arround centrosomes (Figure 19A). Nuf, as a marker protein of recycling 
endosome was used to check whether recycling endosome was affected in Kinesin-1 RNAi embryos. The result showed that recycling endosomes were enlarged in the Kinesin-1 RNAi embryos, revealed that recycling endosomes were affected because of Kinesin-1 depletion, but the position and the number of Nuf staining in Kinesin-1 RNAi embryos were normal compared to wild type (Figure 19B).

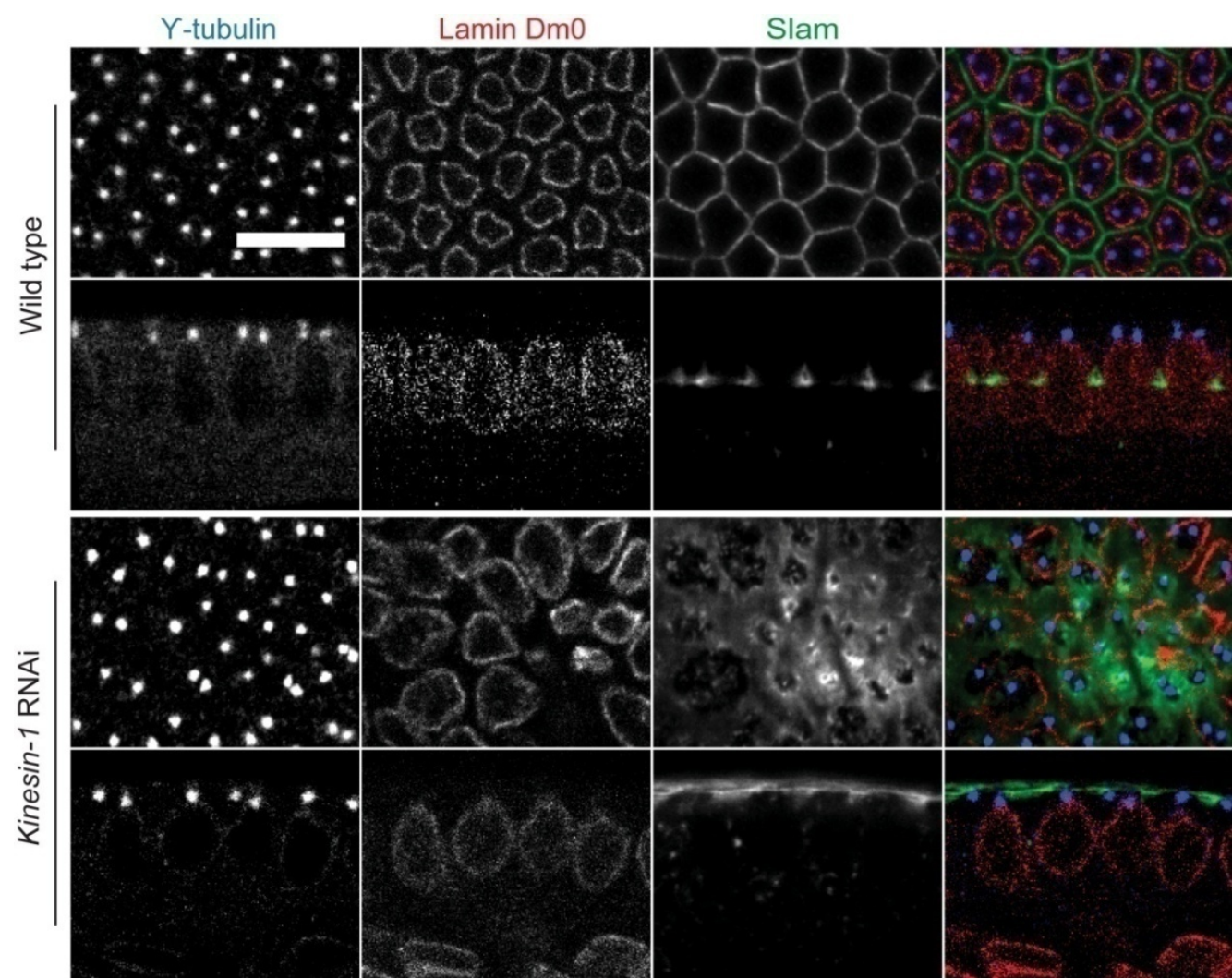

Figure 20 Centrosomes are not affected in Kinesin-1 RNAi embryos.

The result shows stainings of $\mathrm{Y}$-tubulin (blue), Lamin Dm0 (red) and Slam (green) in wild type and Kinesin-1 RNAi embryos during cellularization. The localization and the number of centrosomes that above nucleus are comparable between wild type and Kinesin-1 RNAi embryos during cellularization. Slam protein localizes to the basal domain during cellularization in wild type embryos. Lamin Dm0, as a marker of nuclei, specifically localizes to the nuclear membrane. Scale bar: $10 \mu \mathrm{m}$.

As centrosomes are crucial for cortical components localization and cortical polarization, it is worth to check whether defects of cellularization was induced by disruption of centrosomes. Immunostaining of $\mathrm{y}$-tubulin was performed to check whether centrosomes were affected in the Kinesin-1 RNAi embryos, as y-tubulin subunit is a main component of centrosomes (Pereira and Schiebel, 1997; Schulze and Kirschner, 1986). Lamin Dm0 and Slam were stained to indicate nuclei and basal domain respectively. Lamin Dm0 localizes to the nuclear membrane (Smith et al., 1987).

The result showed that although the localization of Slam was affected in Kinesin-1 RNAi embryos, the number of centrosomes above nuclei was normal from both top 
view and section view in Kinesin-1 RNAi embryos compared to wild type, proved that Kinesin-1 depletion did not affect centrosomes (Figure 20). Overall, the recycling endosomes in wild type and Kinesin-1 RNAi embryos were comparable.

Microtubules are important for both dividing and non-dividing cells. In dividing cells, microtubules form mitotic spindles for chromosomes segregation and orient the plane of cleavage (Kapitein et al., 2005). In non-dividing cells, microtubules organize cytoplasm and organelles, such as the position of nucleus and mitochondria (Pilling et al., 2006). Astral microtubules (+)-ends toward out to the cap region during the interphase (Winkler et al., 2015), Kinesin-1 binds to microtubules, the function of Kinesin-1 depends on microtubules, also the organization of microtubules is rely on Kinesin-1 (Coravos and Martin, 2016). Thus, it is reasonable to check whether microtubules were disrupted in Kinesin-1 RNAi embryos.

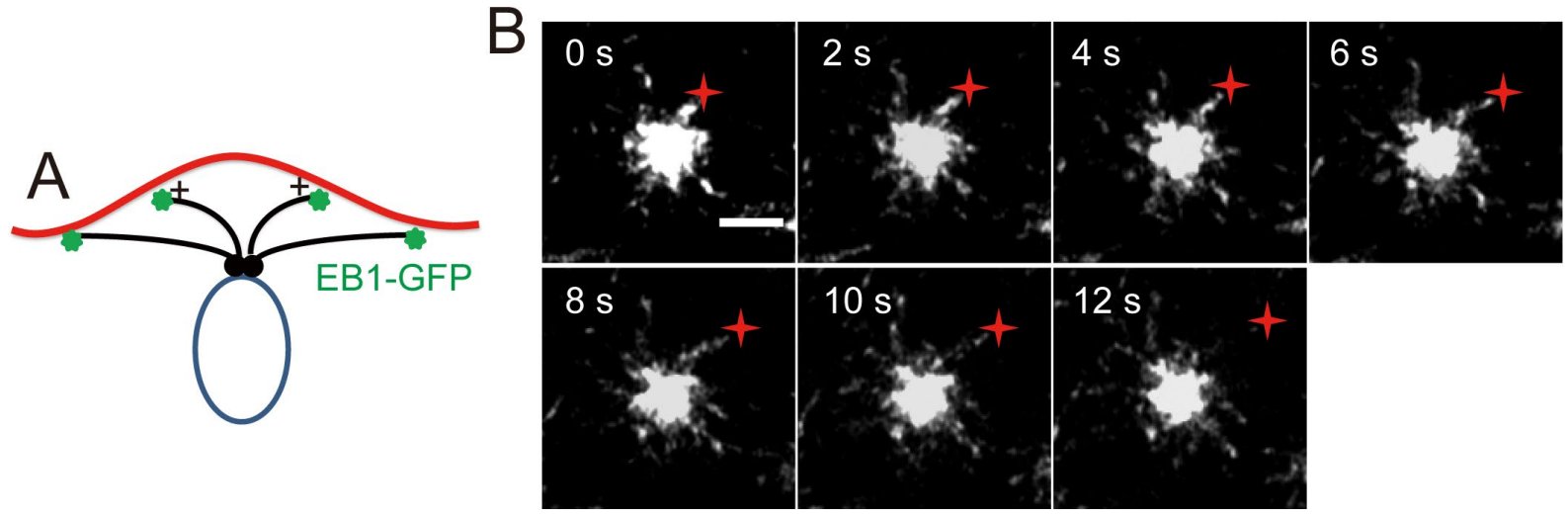

Figure 21 Microtubule is a dynamic structure during interphase.

(A) Schematic of the EB1-GFP localization. EB1-GFP (green) localizes to the plus end of microtubules, the movement of EB1-GFP reflects the polymerization of microtubules. (B) The live images show the localization of EB1-GFP at different time points. Red stars indicate different positions of the EB1-GFP cluster at different time points. Scale bar: $2 \mu \mathrm{m}$.

EB1 is a plus end tracking protein and it accumulates to the growing end of microtubules (Bouissou et al., 2014; L. K. Su et al., 1995). GFP-labeled end binding protein 1 (EB1-GFP) was utilized to track the polymerization of microtubules. The movement of EB1-GFP was recorded every $2 \mathrm{~s}$ (Figure 21). The result showed that the majority of EB1-GFP accumulated to centrosomes, some EB1-GFP clusters moved away from centrosomes during interphase. The movement of EB1-GFP clusters indicated the polymerization of microtubules at their plus end. EB1-GFP fluorescence was stable, it could be tracked in several seconds. The route (Figure $22 \mathrm{~A}$ ) and the velocity (Figure 22B) of EB1-GFP clusters movement were figured out with coordinates at different time points. The velocity quantification revealed that there was no significant difference in EB1-GFP movement between wild type and 
Kinesin-1 RNAi embryos. This result indicated that polymerization dynamics of microtubules were not hampered in the Kinesin-1 RNAi embryos.

Quantification of the EB1-GFP movement in wild type and Kinesin-1 RNAi embryos showed that the polymerization of microtubules was not affected, but the distribution of plus ends of microtubules has not been detected. It has been reported that plus ends of microtubules and F-actin filaments interact intensively in multiple cellular processes (Coles and Bradke, 2015; Dogterom and Koenderink, 2019b).
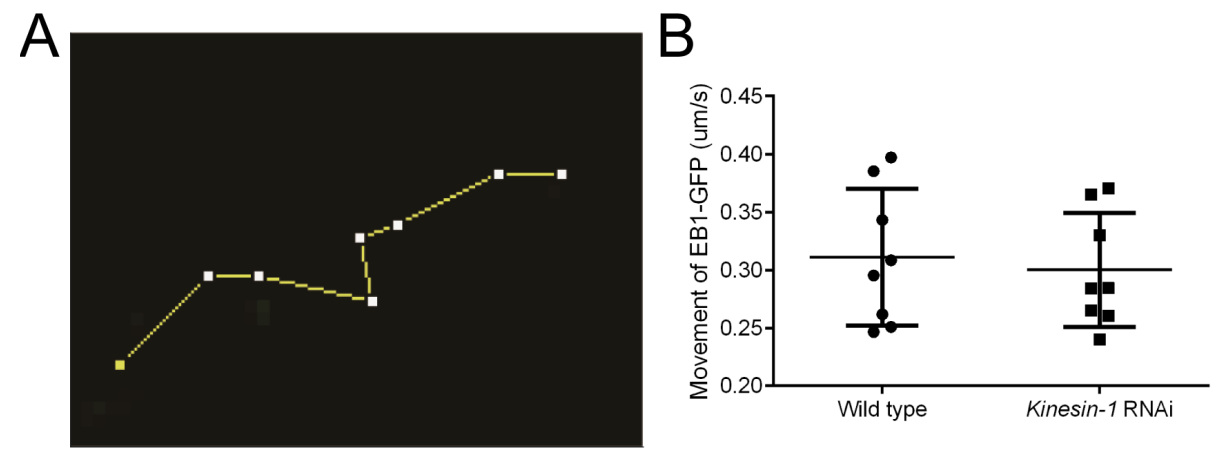

Figure 22 The polymerization of microtubules is normal in Kinesin-1 depleted embryos.

(A) Example of EB1-GFP cluster movement route in $14 \mathrm{~s}$. (B) The velocity of EB1 movement in wild type and Kinesin-1 RNAi embryos. Although the speed of EB1-GFP movement is diverse, the average velocity is comparable between wild type and Kinesin-1 RNAi embryos. Spots indicate average speed of EB1 clusters movement in wild type and Kinesin-1 RNAi embryos. The P-value is calculated from the paired Student's t-test.

Thus, I checked dynamics of microtubules and F-actin filaments simultaneously by live imaging. Moesin is the only ERM protein in Drosophila, which is essential for cortical domains maintenance and cortical stability (Karagiosis and Ready, 2004), Moesin and F-actin localize to the cap domain during the interphase. I used embryos expressing Moesin-RFP and EB1-GFP to visualize the distribution of F-actin filaments and plus ends of microtubules respectively.

The result showed that in wild type embryos, during interphase, EB1-GFP and Moesin-RFP mainly accumulated at the cortex especially at the cap domain, EB1GFP and Moesin-RFP had an overlap at the cap domain at the indicated time points (Figure 23). The movement of EB1-GFP cluster on F-actin cap could be observed as well (arrows indicate).

To quantify the distribution of plus ends of microtubules, I measured fluorescent intensities of EB1-GFP and Moesin-RFP at the cap domain and intercap domain and calculated the distribution ratio of EB1-GFP and Moesin-RFP between the cap $\left(\rho^{i}\right)$ domain and the intercap $\left(\rho^{\circ}\right)$ domain during interphase (Figure 24A). The 
quantification showed that distribution ratios of Moesin-RFP $\left(\rho^{i} / \rho^{\circ}\right)$ and EB1-GFP $\left(\rho^{i} / \rho^{\circ}\right)$ were about 2.4 and 2.8 respectively, indicated that F-actin mainly accumulated to the cap domain, and plus ends of microtubules mainly localized under the cap domain during interphase (Figure 24B,C).

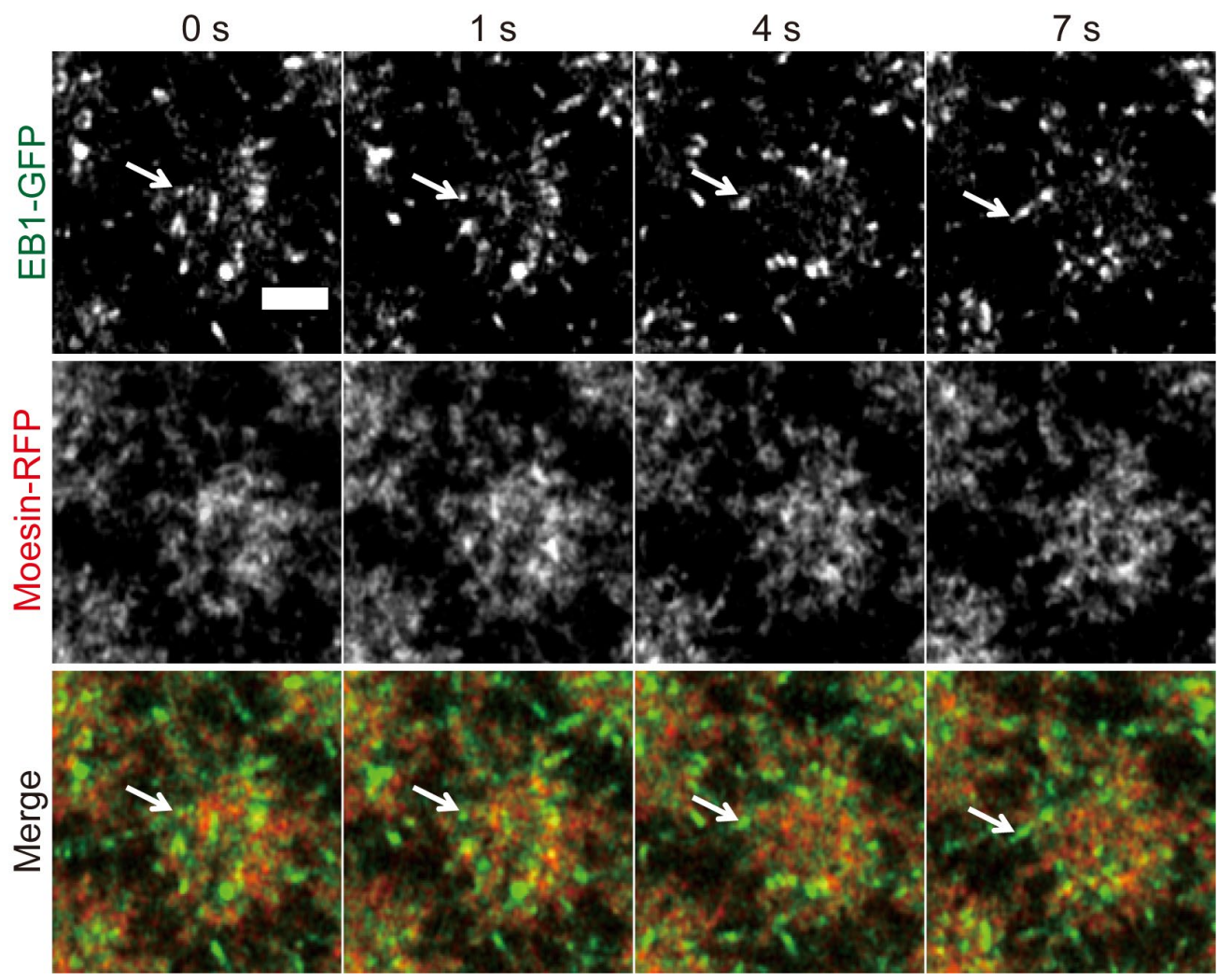

Figure 23 EB1 and Moesin mainly localize to the cap domain in wild type embryos.

The live images show the localization of EB1-GFP (green) and Moesin-RFP (red) at the indicated time points. EB1-GFP (green) and Moesin-RFP have an overlap at the cap region at different time points in wild type embryos. Moesin-RFP indicates the cap domain during interphase. White arrows indicate positions of the EB1-GFP cluster at different time points during interphase. Scale bar: $2 \mu \mathrm{m}$.

It would be interesting to check whether the distribution of plus ends of microtubules is affected in Kinesin-1 RNAi embryos, and mis-organization of microtubules plus ends might be one explanation for the disruption of cortical polarity.

\subsection{The differentiation of cap and intercap domains is not affected} in Kinesin-1 RNAi embryos

Above results indicated that during cellularization, (1) Kinesin-1 localized to the cortex of embryos, (2) the localization of Slam, Canoe and ELMO/Sponge complex were affected in Kinesin-1 depleted embryos, and (3) the localization of ELMO-GFP at the cap domain was affected as well in Kinesin-1 RNAi embryos during the interphase of syncytial blastoderms. The cortical differentiation in Kinesin-1 RNAi 
embryos was affected. Since Kinesin-1 localized to the cap domain during the interphase and it influenced the translocation of Canoe and ELMO/Sponge from cap domain to subapical domain during cellularization, It is reasonable to check the differentiation of cap domain and intercap domain during the interphase of Kinesin-1 RNAi syncytial blastoderms.

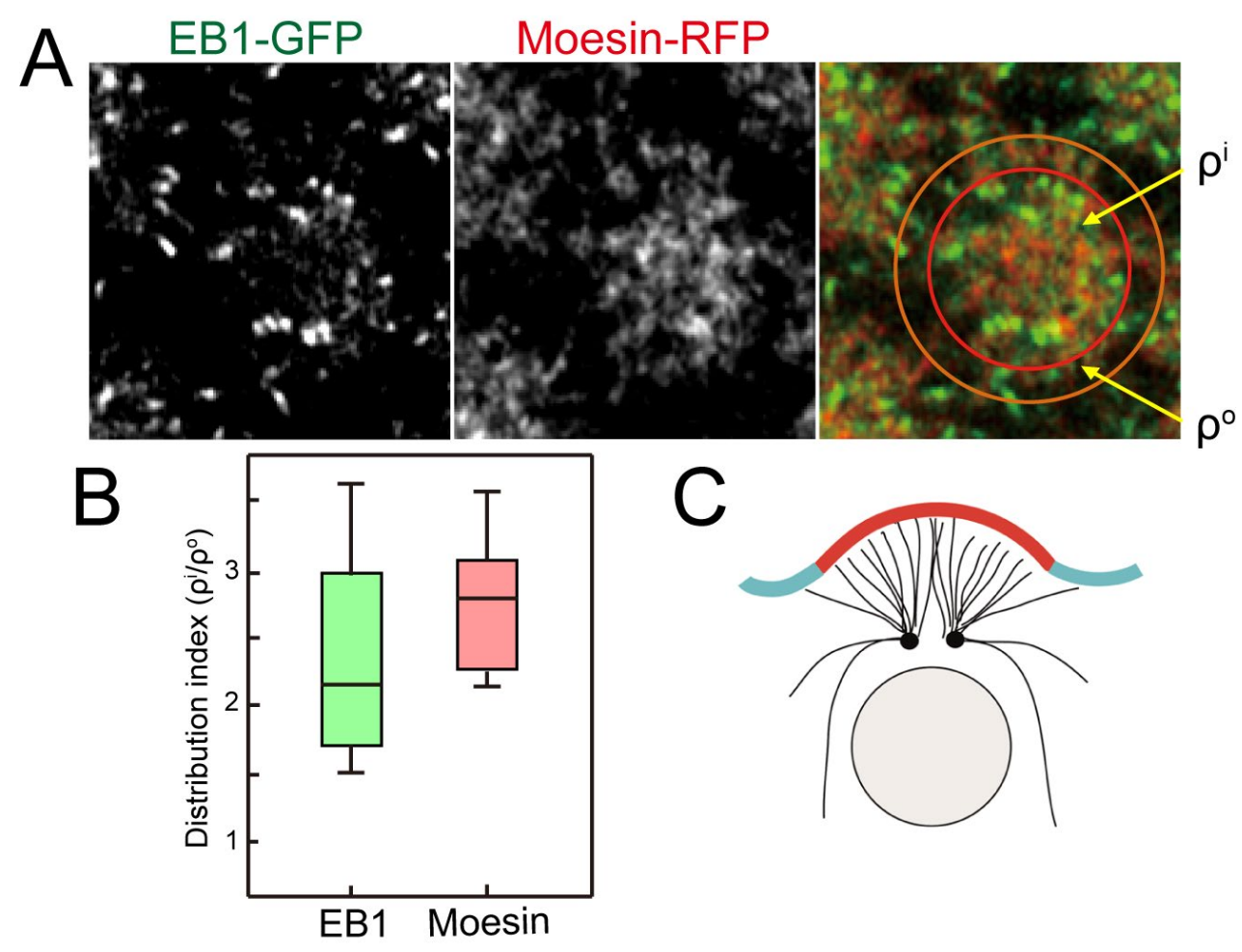

Figure 24 EB1 and Moesin mainly accumulated at the cap domain.

(A) EB1-GFP (green) and Moesin-RFP (red) have an overlap at the cap region, $\rho^{i}$ indicates the distribution of EB1 and Moesin fluorescent intensities at the cap region, $\rho^{\circ}$ indicates the distribution of EB1 and Moesin fluorescent intensities at the intercap region. (B) Distribution index of EB1-GFP (green) and Moesin-RFP (red) fluorescent intensity between the cap and the intercap region. EB1GFP and Moesin-RFP fluorescent intensities in three embryos (four measurements for each embryo) are measured. Error bars indicate the minimum and maximum values, edges of boxes represent 25th and 75th percentile values respectively. (C) Schematic of plus ends of microtubules (black) distribution.

During the interphase of syncytial blastoderms, the cortex of embryos divides into cap domain and intercap domain. To get detailed information about whether the intercap domain was affected in Kinesin-1 RNAi embryos, I conducted immunostainings of Dlg and Slam in wild type and Kinesin-1 RNAi embryos (Figure 25). The result showed that during the syncytial interphase, Dlg and Slam accumulated to the intercap domain in wild type and Kinesin-1 RNAi embryos. The result indicated that intercap region was not affected in Kinesin-1 RNAi embryos. 

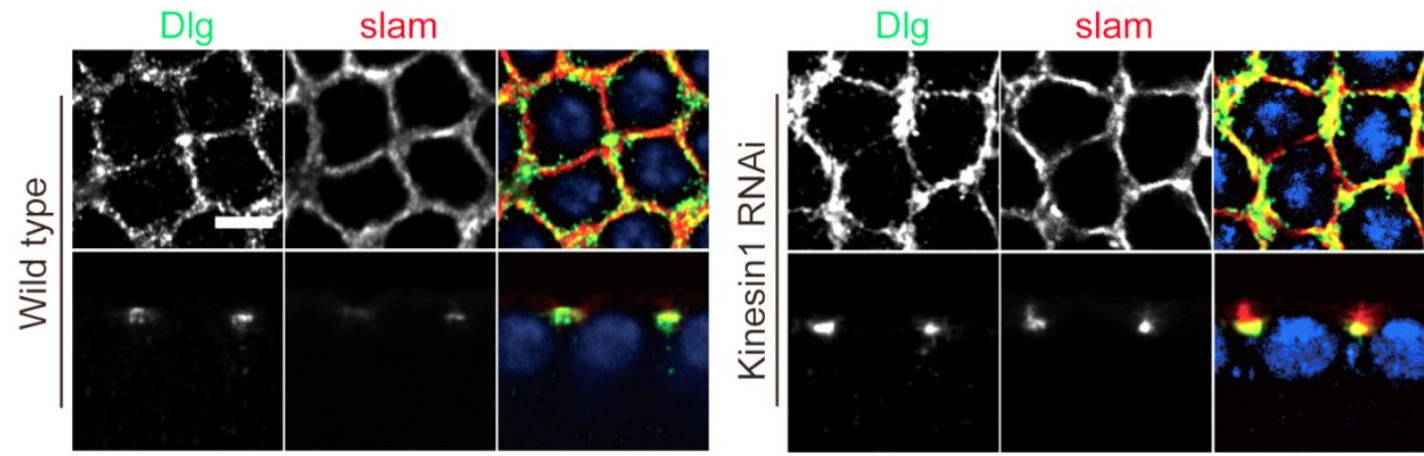

Figure 25 The intercap domain is not affected in Kinesin-1 RNAi embryos.

The localization of Slam (red) and Dlg (green) in wild type and Kinesin-1 RNAi embryos. Slam and Dlg accumulate to the intercap domain during the syncytial interphase of wild type and Kinesin-1 RNAi embryos. Nuclei are stained with DAPI (blue). Scale bar: $5 \mu \mathrm{m}$.

The cap domain was detected in both wild type and Kinesin-1 RNAi embryos via the phalloidin staining, as F-actin accumulated at the cap domain during the interphase of syncytial blastoderms. The result showed that, during the interphase of wild type and Kinesin-1 RANi syncytial blastoderms, F-actin formed isolated cap-like structures at the cap domain, the localization of F-actin at the cap domain in wild type and Kinesin-1 RNAi embryos was comparable (Figure 26). The result indicated that the cap domain existed in Kinesin-1 RNAi embryos, Kinesin-1 depletion did not affect the differetiation of cap and intercap domains.
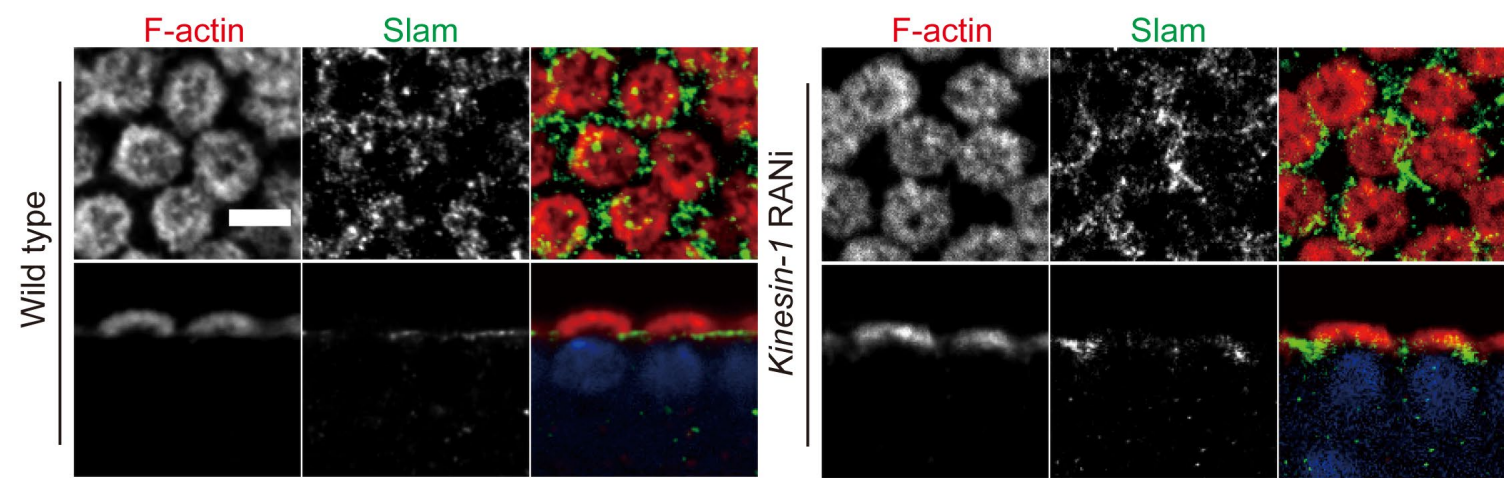

Figure 26 The differentiation of the cap domain is not influenced in Kinesin-1 RNAi syncytial blastoderms.

Localization of F-actin (red) and Slam (green) in wild type and Kinesin-1 RNAi embryos from surface view (first row) and section view (second row). F-actin accumulates at the cap domain, Slam localizes to the intercap domain. The cap domain and the intercap domain are exist in both wild type and Kinesin-1 RNAi embryos during the syncytial stage. Scale bar: $5 \mu \mathrm{m}$.

\subsection{The organization of F-actin is altered in Kinesin-1 depleted embryos}

The stainings of Slam and F-actin indicated that the cortical differentiation was not 
affected in Kinesin-1 RNAi embryos during the syncytial interphase. Cortical F-actin filaments are polarized, the organization of F-actin is crucial for apical contraction (Coravos and Martin, 2016). To get detailed information about whether Kinesin-1 depletion influences the F-actin organization, live images of Utrophin-GFP in wild type and Kinesin-1 RNAi embryos were performed. Utrophin is an F-actin binding protein (Spracklen et al., 2014; Winder et al., 1995). The onset of the interphase of cell cycle 13 was defined as 0 min. The result indicated the localizaiton of UtrophinGFP at different depths during syncytial and cellularization stages. In wild type embryos, Utrophin-GFP formed caps during the syncytial interphase ( $0 \mathrm{~min}$ ) and cellularization (14 $\mathrm{min}$ ), and Utrophin-GFP caps dismissed when embryos entered into mitosis (12 min). However, in the Kinesin-1 RNAi embryos, although UtrophinGFP formed cap structures in the syncytial interphase (0 min) and cellularization (14 min), separation of Utrophin-GFP caps in Kinesin-1 RNAi embryos was not as clear as in wild type embryos. Furthermore, the accumulation of Utrophin-GFP at the depth of $3 \mu \mathrm{m}$ in Kinesin-1 RNAi embryos was not as sharp as in wild type embryos (Figure 27).

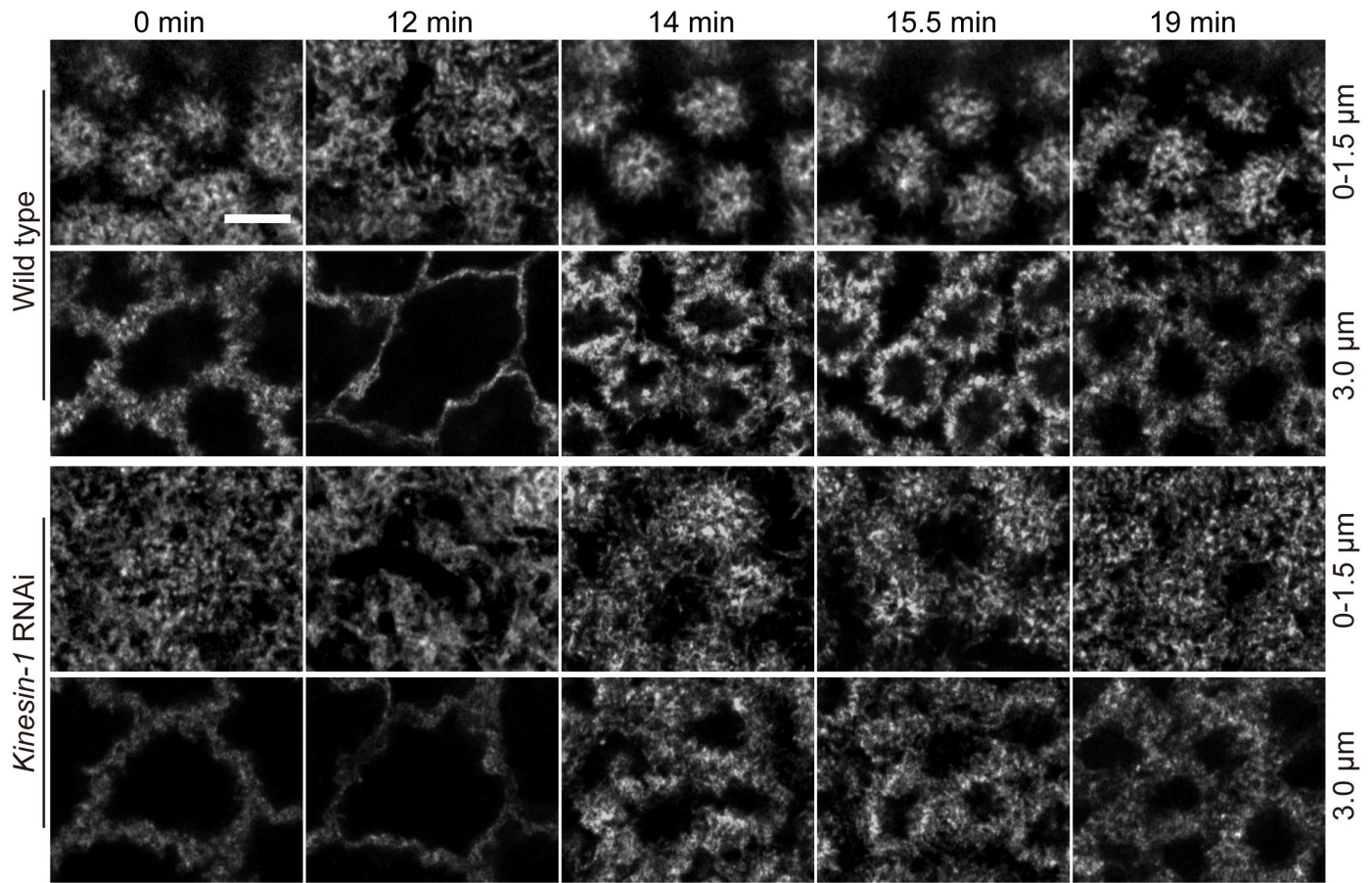

Figure 27 The localization of Utrophin-GFP is influenced in Kinesin-1 RNAi embryos.

The distribution of Utrophin-GFP in wild type and Kinesin-1 RNAi embryos during mitosis (0-12 min) and interphase (14-19 $\mathrm{min}$ ) at the indicated time points. During the interphase, Utrophin-GFP forms cap-like structures in wild type embryos and the separation between Utrophin-GFP caps is clear, while in Kinesin-1 RNAi embryos, the separation of Utrophin-GFP caps is affected, the distribution of Utrophin-GFP is not so restricted as in wild type embryos. Depth ranges indicate projection depths of Utrophin-GFP. Z-stack size of each step is $0.5 \mu \mathrm{m}$ and the time interval is $0.5 \mathrm{~min}$. Scale bar: $5 \mu \mathrm{m}$. 
Results from immunostainings of cortical components and live images of UtrophinGFP indicated that in Kinesin-1 RNAi embryos, although the F-actin cap structure existed, the organization of F-actin caps was affected.

F-actin filament is a polar structure, which has plus end and minus end. Plus ends of actin filaments display higher polymerization dynamics than minus ends (Pollard, 2016). Dynamics of actin filaments are regulated by a set of proteins. Arp2/3, a wellstudied actin nucleator, binds to the side of the existed actin filament, resulting in a branched actin network (Suarez et al., 2015b). Diaphanous (Dia), a member of Formin family proteins, binds to F-actin filament and enhances F-actin elongation at its plus end (Afshar et al., 2000; Higashida et al., 2004). Capping proteins includes Capping $\alpha(\mathrm{Cp \alpha})$ and Capping $\beta(\mathrm{Cp} \beta)$, bind to the plus end of F-actin filaments and block the polymerization of F-actin filaments (Bogdan et al., 2014; Edwards et al., 2014b; Suarez et al., 2015b; Svitkina and Borisy, 1999).

Live images of UtrophinGFP in Kinesin-1 RNAi embryos demonstrated that although F-actin caps existed during the interphase of syncytial blastoderms, the actin caps were changed in Kinesin-1 RNAi embryos compared to wild type embryos. To get detailed information about the defects of the F-actin caps organization in Kinesin-1 depleted embryos, immunostainings of Capping proteins were performed.

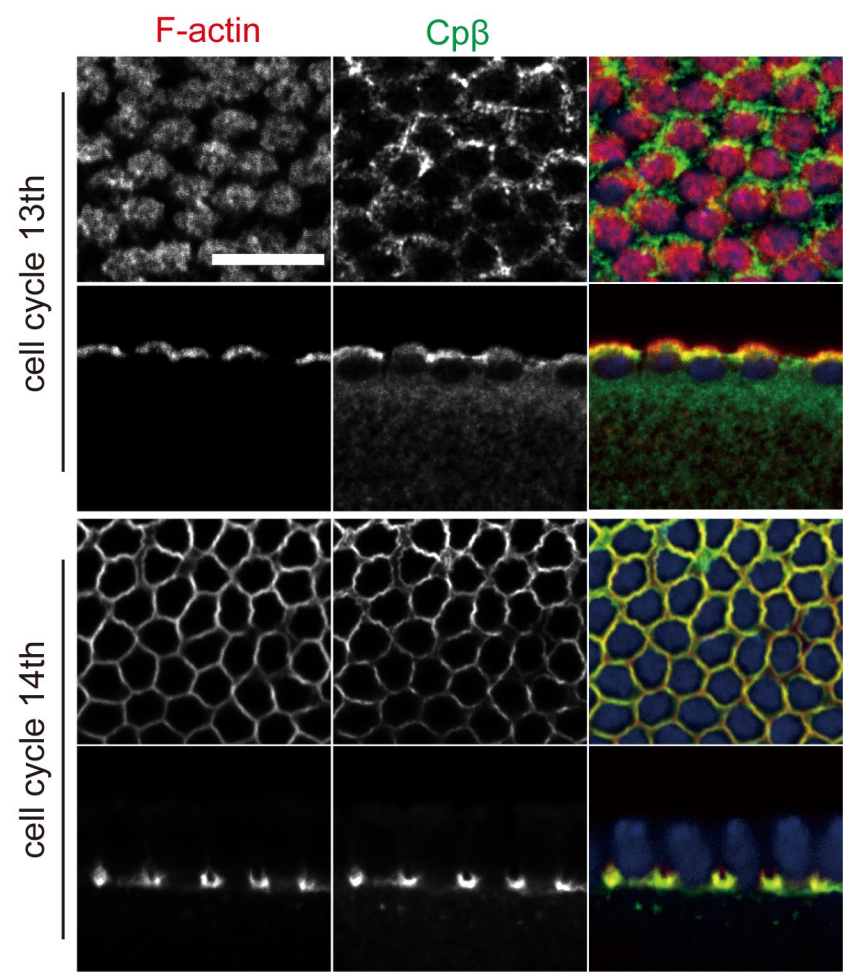

Figure 28 Localization of $C p \beta$ during syncytial and cellularization stages in wild type embryos. Localization of F-actin (red) and $\mathrm{Cp} \beta$ (green) in wild type embryos. The result shows that $\mathrm{Cp} \beta$ accumulates to the intercap domain during the interphase of cell cycle 13. During cellularization, both F-actin and $C p \beta$ move to the basal domain. Nuclei are stained with DAPI. Scale bar: $10 \mu \mathrm{m}$. 
I first checked the localization of $\mathrm{Cp} \beta$ in wild type embryos (Figure 28). During the interphase of cell cycle 13, F-actin accumulated and formed caps on the cortex of embryos. $\mathrm{Cp} \beta$ localized to the cortex of embryos, especially enriched at the edge of F-actin caps. It indicated that during interphase, plus ends of F-actin filaments accumulated at the intercap domain. During cellularization, with nuclear elongation and membrane invagination, F-actin and $C p \beta$ migrated to the basal domain.

Immunostaining of $\mathrm{Cp} \beta$ during the syncytial interphase indicated that plus ends of Factin filaments were well organized at the intercap domain, it is worth to check the organization of F-actin filaments in Kinesin-1 RNAi embryos. To confirm whether the F-actin organization was affected in Kinesin-1 RNAi embryos, I stained Cpa in Histone2Av-GFP (referred to hereafter as His-GFP) embryos (Kanda et al., 1998) and Kinesin-1 RNAi embryos together in the same tube. His-GFP embryos were used as control, the nuclear GFP signal was used to distinguish wild type embryos from Kinesin-1 RNAi embryos.

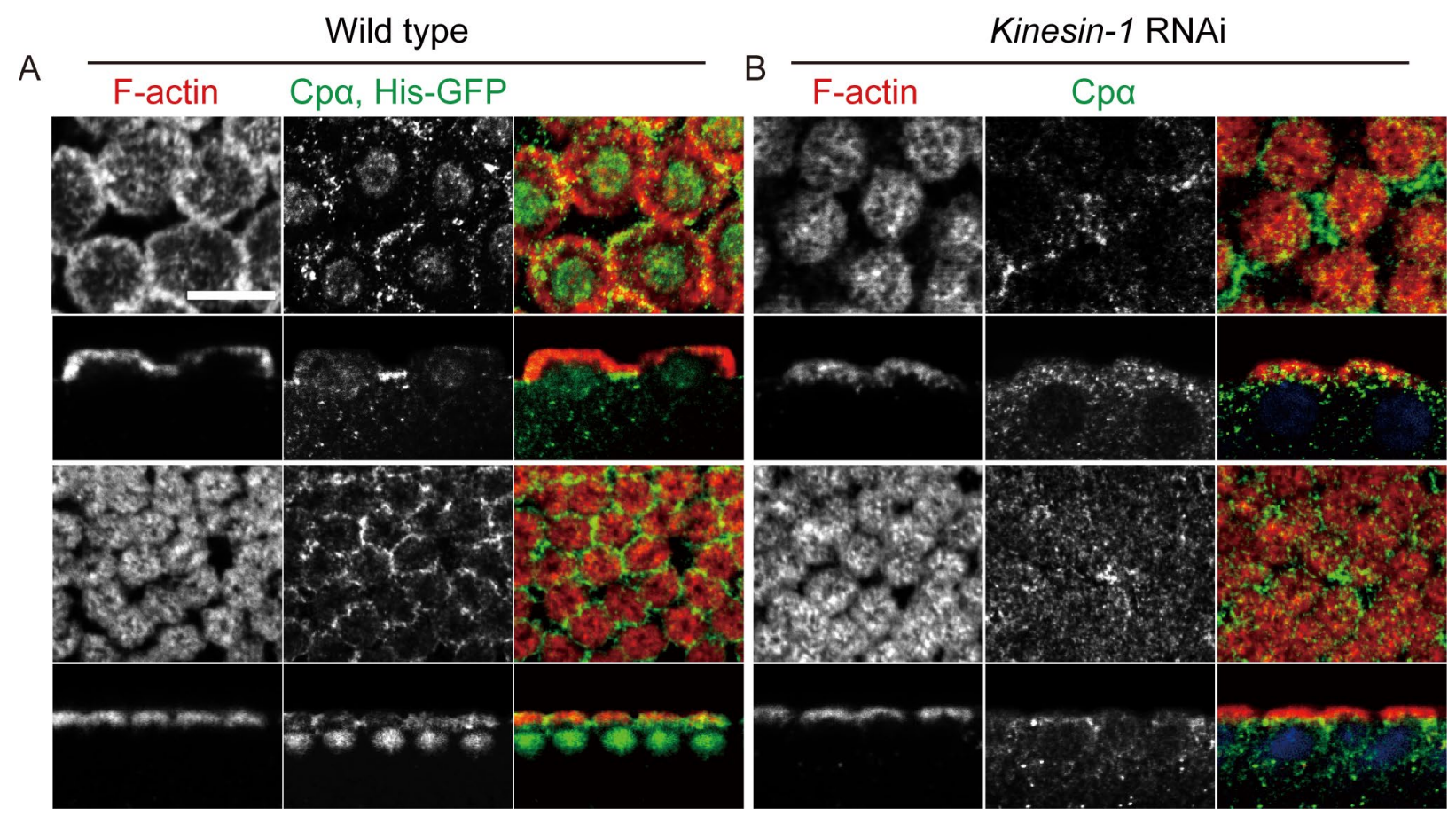

Figure 29 The localization of Cpa is affected in Kinesin-1 RNAi embryos.

Localization of F-actin (red) and Cpa (green) in wild type (A) and Kinesin-1 RNAi (B). Embryos are costaining in the same tube. The first row and the second row represent Cpa stainings in wild type and Kinesin-1 RNAi embryos in the interphase of cell cycle 11. The third row and the fourth row represent Cpa stainings in wild type and Kinesin-1 RNAi embryos in the interphase of cell cycle 13. Nuclei in wild type embryos are stained with GFP booster and nuclei in Kinesin-1 RNAi are stained with DAPI. Scale bar: $10 \mu \mathrm{m}$.

The result indicated that in wild type embryos, F-actin formed cap structures on the cortex of embryos and Cpa accumulated to the intercap domain during the 
interphase of syncytial blastoderms (both early and later cell cycles) (Figure 29A). While in Kinesin-1 RNAi embryos, during the interphase of syncytial blastoderms, although F-actin cap structures could be observed, the localization of Cpa was influenced, Cpa did not accumulate restrictly to the intercap domain in both early and later cell cycles (Figure 29B). The result proved that the distribution of F-actin filaments plus ends was affected in Kinesin-1 RNAi embryos.

I quantified the distribution of the Cpa fluorescent intensity from the edge of the caps in wild type and Kinesin-1 RNAi embryos during the interphase of cell cycle 13. The result showed that in wild type and Kinesin-1 RNAi embryos, relative fluorescent intensities of Cpa decreased from the edge to inner of cap. However, the declining rate in wild type was significantly faster than in Kinesin-1 RNAi, indicated that the relative amount of $\mathrm{Cpa}$ at the cap edge in wild type was significantly higher than that in Kinesin-1 RNAi (Figure 30A). The decline of Cpa fluorescent intensity in Kinesin-1 RNAi embryos may be due to bending of F-actin caps at their edges.
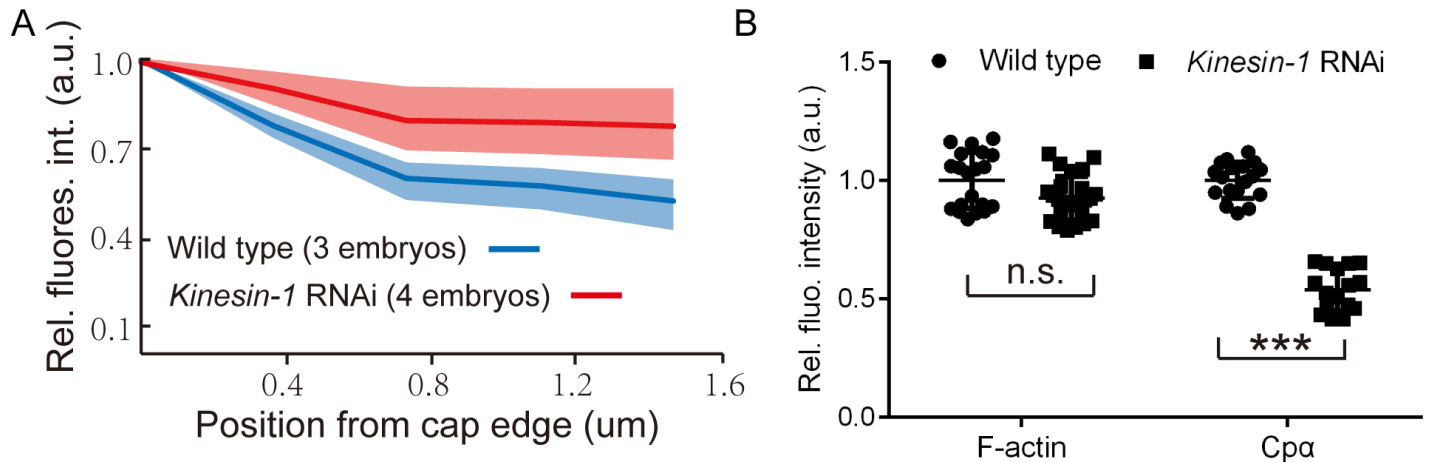

Figure 30 The Cpa distribution is disrupted in Kinesin-1 RNAi embryos.

(A) Relative fluorescent intensities of Cpa from the edge of caps in wild type (blue) and Kinesin-1 RNAi (red) embryos. Relative fluorescent intensities of Cpa in wild type (3 embryos) and Kinesin-1 RNAi (4 embryos) are measured, six measurements for each embryo. Means \pm SD are represented by transparent region along curves. (B) Relative fluorescent intensities of F-actin and Cpa at the cap domain during interphase. n.s., no significance; ${ }^{* * *}, p<0.001$. The P-value is calculated from the paired Student's t-test.

To better understand whether the Kinesin-1 depletion influenced the amount of Factin and $\mathrm{Cpa}$ at the cap region, measurements of the fluorescent intensity of F-actin and $\mathrm{Cpa}$ at the cap domain were performed (Figure 30B). The quantification showed that the amount of F-actin at the cap region was comparable between wild type and Kinesin-1 RNAi embryos. However, the amount of Cpa enriched at the cap domain was significantly higher in wild type than in Kinesin-1 depleted embryos. Quantifications of F-actin and Cpa proved that Kinesin-1 depletion not only 
influenced Cpa distribution but also affected the accumulation of Cpa at the cap region during the interphase.

To clarify the decrease of Cpa at the cap domain in Kinesin-1 RNAi was not due to the expression decline of $\mathrm{Cpa}$, the expression of Cpa was checked in wild type and Kinesin-1 RNAi embryos by western blot. The result showed there was no obvious change of Cpa expression in Kinesin-1 RNAi compared to wild type (Figure 31). Taken together, these results indicated that, the F-actin organization was affected in Kinesin-1 depleted embryos.

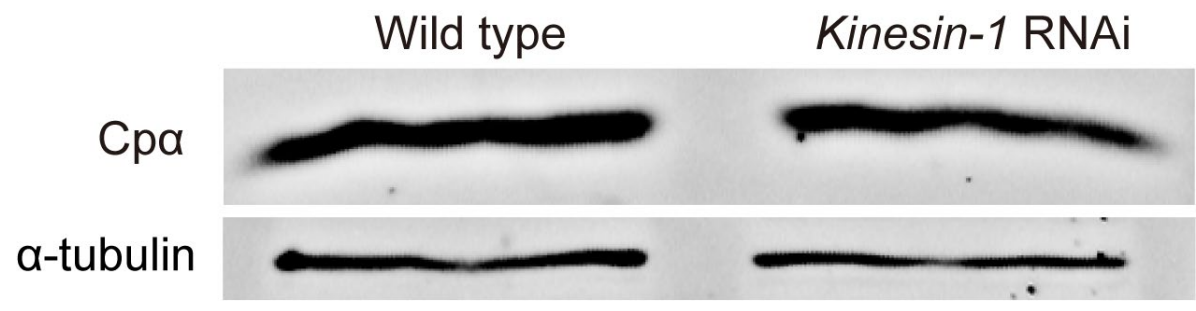

Figure 31 The Cpa expression is not affected in Kinesin-1 RNAi embryos.

The result shows the Cpa expression in wild type and Kinesin-1 RNAi embryos. The result indicates that the Cpa expression is comparable between wild type and Kinesin-1 RNAi. The expressions of $\alpha-$ tubulin are used as control.

3.6.1 Cpa-GFP clusters are mainly accumulated to the intercap domain during interphase

To better describe the organization of F-actin cap in early embryonic development, we generated an endogenous Capping a-GFP transgenic fly line by CRISPR/Cas9. The green fluorescent protein (GFP) coding sequence as well as stop codon were inserted into the $\mathrm{C}$ terminal of Capping a (Figure 32).

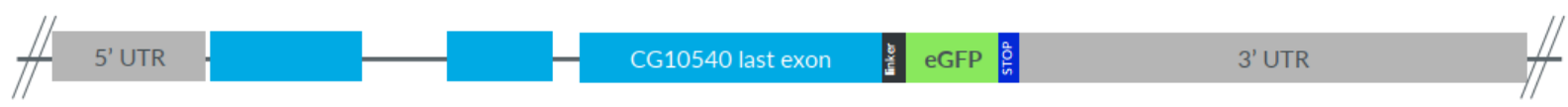

Figure 32 Schematic of Cpa-GFP.

The eGFP coding sequence and stop codon are inserted into the C-terminal of Cpa (CG10540) with CRISPR. This is introduced in materials and methods part.

It has been previously reported that $\mathrm{Cpa}$ clusters are observed in vitro experiment, the formation of $\mathrm{Cpa}$ clusters depends on the concentration of G-actin and Myosin II (Wollrab et al., 2019). Here I found that Cpa formed clusters in vivo by Cpa immunostainings in wild type and Cpa-GFP embryos (Figure 33). 


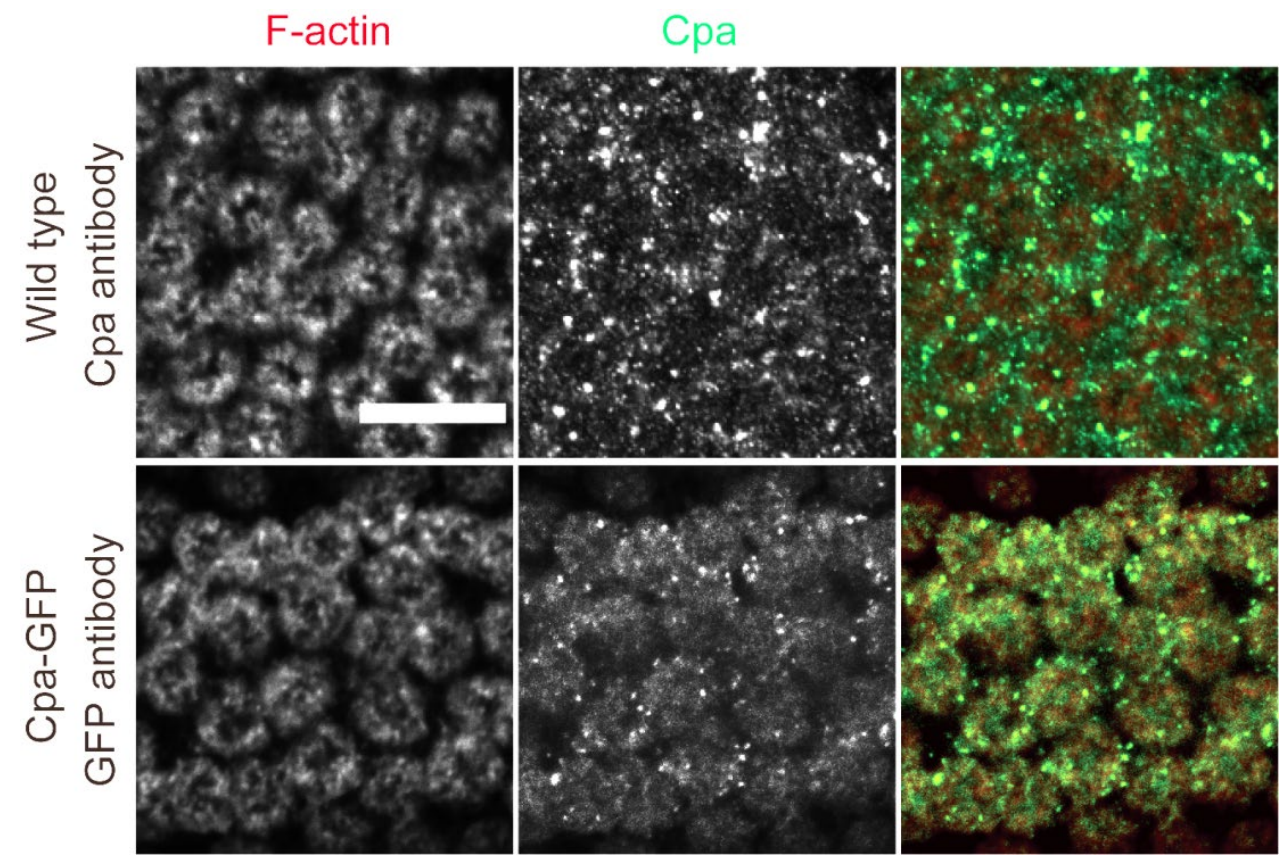

Figure 33 Cpa forms clusters in Drosophila early embryonic development.

The result shows the localization of F-actin (red) and Cpa (green) in wild type and Cpa-GFP embryos during the interphase. Cpa staining in wild type embryos with Cpa antibody, Cpa is stained with GFP antibody in Cpa-GFP embryos. Cpa clusters can be observed in both stainings. Cpa clusters localize to the edge of F-actin caps during the interphase. Scale bar: $10 \mu \mathrm{m}$.

Cpa-GFP clusters was also been found in live images of Cpa-GFP embryos. CpaGFP clusters were stable over several minutes. The projection of live images showed that Cpa-GFP clusters could be found at the intercap domain (Figure 34).

\section{Cpa-GFP}

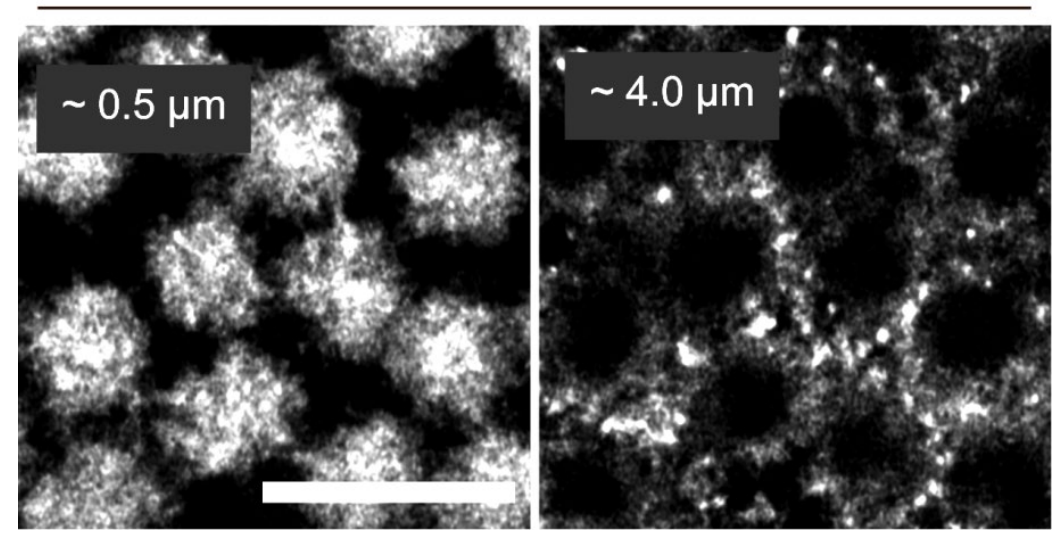

Figure 34 Cpa-GFP clusters localize to the cap and intercap domains during the interphase.

The result shows the distribution of Cpa-GFP clusters in different depths during interphase. Cpa-GFP clusters localize to the intercap domain during the interphase. Scale bar: $10 \mu \mathrm{m}$.

The immunostaining of $\mathrm{Cp \alpha}$ and $\mathrm{Cp} \beta$ indicated that plus ends of F-actin filaments localized to the intercap domain during the syncytial interphase and accumulated to the basal domain during cellularization. F-actin network is dynamic during mitosis, it is worth to check the localization of Cpa clusters in syncytial and cellularization 
stages.

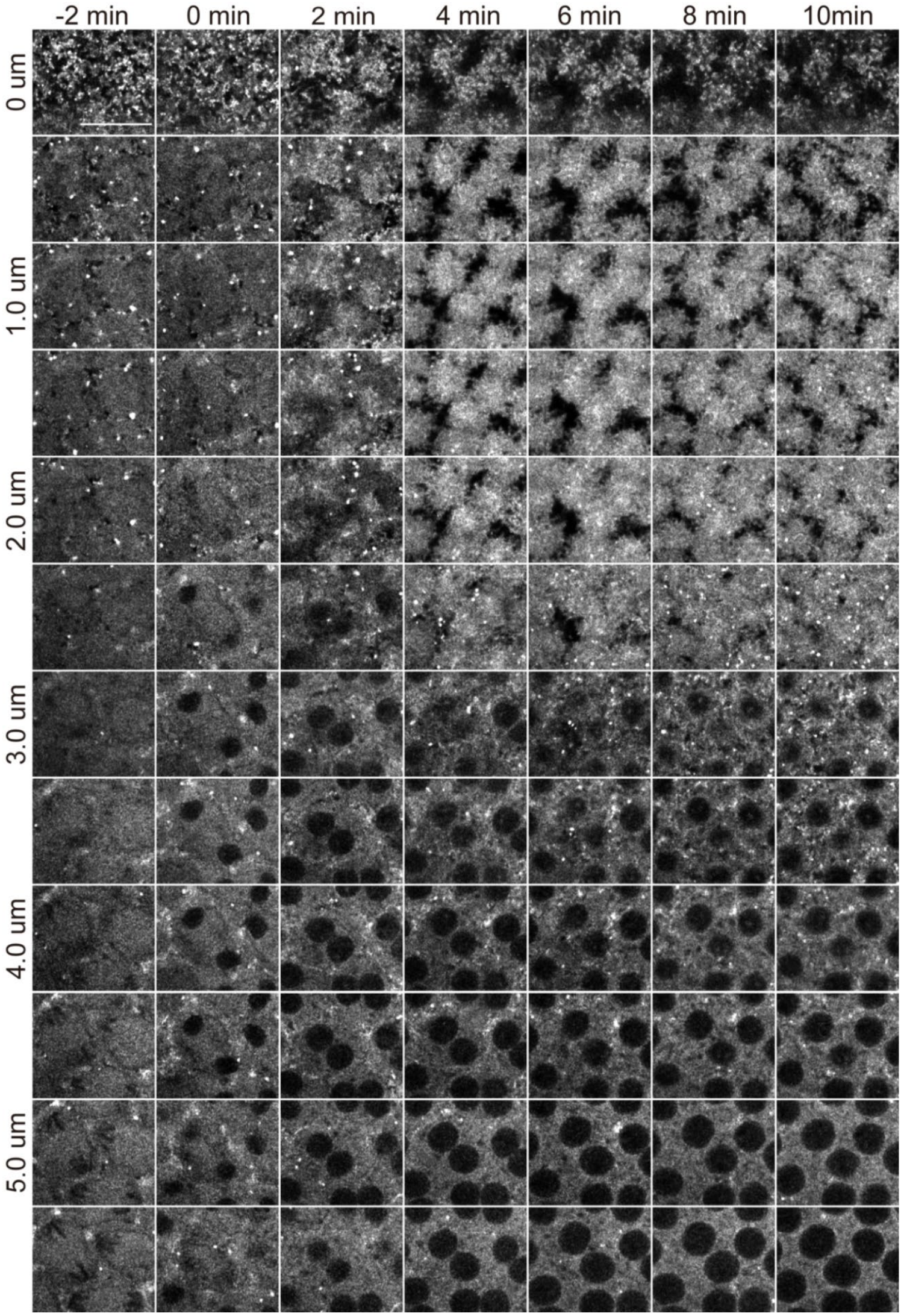

Figure 35 Cpa-GFP clusters move to intercap domain at the onset of cellularization.

The live images show the localization of Cpa-GFP at the indicated time points and depths during the mitosis of cell cycle 13 (-2-0 min) and at the onset of cellularization (2-12 min). Z-stack size of each step is $0.5 \mu \mathrm{m}$ and the time interval is $2 \mathrm{~min}$. Scale bar: $10 \mu \mathrm{m}$. 
To check the localization of Cpa clusters during cellularization, I utilized embryos expressing Cpa-GFP and Moesin-RFP (Figure 35). The time of the new membrane emergence was defined as $0 \mathrm{~min}$. The result showed that, Cpa-GFP clusters mainly localized to the embryo cortex during mitosis (-2 $\mathrm{min})$. When embryos came to cellularization (2 $\mathrm{min}$ ), Cpa-GFP clusters migrated to the intercap domain. Furthermore, Cpa-GFP clusters moved to the furrow canal during cellularization, Cpa-GFP clusters could be found at $4 \mu \mathrm{m}$ depth in embryos ( $8 \mathrm{~min}$ ). The live images indicated that the distribution of Cpa-GFP clusters was dynamic during cellularization.

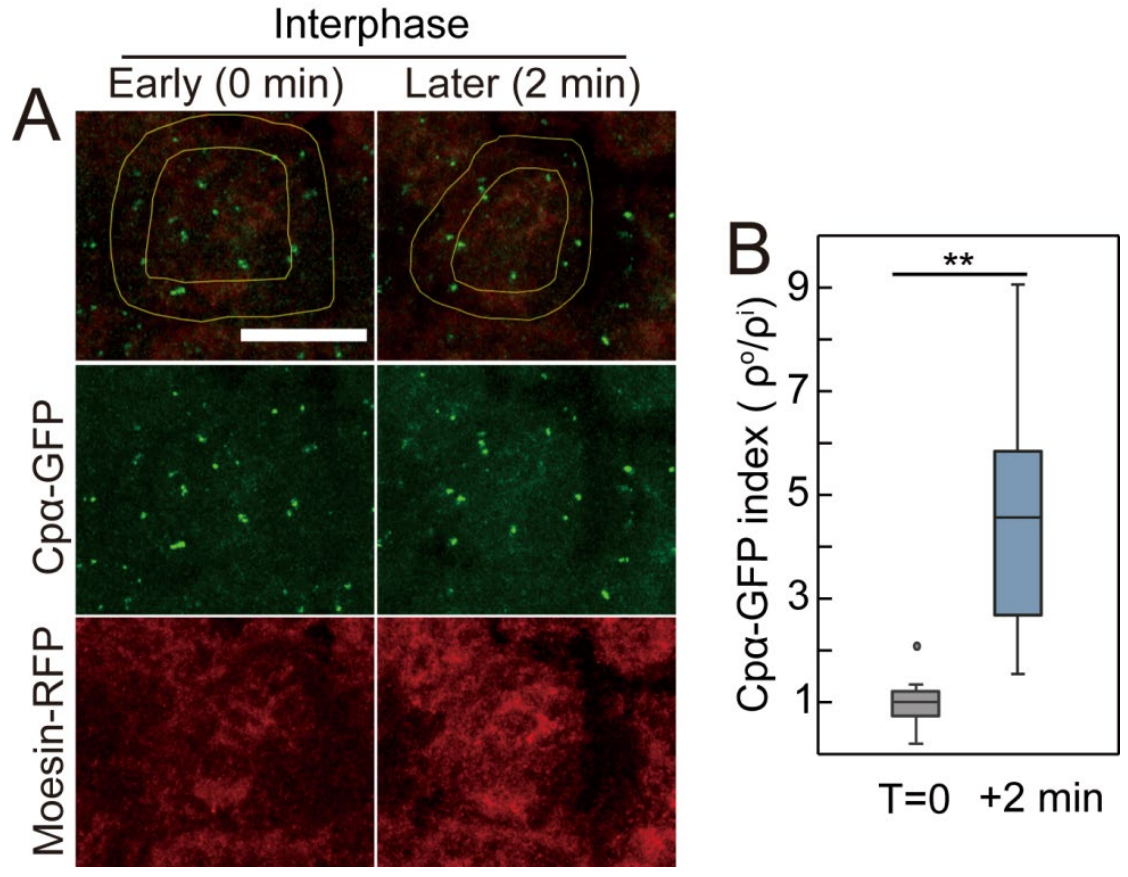

Figure 36 The Cpa-GFP distribution is dynamic during the interphase.

(A) The distribution of Cpa-GFP clusters at the onset of interphase (0 min) and during interphase (2 min). The cap region is marked with Moesin-RFP. (B) Measurement of Cpa-GFP clusters density at the inner $\left(\rho^{i}\right)$ and outer $\left(\rho^{\circ}\right)$ cap region in 2 min. $\rho=$ (the number of Cpa-GFP clusters)/(the area). Error bars indicate the minimum and maximum values, edges of boxes represent 25 th and 75 th percentile values respectively. ${ }^{* *}$ means $p<0.01$. The P-value is calculated from the paired Student's t-test. Scale bar: $10 \mu \mathrm{m}$.

The distribution of Cpa-GFP clusters was changed during the interphase. To clarify the organization of F-actin at cap and intercap domains during the interphase, numbers of Cpa-GFP clusters in 2 min during the interphase were measured and ratios $\left(\rho^{\%} / \rho^{i}\right)$ of Cpa-GFP clusters between the intercap domain $\left(\rho^{\circ}\right)$ and the cap domain $\left(\rho^{i}\right)$ were calculated. At the beginning of interphase $(0 \mathrm{~min})$, the ratio of CpaGFP clusters between the cap region and the edge of the cap was close to 1 (Figure 36), proved that Cpa-GFP clusters were equally distributed to the cap and intercap domains. However, during the interphase (2 $\mathrm{min}$ ), the ratio of Cpa-GFP clusters 
dramatically increased to about 4, indicated that the distribution of Cpa-GFP clusters changed, Cpa-GFP clusters are mainly accumulated to the intercap domain during the interphase. The quantification proved that the distribution of Cpa-GFP clusters was significantly changed during the interphase.

To figure out how the distribution of Cpa-GFP clusters was changed during the interphase, I recorded the movement of Cpa-GFP clusters every $2 \mathrm{~s}$ and marked positions of the cluster with different colors at different time points. The movement of Cpa-GFP cluster could be observed at the cap region, while the localization of Factin cap during this period was quite stable (Figure 37). This result indicated that Cpa-GFP clusters were quite dynamic during the interphase.

To clarify whether the movement of Cpa-GFP clusters resulted in the reorganization of Cpa-GFP clusters to the intercap domain, the direction of Cpa-GFP clusters movement was measured by recording the coordinates of Cpa-GFP clusters at different time points. Since the movement of cap domain during the interpahse, the coordinate of the cap center was changed. To solve this, for the same Cpa-GFP cluster, I corrected coordinates of cap center at different time points to the same reference coordinate. To calculate the orientation of Cpa-GFP cluster movement, the position of Cpa-GFP cluster before and after movement during a period was linked by a line.

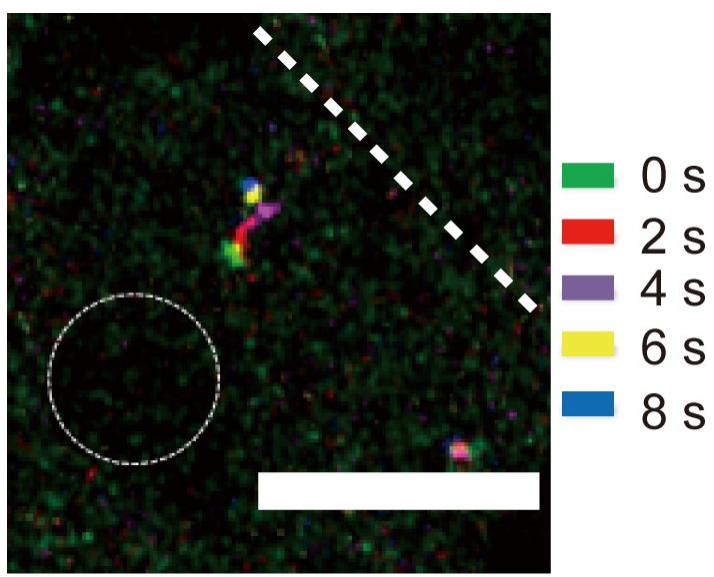

Figure 37 The movement of Cpa-GFP cluster during the interphase.

The Cpa-GFP cluster at different time points are labeled with different colors, the dashed circle indicates the nucleus, and the dashed line indicates the border of the cap. Scale bar: $5 \mu \mathrm{m}$.

For example, a line from reference coordinate to the coordinate of Cpa-GFP cluster at time $z\left(T_{z}\right)$ is drawn, towards to the coordinate of Cpa-GFP cluster at time $z\left(T_{z}\right)$; and then draw a line from the coordinate of Cpa-GFP cluster at time $T_{z}$ to the 
coordinate of Cpa-GFP cluster at time $z+1\left(T_{z+1}\right)$, towards to the coordinate of CpaGFP cluster at time $T_{z+1}$. The angle between these two lines is defined as $\alpha$, if the angle is smaller than 90 degree, it means that the Cpa-GFP cluster moves inward to the cap; if the angle is larger than 90 degree, it means that the Cpa-GFP cluster moves toward the edge of cap (Figure 38A).
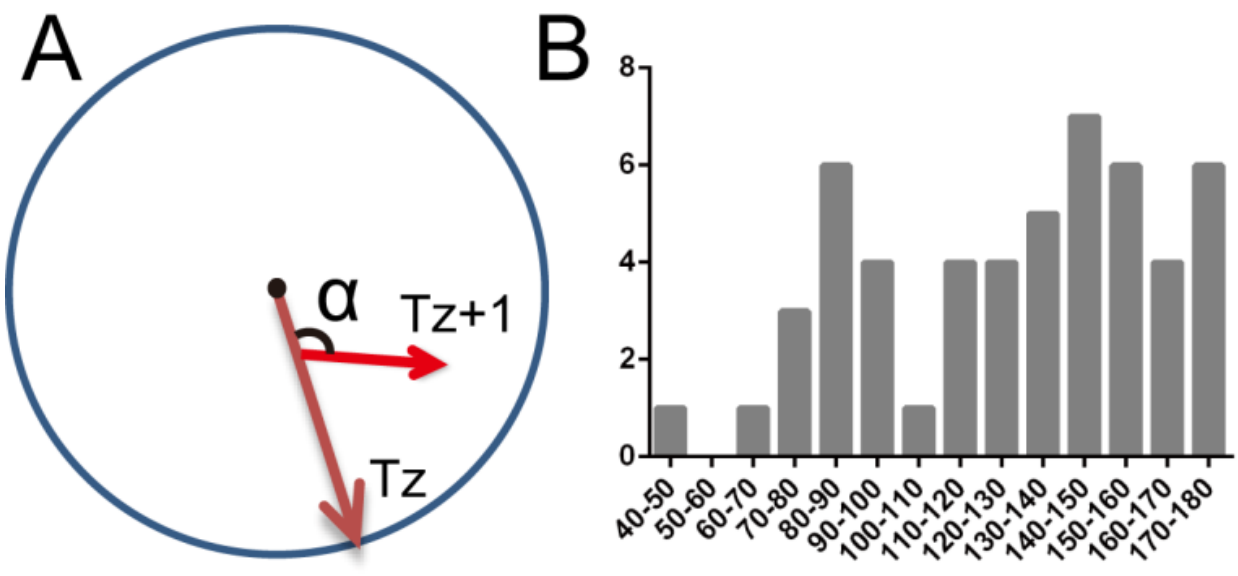

\section{Distribution of angle}

Figure 38 Quantification of Cpa-GFP clusters moving direction.

(A) Schematic of the direction of Cpa-GFP cluster movement from $T_{z}$ to $T_{z+1}$. (B) Angles of the CpaGFP clusters movement. 52 samples were measured.

Quantification showed that the majority of angles were larger than 90 degrees, indicated that in most case, Cpa-GFP clusters moved out to the cap edge (Figure 38B). The quantification proved that, Cpa-GFP clusters enriched at the edge of the cap during interphase was due to the outward movement of Cpa-GFP clusters. The velocity of Cpa-GFP clusters movement varied from $0.02 \mu \mathrm{m} / \mathrm{s}$ to $0.1 \mu \mathrm{m} / \mathrm{s}$, the average velocity of Cpa-GFP clusters movement was $0.069 \mu \mathrm{m} / \mathrm{s}$ (Figure 39).

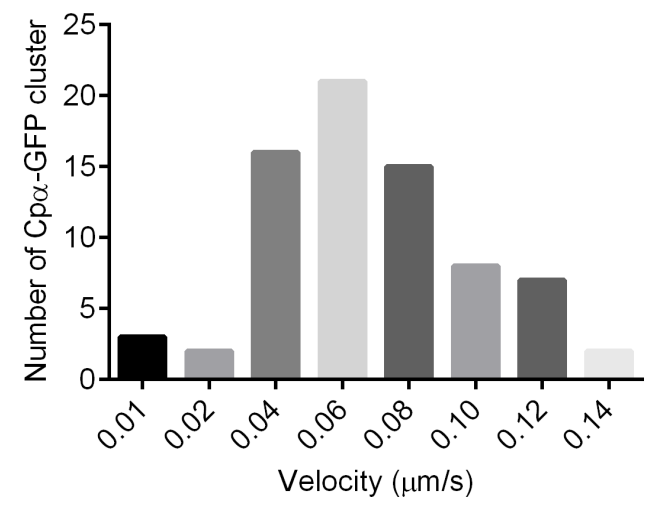

Figure 39 Velocity of Cpa-GFP clusters movement.

The result shows the velocity distribution of Cpa-GFP clusters in Cpa-GFP embryos.

The previous publication has shown that F-actin filaments form asters in the presence of Myosin II in vitro, with Cpa protein localizes to the center of F-actin 
filaments asters. The size of asters depends on the concentration of Myosin II and $\alpha-$ actinin. Furthermore, F-actin filaments asters display merging and splitting modes (Wollrab et al., 2019).

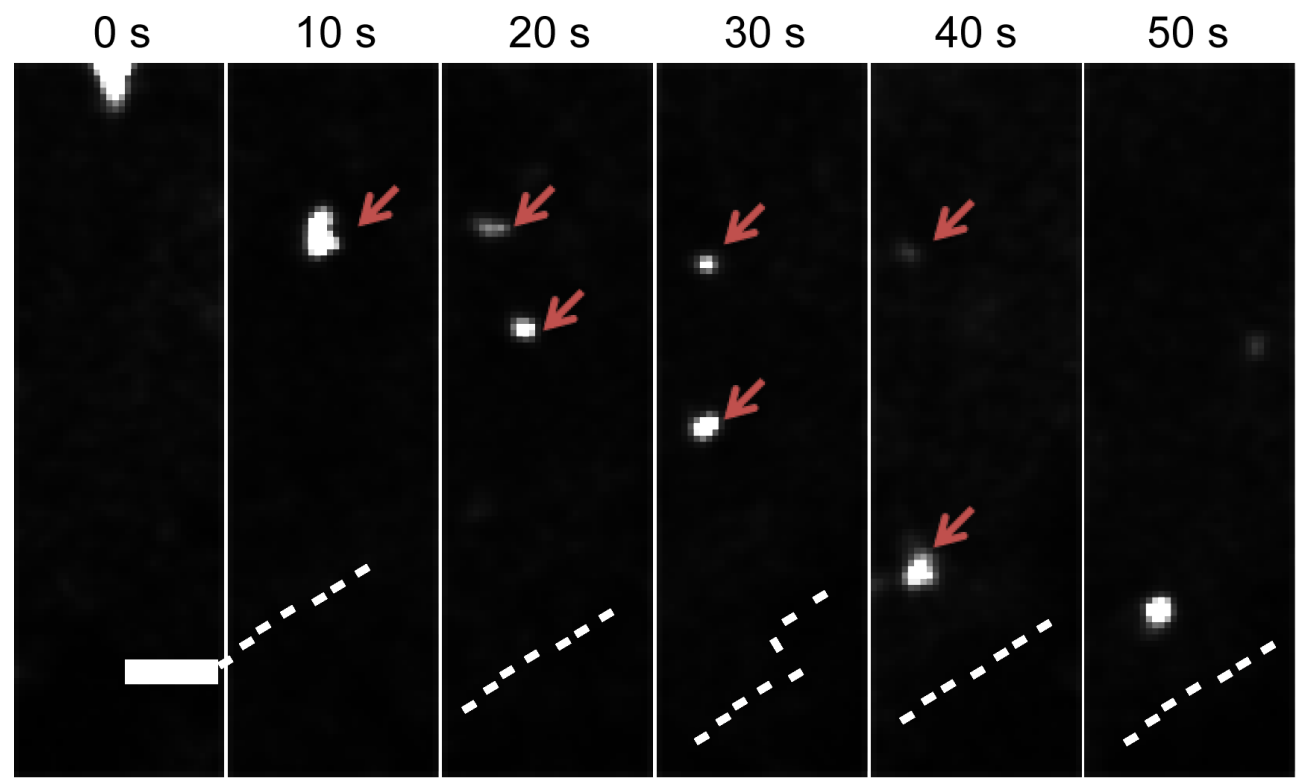

Figure $\mathbf{4 0}$ Splitting of the Cpa-GFP cluster.

The result shows the splitting of Cpa-GFP clusters in Cpa-GFP embryos at the indicated time points. Dashed lines indicate the edge of the cap domain, arrows indicate division and movement of the CpaGFP clusters. Scale bar: $2 \mu \mathrm{m}$.

In Cpa-GFP embryos, dynamics of Cpa-GFP clusters such as merging and splitting were also observed (Figure 40 and 41 respectively). The result showed that two isolated asters merged together in $80 \mathrm{~s}$ (Figure 40). The splitting of Cpa-GFP clusters was also recorded in live images (Figure 41).

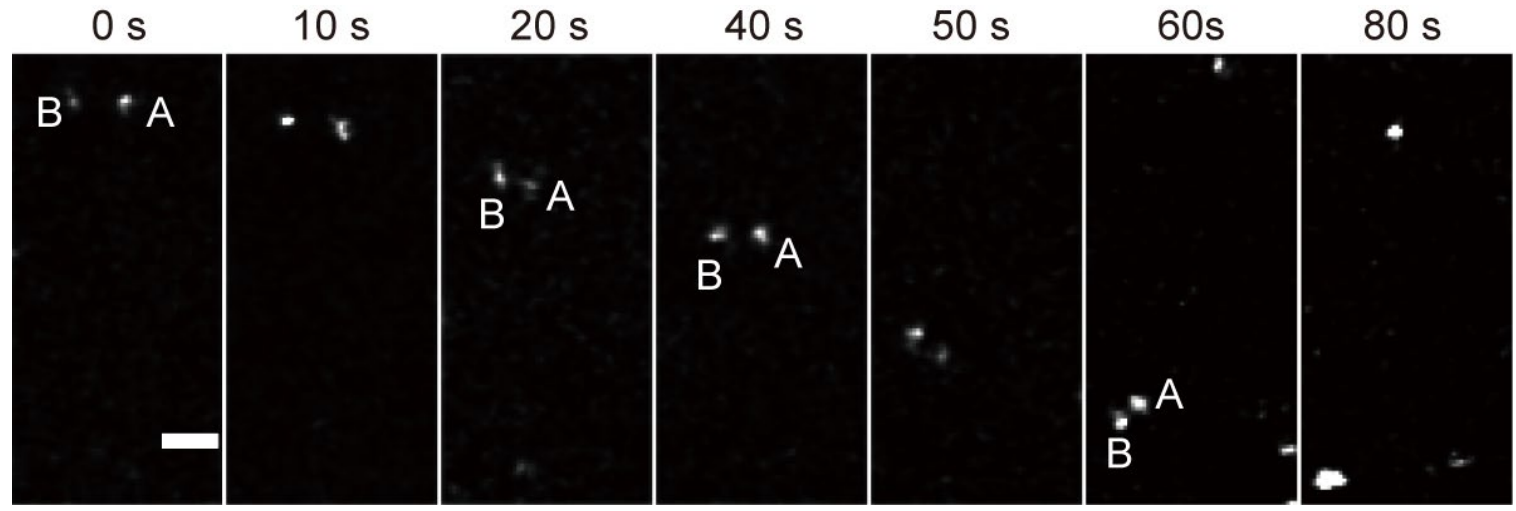

Figure 41 Merging of Cpa-GFP clusters.

The result indicates the merging of Cpa-GFP clusters. Life images show the movement of Cpa-GFP clusters and merging in $80 \mathrm{~s}$. A and B represent the two Cpa-GFP clusters at different time points. Scale bar: $2 \mu \mathrm{m}$. 
3.6.2 The localization of Cpa-GFP clusters is disrupted in Kinesin-1 RNAi embryos
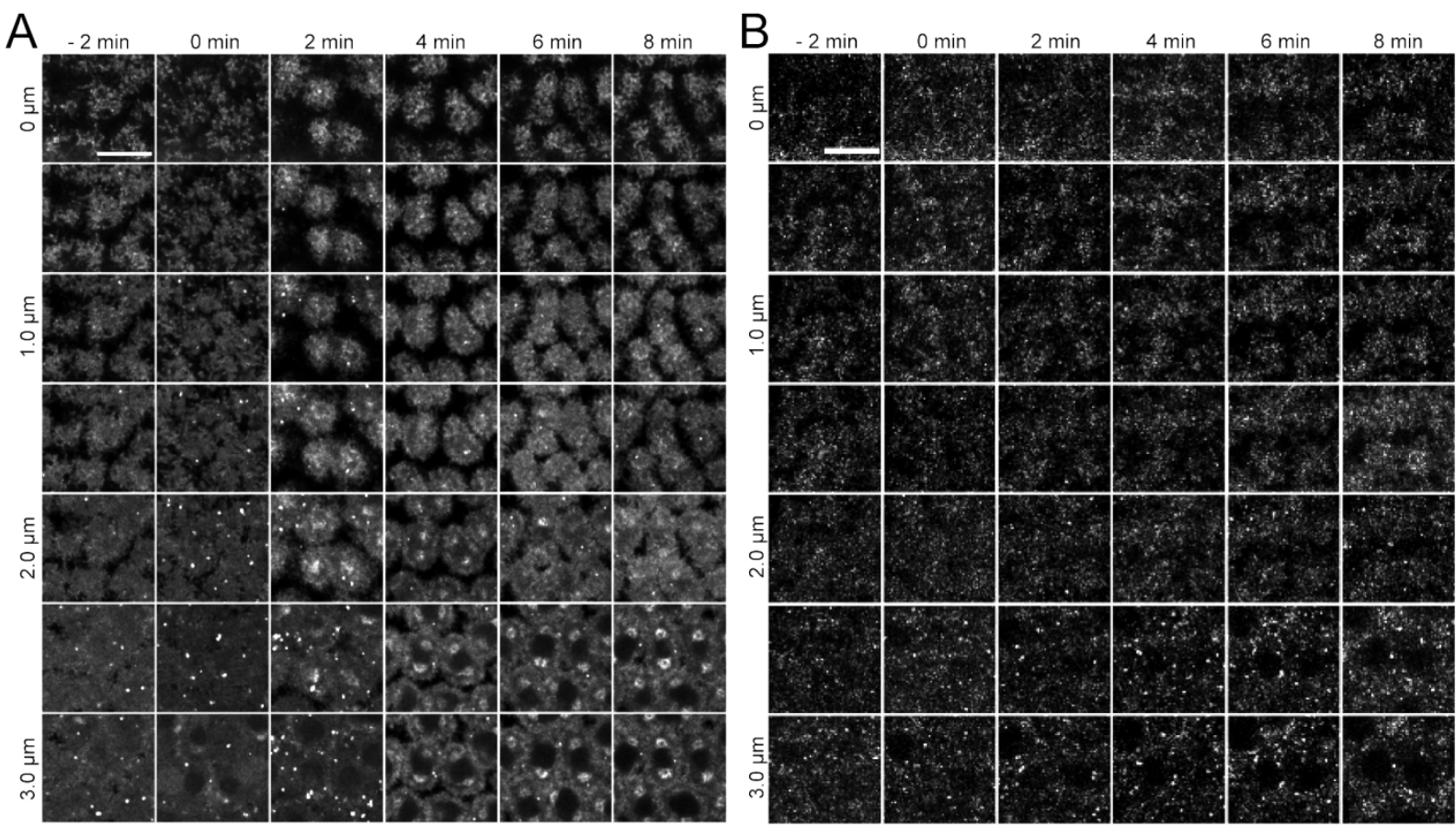

Figure 42 The distribution of Cpa-GFP clusters is affected by Kinesin-1 depletion.

Cpa-GFP clusters distribution in different depths of wild type (A) and Kinesin-1 depleted embryos (B) at the indicated time points during the syncytial stage. In Kinesin-1 RNAi embryos, Cpa-GFP clusters accumulate at the cap region during the interphase of the syncytial blastoderms. The time when nuclei formed was defined as $0 \mathrm{~min}$. Z-stack size of each step is $0.5 \mu \mathrm{m}$ and the time interval is $2 \mathrm{~min}$. Scale bar: $10 \mu \mathrm{m}$.

Previous results have shown that the Cpa distribution was affected in Kinesin-1 RNAi embryos. To further confirm that Kinesin-1 was involved in the Cpa distribution, I checked the distribution of Cpa-GFP clusters in wild type and Kinesin-1 RNAi embryos (Figure 42). In wild type syncytial blastoderms, Cpa-GFP clusters mainly localized to the intercap domain during the interphase. However, In Kinesin-1 RNAi embryos, Cpa-GFP clusters mainly accumulated at the cap domain during the interphase.

The quantification showed that, in wild type syncytial blastoderms, the Cpa-GFP clusters distribution changed in $2 \mathrm{~min}$, Cpa-GFP clusters mainly localized to the intercap domain (Figure 43). In Kinesin-1 RNAi embryos, I found the distribution of Cpa-GFP clusters was significantly different compared to wild type, Cpa-GFP clusters mainly localized to the cap domain during the interphase. The quantification indicated that Kinesin-1 was required for the Cpa-GFP clusters distribution. 


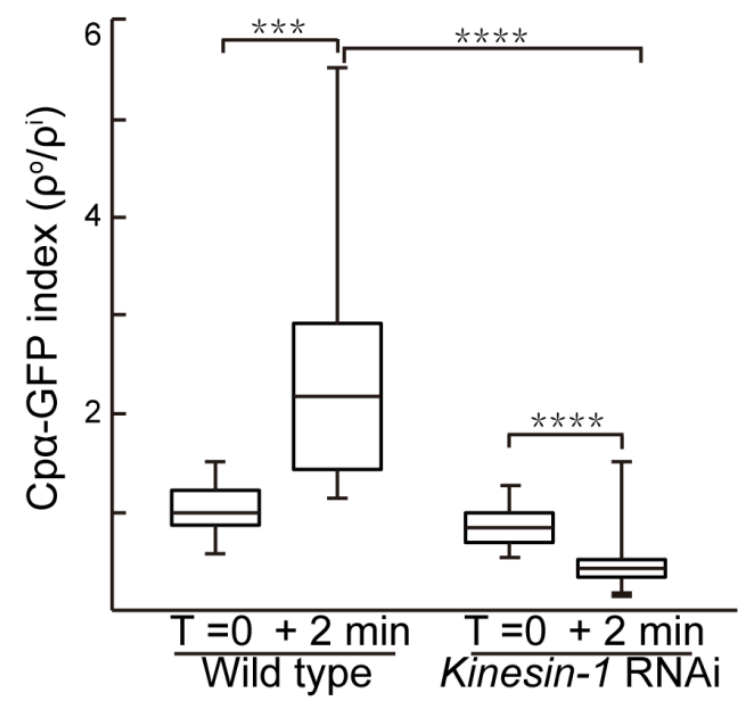

Figure 43 The distribution of Cpa-GFP clusters is affected in Kinesin-1 RNAi during the interphase of syncytial blastoderms.

Density of Cpa-GFP clusters at the cap $\left(\rho^{i}\right)$ and the intercap $\left(\rho^{\circ}\right)$ domains in 2 min during the interphase of wild type and Kinesin-1 RNAi embryos. The distribution of Cpa-GFP clusters in wild type and Kinesin-1 RNAi are quantified (both are five embryos, 3 measurements for each embryo). $\rho=$ (the number of Cpa-GFP clusters)/(the area). Error bars indicate the minimum and maximum values, edges of boxes represent 25 th and 75 th percentile values respectively. ${ }^{* * *}$ means $p<0.001,{ }^{* * * *}$ means $p<0.0001$. The P-value is calculated from the paired Student's t-test.

\subsection{Myosin II is required for the polarity of F-actin}

\subsubsection{Myosin II is mislocalized at the intercap domain in Kinesin-1 RNAi embryos}

Results from immunostaining of $\mathrm{Cpa}$ and live images of Cpa-GFP indicated that Kinesin-1 is required for the organization of F-actin cap, while how can Kinesin-1 regulate the organization of F-actin cap, also the link between Kinesin-1 and F-actin are not clear. From publications that we have, I assumed that Myosin II might be one candidate between Kinesin-1 and F-actin. As a motor protein, Myosin II binds and slides along F-actin filament to the plus end, the acto-myosin networks is particularly important for cell contraction and expansion (Svitkina, 2018; Svitkina and Borisy, 1999). Previous publication reported that Myosin II binds to and pulls F-actin filaments together based on its inherent motor activity (Clark et al., 2014). Furthermore, Myosin II drives polarity sorting processes and induces F-actin polar asters formation in vitro (Wollrab et al., 2019). Due to the interaction between F-actin and Myosin II, it is sensible to check the localization of Myosin II in Kinesin-1 depleted embryos, also whether the disruption of Myosin II influences the polarity of F-actin cap during the interphase. 
Spaghetti Squash (Sqh) encodes the Myosin II regulatory subunit in Drosophila (Karess et al., 1991). To check the whether Kinesin-1 depletion affected the localization of Myosin II, I utilized embryos expressing Spaghetti Squash (Sqh)-GFP to check Myosin II localization in Kinesin-1 RNAi embryos (Figure 44). The time when new nuclei formed was defined as $0 \mathrm{~min}$.

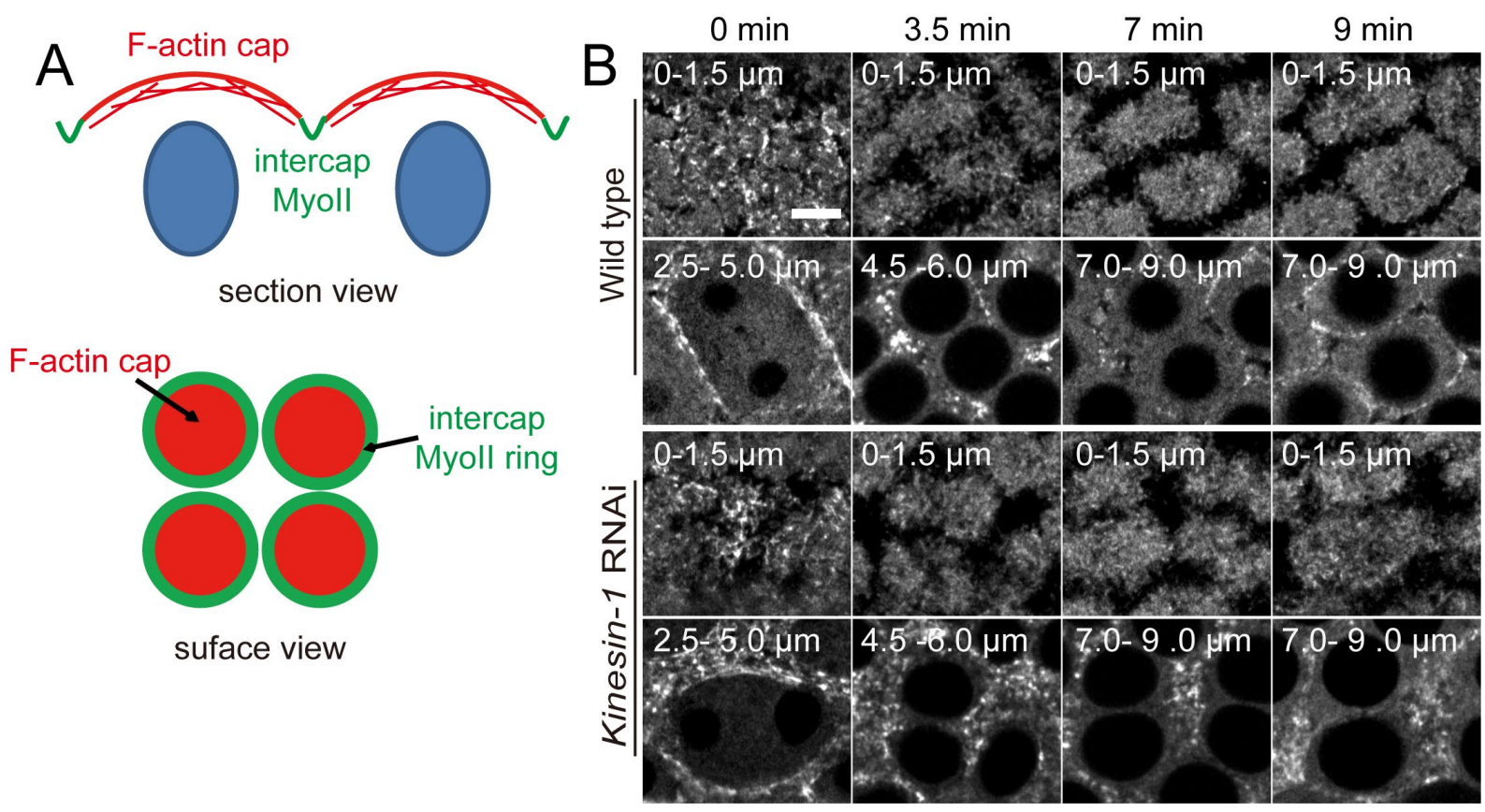

Figure 44 Sqh-GFP is mislocalized at the intercap region in Kinesin-1 RNAi syncytial blastoderms.

(A) Section view and surface view of Myosin II at the intercap domain during interphase. From the section view, Myosin II localizes to the intercap domain, F-actin localizes to the cap domain. From the surface view, Myosin II localizes to the intercap domain and forms rings around F-actin caps. (B) Images from time-lapse recordings of Sqh-GFP during the mitosis $(0 \mathrm{~min})$ and the interphase (3.5 to 9 min) of syncytial blastoderms in wild type (first and second rows) and Kinesin-1 RNAi (third and fourth rows) embryos in different depths. The ranges indicate projection depths of Sqh-GFP. Z-stack size of each step is $0.5 \mu \mathrm{m}$ and the time interval is $0.5 \mathrm{~min}$. Scale bar: $5 \mu \mathrm{m}$.

The scheme showed that during the interphase of syncytial blastoderms, F-actin accumulates at the cap domain and Myosin II localizes to the intercap domain and forms ring-like structures in wild type embryos (Figure 44A). The same localization of Myosin II could be found in live images of Sqh-GFP in wild type embryos. During the interphase of syncytial blastoderms, Myosin II not only accumulated at the cap domain but also enriched at the intercap domain. However, in Kinesin-1 depleted embryos, although Sqh-GFP could be found at the cap and intercap domains, the enrichment of Sqh-GFP at the intercap domain was not as restricted as in wild type embryos. This result indicated that the distribution of Myosin II was affected in Kinesin-1 RNAi embryos (Figure 44B). 

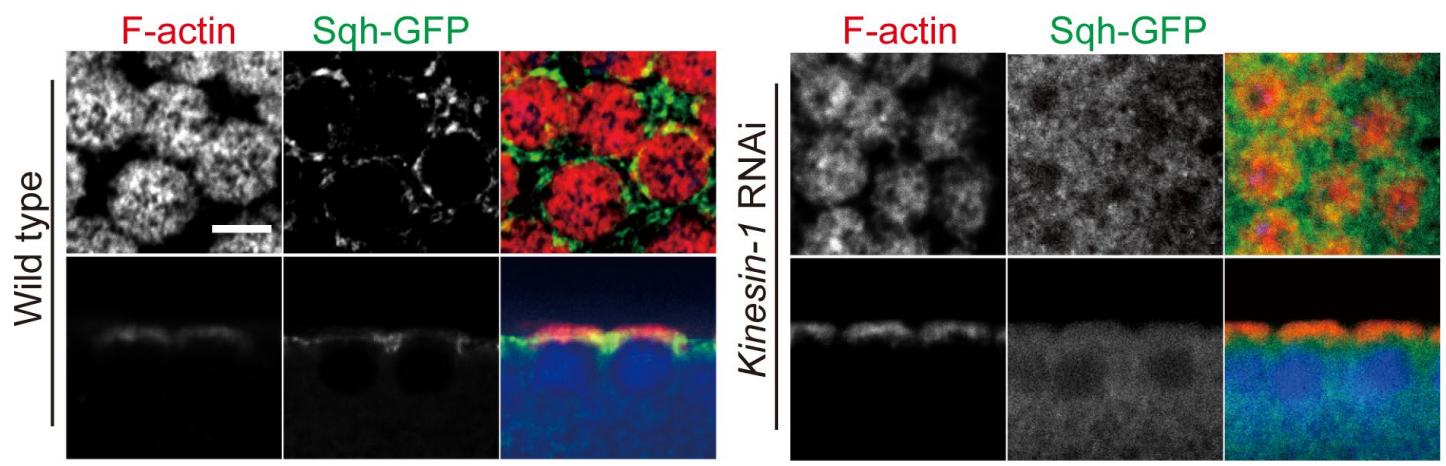

Figure 45 Sqh-GFP is mislocalized in Kinesin-1 RNAi embryos.

The result shows F-actin (red) and Sqh-GFP (green) localization from top view (first row) and section view (second row) in wild type and Kinesin-1 RNAi embryos during syncytial interphase, nuclei are stained with DAPI. Scale bar: $5 \mu \mathrm{m}$.

To get a better understanding of Myosin II localization in Kinesin-1 RNAi embryos, immunostaining of Myosin II was conducted in both wild type and Kinesin-1 RNAi embryos. The result showed that during the interphase, in wild type embryos, F-actin accumulated to the cap domain, Myosin II localized to the intercap domain in a ringlike pattern. While in Kinesin-1 RNAi embryos, although F-actin accumulated at the cap domain, Myosin II lost the enrichment at the intercap domain (Figure 45). To further confirm the localization of Myosin II in Kinesin-1 RNAi embryos, Zipper (Myosin II heavy chain) antibody was used (Fiehler and Wolff, 2007). The result showed that, the Myosin II accumulation at the intercap domain can be observed in wild type embryos, but the localization of Myosin II in Kinesin-1 RNAi embryos was affected (Figure 46). Taken together, live images and immunostainings of Myosin II proved that Kinesin-1 depletion influenced the localization of Myosin II at the intercap domain.

\subsubsection{The Cpa-GFP localization depends on Myosin II}

As Myosin II plays a vital role in F-actin asters formation in vitro and the Myosin II localization at the intercap domain was affected in Kinesin-1 RNAi embryos, it is reasonable to check whether Myosin II has function in F-actin organization. To confirm this, I checked the F-actin organization by immunostaining of Cpa after Y27632 injection. Y-27632 is a cell-permeable, highly potent and selective inhibitor of Rho-associated, coiled-coil containing protein kinase (ROCK). Y-27632 inhibits both ROCK1 and ROCK2 by competing with ATP for binding to the catalytic site (Davies et al., 2000; Ishizaki et al., 1996). 


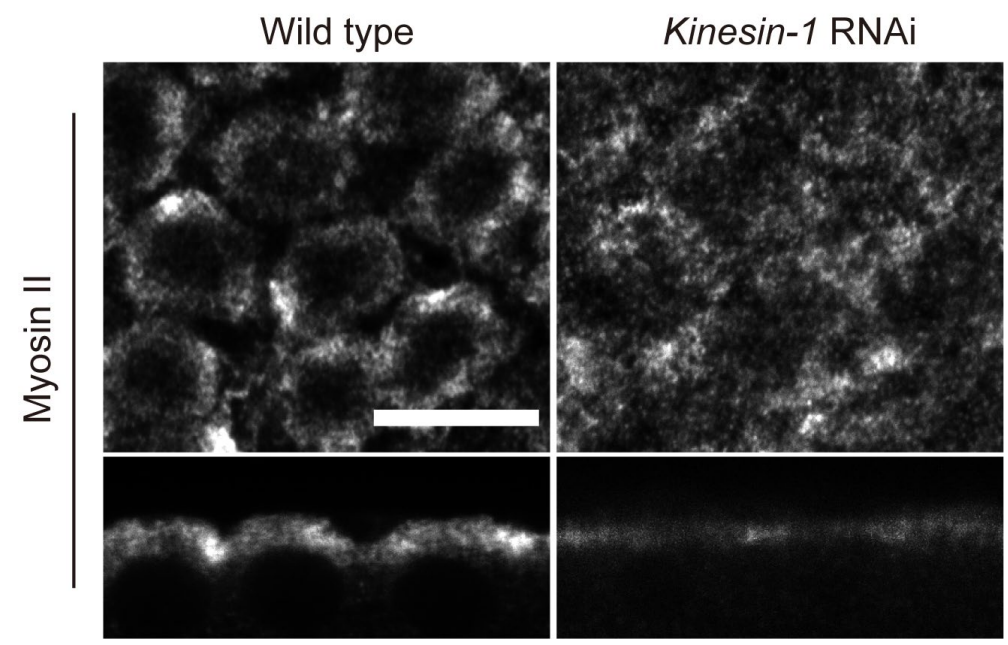

Figure 46 Myosin II is mislocalized in Kinesin-1 RNAi embryos.

The result shows the localization of Myosin II from surface view and section view during the interphase of the syncytial stage in wild type and Kinesin-1 RNAi embryos. Scale bar: $5 \mu \mathrm{m}$.

Myosin II localizes to the downstream of ROCK, I first checked the Myosin II inhibition by injecting Y-27632 into Sqh-GFP embryos. The result indicated that Y-27632 significantly decreased Myosin II activity at the intercap domain, the fluoresence of Sqh-GFP decreased (Figure 47).

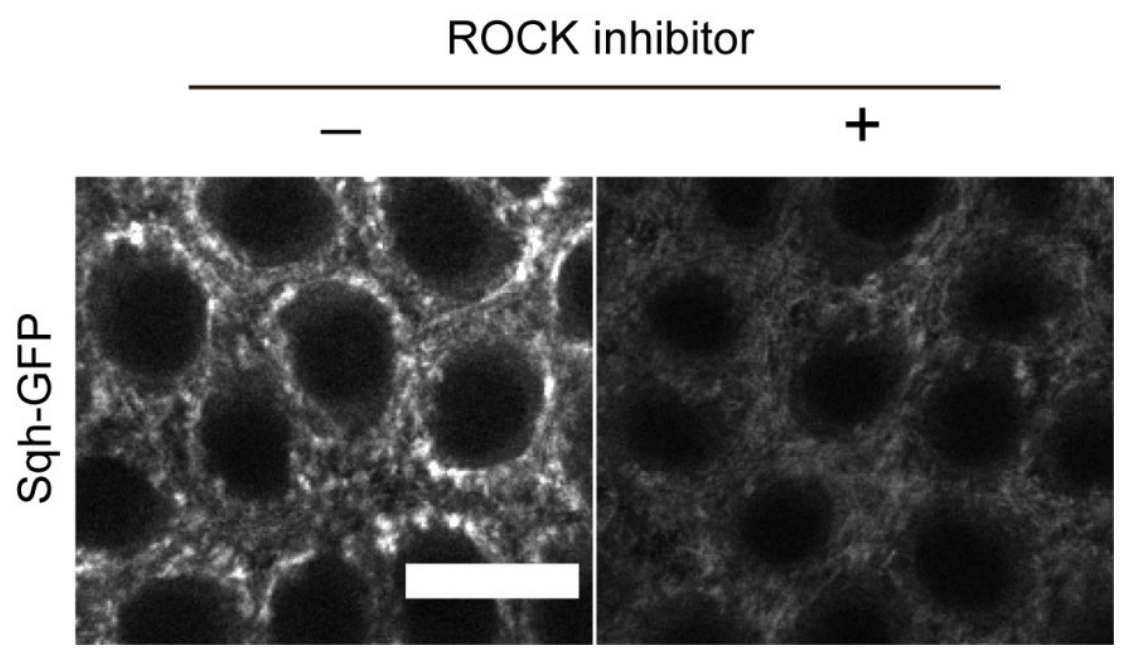

Figure 47 ROCK inhibitor decreases Myosin II activity at the intercap region.

The accumulation of Sqh-GFP at the intercap domain in embryos with or without ROCK inhibitor. (-) and $(+)$ indicate embryos without or with ROCK inhibitor injection. After ROCK inhibitor injection, the accumulation and activity of Myosin II at the intercap domain are decreased in Sqh-GFP embryos. Myosin II localizes to the downstream of Rho signaling. Myosin II is represented by Sqh-GFP. Scale bar: $10 \mu \mathrm{m}$.

Then I checked the Cpa localization at the intercap domain in embryos with Y-27632 $(10 \mathrm{mM})$ injection. The result showed that in wild type embryos, F-actin accumulated at the cap domain, and Cpa localized to the intercap domain during the interphase. However, after Y-27632 injection, although the localization of F-actin was not affected, 
the enrichment of $\mathrm{Cpa}$ at the intercap domain was not as sharp as in wild type embryos (Figure 48).

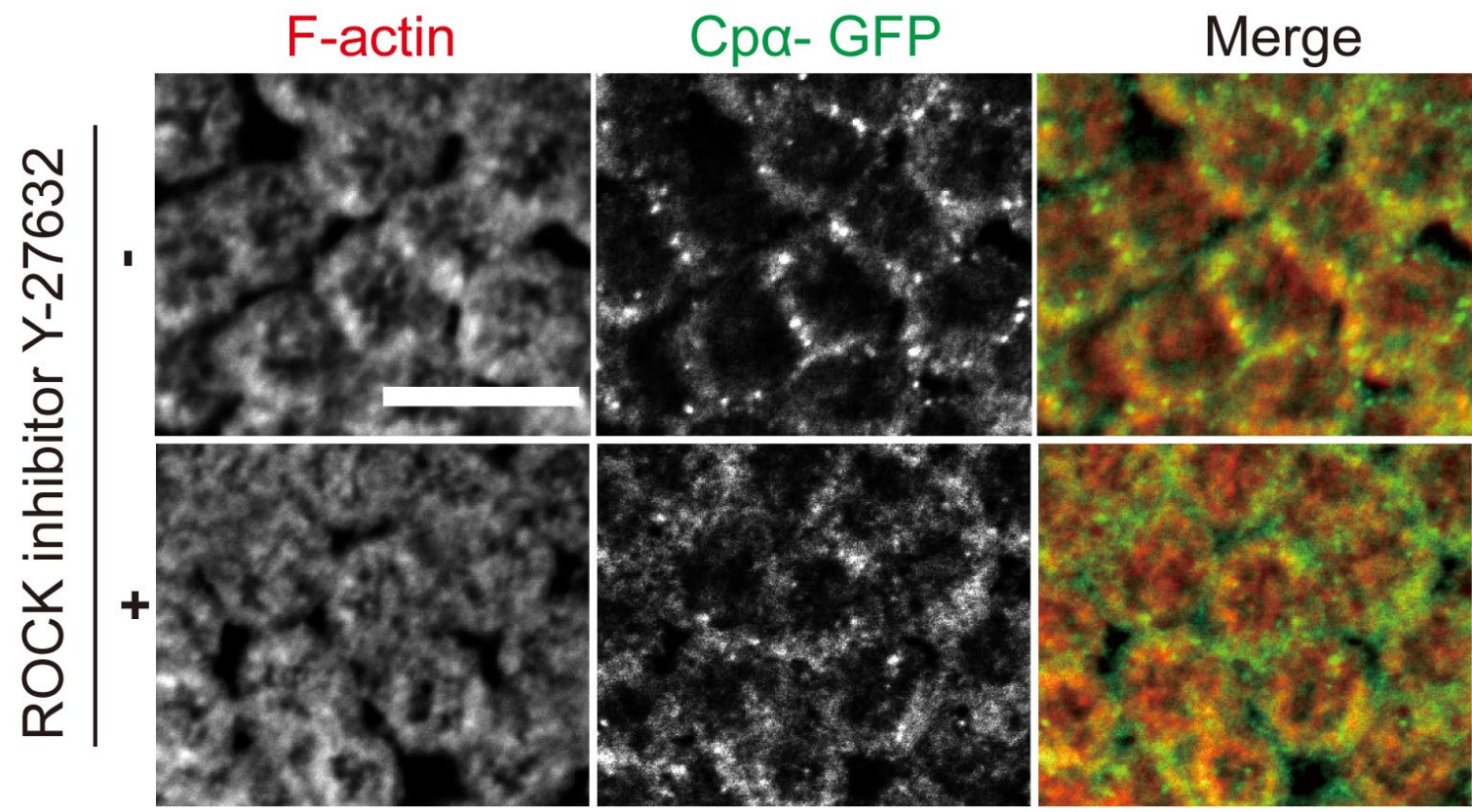

Figure 48 The Cpa-GFP distribution is affected by the Y-27632 injection.

The result shows F-actin (red) and Cpa-GFP (green) localization in Cpa-GFP embryos with (+) or without (-) ROCK inhibitor Y-27632 injection. The Cpa-GFP distribution in embryos with Y-27632 injection is affected compared to embryos without Y-27632 injection. The concentration of Y-27632 is $10 \mathrm{mM}$. Scale bar: $10 \mu \mathrm{m}$.

To get a better description about the function of Myosin II in the F-actin organization, the distribution Cpa fluorescent intensity from the cap edge in wild type and the $\mathrm{Y}$ 27632 injected embryos were measured. The result indicated that the Cpa fluorescent intensity decreased faster in wild type embryos compared to embryos with Y-27632 (Figure 49A). The distribution of F-actin did not change in embryos with Y-27632. Exponential decays of F-actin and Cpa fluorescent intensities were calculated. The result showed that the $\mathrm{Cpa}$ distribution rather than F-actin, was affected by Myosin II inhibition (Figure 49B). Results about Myosin II indicated that Kinesin-1 was required for the Myosin II localization and the distribution of Cpa depends on Myosin II activity.

Taken together, I proposed a simple scheme about the function of Myosin II in F-actin filaments organization in vitro and in vivo (Figure 50). In vitro, Myosin II catalyzes Factin filament asters formation and induces plus ends of F-actin filaments enriched at the center of aster. In vivo, Myosin II organizes F-actin filaments at the cap domain, plus ends of F-actin filaments enrich to the edge of cap during the interphase. 

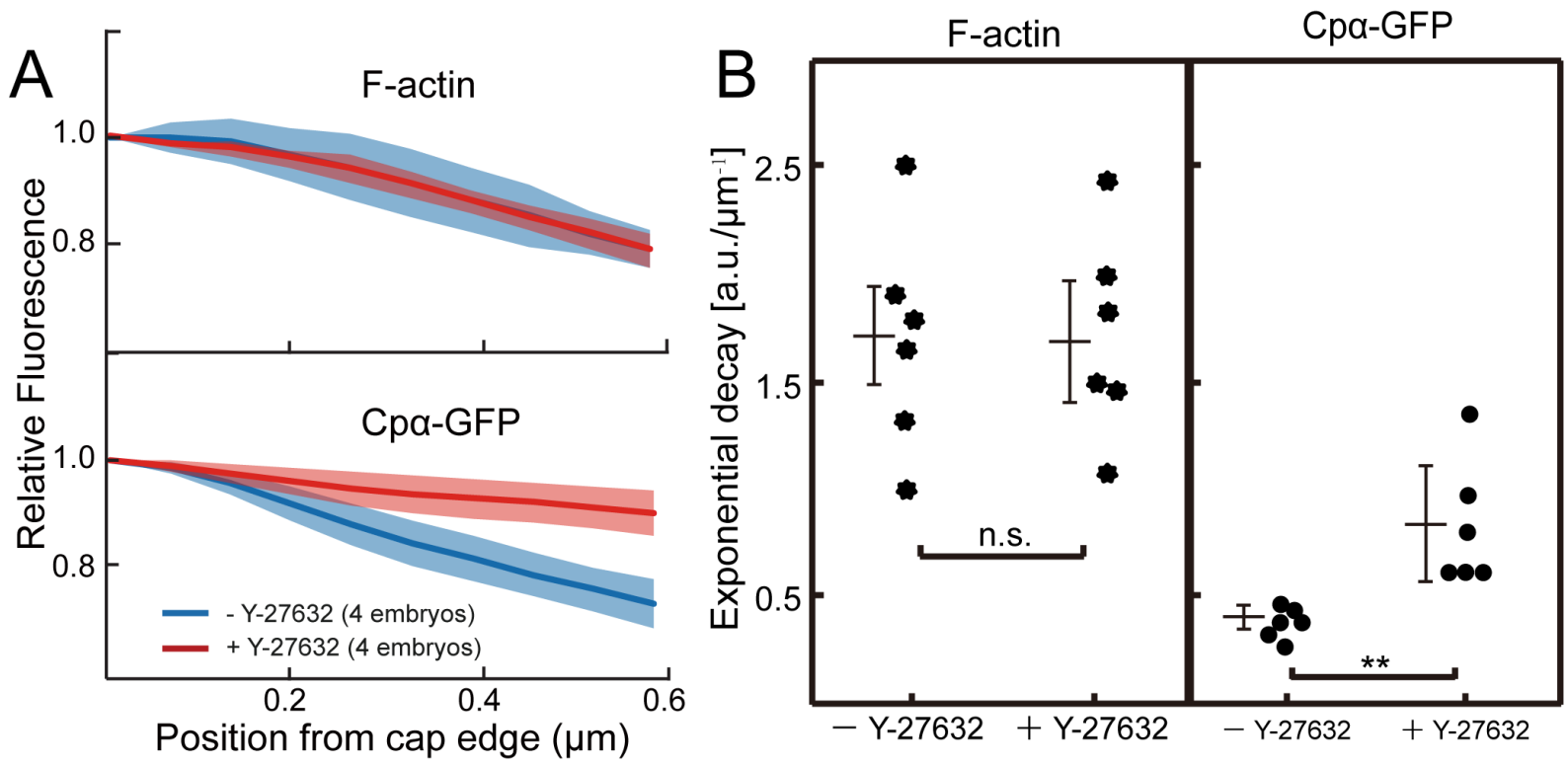

Figure 49 The Cpa-GFP distribution is influenced by Y-27632.

(A) Relative fluorescent intensities of F-actin and Cpa-GFP from the cap edge in embryos with or without Y-27632 injection. The quantification shows that in embryos with Y-27632 injection, although the F-actin localization does not alter, the accumulation of Cpa-GFP at the edge of the cap is disrupted. Means $\pm S D$ are represented by transparent region along curves. (B) Exponential decays of Cpa-GFP and F-actin with or without Y-27632. n.s., no significance; ${ }^{* *}, p<0.01$. The P-value is calculated from the paired Student's t-test.

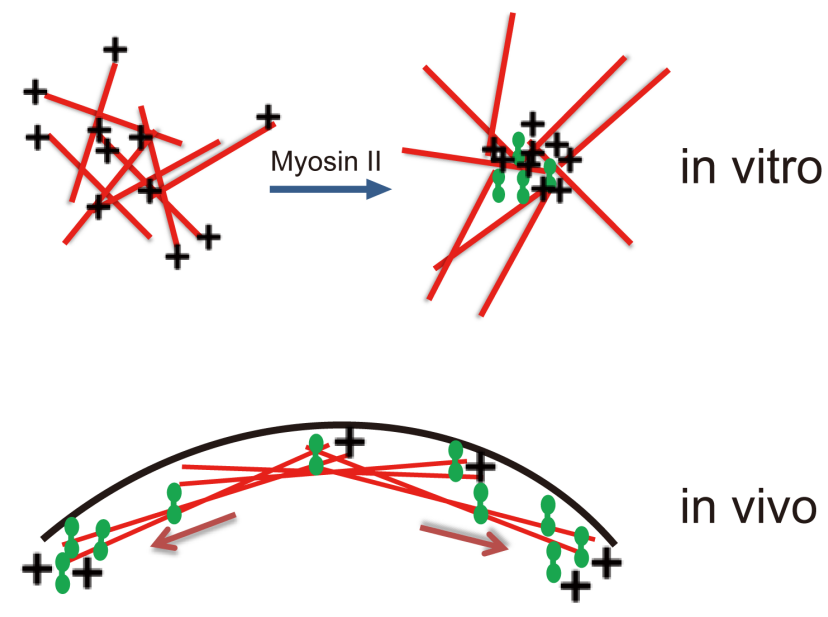

Figure 50 Schematic of Myosin II in F-actin organization in vitro and in vivo.

Red arrows indicate the directions of Myosin II movement in vivo, (+) means the plus end of F-actin filaments.

\subsection{The Cpa-GFP clusters distribution is affected in dia mutant}

Dia is a conserved protein, which has multiple functions in different cellular processes, such as cytokinesis, F-actin polymerization and microtubule dynamics (Bogdan et al., 2014). Previous publications reported that both Dia and Myosin II are downstream of 
ROCK signaling pathway (Afshar et al., 2000; Großhans et al., 2005; Lecuit et al., 2002). Results in this study about Myosin II indicated that Myosin II was affected in Kinesin-1 RNAi embryos, Myosin II was required for the F-actin organization. Thus, Kinesin-1 may influence the localization of Dia and disorder the F-actin organization.

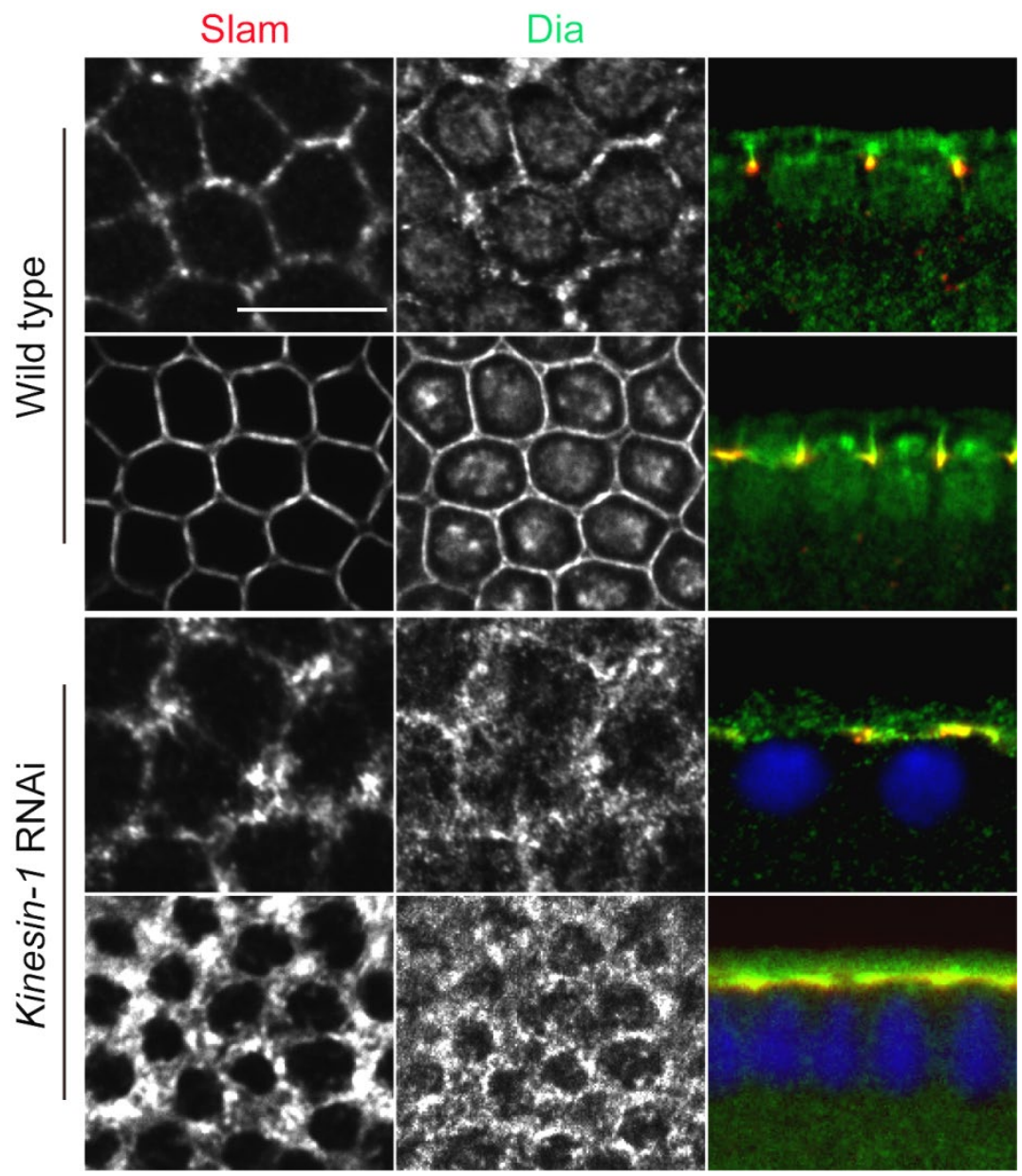

Figure 51 The localization of Dia is affected in Kinesin-1 depleted embryos.

The result shows Slam (red) and Dia (green) localization during syncytial and cellularization stages in His-GFP and Kinesin-1 RNAi embryos. His-GFP and Kinesin-1 RNAi embryos are stained in the same tube. Nuclei in wild type embryos are labeled with His-GFP, nuclei in Kinesin-1 RNAi embryos are stained with DAPI. Scale bar: $10 \mu \mathrm{m}$.

To confirm this, I checked the Dia localization in wild type and Kinesin-1 RNAi embryos. The result showed that, in wild type embryos, both Slam and Dia were localized to the intercap domain during the syncytial interphase and they migrated to the basal domain during cellularization. However, in Kinesin-1 depleted embryos, the localization of Dia was affected during syncytial and cellularization stages, Slam and Dia were stuck at the peripheral side of Kinesin-1 RNAi embryos (Figure 51). 
3.8.1 Dia is required for the Cpa localization in syncytial and cellularization stages
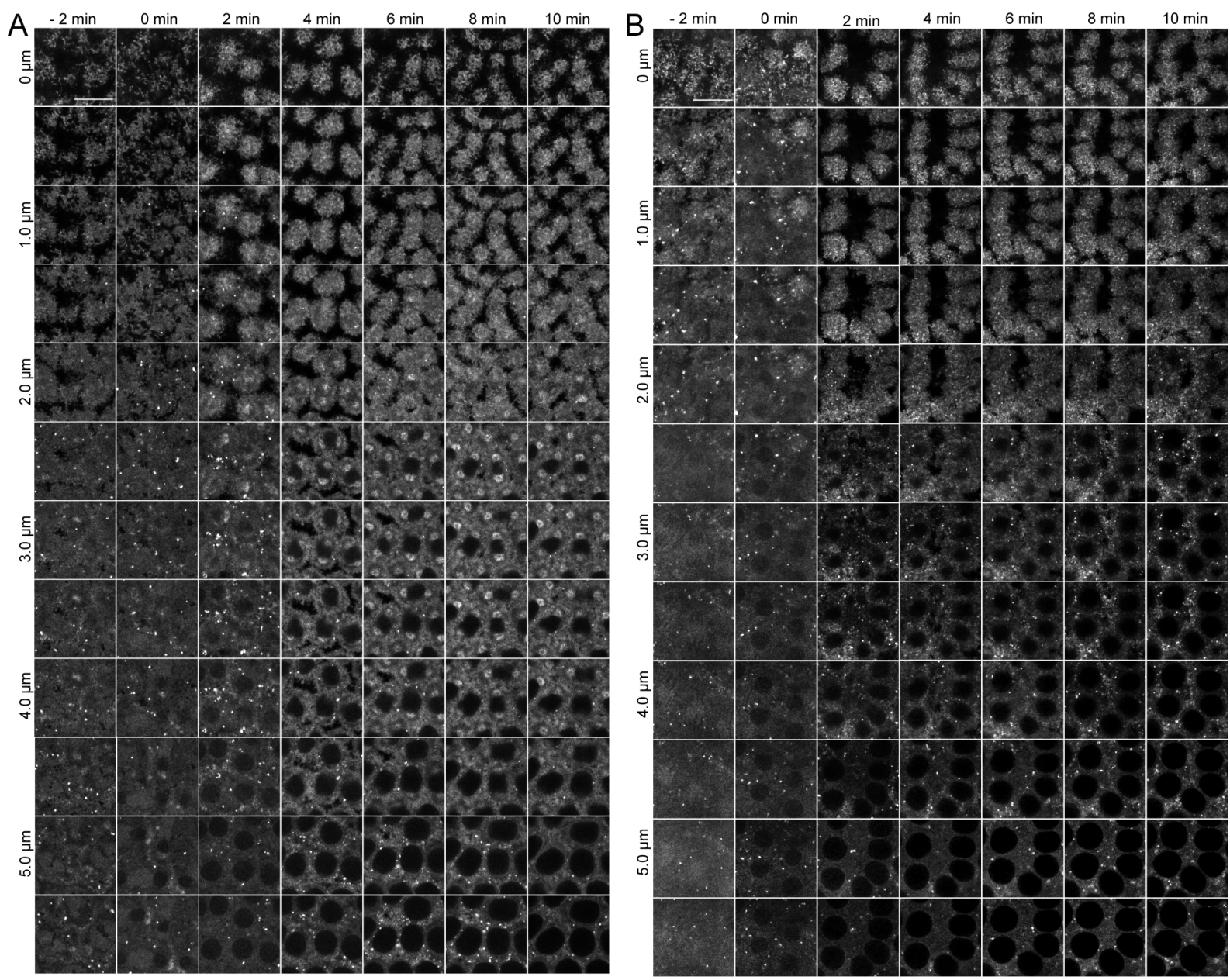

Figure 52 The Cpa-GFP clusters distribution is affected during the syncytial interphase of dia mutant.

The result shows the Cpa-GFP clusters distribution during mitosis (-2-0 min) and interphase (2-10 min) of syncytial blastoderms. (A) The distribution of Cpa-GFP clusters in the syncytial stage of wild type embryos. (B) The distribution of Cpa-GFP clusters in the syncytial stage of dia mutant embryos. The time when new nuclei are formed is defined as $0 \mathrm{~min}$, as the nuclear formation does not depend on dia. Z-stack size of each step is $0.5 \mu \mathrm{m}$ and the time interval is $2 \mathrm{~min}$. Scale bar: $10 \mu \mathrm{m}$.

Based on previous reports about functions of Dia and the result about the Dia localization in Kinesin-1 RNAi embryos, I assumed that Dia plays a role in the distribution of Cpa clusters. To test this hypothesis, the distribution of Cpa-GFP clusters in maternal dia mutant was investigated. The result showed the Cpa-GFP distribution in syncytial blastoderms of wild type (Figure 52A) and dia mutant (Figure 52B). The time when new nuclei formed was defined as $0 \mathrm{~min}$. In wild type, Cpa-GFP clusters mainly localized to the metaphase furrow (-2 min and $0 \mathrm{~min}$ ) during mitosis and localized to the intercap domain during interphase (2-10 min). However, in dia mutant embryos, although Cpa-GFP clusters were observed at the metaphase furrow 
during mitosis (-2 $\mathrm{min}$ and $0 \mathrm{~min}$ ), most of Cpa-GFP clusters were accumulated at the surface of dia mutant embryos. During the interphase (2-10 min), Cpa-GFP clusters mainly localized to the cap domain in dia mutant embryos. This indicated that the localization of Cpa-GFP clusters was affected in Kinesin-1 RNAi embryos.

To better describe the distribution of Cpa-GFP clusters in dia mutant embryos, I measured the number of Cpa-GFP clusters at the cap and intercap domain during mitosis and interpahse of syncytial blastoderms, and calculated densities of CpaGFP clusters at the cap $\left(\rho^{i}\right)$ and intercap $\left(\rho^{\circ}\right)$ domains. The result showed that in wild type embryos, at $0 \mathrm{~min}$, the Cpa-GFP clusters index ( $\left.\rho^{\%} \rho^{i}\right)$ was about 1 , it indicated that Cpa-GFP clusters randomly distributed on the cortex (Figure 53). At 2 min, when wild type embryos came to the interphase, the Cpa-GFP clusters index $\left(\rho \% \rho^{i}\right)$ was about 2.5, it indicated that Cpa-GFP clusters mainly localized to the intercap domain. However, in dia mutant embryos, although Cpa-GFP clusters randomly distributed on the cortex during mitosis, Cpa-GFP clusters did not migrate to the intercap domain during the interphase. Cpa-GFP clusters mainly localized to the cap domain in dia mutant embryos during the interphase. The quantification indicated that the distribution of Cpa clusters was affected in dia mutant embryos.

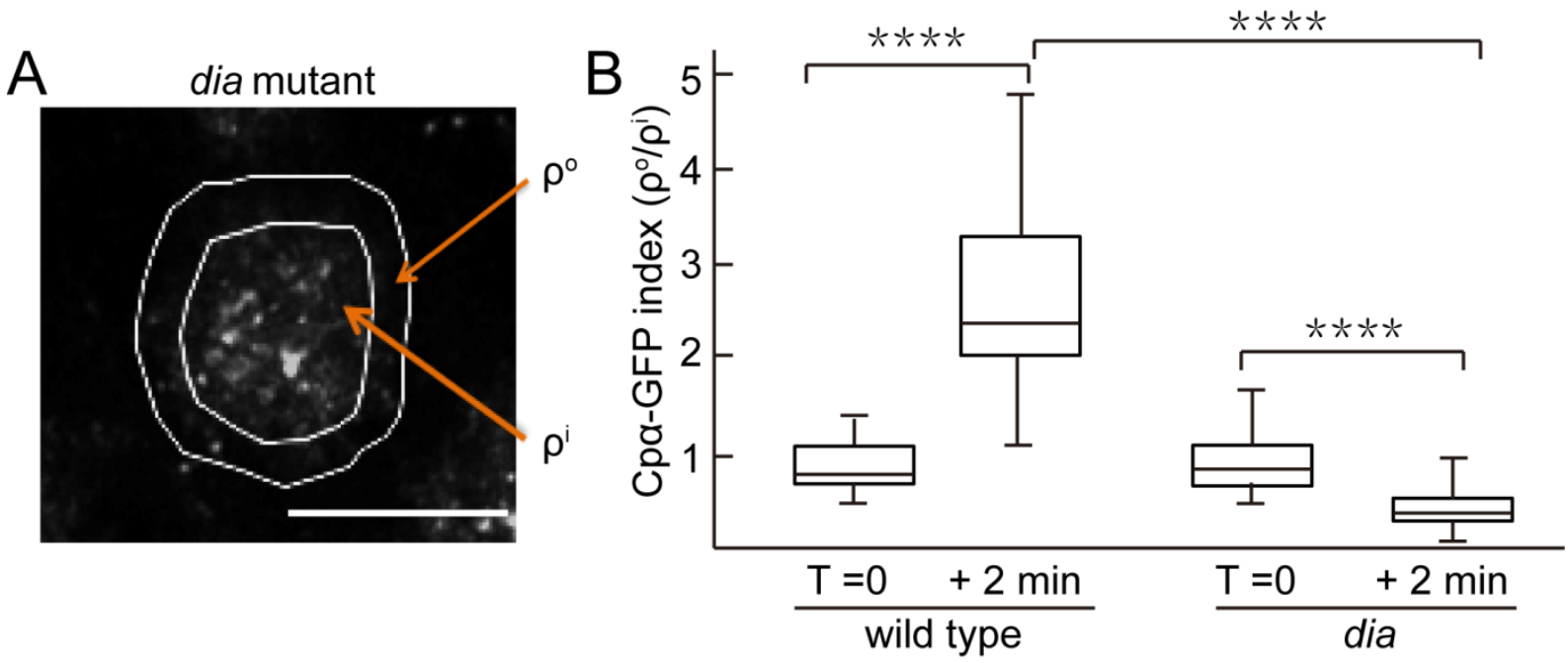

Figure 53 The Cpa distribution is affected in dia mutant embryos during syncytial interphase.

The result shows the distribution of $\mathrm{Cpa}$ clusters in $2 \mathrm{~min}$ in wild type and dia mutant embryos. (A) Schematic of the Cpa-GFP index. $\rho^{\circ}$ and $\rho^{i}$ represent Cpa-GFP clusters densities at the intercap domain and cap domain respectively. $\rho=$ (the number of Cpa-GFP clusters)/(the area). (B) Quantification of Cpa-GFP index in wild type and dia mutant embryos. The distribution of Cpa clusters is reorganized during the syncytial interphase, they mainly accumulate at the intercap domain. While Cpa clusters localize to the cap domain in dia mutant. Error bars indicate the minimum and maximum values, edges of boxes represent 25 th and 75 th percentile values respectively. ${ }^{* \star *}, p<0.0001$. The $P$ value is calculated from the paired Student's t-test. Scale bar:10 $\mu \mathrm{m}$.

Live images of the Cpa-GFP clusters distribution during cellularization in wild type 
and dia mutant embryos were recorded as well. The time when new nuclei formed was defined as $0 \mathrm{~min}$. The result showed that, in wild type, Cpa-GFP clusters mainly localized to the intercap region, and Cpa-GFP clusters were observed at $5.0 \mu \mathrm{m}$ depth in embryos at the onset of cellularization (2 $\mathrm{min})$. During the later stage of cellularization (6 min), Cpa-GFP clusters migrated deeper into embryos (Figure 54). However, in dia mutant embryos, Cpa-GFP clusters mainly localized to the surface (0-1 $\mu \mathrm{m})$ of dia mutant embryos during cellularization.
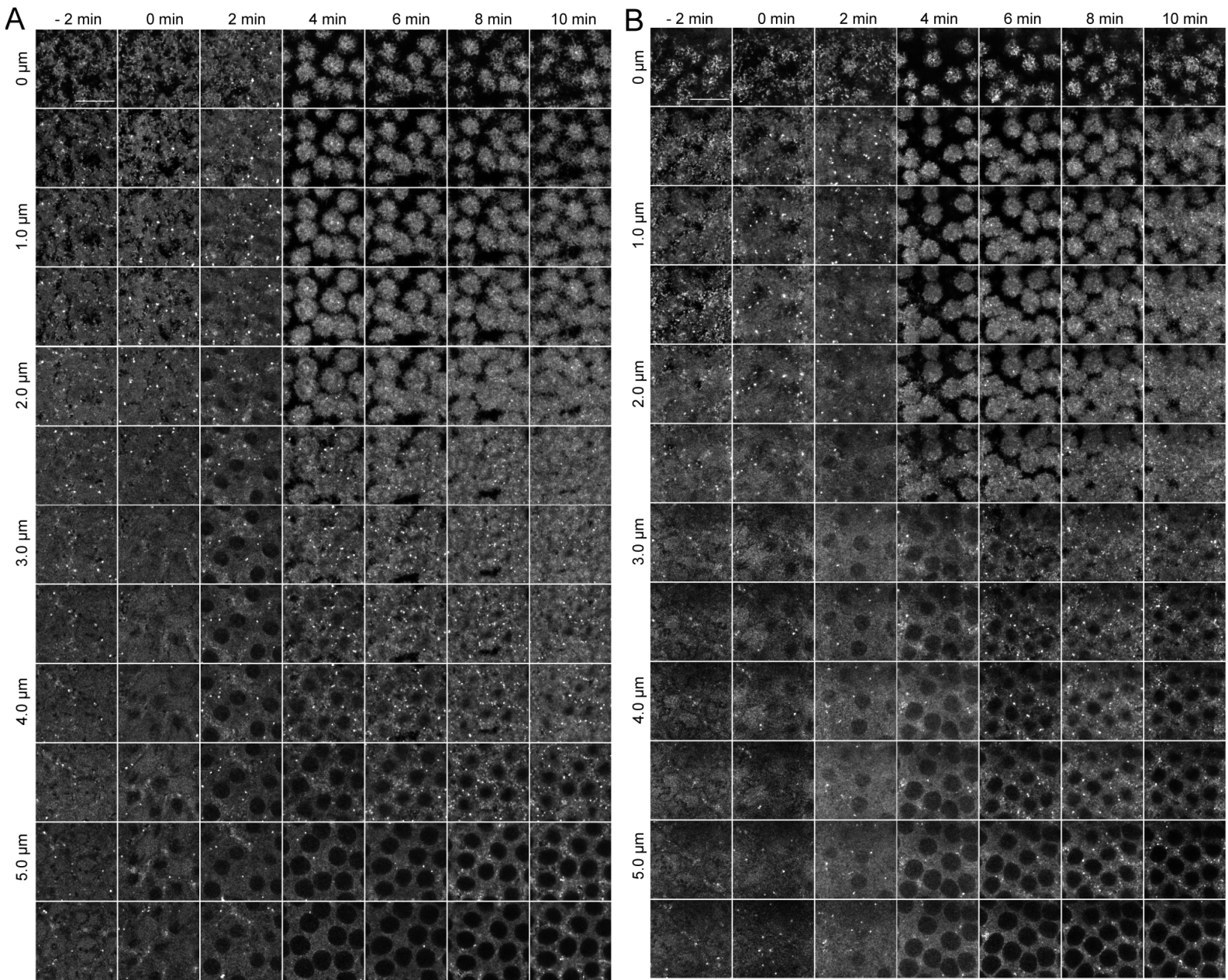

Figure 54 Dia is required for the distribution of Cpa-GFP clusters during cellularization.

The result shows the distribution of Cpa-GFP clusters in wild type and dia mutant embryos during the mitosis of cell cycle $13(-2-0 \mathrm{~min})$ and cellularization (2-10 $\mathrm{min})$. (A) The distribution of Cpa-GFP clusters in wild type embryos during cellularization. (B) The distribution of Cpa-GFP clusters in the dia mutant embryos during cellularization. In dia mutant, the distribution of Cpa-GFP clusters is affected, they mainly stay at the cap region. Z-stack size of each step is $0.5 \mu \mathrm{m}$ and the time interval is $2 \mathrm{~min}$. Scale bar: $10 \mu \mathrm{m}$.

To better understand the Cpa-GFP clusters distribution in dia mutant during cellularization, quantification of Cpa-GFP clusters densities in wild type and dia mutant embryos at the cap and furrow canal were performed (Figure 55). The quantification showed that at the onset of cellularization (0 min), in wild type embryos, 
Cpa-GFP clusters unified distributed on the embryo cortex. After 2 min, the density of Cpa-GFP clusters at the furrow canal was significantly higher than the cap region, it indicated that Cpa-GFP clusters migrated to the furrow canal during cellularization. However, In dia mutant embryos, although the Cpa-GFP clusters unify distributed at the onset of cellularization (0 min), the density of Cpa-GFP clusters at the cap domain was significantly higher than at the furrow canal after 2 min. Taken together, results about the Cpa-GFP clusters distribution in dia mutant indicated that the distribution of Cpa-GFP clusters depends on Dia.

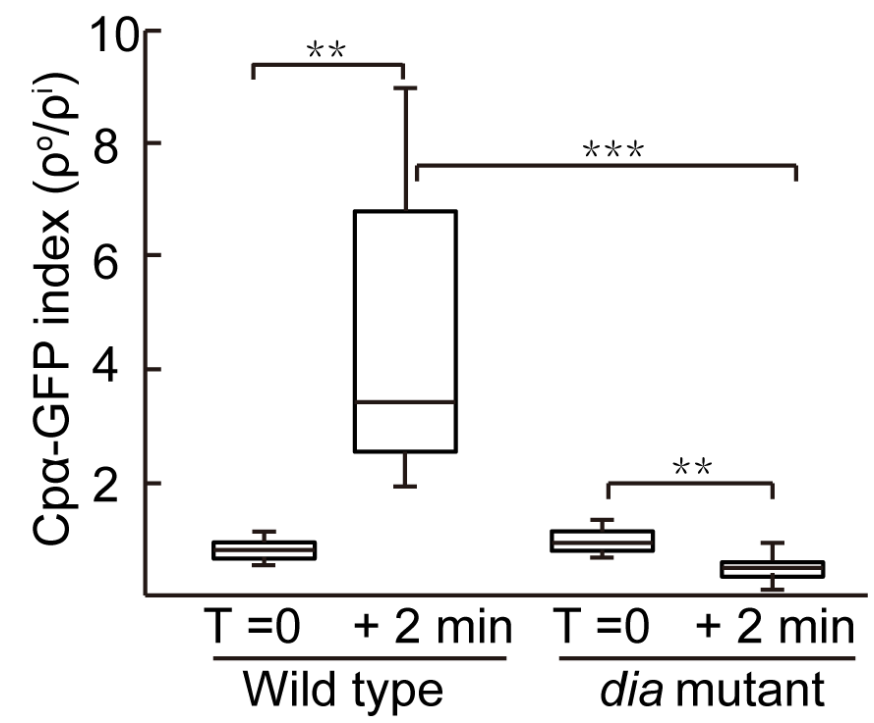

Figure 55 The Cpa-GFP clusters distribution is affected in dia mutant during cellularization.

Quantification of the Cpa-GFP clusters distribution in wild type and dia mutant embryos in 2 min during cellularization (both are 4 embryos, 4 measurements for each embryo ). $\rho=$ (the number of Cpa-GFP clusters)/(the area). Error bars indicate the minimum and maximum values, the edges of boxes represent 25 th and 75 th percentile values respectively. ${ }^{* *}$ means $p<0.01,{ }^{* * *}$ means $p<0.001$. The $\mathrm{P}$-value is calculated from the paired Student's t-test.

\subsection{APC2 coprecipitates with Kinesin-1}

Previous results that I got in this study indicated that the microtubule plus end mainly accumulates at the cap region during the interphase and Kinesin-1 can disrupt the polarity of F-actin cap. All these results made me wonder about linkages between microtubules and microfilaments. APC2 is one candidate, it belongs to tumor suppress adenomatous polyposis coli (APC) family, which has been suggested to interact with microtubules and microfilaments (Deka et al., 1998; Watanabe et al., 2004; Zumbrunn et al., 2001). APC2 regulates the organization of actin, the F-actin ring is incomplete and the furrow extension is failed in APC2 mutant embryos (Webb et al., 2009). 
To get an insight of the connection between Kinesin-1 and APC2, immunostaining of APC2 in wild type and Kinesin-1 RNAi was performed. The result showed that, in wild type embryos, APC2 accumulated to the cap domain during the interphase (Figure 56), while these separated caps could not be observed in Kinesin-1 depleted embryos. During mitosis and cellularization, APC2 invaginated with cell membrane in wild type, but APC2 stuck at the cortex in Kinesin-1 RNAi embryos. This result indicated that Kinesin-1 was required for the APC2 localization.
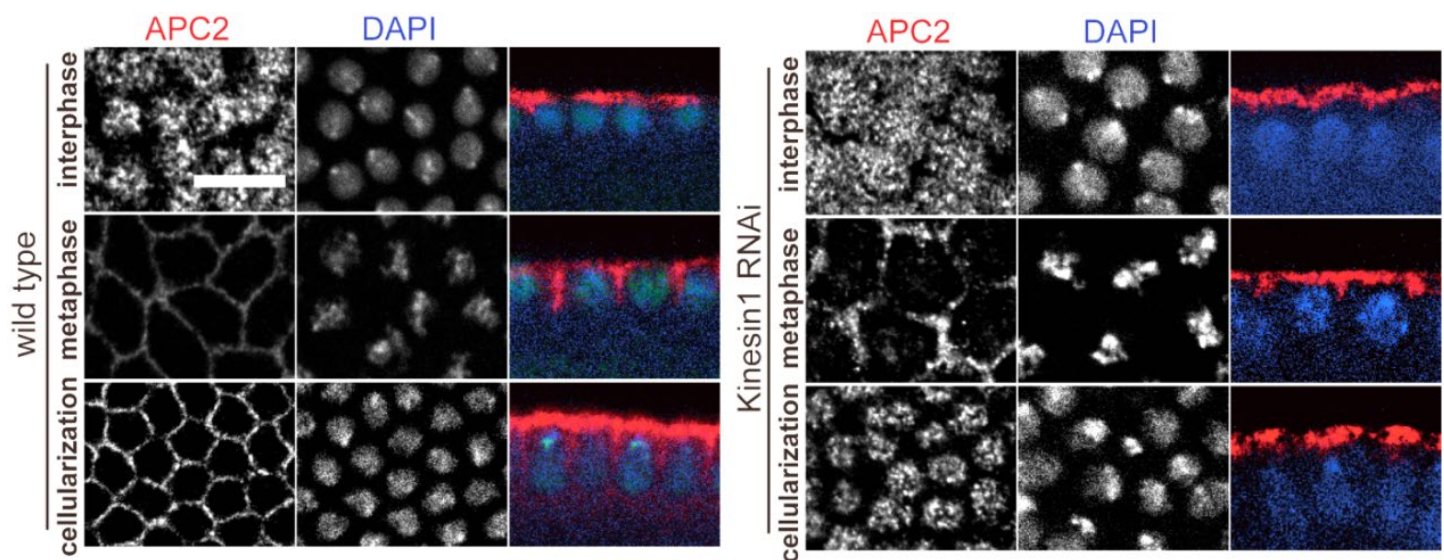

Figure 56 The APC2 localization is affected in Kinesin-1 RNAi embryos.

The result shows the APC2 (red) localization in wild type and Kinesin-1 RNAi embryos in different phases. Images show the APC2 localization during interphase, metaphase, and cellularization of wild type and Kinesin-1 RNAi embryos. Nuclei are stained with DAPI. Scale bar: $10 \mu \mathrm{m}$.

The immunostaining of APC2 in wild type embryos indicated that, APC2 localized to the cap domain during the interphase, which is similar to the localization of Kinesin-1. The previous report indicated that APC2 has a connection with microtubules (L. K. Su et al., 1995, p. 2).

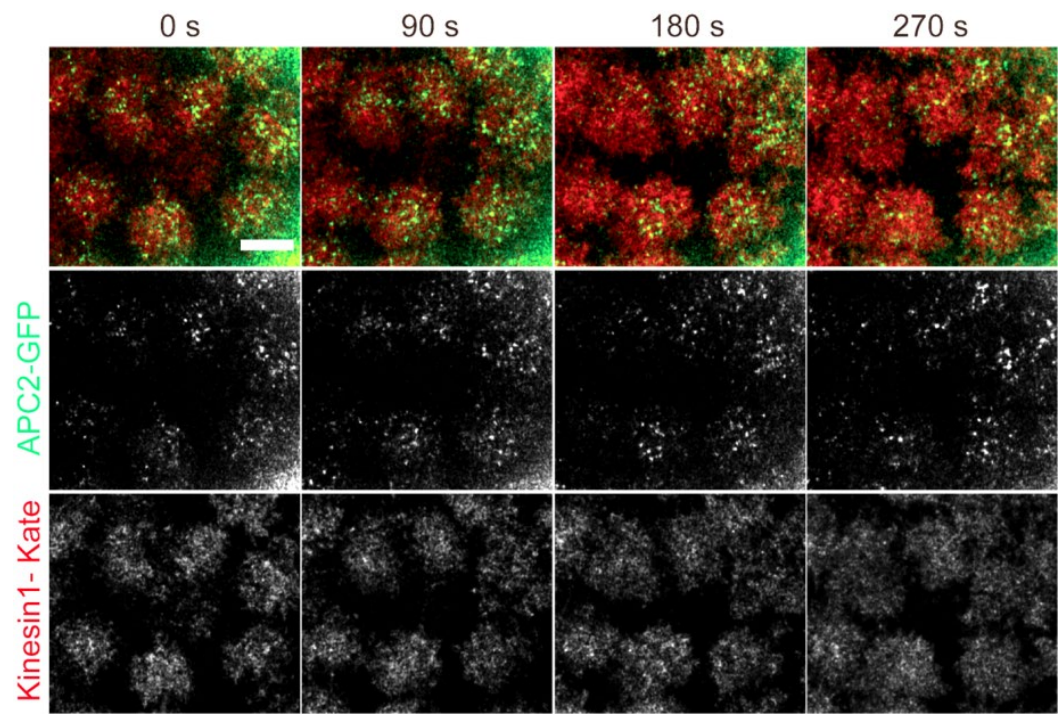

Figure 57 APC2 has an overlap with Kinesin-1.

Live images of APC2-GFP (green) and Khc-Kate (red) localization in $270 \mathrm{~s}$ during interphase. APC2GFP and Khc-Kate have an overlap at the same layer of embryos. Scale bar: $10 \mu \mathrm{m}$. 
To better understand if Kinesin-1 and APC2 have an overlap during interphase, embryos expressing Khc-Kate and APC2- GFP were utilized. The result indicated that both Khc-Kate and APC2- GFP localized to the cap domain (Figure 57), APC2GFP and Khc-Kate had overlap at the cap region during the interphase (0-270 s).

Since APC2-GFP and Khc-Kate had an very stable overlap during interphase, it is reasonable to check whether APC2 and Kinesin-1 have physical interaction. To achieve this goal, I did immunoprecipitation of APC2 and Kinesin-1. Firstly, the KhcGFP protein expression in Khc-GFP embryos was checked. The result showed that there was an abundant expression of Khc-GFP in Khc-GFP embryos (Figure 58A).

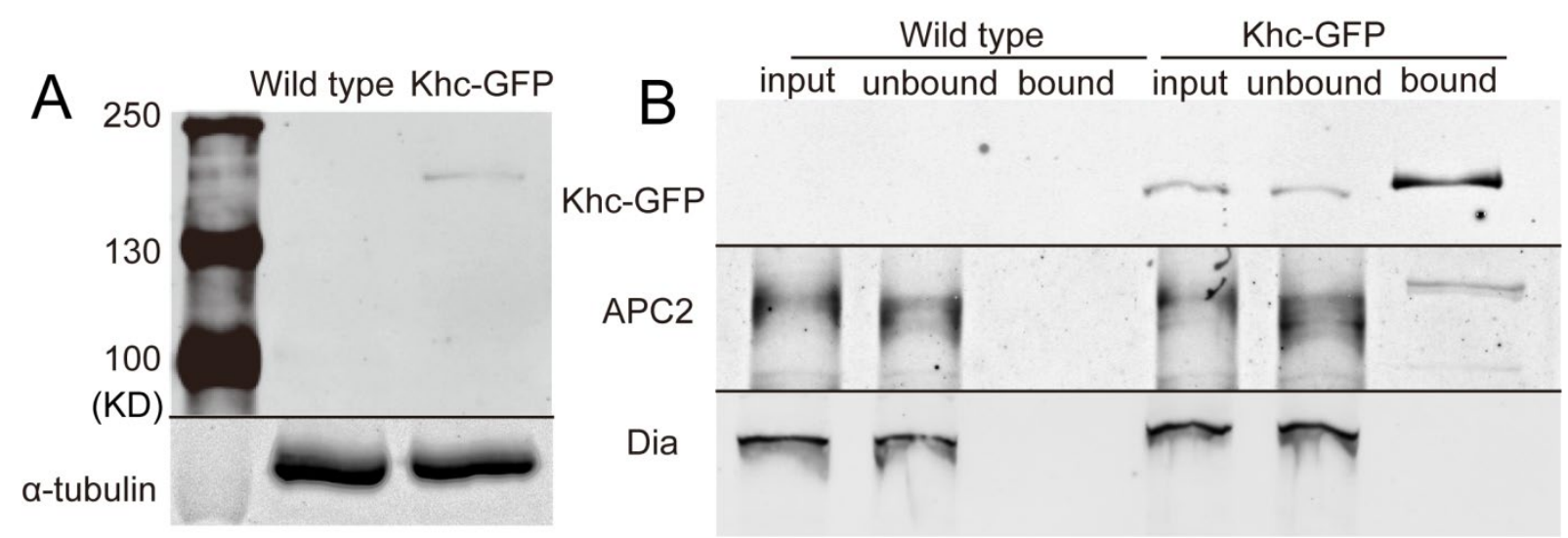

Figure 58 Kinesin-1 physically interacts with APC2.

(A) Western blot shows the Khc-GFP expression in Khc-GFP embryos. (B) Khc-GFP interacts with APC2, but not with Dia.

In immunoprecipitation experiment, GFP-trap beads were used. The result showed that, in wild type embryos, there was no Khc-GFP band in all groups and APC2 bands only appeared in input and unbound groups (Figure 58B). For Khc-GFP embryos, Khc-GFP bands could be found in all three groups, proving that GFP-trap beads work. APC2 band also could be observed in the bound group in Khc-GFP embryos, indicated Khc-GFP physically interacts with APC2.

\subsection{APC2 is required for the membrane ingression during cellularization, but it is not the linker between Kinesin-1 and cortical polarization}

As APC2 physically interacts with Kinesin-1, it is reasonable to check whether the cellularization was affected in APC2 mutant embryos. To confirm this, I got two APC2 
truncation fly lines, APC2 g10 and APC2 d40. For the APC2 g10 mutant, stop codon is inserted into APC2 at the 383th amino acid position; for the APC2 d40 mutant, stop codon is inserted into APC2 at the 677th amino acid position.

Western blot of the APC2 protein in APC2 g10 and APC2 d40 homozygous embryos was conducted (Figure 59). The result showed that only APC2 full-length protein and APC2 d40 truncation protein can be detected, as the APC2 antibody I used can detect APC2 from the 400th amino acid to the C-terminal. The APC2 protein was changed in APC2 g10 and APC2 d40 homozygous embryos. To check the function of APC2 in cortical polarization, I used the APC2 d40 homozygous for the following experiments.

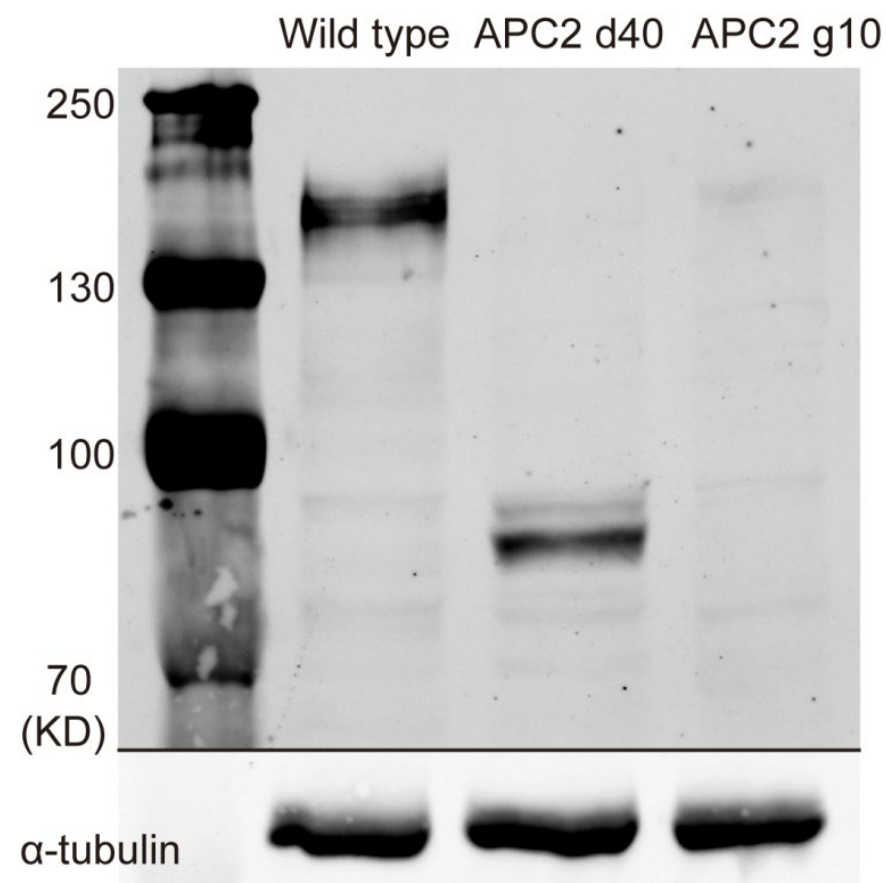

Figure 59 The APC2 protein is changed in different APC2 truncation embryos.

Western blot shows no APC2 band in APC2 g10 because the APC2 antibody used in this experiment can detect the C-terminal of APC2 (from the 400th aa to C-terminal). The a-tubulin expresion is used as control in wild type embryos and different APC2 truncations embryos.

To check whether APC2 mutation has an impact on cellularization of Drosophila embryos, DIC microscopy was performed in wild type and APC2 d40 truncation embryos (Figure 60). The result showed that the furrow invagination in wild type embryos was faster than APC2 d40 embryos during cellularization.

To get a better understanding of the function of APC2 in the membrane invagination, the furrow length was measured in wild type and APC2 d40 embryos. The time when nuclear elongation started was defined as $0 \mathrm{~min}$. The result showed that during 
cellularization, in $20 \mathrm{~min}$, the membrane invagination happened in both wild type and APC2 d40 embryos, but it was slightly slower in APC2 d40 embryos (Figure 61).

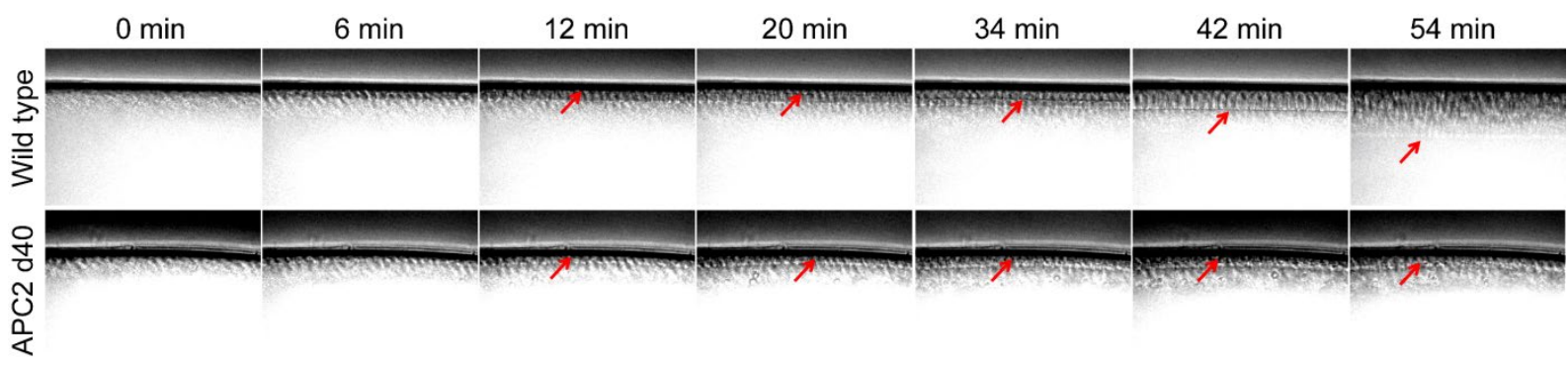

Figure 60 The membrane invagination is altered in APC2 d40 embryos.

The result shows the membrane invagination in wild type and APC2 d40 embryos at the indicated time points during cellularization. The furrow front is much deeper in wild type than in APC2 d40 embryos. Red arrows indicate fronts of the furrow. Scale bar: $20 \mu \mathrm{m}$.

At $42 \mathrm{~min}$, a significant difference of the furrow length between wild type and APC2 d40 could be observed, the furrow was much longer in wild type than in APC2 d40. The result indicated that APC2 is required for the cellularization process.

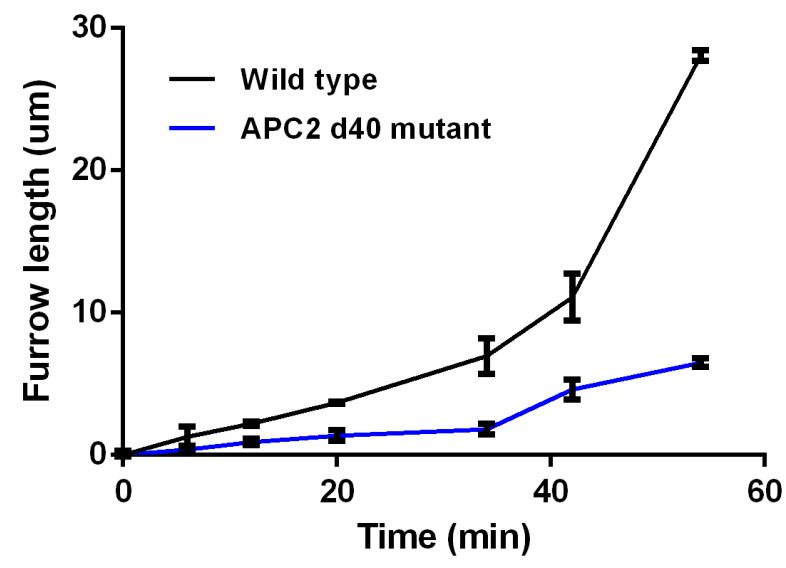

Figure 61 The furrow invagination is affected in APC2 d40 embryos during cellularization.

The result shows the furrow length at the indicated time points during cellularization. Furrow length in wild type and APC2 d40 embryos (both are 3 embryos) are measured, spots represent the median of furrow length. Error bars represent s.e.m.

To get detailed information about how cellularization is affected by APC2, immunostainings of Slam, Canoe, Bazooka, and Dlg were performed respectively. In these experiments, His-GFP and APC2 d40 embryos were stained in the same tube. The results showed Slam (Figure 62), Canoe (Figure 63), Bazooka (Figure 64) and Dlg (Figure 65) localized to basal, subapical and lateral domain respectively in both wild type and APC2 d40 embryos. Immunostaining results indicated although the APC2 mutation induced cellularization defects, the localization of cortical components was not affected. This is different from the cellularization defects in Kinesin-1 RNAi 
embryos.

3.10.1 The cortical polarization is not relied on APC2
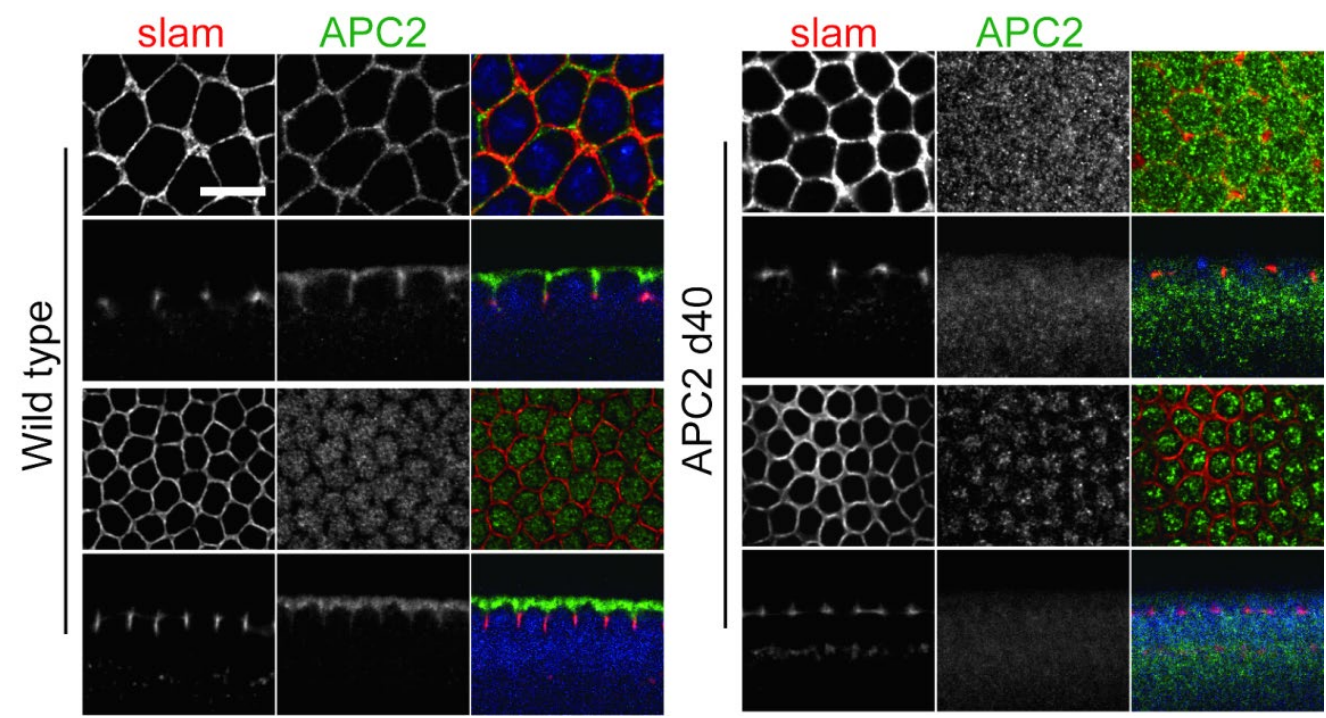

Figure 62 APC2 is not required for the Slam localization.

The result shows Slam (red) and APC2 (green) localization in wild type and APC2 d40 embryos during syncytial and cellularization stages. Nuclei are stained with DAPI. Scale bar: $10 \mu \mathrm{m}$.

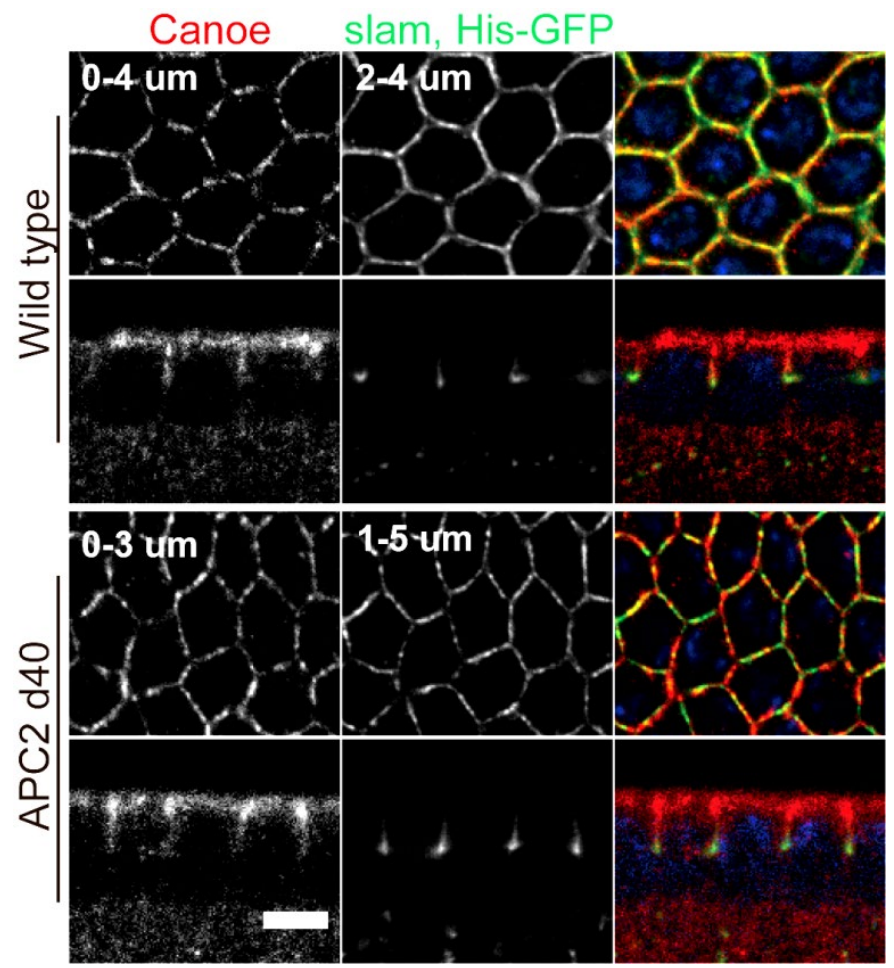

Figure 63 The Canoe localization is not affected in APC2 d40 embryos.

The localization of Canoe (red) and Slam (green) in both wild type and APC2 d40 embryos during cellularization. The result indicates that APC2 is not required for the Canoe localization. Wild type and APC2 d40 embryos are stained in the same tube. Ranges indicate projection depths of Canoe and Slam in wild type and APC2 d40 embryos. Scale bar: $5 \mu \mathrm{m}$. 


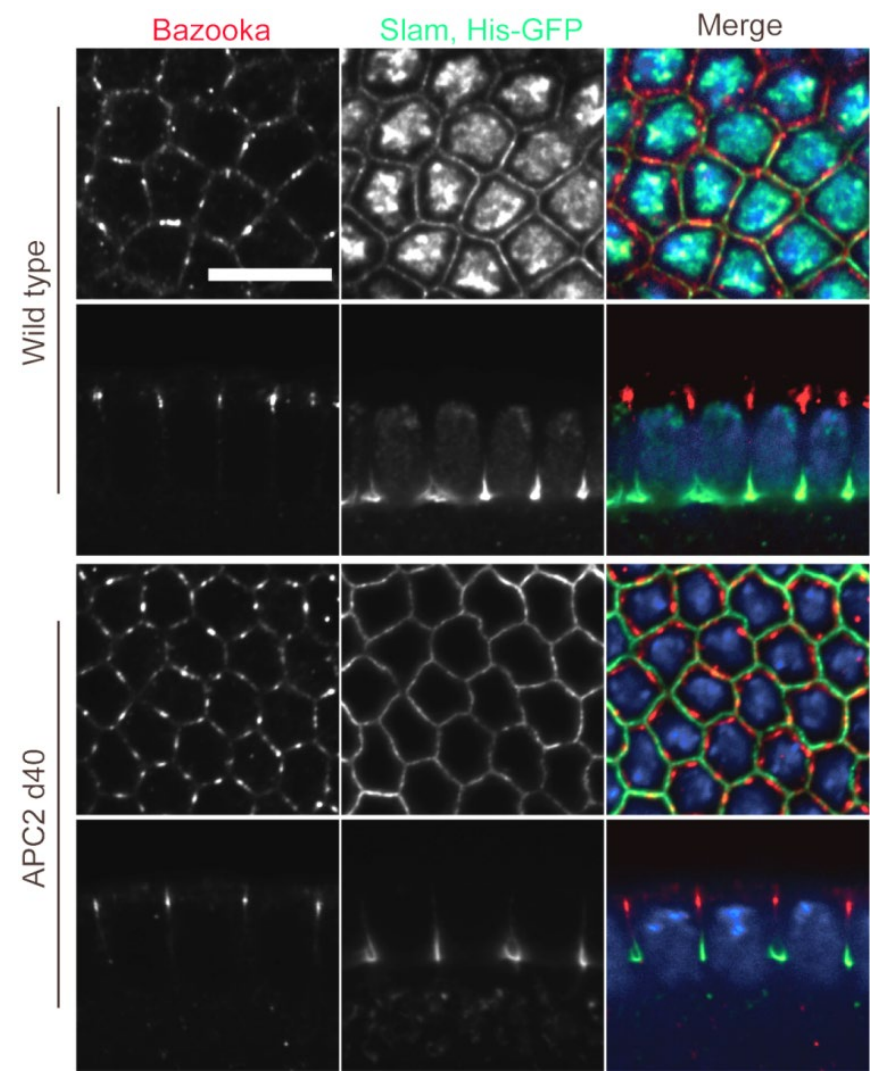

Figure 64 The localization of Bazooka is not affected in APC2 d40 embryos.

The result shows Bazooka (red) and Slam (green) localization in wild type and APC2 d40 embryos during cellularization. Bazooka localizes to the subapical domain in wild type embryos during cellularization. Bazooka localizes to the downstream of Canoe, wild type and APC2 d40 embryos are stained in the same tube. Scale bar: $10 \mu \mathrm{m}$.

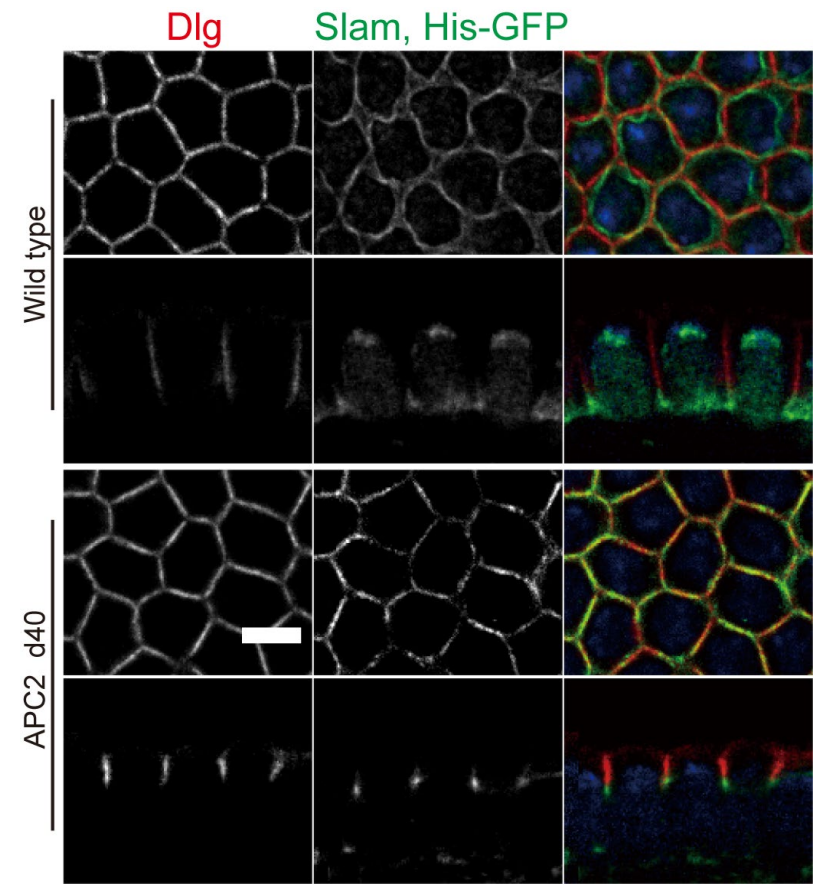

Figure 65 The localization of Dlg is not altered in APC2 d40 embryos.

The result shows the localization of Dlg (red) and Slam (green) in both wild type and APC2 d40 embryos during cellularization. The result indicates that APC2 is not required for the Dlg localization. Wild type and APC2 d40 embryos are stained in the same tube. Scale bar: $5 \mu \mathrm{m}$. 
3.10.2 Accumulations of Slam and Amphiphysin at the furrow tip are disrupted in APC2 d40 embryos

Immunostainings of membrane components indicated that different domains in APC2 d40 embryos during cellularization were not affected. To figure out how the cellularization was affected in APC2 d40 embryos, the amount of Slam and Amphiphysin at furrow tips during cellularization were checked. Amphiphysin localizes to the basal domain, and it can recruit and stabilize cortical proteins (Zelhof et al., 2001). I stained His-GFP and APC2 d40 embryos together in the same tube. The result showed Slam and Amphiphysin localized to the basal domain in both wild type and APC2 d40 embryos, but Slam and Amphiphysin fluorescent intensities at the furrow tip were stronger in wild type than in APC2 d40 embryos (Figure 66A). To better understand whether Slam and Amphiphysin at the basal domain were affected in APC2 d40 embryos, fluorescent intensities of Slam and Amphiphysin at the basal domain were measured. The quantification showed that fluorescent intensities of Slam and Amphiphysin at the basal domain were significant higher in wild type than APC2 d40 embryos (Figure 66B). The result indicated that APC2 is required for Slam and Amphiphysin accumulation at the furrow tip.
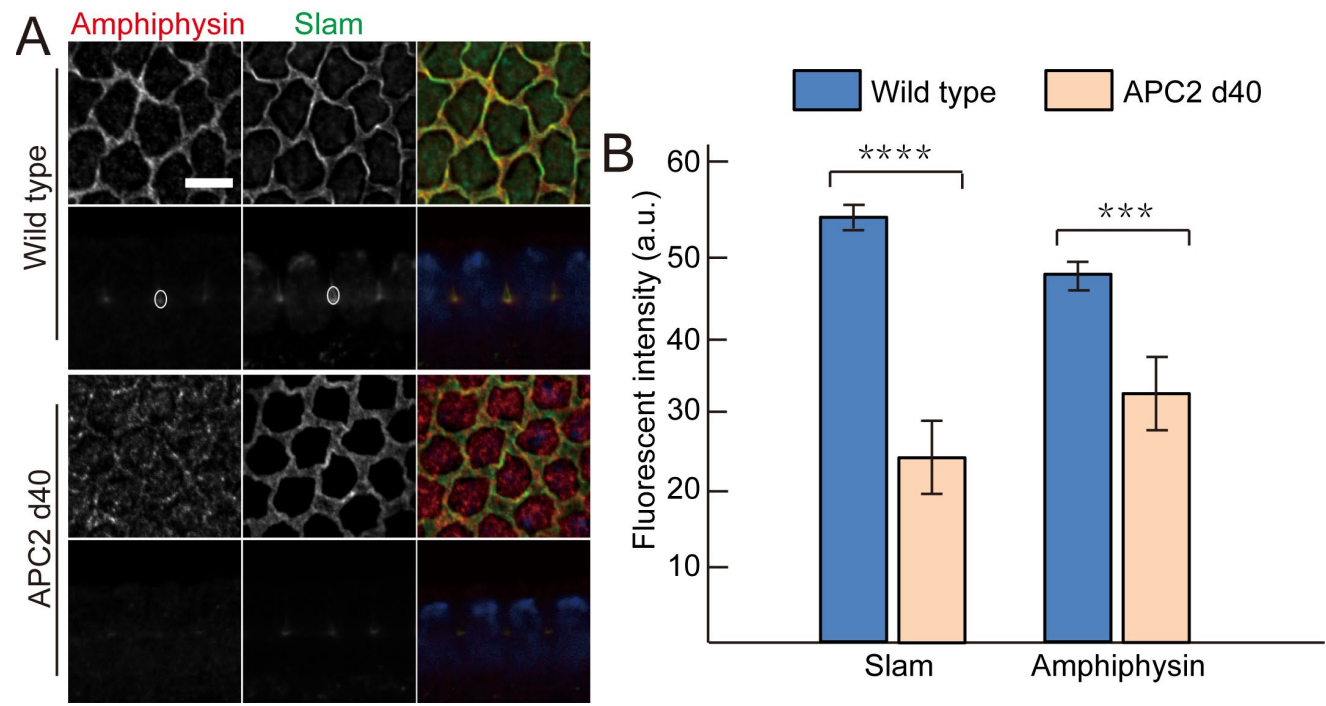

Figure 66 Accumulations of Slam and Amphiphysin at the furrow tip are decreased in APC2 d40 embryos.

(A) Accumulations of Amphiphysin (red) and Slam (green) in wild type and APC2 d40 embryos during cellularization. Amphiphysin localizes to the basal domain during cellularization. Bright circles are the quantified domains. (B) Quantifications of Amphiphysin and Slam fluorescent intensities at furrow tips. Error bars represent s.e.m. ${ }^{* * *}$ means $p<0.001,{ }^{* * * *}$ means $p<0.0001$. The P-value is calculated from the paired Student's t-test. Scale bar: $5 \mu \mathrm{m}$.

Taken together, the results obtained from APC2 d40 embryos indicated that APC2 
promoted accumulations of Slam and Amphiphysin at the basal domain during cellularization, the amounts of Slam and Amphiphysin at the basal domain was important for cellularization. 


\section{Discussion}

Kinesin- 1 is a (+)-end motor protein, it tracks along microtubule from its minus end to the plus end, which is required for multiple cellular processes, such as sliding of microtubules and cytoplasmic streaming in Drosophila oocyte (Lu et al., 2016; Palacios and St Johnston, 2002), the posterior localization of oskar mRNA in Drosophila oocyte (Brendza et al., 2000; Gáspár et al., 2017). Kinesin-1 is also required for the membrane ingression and the nuclear elongation in Drosophila embryos during cellularization (Winkler et al., 2015). These reports indicate that different localization of Kinesin-1 has different functions (Du and Su, 2019). To better understand the mechanism about how Kinesin-1 affects the cellularization process in Drosophila embryos, it is vital to get the knowledge of the localization of Kinesin-1 in syncytial and cellularization stages. Here I could show that, in Drosophila syncytial blastoderms, Kinesin-1 is found at the cap domain during the syncytial interphase, where F-actin, Moesin, ELMO/Sponge accumulate. During mitosis, Kinesin-1 localizes to the metaphase furrow. As the metaphase furrow elongates and contracts in a few minutes during mitosis, Kinesin-1 is required for membrane components transporting in these two processes. During cellularization, Kinesin-1 migrates to subapical, lateral and basal domains and the amount of Kinesin-1 at the cap region decreases.

Kinesin-1 localizes to the cap domain during the syncytial interphase, the movement of Kinesin-1 depends on microtubules, this reminded me whether the cap domain accumulation of Kinesin-1 is due to the accumulation of plus ends of microtubules at the cap domain. Microtubules are polar structures and they have plus end and minus end. In terms of the nucleation center, microtubules can divide into centrosomal microtubules and non-centrosomal microtubules. In epithelial cells, for centrosomal microtubules, the nucleation center is centrosome, it localizes to the minus end of microtubules, the plus end of microtubules points to the cell membrane, where the actin network exists. Former publications indicated that both plus end and minus end of microtubules can connect with F-actin filaments via different proteins (Goodwin and Vale, 2010; Nashchekin et al., 2016; Webb et al., 2009). For example, EB1, CLIP-170, and APC proteins bind to the plus end of microtubules and connect to actin filaments (Dogterom and Koenderink, 2019a). 
For non-centrosomal microtubules, previous studies indicated that Patronin localizes to the minus end of microtubules and acts as the nucleation center to promote the polymerization of non-centrosomal microtubules (Goodwin and Vale, 2010; Khanal et al., 2016; Nashchekin et al., 2016). Patronin and Shot form protein complex to start the microtubule nucleation, the connection between Shot and actin network helps microtubules anchoring on the cell membrane (Dogterom and Koenderink, 2019a). There is no y-tubulin exists in the nucleation center (Nashchekin et al., 2016). In my study, I could find that in Drosophila embryos, EB1 mainly localized to the cap domain during the interphase, had an overlap with Moesin. The result indicated that plus ends of microtubules mainly accumulated beneath the cap domain. Based on previous reports and my results, I suppose interactions between microtubules and Factin filaments may help Kinesin-1 localizes to the cap domain during the syncytial and cellularization stages (Figure 63).

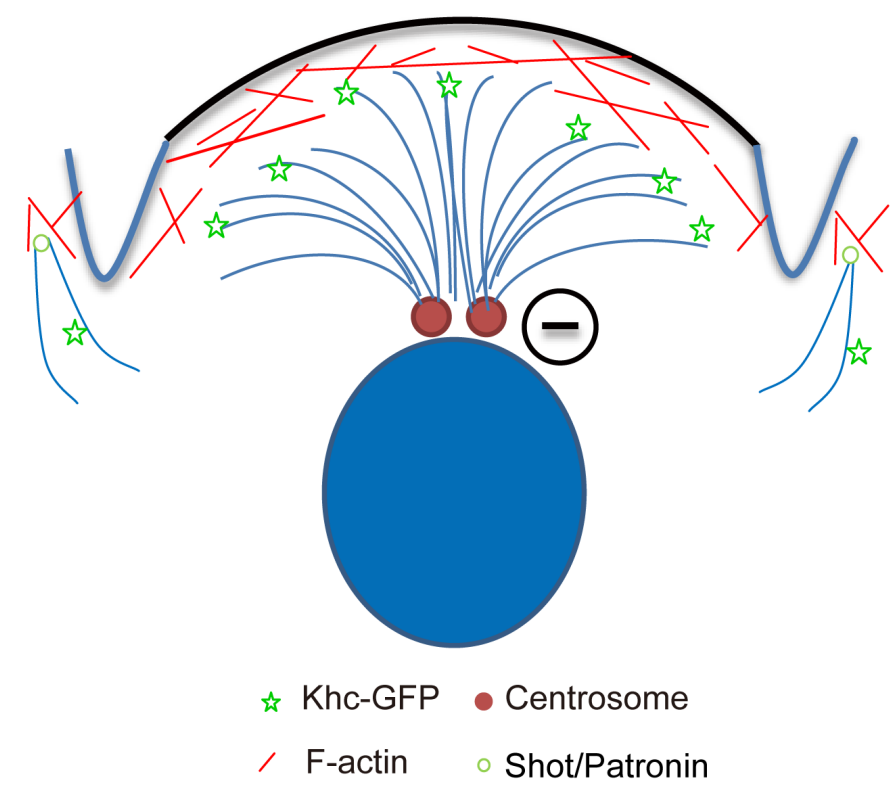

Figure 67 Schematic of the Khc-GFP localization in Drosophila syncytial blastoderms.

During the interphase of syncytial blastoderms, Kinesin-1 and F-actin mainly localizes to the cap domain, Slam localizes to the intercap domain. Interactions between microtubules and F-actin filaments promote cortical localization of Kinesin-1. For centrosomal microtubules, different proteins such as EB1, CLIP-170, and APC proteins bind to the plus end of microtubules, mediate microtubules interacting and anchoring on the F-actin network. For non-centrosomal microtubules, the minus end of non-centrosomal microtubules interacts with F-actin through Patronin/Shot complex. The anchoring of microtubules to the F-actin network helps Kinesin-1 localizes to the cortex of embryos.

With Spatial-temporal regulation, the cortex of Drosophila early embryos divides into different domains, which are defined by distinct localization of proteins (Schmidt and Grosshans, 2018). During the interphase of syncytial blastoderms, the cortex of 
embryos divides into cap domain and intercap domain. F-actin, Canoe, and ELMO/Sponge complex localize to the cap domain, Slam and Myosin II localize to the intercap domain (Lecuit et al., 2002; Manning et al., 2019; Schmidt et al., 2018). During the cellularization stage, the cortex of embryos separates into apical, subapical, lateral, and basal domains. Canoe and ELMO/Sponge complex localize to the subapical domain and the basal domain is labeled with Slam, Myosin II and Factin (Schmidt and Grosshans, 2018; Sen et al., 2012; Wei et al., 2015). The asymmetric distribution of proteins in different domains is vital for cell differentiation and Drosophila early embryonic development (Munro et al., 2004; Schmidt and Grosshans, 2018).

For example, the localization of ELMO at the cap domain is important for the F-actin organization, the formation of F-actin cap is affected in ELMO mutant embryos, the cortical polarization and cellularization are affected as well (Schmidt et al., 2018). Furthermore, the basal localization of Slam is essential for cellularization. In slam mutant embryos, the amount or the function of Slam protein is affected, this induces the disruption of membrane invagination during cellularization and embryos cannot go through cellularization (Acharya et al., 2014; Lecuit et al., 2002; Yan et al., 2017). The asymmetric distribution of proteins and the cortical differentiation in Drosophila embryos during cellularization are regulated by different protein candidates (Figure 64).

The defects of membrane ingression was observed in Kinesin-1 RNAi and the defects of cellularization in Kinesin-1 RNAi embryos is similar to slam mutant embryos. In slam mutant embryos, the localization of Slam protein at the basal domain is affected during cellularization (Lecuit et al., 2002; Yan et al., 2017). I assumed that the defects of membrane ingression during cellularization in Kinesin-1 RNAi is also induced by the disruption of Slam at the basal domain. Apart from the basal localization of Slam protein, the basal localization of slam mRNA is crucial for cellularization as well, as the rapid accumulation of Slam protein at the basal domain depends on the local translation of slam mRNA at the basal domain during cellularization (Acharya et al., 2014; Yan et al., 2017). Meanwhile, the basal localization of Slam protein is required for the localization of slam mRNA at the basal domain. Results about Slam from live images and immunostaining showed that the localization of Slam is affected during cellularization. 


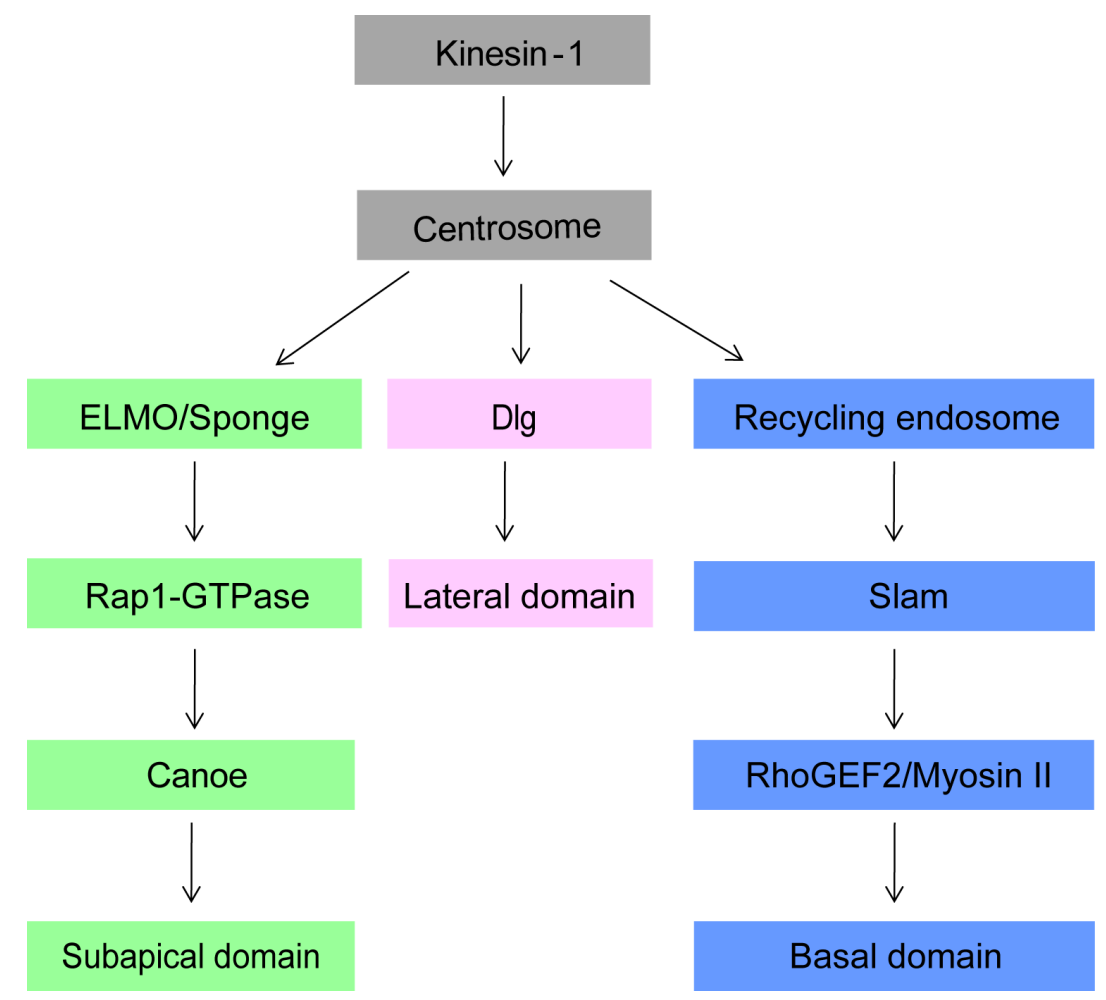

Figure 68 The formation of cortical domains during Drosophila early embryonic development is regulated by Kinesin-1.

Schematic of signaling pathways of subapical (green), lateral (pink), and basal (blue) domains formation during cellularization stage. (1) Kinesin-1 localizes to the upstream of centrosomes, it regulates the fluctuation of centrosomes (Winkler et al., 2015). (2) ELMO/Sponge complex regulates the localization of Canoe and influences the subapical domain formation. In Kinesin-1 RNAi embryos, the localization of ELMO/Sponge complex is affected. (3) Centrosomes affect the lateral domain formation, the localization of Dlg at the lateral domain is affected when centrosome is disrupted (Wilson, 2011). (4) The localization of Slam at the basal domain is regulated by the recycling endosome, the basal localization of Slam regulates the localization of RhoGEF2 and Myosin II in Drosophila embryos (Großhans et al., 2005; Lecuit et al., 2002). Kinesin-1 localizes to the upstream of the recycling endosome, as Nuf is slightly affected in Kinesin-1 RNAi embryos.

But in syncytial blastoderms, the localization of Slam protein is not affected. This indicates that the mislocalization of Slam is not the main cause for the failure of cellularization in Kinesin-1 RNAi embryos.

During cellularization, the time point that different cortical domains come out is slightly different, the subapical domain comes out slightly later than the basal domain (Schmidt et al., 2018). As the basal domain is affected in Kinesin-1 RNAi embryos, I wondered whether the subapical domain was affected as well in Kinesin-1 RNAi embryos. Live images of Canoe and ELMO/Sponge could show that the newly formed subapical domain is affected in Kinesin-1 depleted embryos. Canoe and ELMO/Sponge complex are mislocalized in Kinesin-1 RNAi embryos. Live images indicated that the localization of Canoe and ELMO/Sponge complex is affected 
during the syncytial stage, the localization of ELMO/Sponge at the rim of the cap in Kinesin-1 RANi embryos was not as restricted as wild type. Live images also showed that the Canoe localization at the subapical domain during cellularization is affected in Kinesin-1 RNAi embryos, Canoe migrates from the cap domain to the subapical domain during cellularization (Schmidt et al., 2018). Mislocalization of ELMO/Sponge complex and Canoe during the syncytial stage may induce the defection of Canoe and ELMO/Sponge complex at the subapical domain during cellularization.

The localization of cortical proteins not only depends on the protein-protein regulation but also relies on the protein transporting. For example, the defects of the recycling system influences the localization of cortical components. Endosomal membrane recycling system sorting and transporting proteins such as ion channels, surface receptors, and membrane proteins back to the membrane. The recycling endosome is required for the localization of Slam protein at the basal domain during cellularization (Acharya et al., 2014). Nuf protein, as a marker protein of recycling endosome (Calero-Cuenca et al., 2016; Hickson et al., 2003; Riggs et al., 2003), was used to check recycling endosomes in Kinesin-1 RNAi embryos. The result proved that the recycling endosome is slightly different in Kinesin-1 RNAi embryos. Tubular transport in endosomal membrane recycling system depends on motor proteins such as KIF13 and Myosin 5B (Delevoye et al., 2014; Goldenring, 2015; Müller et al., 2008), while Kinesin-1 depletion affects the F-actin polarization in embryos, this might partially explain Kinesin-1 depletion influences membrane components transporting from endosomes to membrane in Drosophila embryos. However, this is not the main cause for the disruption of cortical polarization in the Kinesin-1 RNAi embryos, since membrane components localize to the cortex of syncytial blastoderms.

Apart from the endosomal membrane recycling system, the motor protein dependent cargo transporting is also important for the cortical components localization. Kinesin1 is one of the candidates, Kinesin-1 transports cargos that contain cortical proteins along microtubules to the cell membrane (Hirokawa et al., 2009). Since the cortex of Drosophila embryos is highly dynamic during early embryonic development (Mavrakis et al., 2009), I proposed the defection of protein transport is the main reason that hampers cortical polarization in Kinesin-1 RNAi embryos. This was checked by the FRAP experiment. The previous study indicated that GFP-Slam fluorescent intensity recovers completely after photobleaching at the onset of cellularization, the fluorescent intensity cannot fully recover at the bleached region during the middle of 
cellularization (Acharya et al., 2014), so I checked dynamics of GFP-Slam at the onset of cellularization in wild type and Kinesin-1 RNAi embryos. There is no significant difference in the recovery speed of GFP-slam fluorescent intensity in Kinesin-1 RNAi compared to wild type. The result proves that the membrane components transport by Kinesin-1 is not the main cause for the defects of cortical polarization in Kinesin-1 RNAi embryos.

Microtubules provide tracks for the Kinesin-1 movement, and microtubules are dynamic constructs, the nucleation and polymerization of microtubules are important for the function of the microtubule system (Baas et al., 2016; Basnet et al., 2018; Volkov et al., 2018). Centrosomes can initiate the cortical polarization in different ways, the microtubule-dependent signal is one of the most important ways among them (Cowan and Hyman, 2004). The centrosome is the nucleator center and the minus end for centrosomal microtubules, EB1 binds to the plus end of microtubules. The results in this study could show that centrosomes in Kinesin-1 RNAi embryos are comparable to wild type, the polymerization dynamics of microtubules in Kinesin-1 RNAi embryos is normal.

In Kinesin-1 RNAi embryos, although the cortical domains cannot be clearly separated during cellularization, components of the basal domain (Slam), subapical domain (Canoe, ELMO/Sponge complex) are stuck at the surface of embryos. One explanation about the Kinesin-1 depletion did not affect cortical components transport to the membrane is that Kinesin superfamily (KIF) proteins have redundant functions, the function of Kinesin-1 might be replaced by other KIF proteins. For example, $\mathrm{KIF} 1 \mathrm{Ba}$ and KIF5 both transport mitochondria from the minus to the plus end of microtubules (Cai et al., 2005; Nangaku et al., 1994). Another explanation about the mislocalization of cortical components is because of the failure of furrow invagination during cellularization in Kinesin-1 RNAi embryos. In this case, there is no membrane structure for the localization of cortical proteins during cellularization in the Kinesin-1 RNAi embryos.

The defects of furrow canal formation in Drosophila embryos can affect cellularization has been proved in my study. To find candidates between microtubules and F-actin filaments, I selected several candidates include APC2 protein, APC2 mutant embryos cannot go through cellularization. APC2 can connect the plus end of microtubules to the cell membrane, as $\beta$-catenin binding domain exists in APC2 (Juanes et al., 2019; Munemitsu et al., 1994; Webb et al., 2009). I found that the disruption of 
cellularization in APC2 mutant is not due to the mislocalization of cortical components, the localization of proteins in APC2 mutant are comparable to wild type.

The quantifications of Slam and Amphiphysin at the basal domain in the APC2 mutant indicated that Slam and Amphiphysin at the basal domain decrease. Amphiphysin belongs to BAR (Bin-Amphiphysin-Rvs) domain protein, BAR domain proteins are a set of proteins that have membrane deform properties (Ramjaun et al., 1997; Su et al., 2013; Takei et al., 1999; Zelhof et al., 2001). Amphiphysin is the first protein that has been identified have a function in membrane tubulation. Amphiphysin localizes to sites of endocytosis, Amphiphysin binds to Dynamin via its SH3 domain. Dynamin is also a membrane deforming protein and it has been identified as an actin regulator. Amphiphysin and Dynamin regulate membrane invagination in living cells by interacting with the F-actin cytoskeleton (Itoh et al., 2005). When cells are treated with Latrunculin B or Cytochalasin D, the actin network in cells is affected and the membrane tubulation starts to grow. Latrunculin B inhibits the actin filaments polymerization (Spector et al., 1983), Cytochalasin D binds to the plus end of actin filaments and blocks their elongation (Cooper, 1987).

Another protein that affects membrane invagination is Myosin II. Distribution of Myosin II at the cortex of Drosophila embryos is crucial for ventral furrow formation (Krueger et al., 2018). Before ventral furrow formation starts, Myosin II mainly localizes to the basal domain in Drosophila embryos. When ventral furrow formation starts, Myosin II migrates to the apical domain, the amount of Myosin II at the basal domain decreases. The translocation of Myosin II from basal domain to the apical domain is vital for ventral furrow invagination, disruption of the apical localization of Myosin II induces defects of ventral furrow formation as well as the shape of epithelial cells in embryos.

Myosin II and Amphiphysin affect the membrane invagination via their connection with the actin network, it reminded me whether Kinesin-1 also affects the F-actin network and induces defects of cellularization.

Previous publications indicated that not only microtubule-dependent Kinesin-1 but also the connection between microtubules and F-actin filaments are essential for the cell membrane formation (Dogterom and Koenderink, 2019b; Juanes et al., 2019; Nangaku et al., 1994). Drosophila embryo is a good model to figure out how microtubules affect the cortical polarization and the connection between microtubules and F-actin filaments, as microtubules and F-actin filaments exist in Drosophila early 
embryos (Harris et al., 2009). Furthermore, the cortex of Drosophila embryos is highly dynamic during the early embryonic development, the cortex of embryos divides into four domains during cellularization (Bonello et al., 2019; He et al., 2016; Kao and Megraw, 2009; Schmidt and Grosshans, 2018).

Microtubules and F-actin filaments are required for the cortical polarization. Centrosomes and its associated microtubules can deliver signals to induce cortical polarization, cytoplasmic microtubules also can improve the symmetric cleavage by moving centrosome close to the cortex (Bienkowska and Cowan, 2012; Raff and Glover, 1989b). F-actin is essential for the localization of cortical components in Drosophila embryos during cellularization, it can affect the basal localization of Peanut and Patj during Drosophila cellularization (Dominguez and Holmes, 2011; Mavrakis et al., 2009). But the connection between microtubules and F-actin filaments and how microtubules and F-actin filaments together affect cortical polarization during early embryonic development are not clear yet. As Kinesin-1 is a microtubule-dependent protein and it localizes to the cap domain during the syncytial interphase, I supposed that Kinesin-1 is a linker between microtubules and F-actin filaments.

In Drosophila early embryos, the F-actin network under the cell membrane is dynamic. In syncytial blastoderms, the organization of F-actin network is different between mitosis and interphase. F-actin filaments mainly accumulate to the cap domain and form cap structure during the syncytial interphase. During mitosis, Factin caps disassembled, F-actin filaments migrate with cell membrane to the metaphase furrow. In my study, I focused on the organization of the F-actin network during the syncytial interphase and I investigated how Kinesin-1 affects the organization of the F-actin network.

Moesin is the only ERM (Ezrin, Radixin, and Moesin) protein in Drosophila, which is essential for apical domains maintenance and cortical stability (Karagiosis and Ready, 2004), it defines the F-actin cap region during interphase. I found that EB1-GFP mainly accumulates at the cap domain during the interphase of syncytial blastoderms. This indicates that the plus end of microtubules mainly localizes beneath the cap region. The previous live images of Khc-GFP showed that Kinesin-1 accumulates at the cap domain during the interphase. Based on these results, I assumed that Kinesin-1 is required for the formation of cap domain during the interphase of syncytial blastoderms. Although cap and intercap domains are well separated in 
Kinesin-1 RNAi embryos, I could show that the localization of Utro-GFP in Kinesin-1 RNAi is different from the wild type. In Kinesin-1 RNAi embryos, the difference of actin cap shape may due to the disruption of F-actin cap contraction.

Previous publications concluded different ways of interaction between microtubules and F-actin filaments (Coles and Bradke, 2015; Dogterom and Koenderink, 2019a). Furthermore, F-actin is essential for the localization of membrane components. These reminded me that the Kinesin-1 depletion may affect microtubule-actin interaction and F-actin polarity, induce the mislocalization of cortical proteins. To test this assumption, I checked the localization of Capping proteins. Capping proteins including Capping $\alpha(\mathrm{Cp} \alpha)$ and Capping $\beta(\mathrm{Cp} \beta)$, they localize to the plus end (barbed end) of F-actin filaments and inhibit the elongation of F-actin filaments. The role of Capping proteins in the growth of the Drosophila wing has been reported (Amândio et al., 2014). Results in this study could prove that the F-actin cap is a polar structure and the organization of F-actin caps was affected in Kinesin-1 RNAi embryos.

In muscle cells, Myosin and actin filaments form a well-organized structure and generate force for muscle contractility. During the force generation in muscle cells, Myosin binds to and migrates along antiparallel F-actin filaments. Previous reports have shown, in Drosophila embryos, Myosin helps epithelial apical constriction in the ventral formation during gastrulation (Coravos and Martin, 2016; Lv and Großhans, 2016). My hypothesis was Kinesin-1 has a function in F-actin cortex polarization, Factin cap constriction is affected in Kinesin-1 depleted embryos due to the deactivation of Myosin II.

The activity of Myosin II is upregulated by the phosphorylation of Myosin regulatory light chains, Myosin II activity regulators include Rho-associated, coiled-coil containing protein kinase (ROCK) and Myosin-light-chain kinase (MLCK), they regulate the $\mathrm{Ca}^{2+}$ sensitivity of Myosin II. ROCK belongs to Rho signaling pathway and it localizes to the upstream of Myosin II. The activity of ROCK is regulated by RhoA and its upstream protein RhoGEF2 (Kosako et al., 1999; Madaule et al., 1998). During cytokinesis, ROCK accumulates at the cleavage furrow, promotes the contractile ring formation by modulating F-actin-binding proteins such as ezrin/radixin/moesin (ERM) proteins and Myosin II. Furthermore, ROCK also can phosphorylate and regulate intermediate filaments proteins at the cleavage furrow (Kosako et al., 1999). MLCK activity is regulated by $\mathrm{Ca}^{2+}$ and its phosphorylation, the 
functions of MLCK in fibroblast and vascular contraction was conducted by regulating $\mathrm{Ca}^{2+}$ sensitivity of Myosin II (Kamm and Stull, 2001; Somlyo and Somlyo, 2003). Phosphorylation of Myosin II promotes the interaction between Myosin II and F-actin filaments, Myosin II filament formation is also enhanced by Myosin II phosphorylation (Scholey et al., 1980). Deactivation of Myosin II induces the decreasing of contraction force at the apical domain (Coravos and Martin, 2016). The result in this study could show that the disruption of Myosin II in Kinesin-1 RNAi embryos induces the shape change of F-actin caps.

Myosin II, as a motor protein, migrating along the actin filaments from the minus end to the plus end. The migration and accumulation of Myosin II to the intercap domain depend on the polarity of F-actin filaments. Live images and immunostainings of Myosin II could show that, during the interphase of syncytial blastoderms, the localization of Myosin II at the intercap domain was affected in Kinesin-1 RNAi embryos. The mislocalization of Myosin II in Kinesin-1 RNAi embryos leads to an assumption that the disruption of F-actin cap organization in Kinesin-1 RNAi embryos influences the migration of Myosin II toward to the edge of the cap domain, induces the defects of F-actin cap contraction. At the telophase of mitosis, before the formation of the F-actin cap, F-actin filaments are randomly distributed on the cortex of embryos. The Myosin II accumulation is not restricted to the intercap domain, the contractility force is not enough to induce the cap contraction, so the cap domain is diffused. However, with the F-actin cap organization, Myosin II localizes to the intercap domain, stronger contractility force induces the cap contraction and the size of the cap becomes smaller.

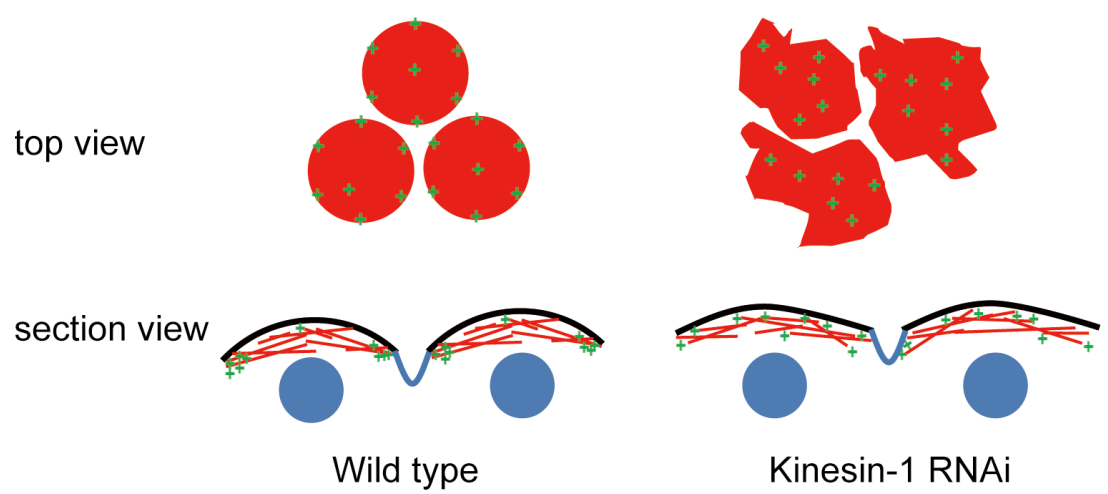

Figure 69 The mislocalization of $\mathrm{Cpa}$ affects cap domain contractility.

The distribution of F-actin (red circle and filament) and Cpa (green cross) in wild type and Kinesin-1 depleted embryos. The plus end of F-actin filaments mainly localizes to the intercap domain, this is essential for the function of Myosin II at the intercap domain, which improves the contraction of the Factin cap. In Kinesin-1 RNAi embryos, the disruption of Myosin II at the intercap domain influences the contraction of the F-actin cap and induces the shape change of the F-actin cap. 
The previous paper has reported that Myosin II remodels F-actin filaments in vitro (Wollrab et al., 2019). In vitro, Myosin II remodels random distribution of F-actin filaments to F-actin aster structures, Cpa localizes to the center of F-actin asters. The reorganization of F-actin filaments is due to the movement of Myosin II along Factinfilaments, which drives the high concentration of F-actin to form clusters. The publication provides evidence that Myosin II may also play a role in the F-actin cap organization. To test this assumption in Drosophila embryos, I injected Y-27632 into Cpa-GFP embryos and checked Cpa distribution. Y-27632 is a cell-permeable, highly potent and selective inhibitor of ROCK. Y-27632 inhibits both ROCK1 and ROCK2 by competing with ATP for binding to the catalytic site (Davies et al., 2000; Ishizaki et al., 1996). Myosin II localizes to the downstream of ROCK, Y-27632 significantly decreases Myosin II at the intercap region. The assumption could be supported by the result of Cpa-GFP distribution. The Cpa-GFP distribution is affected in embryos with Y-27632 injection, the Cpa-GFP accumulation at the intercap domain is affected in Kinesin-1 depleted embryos. The results in this study indicate the accumulation of Cpa clusters at the intercap domain is driven by Myosin II.

Functions of microtubules and F-actin in cell biology and biophysics have been studied for several decades, the interaction between microtubules and F-actin in core cellular processes has been concerned. Dia, as a member of Formin proteins, is required for the initiation of $\mathrm{F}$-actin nucleation. Previous publications indicated that Slam is essential for RhoGEF2 localization in Drosophila embryos and RhoGEF2 localizes to the upstream of Dia and regulates the activity of Dia (Großhans et al., 2005; Lecuit et al., 2002). Results I got from this study showed that Slam localization is regulated by Kinesin-1. Based on these, I hypothesized that Dia localizes to the downstream of Kinesin-1. The result supports this hypothesis, the localization of Dia is affected in Kinesin-1 depleted embryos. Dia localizes to the plus end of F-actin filaments and helps the elongation of F-actin filaments (Dominguez and Holmes, 2011; Pring et al., 2003; Rottner et al., 2010). Furthermore, microtubules can promote F-actin polymerization by recruiting CLIP-170 and mDia1 to the plus end of microtubules. The plus end accumulation of CLIP-170 and mDia1 can stimulate actin nucleation (Henty-Ridilla et al., 2016). As Dia is essential for F-actin polymerization, I checked the Cpa distribution in dia mutant. Results indicate that F-actin cap polarity is affected in dia mutant. In dia mutant, Cpa clusters mainly accumulate at the cap domain during syncytial and cellularization stages. 


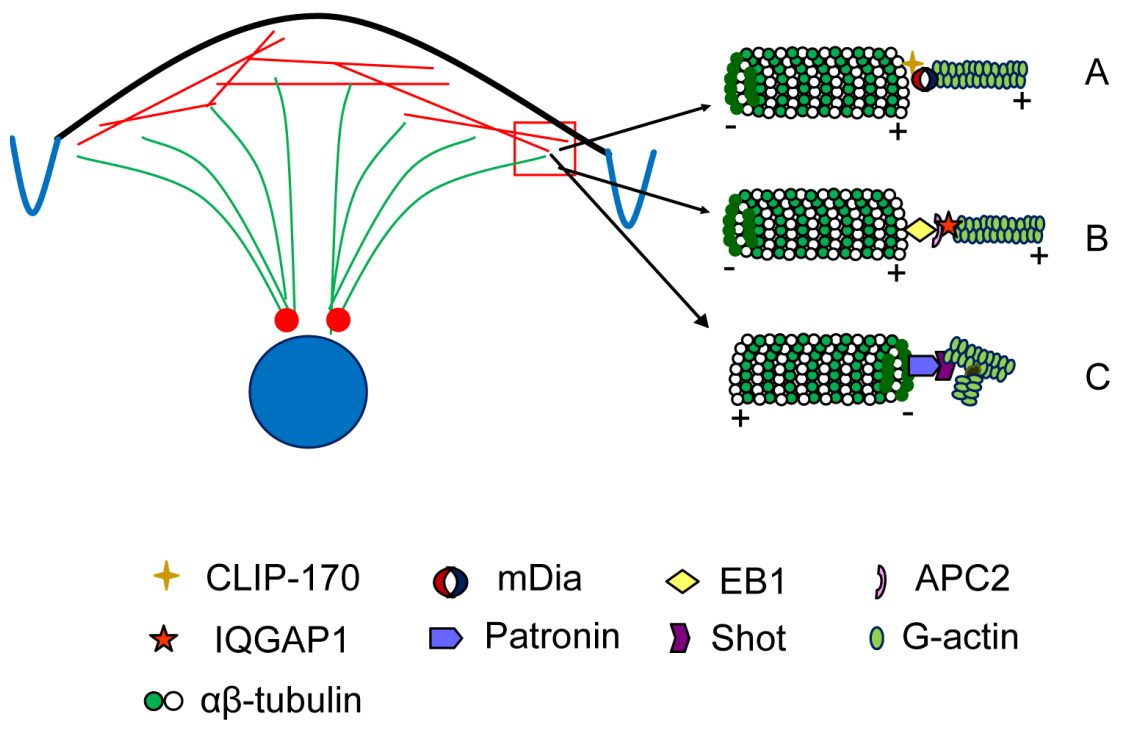

Figure $\mathbf{7 0}$ Interactions between microtubule and F-actin filament.

Previous reports have indicated that the connection between microtubule and F-actin filament via $(A)$ Recruiting CLIP-170 and mDia to the plus end of microtubules to form F-actin nucleation center (Perez et al., 1999). (B) Microtubule connects with F-actin via EB1/APC2/IQGAP1 complex (Juanes et al., 2019; Morrison, 2009, p. 1; Watanabe et al., 2004). (C) Shot inserts into the F-actin network and forms the nucleation center for microtubule with Patronin (Goodwin and Vale, 2010; Khanal et al., 2016).

Previous reports indicate $A P C$ protein has connections with both actin and microtubule. APC protein contains the $\beta$-catenin binding domain, it binds to $\beta$-catenin and localizes to the cell membrane. Besides, APC protein binds to the plus end of microtubules via EB1 and forms microtubule clusters (Munemitsu et al., 1994; Smith et al., 1994). Furthermore, in culture cell, IQGAP1, an effector of Cdc42 and Rac1, binds to APC protein, as a linker between microtubules and F-actin. The mutation of IQGAP1 or APC protein leads to the disruption of actin meshwork formation (Watanabe et al., 2004). Also, the F-actin localization is affected in APC2 mutant embryos (Webb et al., 2009). My results showed that APC2 localization is affected in Kinesin-1 RNAi embryos. Live images of embryos with Khc-Kate and APC-GFP indicate that these two proteins are localized to the cap domain during the interphase. The immunoprecipitation shows that Kinesin-1 and APC2 form complex in Drosophila embryos. Based on these results I assumed that APC protein is a key linker between F-actin cap polarity and Kinesin-1, the disruption of APC2 in Kinesin-1 RNAi embryos induces the mis-organization of F-actin cap and the mislocalization of cortical components.

To confirm the assumption, I first checked the cellularization in APC2 truncation embryos. Although the cellularization is affected in APC2 d40 embryos, the cortical polarization is not disrupted. Apical, subapical, lateral, basal domains are observed 
and different domains are clearly separated in APC2 d40 embryos. The results about APC2 protein indicating that although Kinesin-1 interacts with APC2, it is not the key factor for the disruption of cap domain polarity in Kinesin-1 RNAi embryos. As Slam and Amphiphysin at the basal domain are vital for membrane ingression during cellularization, one explanation about the disruption of cellularization is due to the decline of Slam and Amphiphysin at the basal domain in APC2 d40 embryos.

In this study, I investigated the function of Kinesin-1 in cortical differentiation and Factin cap polarity. Kinesin-1 is required for the membrane components localization and the cortical polarization in Drosophila early embryonic development. The F-actin cap contraction and Cpa distribution are affected in Kinesin-1 depleted embryos due to the mislocalization of Myosin II. Dia is a linker between microtubule and F-actin, the mislocalization of Dia in Kinesin-1 RNAi embryos induces to the mislocalization of Cpa. 
Part B Mapping of slam RNA sequence for Slam expression and RNA localization 


\section{Introduction: function and connection of slam}

\section{mRNA and protein in Drosophila early embryonic development}

slow as molasses (slam) gene can be found in Drosophila melanogaster, Slam protein, which is encoded by the slam gene, consists of 1155 amino acids, the expression of Slam can be detected in Drosophila embryos at stage 4, stage 5 and adult flies. Slam protein localizes to the basal domain during cellularization of Drosophila embryos, which not only is required for the furrow invagination during cellularization but also promotes the basal localization of slam mRNA. slam mRNA at the basal domain during cellularization is required for Slam local translation. Furthermore, it has been previously reported that the slam mRNA sequence not only contains information for Slam protein translation and but also contains information for slam mRNA basal localization (Yan et al., 2017; Yan and Großhans, 2018).

\subsection{Spatio-temporal regulation of proteins}

The subcellular localization of protein is crucial for its function, right localization of proteins depends on protein transporting, sorting, protein turnover, and physical barrier. For example, Bicoid acts as a transcription factor during Drosophila embryonic development (Fradin, 2017; Kugler and Lasko, 2009; Ling et al., 2019). Bicoid forms gradient along the anterior-posterior (A-P) polarity in Drosophila early embryonic development, the gradient of Bicoid regulates the transcription of multiple target genes. For example, Bicoid binds and regulates hunchback gene expression at the anterior part of embryos, where the concentration of Bicoid is higher than posterior. The proper activation of Hunchback protein expression is essential for the embryonic A-P polarity (Driever and Nüsslein-Volhard, 1989; Tautz, 1988). Embryos without Hunchback activity leads to the bicaudal phenotype (Simpson-Brose et al., 1994). Furthermore, Bicoid is important for head and thorax development (Lawrence, 1988).

In polarizing cell, proteins that localize to the specific domain are transported from the cytoplasm to membrane. Membrane components transporting depends on motor 
proteins such as Kinesin-1 and Dynein, the movement of these two proteins relies on microtubules (Terada, 2003; Terada et al., 2010). For example, motor protein dependent transport is important in neurons (Terada et al., 2010). As neurons have long axons and dendrites, they need a transport system to move synthesis proteins from the cell body to axons. There exists fast and slow axonal transport systems in neurons to transport cytosolic proteins and membranous proteins. These two transport systems are depending on Kinesin-1.

The right localization of membrane elements on cortical domains of cells depends on recycling endosomes and centrosomes (Acharya et al., 2014; Goldenring, 2015). Nuclear fallout (Nuf) is a marker protein of the recycling endosome. In the nuf mutant embryos, the localization of Rab11 is affected, it indicates that the recycling endosome is disrupted in the nuf mutant.

In addition to the protein localization, the amount of cortical proteins is also important for Drosophila early embryonic development. During gastrulation, the amount of Myosin II at the basal domain is crucial for the ventral furrow invagination (Krueger et al., 2018). In wild type embryos, before the ventral furrow formation starts, the basal domain accumulation of Myosin II is decreased, the amount of Myosin II at the apical domain rises. When the amount of Myosin II at the basal domain is increased, the membrane constriction is affected, this induces the failure of ventral furrow invagination.

\subsubsection{Localization and functions of Slam protein}

As a cortical component during Drosophila early embryonic development, Slam protein localizes to the intercap domain in syncytial blastoderms and it localizes to the basal domain during cellularization. Although Slam localizes to the new membrane rapidly after it forms during cellularization, Slam is not the initiator of the membrane ingression as shown previously by Acharya et al.. The previous publication showed that E-cadherin is present earlier at the newly formed membrane than Slam (Acharya et al., 2014). E-cadherin is encoded by the shortgun (shg) in Drosophila and it is required for adherens junction assembly (Golenkina et al., 2018; Haruta et al., 2010; lyer et al., 2019; Stehbens et al., 2006; Zhang et al., 2014).

Although Slam is not the protein that initiates the membrane invagination, Slam is essential for the membrane invagination during cellularization in Drosophila embryos (Acharya et al., 2014; Lecuit et al., 2002; Yan et al., 2017; Yan and Großhans, 2018). 
The Slam protein in Drosophila early embryos come from two resources: maternal expression and zygotic expression. The amount of Slam protein is important for the cellularization of Drosophila embryo, the furrow invagination is affected by either maternal or zygotic Slam protein depletion (Acharya et al., 2014). The previous publication showed that when the maternal and zygotic Slam protein were depleted in Drosophila embryos, the membrane invagination during cellularization was lost. When the maternal but not zygotic expression of Slam was depleted in Drosophila embryos, the furrow invagination was affected and the length of furrow was shorter than wild type during cellularization (Acharya et al., 2014)..

In addition to the function of Slam in cellularization, Slam is required for the germ cell migration (Stein et al., 2002). In Drosophila embryos, germ cells move from posterior pole to the midgut and then locate to the mesoderm during stage 9-11. Slam protein is required for this process. In slam zygotic mutant embryos, the translocation of germ cells through the midgut to mesoderm was affected, germ cell could be found in midgut or posterior of embryos.

Apart from functions of Slam during furrow invagination and germ cell migration, Slam also can regulate the localization of RhoGEF2, Myosin II and PatJ. For example, the amount of RhoGEF2 at the furrow canal is Slam dose-dependent (Wenzl et al., 2010), the amount and localization of Myosin II in Drosophila embryos depend on Slam (Lecuit et al., 2002).

In Drosophila embryos, multiple cellular processes depend on Slam. The amount and right localization of Slam protein are crucial for functions of Slam, Spatio-temporal dynamics of Slam are regulated in different ways. Dynamics of Slam protein are different in different stages. Slam shows high mobility at the onset of cellularization, whereas Slam has low mobility during the later stage of cellularization. The localization of Slam protein at the basal domain depends on the recycling endosome, the disruption of recycling endosomes induces the mislocalization of Slam protein in Drosophila embryos (Acharya et al., 2014). The result I got in this study shows that Kinesin-1 is also required for the Slam localization, the Slam protein is stuck at the surface of embryos in Kinesin-1 RNAi embryos (Figure 12 and 13). Furthermore, the APC2 protein is required for the Slam accumulation at the basal domain, the amount of Slam is reduced in APC2 truncation embryos (Figure 66). 


\subsection{The Spatio-temporal regulation and local translation of mRNA}

The subcellular localization of proteins is vital for their functions. Normally the localization of proteins is decided by the information contained in the protein sequence after translation, such as nuclear or mitochondrial localization sequences (Imai and Nakai, 2010). In recent years, more and more publications concern on mRNAs local translation (Aakalu et al., 2001; Cajigas et al., 2012; Yan et al., 2017). The local translation of mRNA is an evolutionarily conserved mechanism from yeast to highly differentiated cells such as neurons (Gonsalvez et al., 2005; Holt and Schuman, 2013). Local translation of mRNA is crucial for multiple cellular processes include growth cone, axonal responsiveness. The mRNA local translation is regulated by the interaction between mRNA and cytoskeleton, F-actin network provides a platform for mRNA translation, a set of translation factors and mRNA are associated with F-actin network (Hovland et al., 1996; Van Horck and Holt, 2008). Furthermore, the local translation of mRNA is also regulated by mRNA localization (Bi et al., 2007). mRNA local translation is well studied in neurons (Figure 71). Neurons serve as a very good model to illuminate mechanisms of the mRNA localization and the local translation, as they are highly polarized and the membrane front tip of the axon is far away from its cell body (Campenot and Eng, 2000; Taylor et al., 2009; Zivraj et al., 2010). mRNA local translation has some advantages. First, mRNA local translation provides a more flexible way for the regulation of protein localization, the localization information exists in the regulatory elements of mRNA 5' UTR or 3' UTR does not affect the function of mRNA, while if the localization information exists in the regulatory elements of protein, there has an effect on the function of protein (St Johnston, 2005). Second, the storage of mRNA to the functional region of cells is more efficient than storage of protein (Ellis, 2001), the responsiveness of mRNA storage local translation at the needed place is faster than protein translation and transporting to the function region (Ming et al., 2002; Piper et al., 2005).

Furthermore, axonal local translation also involved in retrograde signaling. For example, the injured axon synthesize importin $\beta$ locally, which transports nuclear localization signal (NLS) bearing proteins to the nucleus and influences the mRNA transcription and protein translation (Cox et al., 2008; Hanz et al., 2003).

Local translation can be found in many proteins (Feuge et al., 2019; Kashida et al., 2019; Manchalu et al., 2019). For example, Oskar is a posterior protein, which is 
required for the pole cell formation and posterior body structure. The transcription of oskar mRNA happens in nurse cells and oskar mRNA is transported to oocyte after transcription, oskar mRNA is translated in oocyte and Oskar protein localizes to the oocyte of Drosophila (Kim-Ha et al., 1991; Kugler and Lasko, 2009; Lehmann and Nüsslein-Volhard, 1986).

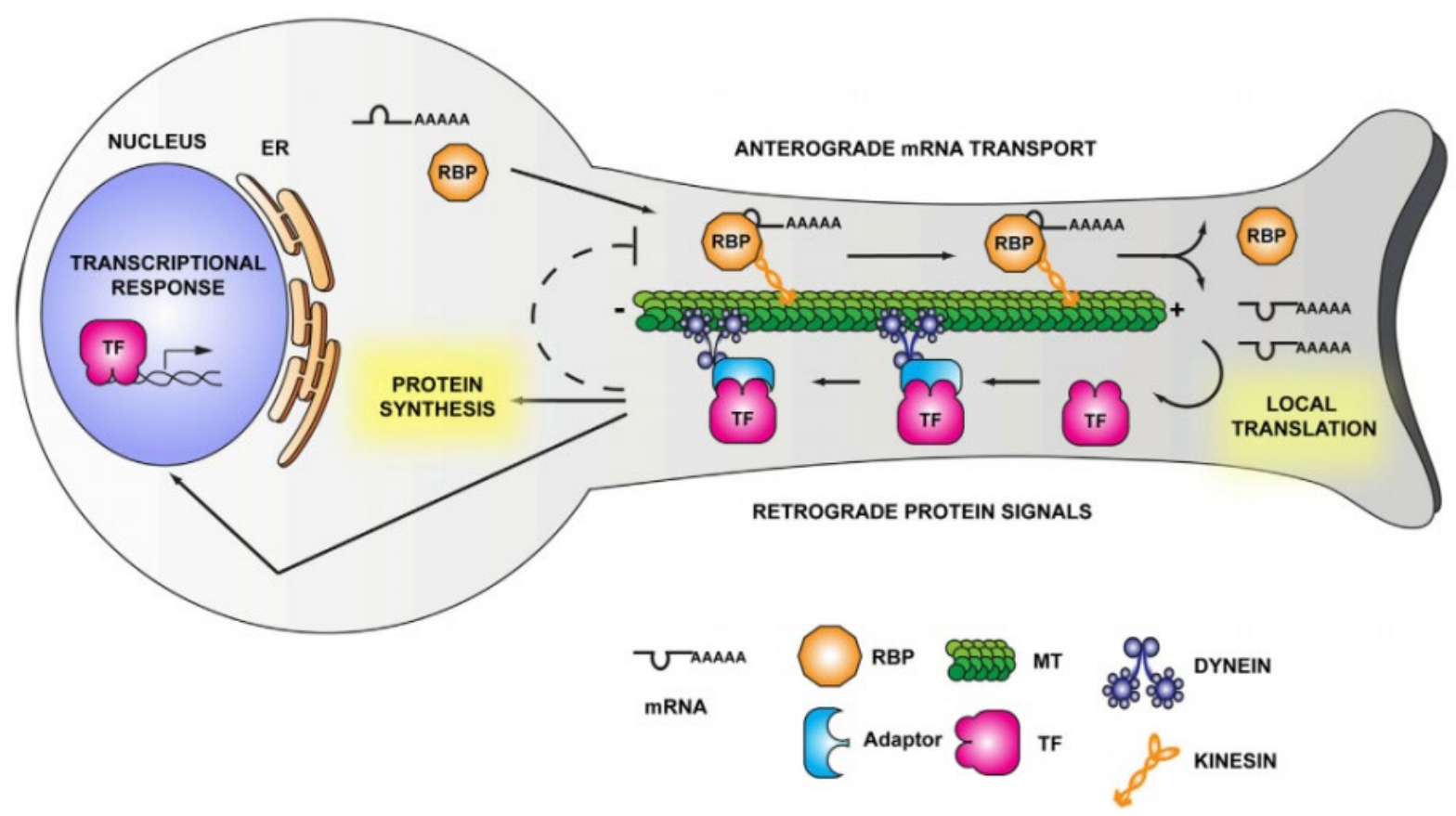

Figure 71 mRNA transport and local translation in neurons.

The schematic illustrates mechanisms of mRNA translocation, translation, protein retrograde transport between the cell body and axon of the neuron. Stimulation from axon induces a transcriptional response in the nucleus of neuron cell body, mRNA forms complex with their respective RNA binding protein (RBP). mRNA complex anterograde transport depends on Kinesin proteins moving along microtubules (MT) in the axon. The local translation of mRNA in axon can be induced by neuronal growth or injury. Translated proteins and transcription factors (TF) can be transported by Dynein to the cell body via binding to adaptor proteins. The dash line indicates a negative feedback loop. Adapted from M. Terenzio et al., 2017.

Translocation of oskar mRNA depends on elements such as microtubules, plus-end motors such as Kinesin-1 (Gáspár et al., 2017), minus-end motors such as Dynein and Staufen. Staufen protein is required for the localization and translational control of multiple maternal transcripts, therefore it is necessary for anterior and posterior body patterning (Ferrandon et al., 1994; Micklem et al., 2000; St Johnston et al., 1991). During the mRNA transporting, localization information in the sequence of localized mRNA is recognized by trans-acting factors, which is important for RNA transporting (Jambhekar et al., 2005; Meer et al., 2012). 


\subsubsection{Spatio-temporal dynamics and the local translation of slam mRNA}

In our lab, we focus on the interaction between slam mRNA and protein, also the impact of slam mRNA on Slam expression during early embryonic development (Yan et al., 2017). The previous paper has shown that slam mRNA and protein localize together at the tip of the furrow canal during cellularization. The right localization of slam mRNA and protein is vital for membrane ingression during the cellularization of Drosophila embryos. Yan et.al found that the Slam protein localizes to the basal domain during cellularization, and the protein can recruit slam mRNA to the basal domain, although there is apparent no RNA binding domain in Slam protein (Figure 72). Also we have not found that Slam protein shares any similarity to the known RNA binding protein, which suggests slam mRNA and protein might interact with each other indirectly.

By inserting bacteriophage PP7 hairpin loop near slam mRNA stop codon and expressing bacteriophage PP7 coat protein (PCP)-GFP marker protein, she found that at least part of slam transcripts are not fully translated before they arrive at the basal domain. Some slam transcripts arrive and finish translation at the basal domain (Yan et al., 2017).

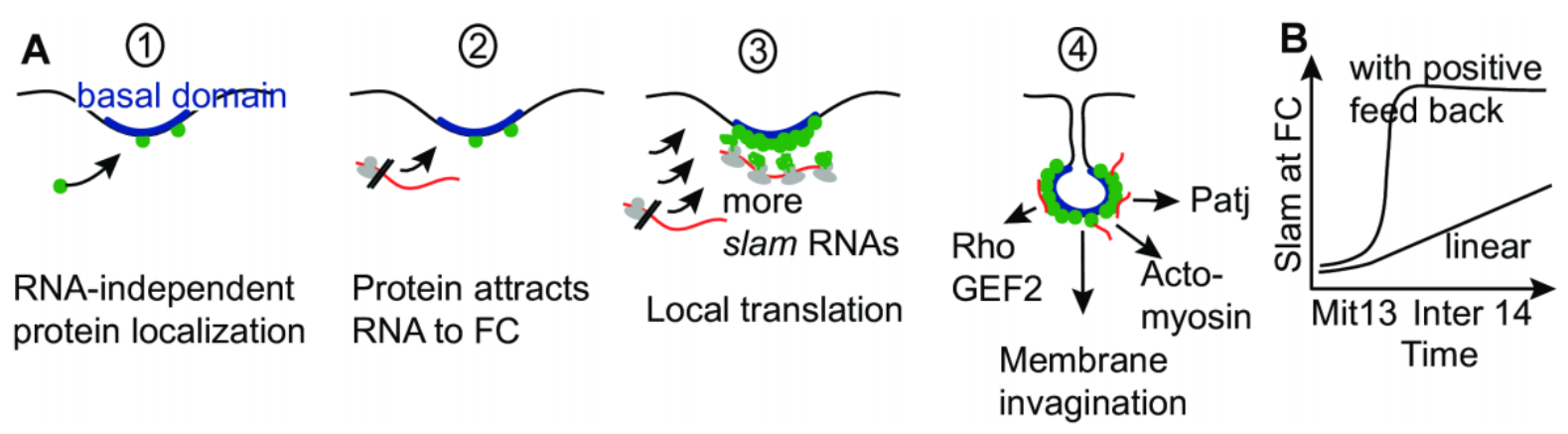

Figure 72 Schematic of Spatio-temporal dynamics of slam mRNA and protein.

(A) Processes of slam mRNA and protein accumulation at the basal domain during cellularization. (B) The amount of Slam protein at the furrow canal with positive feedback or according to a linear expression. Mit 13, mitosis of cell cycle 13; Inter 14, interphase of cell cycle 14. The figure was taken from Yan et al., 2017.

The amount of Slam protein varies a lot in Drosophila early embryonic development in different stages. Slam protein can be detected during the early syncytial stage. At this stage, the zygotic transcription of slam has not started yet, thus Slam protein is believed as the translation products from the maternal slam mRNA. The total amount of Slam protein increases continuously from the end of cell cycle 13 to the middle of cellularization. Furthermore, the amount of Slam at the basal domain increased 
robustly in early phase of cellularization. This is due to slam mRNA recruitment and the local translation of slam mRNA at the basal domain. The amount of Slam protein that localizes to the basal domain is quite stable throughout cellularization. However, slam mRNA concentration increases strongly at the beginning of cellularization and drops down after the mid of cellularization.

5.2.2 The function of slam mRNA sequence in slam mRNA localization and Slam protein expression

The codon sequence of slam mRNA contains information for Slam protein translation. The previous paper has reported that, when all the slam mRNA coding sequence is replaced with alternative codon usage (ACU), although there is no change for the sequence of amino acid in Slam protein, the Slam protein expression level decreased significantly and the cellularization is also disrupted (Yan et al., 2017; Yan and Großhans, 2018).

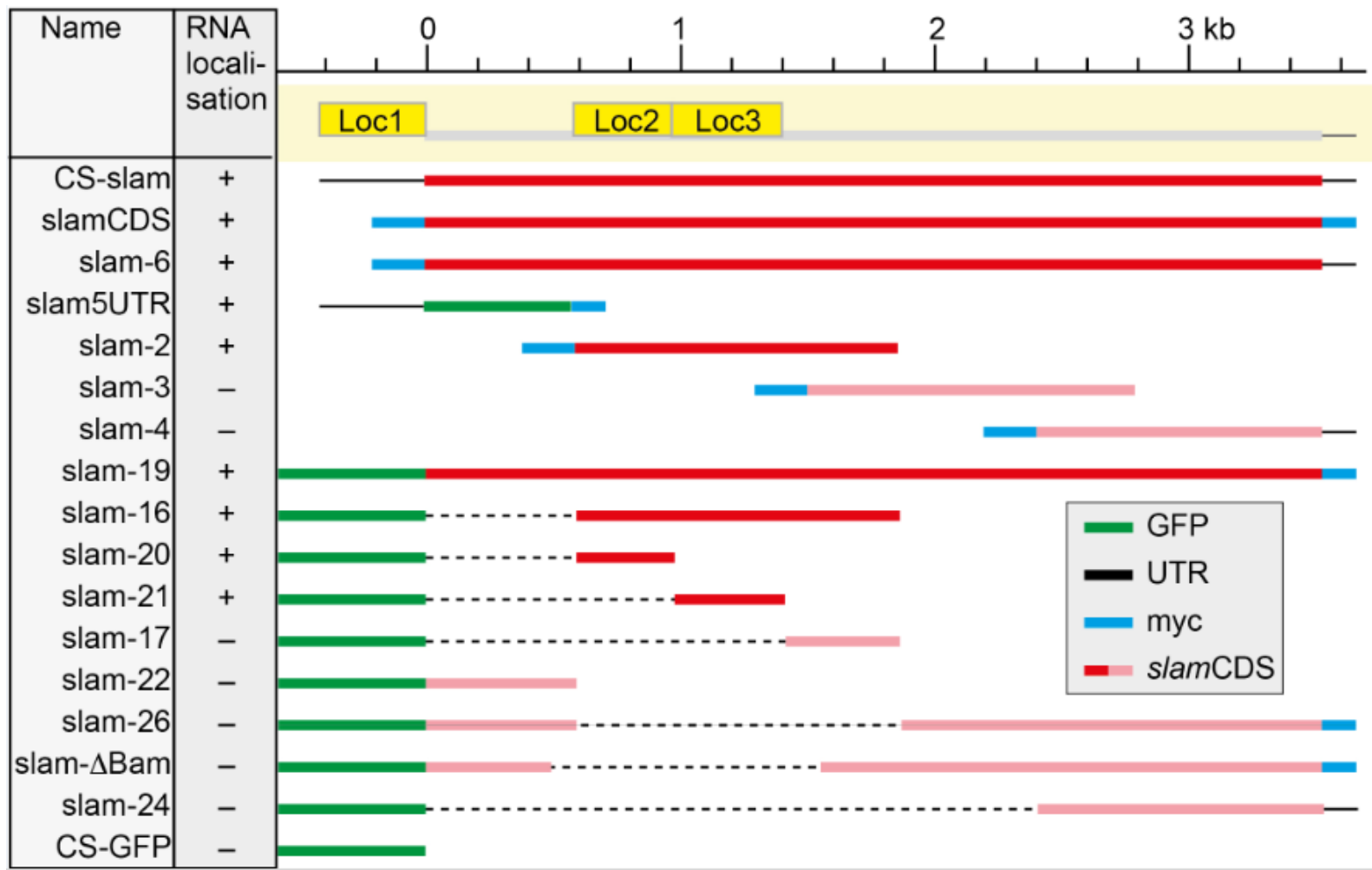

Figure 73 The function of different regions of the slam mRNA sequence in its localization.

The slam mRNA sequence is required for its localization at the basal domain during cellularization. There are three localization elements (Loc1, Loc2 and Loc3) in slam mRNA sequence, every single of them is sufficient for slam mRNA localization. When all three localization elements are deleted in Drosophila embryos, the basal accumulation of slam mRNA is affected. The figure is taken from Yan et al., 2017.

Different regions of slam mRNA for its localization have been identified. There are 
three localization elements in slam mRNA, localization element 1 (Loc1) localizes to the $5^{\prime}$ untranslated region (5' UTR), Loc2 and Loc3 localize to the coding region of slam mRNA (Figure 73). Loc1, Loc2 and Loc3 alone are sufficient for slam mRNA localization in Drosophila embryos during cellularization. Loc2 and Loc3 deletion can affect the localization of slam mRNA. Furthermore, when the whole slam mRNA sequence is replaced with ACU, the slam mRNA loses the accumulation at the basal domain during cellularization. These results indicate that the slam mRNA sequence is important for the RNA/protein localization, and the RNA localization is required for the translation of Slam protein at the basal domain.

With previous results about slam ACU we got the knowledge that slam mRNA regulates Spatio-temporal translation of Slam protein, but the specific regions of slam mRNA required for the slam mRNA localization and the Slam protein expression respectively have not been defined. In this study, I mapped the regions of slam mRNA. I replaced different parts of slam mRNA with synonymous codons and checked if there was an effect on the slam RNA/protein localization, protein expression level, and the physiological function, in terms of membrane invagination during cellularization. I found that the full length of wild type slam mRNA is required for the robust protein expression and membrane ingression, and the segments from $2818 \mathrm{nt}$ to $3522 \mathrm{nt}$ and $685 \mathrm{nt}$ to $1576 \mathrm{nt}$ is necessary for the localization of slam mRNA. 


\section{Results}

\subsection{Noncoding functions of slam mRNA for RNA localization and translation}

In Drosophila embryos, during cellularization, slam mRNA and Slam protein localize to the basal domain, which is required for the membrane invagination (Yan et al., 2017). To confirm the function of slam mRNA in the membrane invagination during cellularization, the DIC microscopy was used to check the cellularization in wild type embryos and slam_fl_acu germline clone embryos (referred to hereafter as slam_fl_acu). The result showed that when the slam mRNA was replaced by synonymous codons, the membrane invagination was affected, the furrow length in slam_fl_acu embryos was shorter than wild type (Figure 74). The result indicated that the slam mRNA sequence was required for membrane invagination.

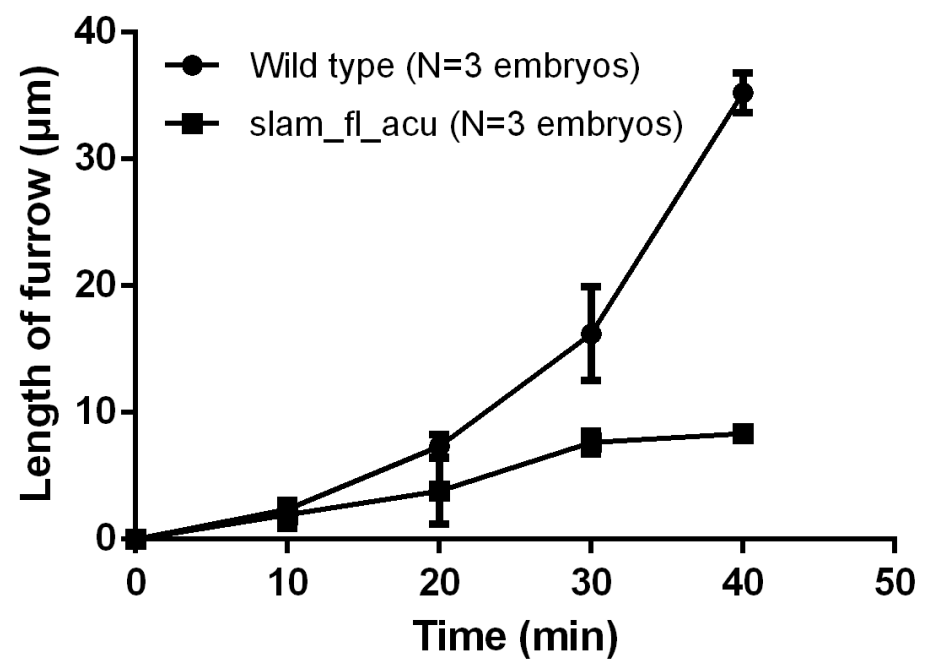

Figure 74 The membrane invagination is affected in slam_fl_acu embryos.

The membrane invagination in wild type embryos and slam_fl_acu germline clone embryos during cellularization. Spots represent furrow lengths in wild type and slam_fl_acu germline clone embryos at the indicated time points. Error bars represent s.e.m.

Previous publications have reported that the expression of Slam protein is decreased due to the slam mRNA sequence replaced with synonymous codons (Yan et al., 2017; Yan and Großhans, 2018). To confirm this, I checked Slam expression level in my study. The western blot showed that Slam expression was higher in wild type embryos than slam_fl_acu embryos. This result indicated that the slam mRNA 
sequence was vital for Slam protein expression (Figure 75).

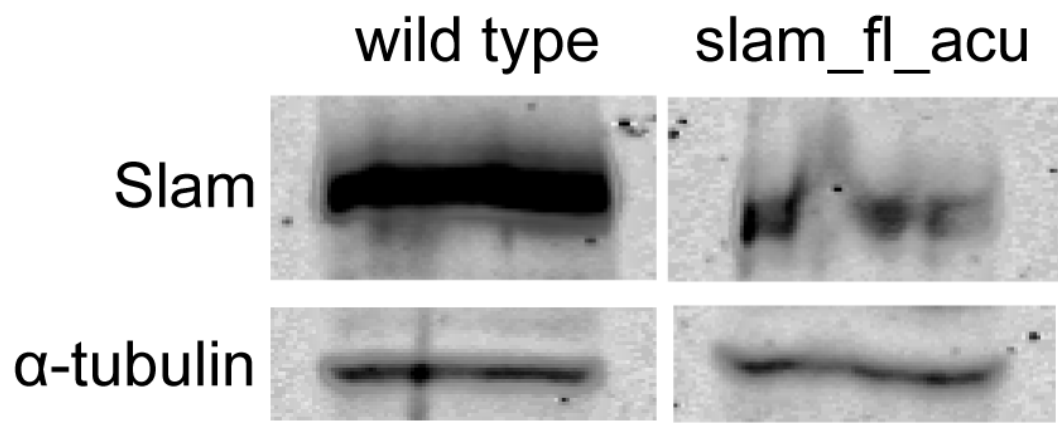

Figure 75 The Slam protein expression is decreased in slam_fl_acu embryos during cellularization.

The result shows the expression level of Slam in wild type and slam_fl_acu germline clone embryos. There are 20 embryos for each sample, the embryos are stained with DAPI, embryos in the cellularization stage are sought and used for western blot.

slam mRNA not only influenced Slam protein translation but also it was required for its own localization at the basal domain during cellularization (Figure 76). In wild type embryos, slam mRNA and protein localize to the basal domain during cellularization. However, in embryos (slam_fl_acu germline clone embryos) which genomic expressed slam ACU mRNA, the localization of slam ACU mRNA was affected, the majorty of slam ACU mRNA localized to the apical domain, only a small part of slam ACU mRNA localized to the basal domain. It indicated that the slam mRNA sequence was essential for its own localization.

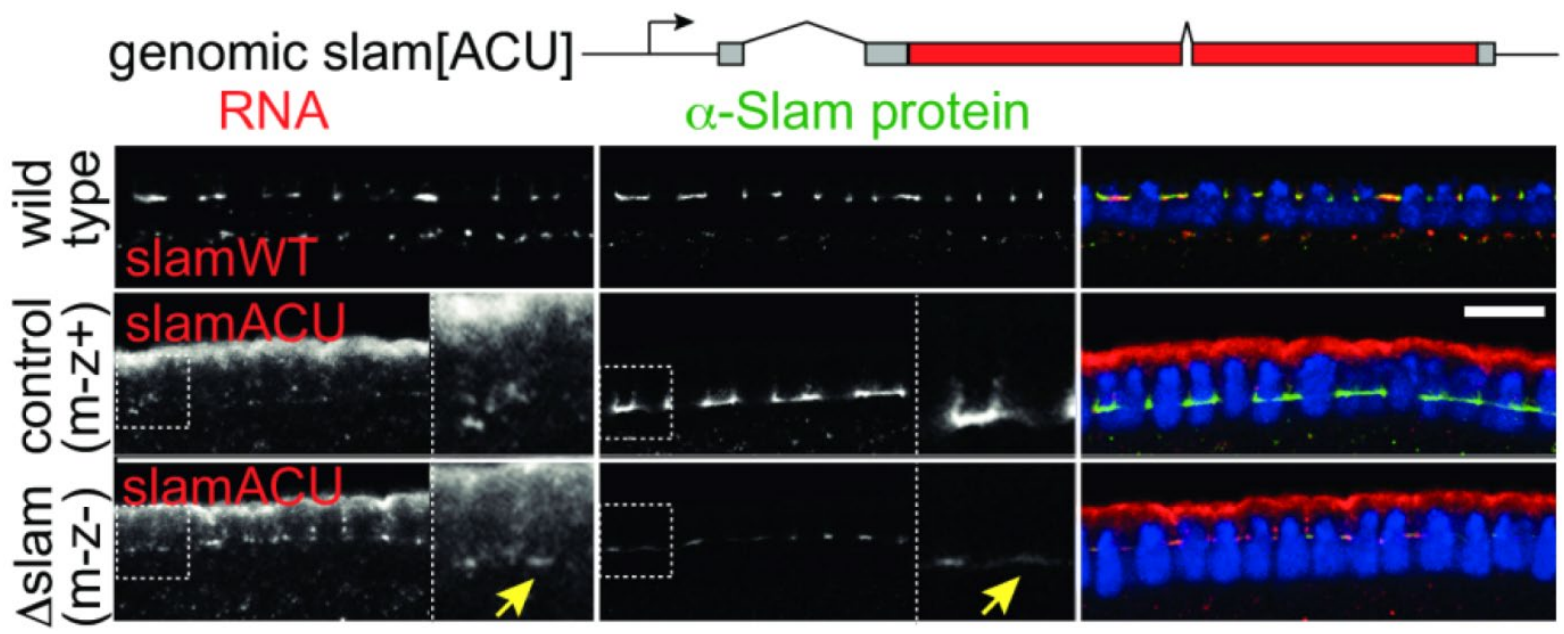

Figure 76 slam mRNA sequence is required for its basal localization during cellularization.

The result shows the localization of slam mRNA in wild type embryos and the localization of genomic transcription of slam ACU mRNA in zygotically rescued (m-z+) and slam deficient embryos (m-z-) from slam germline clones (slam_fl_acu germline clone embryos). Yellow arrows indicate slam mRNA and protein localization. Scale bar: $10 \mu \mathrm{m}$. The figure is taken from Yan et al. (2017).

To better understand the role about different segments of slam mRNA in the Slam 
protein expression and the membrane invagination, I designed three different hybrid sequences of slam mRNA (slam_acu $)$.

\subsection{Mapping of the localization and translation elements in slam mRNA}

It has been reported that the slow as molasses (slam) mRNA and its protein colocalizes to the furrow canal in Drosophila embryos during cellularization. slam mRNA and protein are essential for the membrane invagination and the basal domain formation (Acharya et al., 2014; Wenzl et al., 2010; Yan et al., 2017). Changing slam mRNA sequence with ACU can significantly affect the slam mRNA localization and decrease Slam protein expression. The slam mRNA sequence is required for both slam mRNA localization and Slam protein expression. However, it is not clear which part of slam mRNA is vital for the slam mRNA localization and which part of slam mRNA is vital for the Slam expression. For this purpose, different hybrid sequences of slam mRNA, in which different parts of slam mRNA were replaced with ACU, were designed to explore the region of slam mRNA essential for slam mRNA localization or Slam protein expression (Figure 77). In contrast to the previous RNA localization mapping, my experiment was conducted with genomic transgenes to avoid the effect of expression timing and location.

Previous results from our lab have indicated that when the segment of slam mRNA coding region from 507 nt to 1576 nt was replaced with synonymous codons (BamH_acu), there was no significant change for the slam mRNA localization and Slam protein expression. Three hybrid sequences of slam mRNA were designed: slam_acu1, replaced slam mRNA coding region with synonymous codons from $1 \mathrm{nt}$ to $1576 \mathrm{nt}$; slam_acu2, replaced slam mRNA coding region with synonymous codons from $507 \mathrm{nt}$ to $3522 \mathrm{nt}$; slam_acu3, replaced slam mRNA coding region with synonymous codons from $507 \mathrm{nt}$ to $2818 \mathrm{nt}$.

The result from DIC microscopy showed that embryos displayed defective membrane invagination during cellularization when the full slam mRNA coding sequence were replaced with synonymous codons (Figure 74). In my study, the result showed that not only in slam_fl_acu but also in slam_acu1, slam_acu2 and slam_acu3 embryos, the cellularization was affected, the furrow ingression was slower compared to wild type (Figure 78). The time when the membrane invagination started in different 
embryos was defined as 0 min.

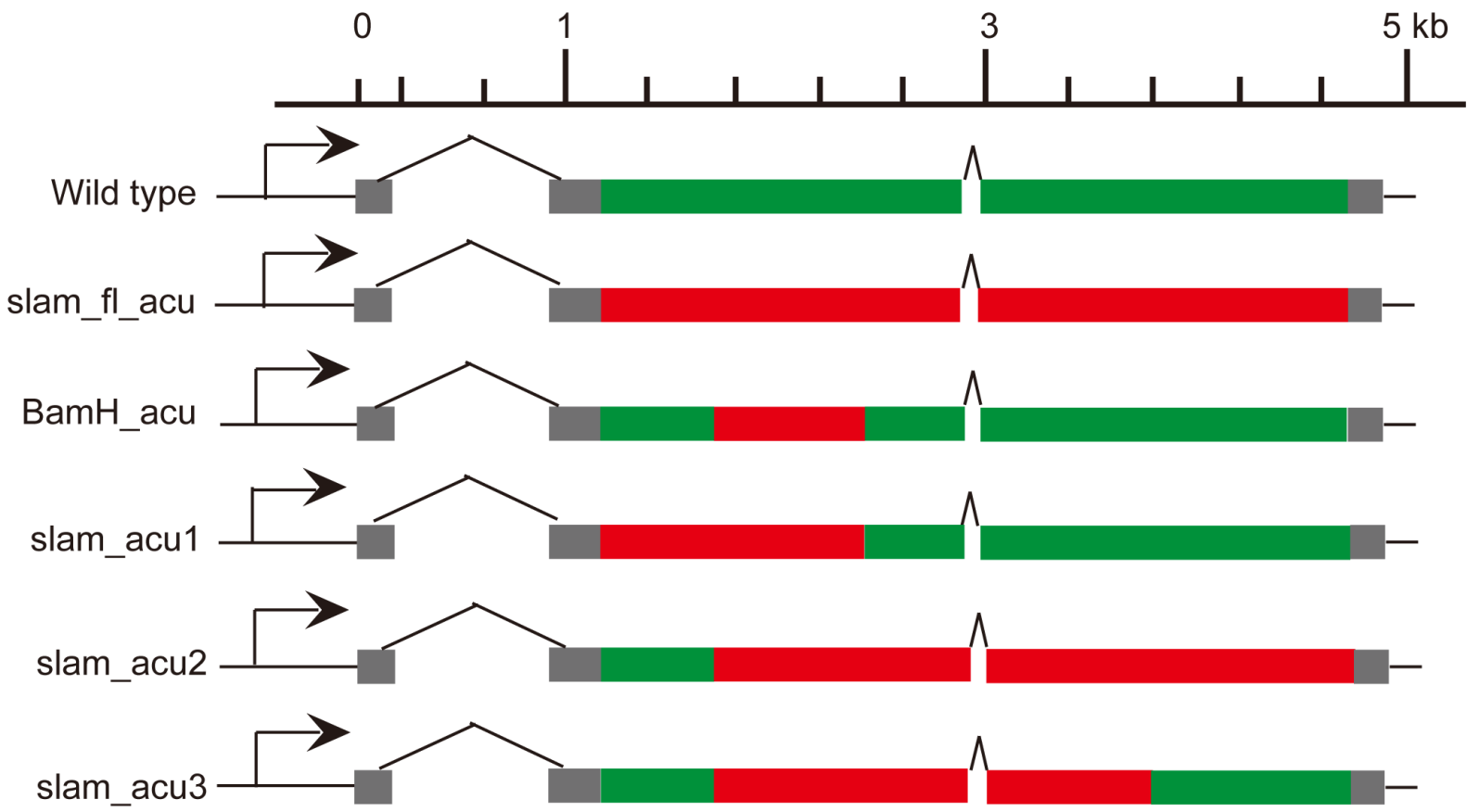

Figure 77 Different sequences of slam mRNA.

slam_fl_acu, replaces the whole slam mRNA coding region with synonymous codons; BamH_acu, replaces slam mRNA coding region from 507 nt to 1576 nt with synonymous codons; slam_acu1, replaces slam mRNA coding region with synonymous codons from $1 \mathrm{nt}$ to $1576 \mathrm{nt}$; slam_acu2, replaces slam mRNA coding region with synonymous codons from $507 \mathrm{nt}$ to $3522 \mathrm{nt}$; slam_acu3, replaces slam mRNA coding region with synonymous codons from $507 \mathrm{nt}$ to $2818 \mathrm{nt}$.

To better understand the importance of the slam mRNA sequence during cellularization, the furrow length of embryos with different hybrid slam mRNA sequences were measured at the later stage of cellularization (40 min). The quantification showed that, the furrow length was significantly different between wild type and embryos with different hybrid slam mRNA sequences in 40 min (Figure 79). The furrow length in wild type was about $30 \mu \mathrm{m}$. However, there was a big variation in embryos with different hybrid slam mRNA sequences, the furrow length in most embryos was significantly shorter compared to wild type embryos. Furthermore, although the furrow length in slam_acu1 embryos was diverse, there was no significant difference compared to the wild type. The result indicated the slam mRNA sequence was required for membrane invagination.

\subsection{The slam mRNA sequence is required for Slam expression}

slam mRNA and protein localize to the furrow tip and they are essential for basal domain specification, the amount of Slam protein at the basal domain increased 
dramatically during cellularization due to the local translation of slam mRNA (Yan et al., 2017).

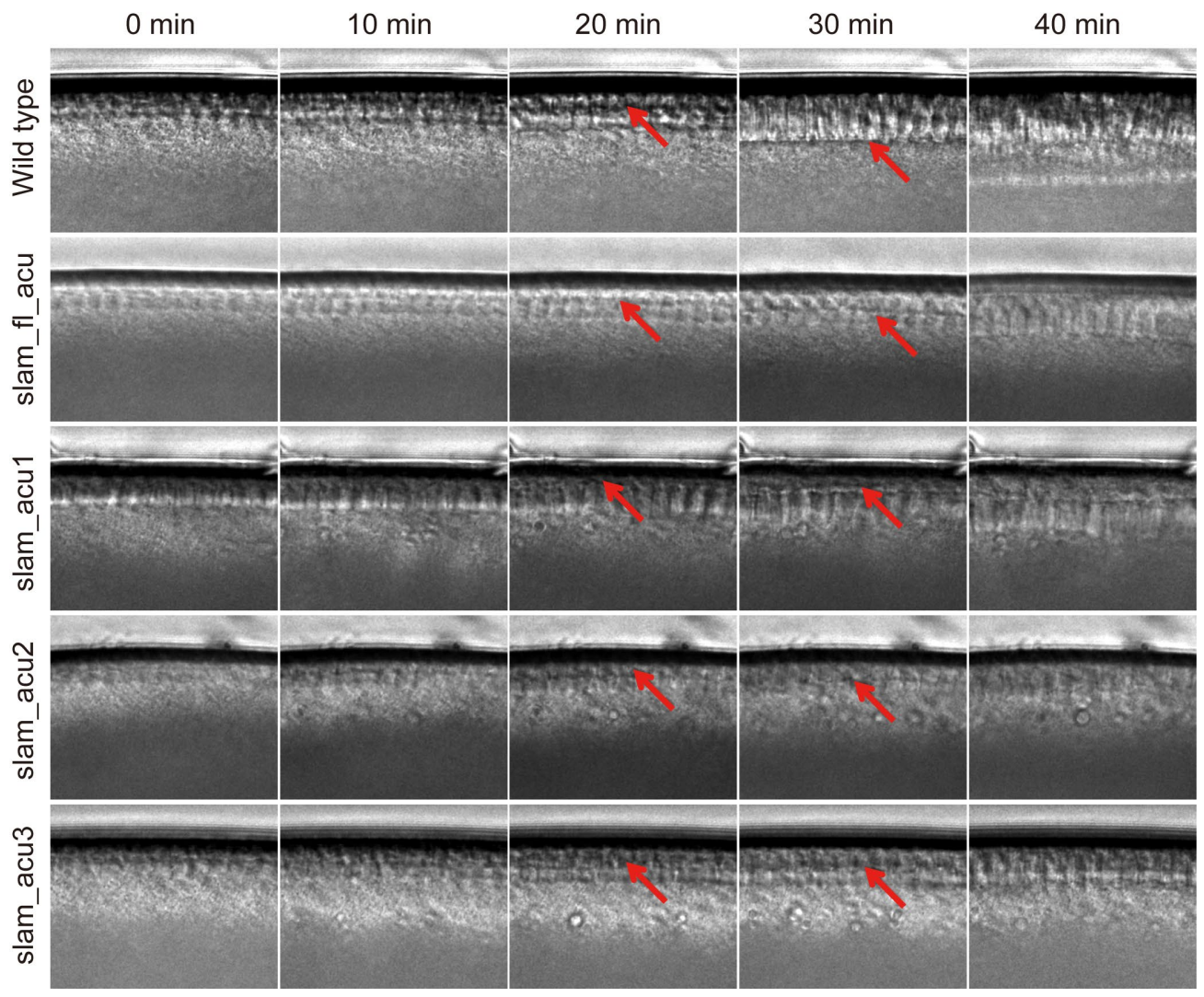

Figure 78 The slam mRNA sequence is required for the membrane invagination.

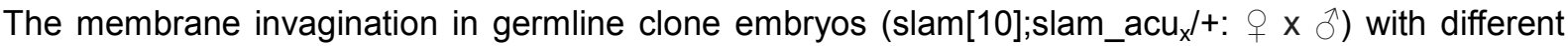
constructs of slam ACU during cellularization. The red arrows indicate fronts of the membrane in embryos at different time points. The size of the pictures is $73.5 \times 59.5 \mu \mathrm{m}$.

Previous results in this study have shown that the furrow invagination was affected in embryos with different sequences of slam ACU compared to wild type (Figure 78 and 79). Furthermore, the previous publication showed that Slam expression is decreased in slam_fl_acu embryos (Yan et al., 2017).

These results indicated that failures of cellularization in embryos with different hybrid slam ACU sequences may due to the decline of Slam expression. To confirm this hypothesis, Slam expression levels in embryos with different hybrid sequences of slam mRNA were checked by western blot (Figure 80). The result showed that compared to the wild type, Slam expression declined in embryos with different sequences of slam mRNA.

Slam expression level changes dramatically during the onset of cellularization. The amount of Slam protein at the basal domain is low at the beginning of cellularization and the Slam expression increases sharply during cellularization, the amount of Slam 
reaches to the highest point in about 15 min from the onset of cellularization (Yan et al., 2017).

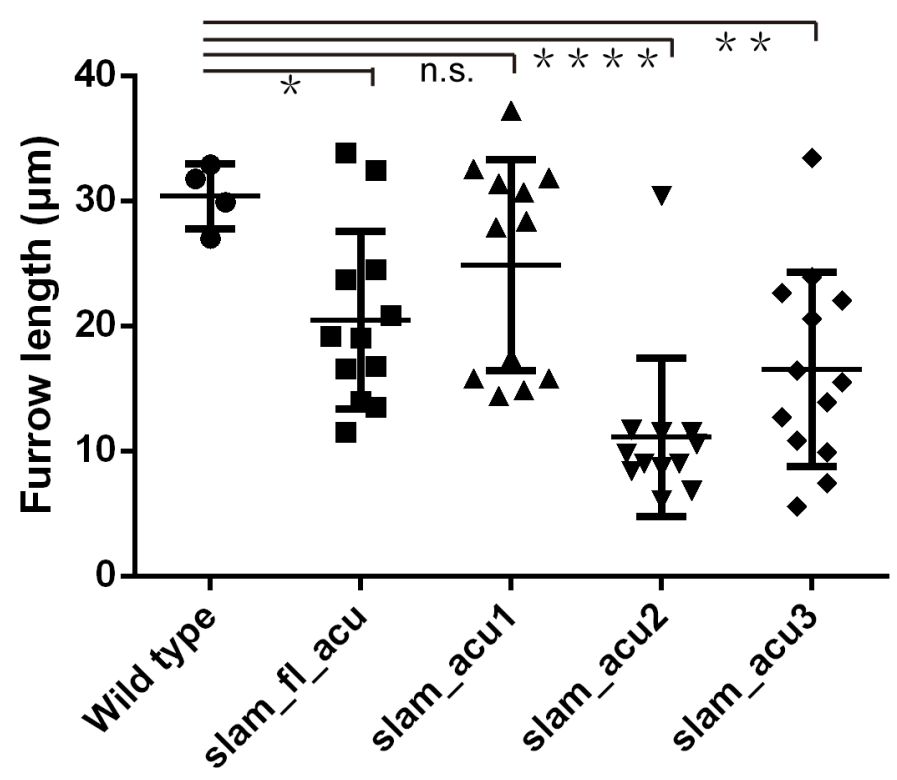

Figure 79 The membrane invagination is affected in embryos with different hybrid sequences of slam mRNA.

The result shows the length of furrow in wild type and embryos with different hybrid slam mRNA sequences during the later stage of cellularization (in $40 \mathrm{~min}$ after membrane invagination started). Spots represent furrow lengths in different embryos ( 5 furrows were measured for each embryo). Error bars represent s.e.m. n.s., no significance; *, p<0.05; **, p< 0.01; ****, $p<0.0001$.

Western blot reflects the total amount of Slam protein in multiple embryos, it cannot tell us the amount of Slam at the basal domain in every single embryo. To accurately describe the amount of Slam at the furrow canal in single embryo, I stained Slam protein in wild type embryos and embryos with different hybrid slam mRNA sequences.

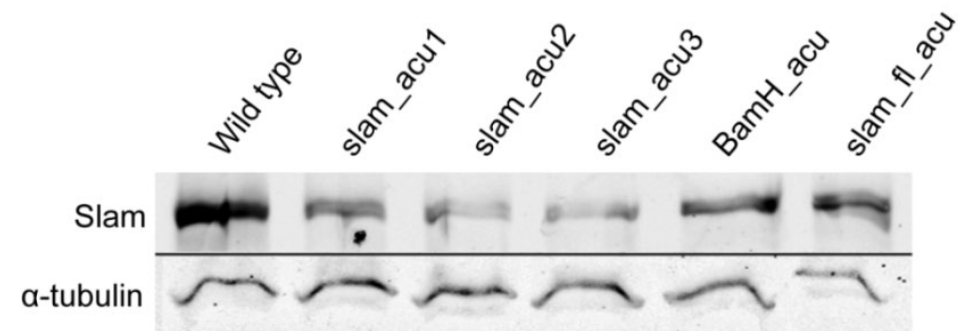

Figure 80 The decreasing of Slam expression in embryos with different hybrid slam mRNA sequences.

The result shows Slam expression in germline clone embryos with different slam ACU sequences during cellularization. Embryos with different hybrid slam mRNA sequences are selected by DAPI staining, embryos which are in the middle of cellularization are sought out under the fluorescent microscope. 20 embryos are used for each sample. 
All stainings were performed with His-GFP embryos in the same tube respectively. Fluorescent intensities of Slam protein in His-GFP embryos, wild type embryos and embryos with different hybrid slam mRNA sequences were acquired under the same settings (Figure 81).

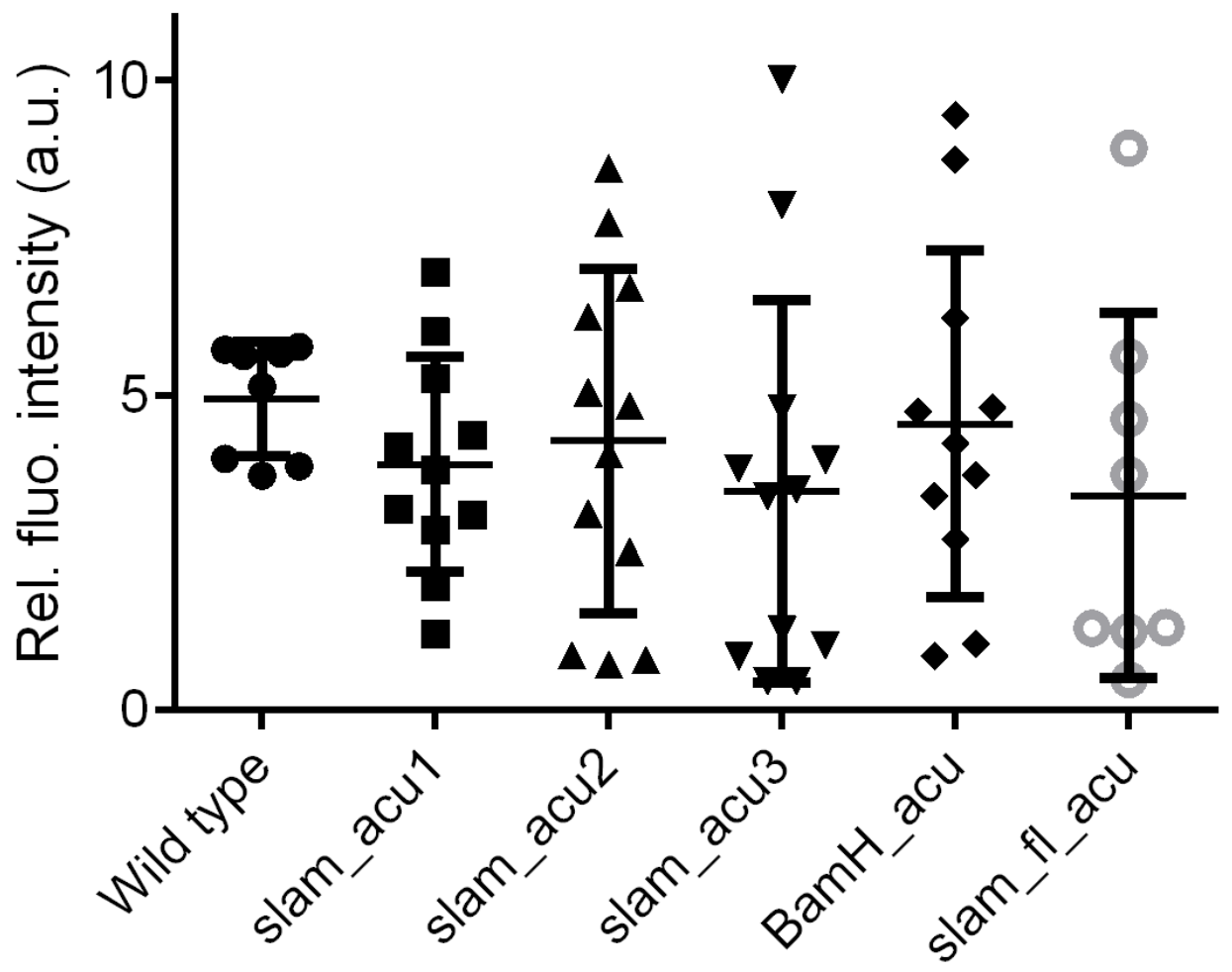

Figure 81 Different hybrid slam mRNA sequences induce various amount of Slam at the basal domain.

The amount of Slam was detected by measuring Slam fluorescent intensity at the basal domain and compare it to the Slam fluorescent intensity in His-GFP embryos in the same tube. For fluorescent intensity of Slam protein at the furrow tip during cellularization, there is no statistic significance between wild type and embryos with different slam ACU constructs. Error bars represent s.e.m.

The quantification of the fluorescent intensity in wild type and the embryos with different hybrid slam mRNA sequences showed that, for the amount of Slam protein at furrow canal, the embryo-to-embryo variation was small in wild type embryos. Whereas the variation was larger in embryos with different hybrid slam mRNA sequences (Figure 81). For example, the average amount of the Slam protein at furrow canals in slam_acu1 embryos was similar to wild type, strikingly, the Slam fluorescent intensity at the basal domain in different embryos varied in a larger region. The result indicated that the slam mRNA sequence was required for the robustness Slam accumulation at the basal domain. 


\subsection{The slam mRNA sequence is required for slam mRNA}

\section{localization}

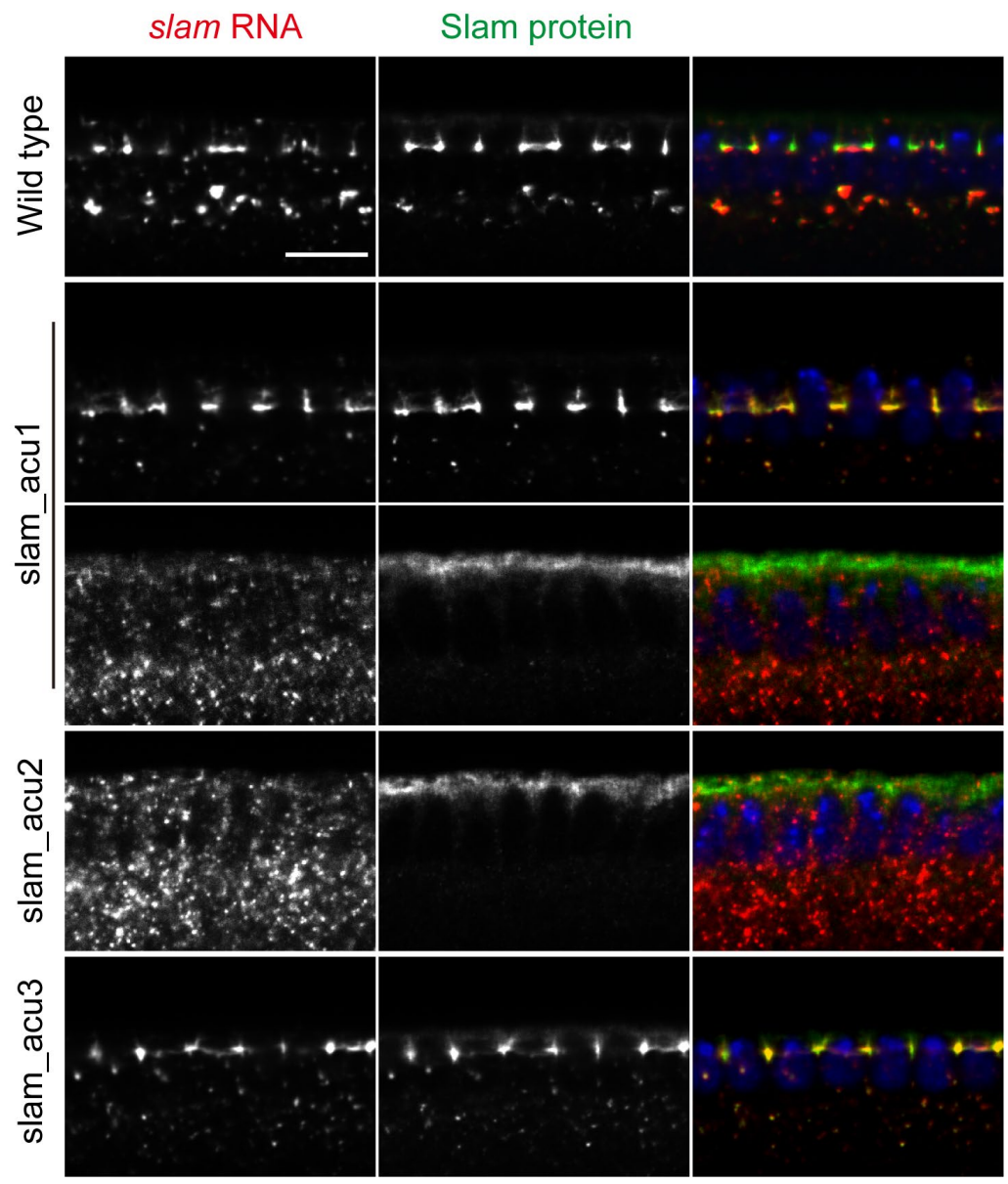

Figure 82 The slam mRNA sequence is required for the localization of slam mRNA and protein. The result shows the in situ hybridization of slam mRNA (red) and immunostaining of Slam protein (green) in germline clone embryos with different hybrid slam mRNA sequences. The result shows the slam mRNA sequence is required for slam mRNA and Slam protein localization. Scale bar:10 $\mu \mathrm{m}$.

It has been reported that the slam mRNA sequence is required for slam mRNA localization (Yan et al., 2017). Here I checked whether changing part of the slam mRNA sequence with synonymous codons affects the slam mRNA localization as well.

The result showed that slam mRNA and protein colocalized to the basal domain in wild type (Figure 82). However, slam mRNA and protein mislocalized to the cortex in slam_acu1 and slam_acu2 embryos. Surprisingly, slam mRNA and protein were localized to tip of the furrow properly in slam_acu3 embryos.

Taken together, results that I got in this study indicated that the slam mRNA sequence was important for cellularization in Drosophila embryos, the slam mRNA 
sequence was required for Slam protein expression and slam mRNA localization.

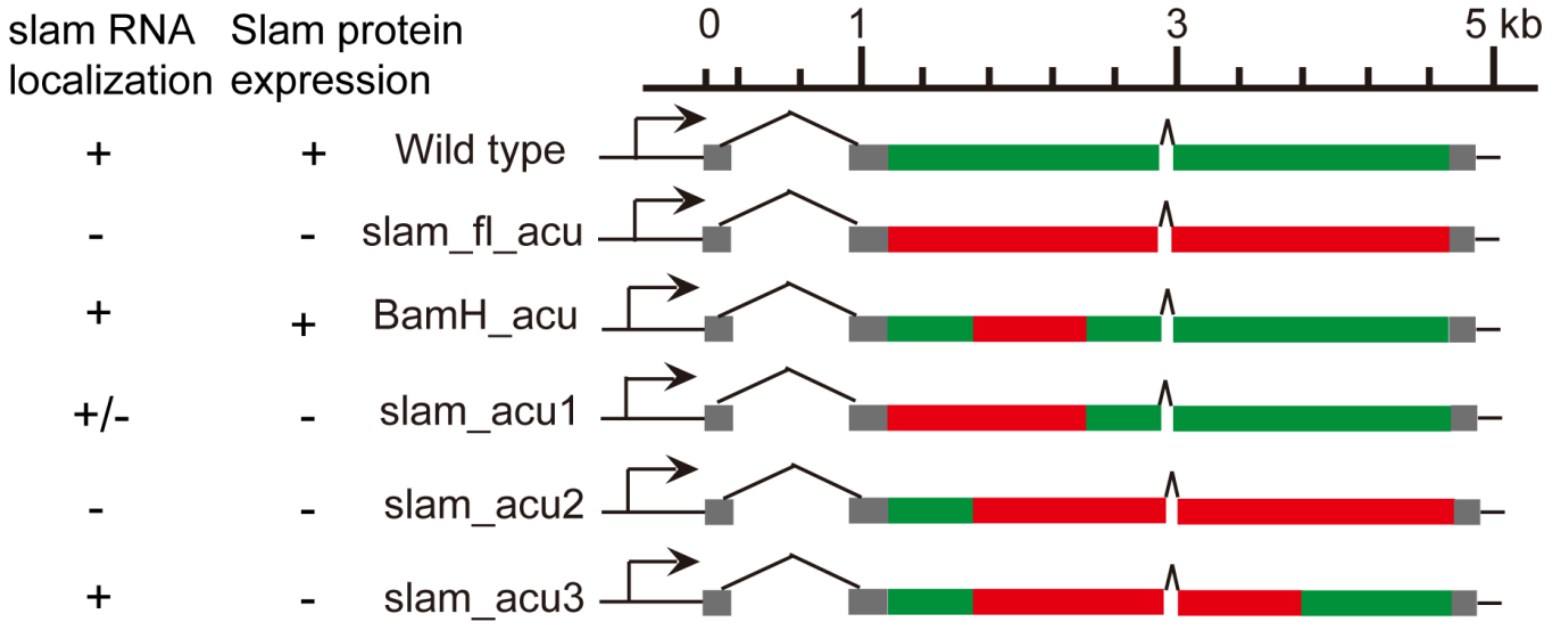

Figure 83 slam mRNA localization and Slam protein expression level in wild type embryos and embryos with different slam ACU constructs.

$(+)$, no effect; (-), have an effect; (+/-), part of embryos are affected.

Furthermore, I also found that different parts of slam mRNA have different influence to Slam protein expression and slam mRNA localization, C terminal of slam mRNA (from $2818 \mathrm{nt}$ to $3522 \mathrm{nt}$ ) was required for Slam protein expression, but not for slam mRNA and protein localization (Figure 82). 


\section{Discussion}

The study aimed to analyze different segments of the slam mRNA sequence essential for the slam mRNA localization or Slam protein translation in Drosophila embryos. In Drosophila embryos, Slam protein localizes to the furrow tip during the mitosis of syncytial blastoderms and cellularization. The right localization of Slam protein at the basal domain during cellularization is essential for membrane ingression (Acharya et al., 2014; Wenzl et al., 2010; Yan et al., 2017). In addition to the function of Slam in membrane invagination during cellularization, at the later stage, Slam protein is also required for the germ cell migration (Stein et al., 2002). The previous publication has mapped the function of the $5^{\prime}$ untranslated region ( $5^{\prime}$ UTR) and the coding sequence of slam mRNA for its localization (Yan et al., 2017). The result indicated that multiple segments of the slam mRNA that are sufficient for slam mRNA localization, slam mRNA sequences with every single localization element are sufficient for slam mRNA localization. Moreover, embryos replaced the whole slam mRNA coding sequence with synonymous codons (slam_fl_acu), the slam mRNA lost the ability to localize to the furrow canal, the Slam protein expression efficiency is decreased as well. As a result, the amount of Slam protein at the basal domain in slam_fl_acu embryos is lower than wild type. Furthermore, different regions of slam mRNA replaced with synonymous codons and expressed in cultured Drosophila S2 cells have proved that the region at the N-terminal (aa 164- aa 532) of slam mRNA coding sequence is essential for slam ACU truncation proteins localization (Yan and Großhans, 2018). Results from the previous paper indicated that the slam mRNA sequence contains information not only for Slam translation but also for slam mRNA localization.

However, slam mRNA mapping results in previous publications obtained by injecting slam mRNA into wild type embryos or transfecting slam ACU into Drosophila S2 cells (Yan et al., 2017; Yan and Großhans, 2018), there was no full length of slam mRNA existed. There is no experiment to explain the function of specific regions of slam mRNA in its localization and the translation efficiency of slam mRNA in intact slam mRNA and Slam protein background. In this study, I generated three different hybrid slam mRNA sequences (slam_acu, slam_acu 2 and slam_acu ${ }_{3}$ ), in which different parts of the slam mRNA coding sequence were replaced with synonymous codons. 
Embryos with different hybrid slam mRNA sequences transcripts intact slam mRNA and express intact Slam protein. The results I got in this study indicate that during cellularization, the membrane ingression was affected in three different hybrid slam mRNA fly lines, especially in slam_acu2.

Although the slam mRNA sequence in all different hybrid slam mRNA embryos is entire, the total amount of Slam protein in embryos with different slam mRNA sequences during cellularization is decreased (Figure 74). This may due to the codon bias (Sørensen and Pedersen, 1991), the uneven use of synonymous codons during protein translation. Original slam mRNA sequence partly or fully replaced by synonymous codons, which may induce the changing of slam translation efficiency and mRNA stability. Due to the codon degeneracy (61 codons encode 20 amino acids), multiple codons encode the same amino acid during protein translation. However, the ribosome recognition efficiency and tRNA concentration for synonymous codons are variable, these differences affect the velocity of protein translation elongation. For some codons with less abundant of tRNA, the ribosome has to wait longer time for the tRNA enter to the A-site of the ribosome, while ribosomes spend less time for optimal codons. The previous publication has reported that three Firefly luciferase (Luc) mRNAs (optimal codons according to the codon usage table, wild type, and least preferred codon used for all amino acids respectively) were designed, the time points of the first appearance of the luminescence signal were about $7.0 \mathrm{~min}, 8.4 \mathrm{~min}$, and $13.1 \mathrm{~min}$ respectively, this indicates that the elongation rate is affected by different codon usages ( $\mathrm{Yu}$ et al., 2015).

In addition to codon bias, mRNA stability is also crucial for protein translation. The stability of mRNA is protected by the $5^{\prime}$-cap and the $3^{\prime}$ - poly-A tail. Besides, plenty of reports from $E$. coli to human being revealed that the codon usage is a conserved mechanism to influence mRNA stability (Bazzini et al., 2016; Boël et al., 2016; Harigaya and Parker, 2016; Yu et al., 2015). Many codons tend to enrich in stable mRNAs, and other codons prefer to be used in unstable mRNAs, and this codon bias could enhance or reduce mRNA stability by several folds. Moreover, the previous publication has shown the decapping activator, Dhh1, tends to bind and decapping mRNA enriched with less preferred codon, thereby induces mRNA decay (Radhakrishnan et al., 2016). Furthermore, codon optimality affects translation efficiency as well, as codon optimality affects mRNA folding energy and translation 
initiation (Hanson and Coller, 2018).

As Slam is an abundant protein at the basal domain during cellularization, codon bias and different tRNA enrichment might easily be found in the slam mRNA sequence. Partial or complete replacement of the slam mRNA coding sequence with synonymous codons might introduce some less preferred codons, leading to a decrease of Slam protein elongation velocity, translation efficiency, and increase slam mRNA decay. As a result, the Slam protein expression decreased in different slam ACU constructs.

In Drosophila embryos, during cellularization, the embryo-to-embryo variation of the Slam protein amount at the furrow canal is small in wild type embryos. Whereas the variation is larger in embryos with different slam mRNA sequences (Figure 81). In wild type embryos, due to individual differences, different amounts of Slam accumulate at the basal domain can be observed in different embryos. The individual difference of Slam protein expression may be due to the change of translation efficiency and slam mRNA stability in embryos with different slam mRNA sequences, which leads to the variation of Slam protein accumulation at the basal domain.

On the other side, the localization of Slam protein at the basal domain is vital for slam mRNA localization, as it recruits slam mRNA to the basal domain. slam mRNA and the Slam protein at the basal domain form a positive feedback loop. The right localization of slam mRNA at the basal domain is essential for slam mRNA local translation. The slam mRNA local translation leads to the rapid increase of Slam protein at the basal domain. The result indicates that even for the embryos that have the same slam ACU sequence, the individual difference is very significant. The accumulation variation of Slam protein at the basal domain leads to different abilities for recruiting slam mRNA to the basal domain, this could result in a larger variation of Slam protein at the basal domain. For the quantification of Slam protein accumulation at the basal domain, only embryos that have Slam protein at the basal domain are included. The immunostaining of Slam protein indicated that in slam_acu1 and slam_acu2, slam mRNA and Slam protein are mislocalized in some embryos during cellularization. Although the variation of the Slam protein level is similar in slam_acu1 and slam_acu2, the membrane ingression is quite different between them. For slam_acu1 embryos, the membrane ingression is comparable to wild type in a good number of embryos, but for slam_acu2, the membrane ingression is affected in most embryos. This indicates membrane ingression depends on Slam protein and slam 
mRNA at the basal domain.

Furthermore, in some slam_acu1 and slam_acu2 embryos, the Slam protein is mislocalized, most of the Slam protein accumulates at the cortex of embryos. Since the amino acid sequence of Slam protein in slam_acu1 and slam_acu2 embryos is the same as the wild type, it indicates that the localization of Slam protein might also depend on the slam mRNA sequence. This result is different from the result that has been published. Yan et.al. has reported in slam ACU embryos, with the GFP-stopslam mRNA injection, the Slam protein signal did not increase, this result suggests that slam mRNA did not attract Slam protein to the furrow canal (Yan et al., 2017).

The previous report has reported that slam ACU lost the ability to accumulate at the furrow canal, but the specific region has not been identified. In this study, I found that the slam mRNA coding sequence from 507 nt to 1576 nt (Localization element 2 (Loc2) and Loc3 exist in this segment) and $2818 \mathrm{nt}$ to $3522 \mathrm{nt}$ are important for slam mRNA localization (Figure 82). On one side, in Drosophila embryos when the slam mRNA is mislocalized, the Slam protein mainly localizes to the embryo cortex instead of the basal domain during cellularization. Slam protein mislocalization leads to slam mRNA mislocalization. On the other side, the slam mRNA sequence contains information for the localization of itself. Localization information in localized RNAs is recognized by trans-acting factors, this is important for RNA transport and localization (Jambhekar et al., 2005; Meer et al., 2012). Furthermore, mRNA binding proteins have a favourite binding domain. For example, mRNA binding protein Staufen binds to Rgs4 3' UTR (Heber et al., 2019). Drosophila cytoplasmic polyadenylation element binding (CPEB) proteins tend to bind mRNA 3' UTR (Stepien et al., 2016). N-terminal of slam mRNA sequence may contain RNA binding protein target, replacing slam mRNA sequence with synonymous codons may change the affinity of slam mRNA to its RNA binding protein and induces slam mRNA decreasing at the basal domain during cellularization.

$\mathrm{I}$ identified the noncoding information in different slam mRNA regions, including the slam mRNA and protein localization and translation control in the slam mRNA sequence. Uneven distribution of slam mRNA and local translation induce the asymmetric distribution of protein, providing activity in cortical polarization. Apart from the slam mRNA sequence is essential for slam mRNA localization and protein expression, the slam mRNA sequence may also required for Slam protein localization. slam mRNA from $507 \mathrm{nt}$ to $1576 \mathrm{nt}$ affects slam mRNA localization and Slam protein 
expression. The slam mRNA sequence from $2818 \mathrm{nt}$ to $3522 \mathrm{nt}$ is required for slam mRNA localization. 


\section{Part C Materials and Methods}




\section{Materials and Methods}

\subsection{Materials}

\subsubsection{Fly stocks used in this study}

Table 8.1 Fly stocks used in this study

\begin{tabular}{|c|c|c|}
\hline Stock name & Genotype & $\begin{array}{c}\text { Source/ Lab serial } \\
\text { number }\end{array}$ \\
\hline oregon $\mathrm{R}$ & $+/+$ & A401 \\
\hline Khc RNAi & $\mathrm{y}^{1} \mathrm{sc}^{*} \mathrm{v}^{1} ; \mathrm{P}\{$ TRiP.GL00330\}attP2 & M016 \\
\hline Khc-GFP & $\mathrm{w}^{*} ;$ Khc-GFP & M031 \\
\hline GFP-slam & w; GFPslam $\{w+\}$ & $\mathrm{H} 087$ \\
\hline Moe-RFP & w; Sp/CyO; moe-RFP $\{w+\}$ & B323 \\
\hline Sqh-GFP & cv sqh[AX5]; sqh-GFP $\{w+\}$ & M001 \\
\hline His-GFP & w; Histone2Av-GFP $\{w+\}$ & B303 \\
\hline UASp-GFPslam & w; Sp/CyO; UASp-GFPSlam $\{w+\}$ & $\mathrm{H} 049$ \\
\hline Сyo;TM3 & w; Sp/CyO, hb-lacZ\{ry+\}; Dr/TM3,Sb hb-lacZ\{ry+\} & A216 \\
\hline Khc RNAi & $\mathrm{y}^{1} \mathrm{sc}^{*} \mathrm{v}^{1} ; \mathrm{P}\{$ TRiP.HMS01519\}attP2 & M017 \\
\hline TM3 TM6B & w; TM3, Sb Ser/TM6B, Tb Hu & A311 \\
\hline Ovo 2L & hs-FIp[122];ovoD Frt2L[40A]/CyO, hs-hid & Prof. J. Grosshans \\
\hline Ovo 3L & 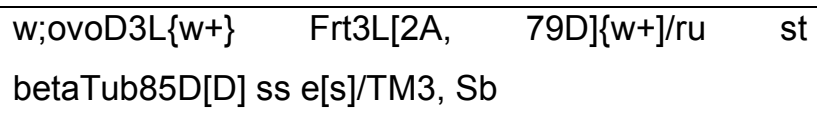 & B431 \\
\hline slam[10] & 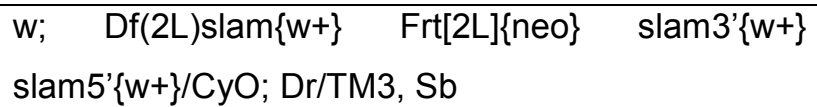 & $\mathrm{H} 039$ \\
\hline Cherry-slam & w; UASp-Cherry-slam $\{w+\}$ & $\mathrm{H} 092$ \\
\hline ELMO-GFP & w; ELMO-GFP $\{w+\}$ & $\begin{array}{l}\text { A. Schmidt; Z. Lv et al., } \\
2017\end{array}$ \\
\hline CanoeYFP & PBac $\left\{602\right.$. P. SVS-1\}cno ${ }^{\text {CPTा1000590 }}$ & Kyoto Stock Center \\
\hline EB1-GFP(II) & w; UASp-EB1-GFP $\{w+\} / C y O$ & B333 \\
\hline Khc-mKate & Khc[mKate2]/CyO & Dr. Anne Ephrussi \\
\hline UASp-GFPutr & w; UASp-GFP-UtrophinABD $\{w+\} / C y O$ & B325 \\
\hline APC2 d40 & Apc2 d40 ca ${ }^{1} / \mathrm{TM} 3, \mathrm{Sb}^{1}$ & Dr. B. M. McCartney \\
\hline APC2 g10 & Apc2 g10 $\mathrm{ca}^{1} / \mathrm{TM} 3, \mathrm{Sb}^{1}$ & Dr. B. M. McCartney \\
\hline APC2-GFP & $w^{*} ; P\{w[+m C]=U A S-A p c 2 . G F P\} 3 / T M 3, S b^{1}$ & $\begin{array}{l}\text { Bloomington Stock } \\
\text { Center }\end{array}$ \\
\hline Cpa-GFP & Cpa-GFP & Prof. J. Grosshans \\
\hline MTD-Gal4 & $\begin{array}{l}\text { P[otu-Gal4::VP16.R]1,w*;P[Gal4-nos.NGT]40;P[G } \\
\text { al4::VP16nos.UTR]CG6325(MVD1) }\end{array}$ & $\begin{array}{l}\text { Bloomington Stock } \\
\text { Center }\end{array}$ \\
\hline
\end{tabular}


8.1.2 Fly stocks generated in this study

Table 8.2 Fly stocks generated in this study

\begin{tabular}{|c|c|}
\hline Name & Description \\
\hline slam_acu1 & w; Df(2L)slam $\{w+\}$ Frt[2L] $\{$ neo $\}$ slam3' $\{w+\}$ slam5' $\{w+\} / C y O ;$ slamacu1 $\{w+\}$ \\
\hline slam_acu2 & w; Df(2L)slam $\{w+\}$ Frt[2L] $\{$ neo $\}$ slam3' $\{w+\}$ slam5' $\{w+\} / C y O ;$ slamacu2 $\{w+\}$ \\
\hline slam_acu3 & w; Df(2L)slam $\{w+\}$ Frt[2L]\{neo $\}$ slam3' $\{w+\}$ slam5' $\{w+\} / C y O ;$ slamacu3 $\{w+\}$ \\
\hline
\end{tabular}

\subsubsection{Oligonucleotides}

All oligonucleotides used in this study were ordered from Eurofins genomics.

Table 8.3 Oligonucleotides used in this study

\begin{tabular}{|c|c|c|}
\hline Oligo NO. & Sequence (from 5' to 3') & Description \\
\hline LL4 & GAACTAGTGGATCGCCCTG & $\begin{array}{l}\text { senser for } \quad \text { slam_acu1 } \\
\text { from } 681 \mathrm{bp}\end{array}$ \\
\hline LL5 & CGAGATCTCCGGACGCAAACCAGTAATGG & $\begin{array}{l}\text { antisenser for slam_acu3 } \\
\text { from } 4122 \text { bp with Bgl II }\end{array}$ \\
\hline LL6 & TTCGCTCCCCAAATCAAAGTTC & $\begin{array}{l}\text { antisenser for slam_acu1 } \\
\text { from } 3053 \text { bp }\end{array}$ \\
\hline LL7 & GAGATCTGGCCGTCCCGTTTTCCGG & $\begin{array}{l}\text { senser for slam_acu3 } \\
\text { from } 4122 \text { bp with Bgl II }\end{array}$ \\
\hline LL9 & TCGCGCTAACCGATATTTA & $\begin{array}{l}\text { antisenser for slam_acu2 } \\
\text { from } 1087 \mathrm{bp}\end{array}$ \\
\hline SY77 & GATGAATAGCGACGACCTGTC & slam sequence primer \\
\hline SY78 & CAAAGTGTCGGCCTCCCTC & slam sequence primer \\
\hline SY79 & ACGACGCTGTCTCGACCGCC & slam sequence primer \\
\hline SY80 & GCTTGAGCCAGGTGACGAG & slam sequence primer \\
\hline SY81 & CGCTGACGGTCATGAGCTC & slam sequence primer \\
\hline SY82 & GCGATTTGGCGTTTGGTATTG & slam sequence primer \\
\hline SY83 & CCGAATTTGAAAGCGACTCC & slam sequence primer \\
\hline SY84 & CGGACAGGTCGTCGCTATTC & slam sequence primer \\
\hline JG107 & AATTAACCCTCACTAAAGGG & T7 primer \\
\hline ZL96 & GTAATACGACTCACTATAGGGC & T3 primer \\
\hline
\end{tabular}


8.1.4 Primary antibodies

Table 8.4 Primary antibodies used in this study

\begin{tabular}{|c|c|c|c|c|}
\hline \multirow[t]{2}{*}{ Antibody } & \multirow[t]{2}{*}{ Raised in } & \multicolumn{2}{|c|}{ Dilution } & \multirow[t]{2}{*}{ Source } \\
\hline & & staining & Western & \\
\hline Slam & Rabbit & $1: 5000$ & - & Jörg Großhans \\
\hline Slam & Guinea Pig & $1: 5000$ & $1: 5000$ & Jörg Großhans \\
\hline Nullo & Mouse & $1: 10$ & - & Hybridoma bank 5C3-12 \\
\hline Nuf & Rabbit & $1: 1000$ & - & Willian Sullivan \\
\hline Y-Tubulin & Mouse & $1: 5000$ & - & Sigma T6557 \\
\hline Lamin Dmo & Mouse & $1: 1000$ & $1: 1000$ & Würzburg Krohne \\
\hline Canoe & Rabbit & $1: 1000$ & - & Mark Peifer \\
\hline Sponge & Guinea Pig & $1: 1000$ & - & Erika R. Geisbrecht \\
\hline$\alpha-T u b u l i n$ & Mouse & $1: 5000$ & $1: 50000$ & Sigma Aldrich (St.Louis, USA) \\
\hline Dlg & Mouse & $1: 100$ & $1: 500$ & Hybridoma Bank 4F3 \\
\hline GFP & Rabbit & $1: 500$ & $1: 10000$ & $\begin{array}{l}\text { Torrey Pines Biolabs } \\
\text { (Seacaucus, USA) }\end{array}$ \\
\hline Capping $\alpha$ & Rabbit & $1: 200$ & $1: 500$ & Florence Janody \\
\hline Capping $\beta$ & Rabbit & $1: 200$ & & Florence Janody \\
\hline Zipper & Rabbit & $1: 200$ & $1: 50000$ & Jeffrey Thomas \\
\hline Dia & Rabbit & $1: 1000$ & $1: 1000$ & Jörg Großhans \\
\hline APC2 & Rabbit & $1: 500$ & $1: 1000$ & Julius Mieszczanek \\
\hline Amphiphysin & Guinea Pig & $1: 1000$ & - & Jörg Großhans \\
\hline Bazooka & Rabbit & $1: 1000$ & $1: 2000$ & Andreas Wodarz \\
\hline Armadillo & Mouse & $1: 50$ & - & Hybridoma Bank N2 7A1 \\
\hline
\end{tabular}

Secondary antibodies conjugated with Alexa were used in a final concentration of 4 $\mu \mathrm{g} / \mu \mathrm{l}$ (1:500). Alexa-conjugated Phalloidin used for F-actin staining was used with dilution of 1:1000. Both products were purchased from Invitrogen (Carlsbad, USA). GFP-booster-Atto488 was used at a final concentration of $2 \mu \mathrm{g} / \mathrm{ml}$ (1:500) and purchased from Chromotek (Martinsried, Germany). Anti-Digoxigenin-peroxidase antibodies were purchased from Roche (Basel, Switzerland) and used at a final dilution of 1:200. Secondary antibodies for western blots IRDye-800CW and IRDye680 were used at a final concentration of $0.05 \mu \mathrm{g} / \mathrm{ml}(1: 20000)$ and purchased from LI-COR Biotechnology (Bad Homburg, Germany).

8.1.5 Buffers used in this study

a) DNA extraction buffers (Buffer $A$ and Buffer B) 


\section{Buffer A}

30 mM Tris/HCl [pH 8]; 100 mM NaCl; 19 mM EDTA; 0.5\% Triton X-100

Buffer B

30 mM Tris/HCl [pH 8]; 100 mM NaCl; 19 mM EDTA

b) Phosphate buffered saline (PBS [pH 7.4])

130 mM NaCl; 7 mM Na2HPO4; 3 mM NaH2PO4

c) PBT

PBS with $0.1 \%$ Tween 20

d) Immunoprecipitation buffer (IP buffer)

$50 \mathrm{nM}$ Hepes/NaOH, [pH 7.5]; 150 mM NaCl; 0.5\% Triton X-100; 10\% Glycerin; 1 mM PMSF; 1 mM EGTA; 1 mM EDTA; ROCHE

e) 6x Lämmli buffer

375 mM Tris/HCl; 10\% SDS; 50\% Glycerol; 0.6 M DTT; 0.06\% Bromophenole blue

f) Western blot buffers (Running buffer and Transfer buffer)

Running buffer

0.25 M Tris; 2 M Glycine; 1\% SDS

Transfer buffer

25 mM Tris; 175 mM Glycine; 20\% Methanol

g) TAE buffer

10 mM Tris/HCl [ $\mathrm{pH} 8.0$ ]; 1 mM EDTA

h) Drosophila embryos heat fixation buffer

$0.4 \% \mathrm{NaCl} ; 0.03 \%$ Triton $\mathrm{X}-100$

i) Drosophila embryos formaldehyde fixation buffer (in total $5 \mathrm{ml}$ )

$4.5 \mathrm{ml}$ 1x PBS; $0.5 \mathrm{ml}$ or $1 \mathrm{ml}$ Formaldehyde (37\%); $0.5 \mathrm{ml}$ Heptane

j) Hybridization solution (stored at $-20^{\circ} \mathrm{C}$ ) 
$50 \%$ formamide; $5 x$ SSC; $50 \mu \mathrm{g} / \mathrm{ml}$ heparin; 0.2\% Tween; $100 \mu \mathrm{g} / \mathrm{ml}$ tRNA; water

\subsubsection{Kits}

MiniElute Gel extraction Kit Quiagen, Hilden

Plasmid Midi Kit Nucleobond AX Macherey-Nagel, Düren

\subsubsection{Bacterial cell line}

DH5a for molecular cloning: F- Ф80lacZ $\Delta \mathrm{M} 15 \Delta($ lacZYA-argF) U169 recA1 endA1 hsdR17 (rK-, mK+) phoA supE44 $\lambda-$ thi-1 gyrA96 relA1

\subsubsection{Plasmids}

Table 8.5 Plasmids used in this study

\begin{tabular}{|l|l|l|}
\hline \multicolumn{1}{|c|}{ Name } & \multicolumn{1}{|c|}{ Description } & \multicolumn{1}{|c|}{ Source } \\
\hline slam attB 8.5 & $\begin{array}{l}\text { Insert slam sequence, generate transgenic flies } \\
\text { with attB/phi-C31 system }\end{array}$ & Jörg Großhans \\
\hline BKSIl-slam_fl_acu & $\begin{array}{l}\text { slam full length codon degeneracy expresssion } \\
\text { in E.coli }\end{array}$ & Jörg Großhans \\
\hline BKSII-BamH_acu & $\begin{array}{l}\text { slam (507 nt to 1576 nt) codon degeneracy } \\
\text { expresssion in E.coli }\end{array}$ & Jörg Großhans \\
\hline BKSII-slam & slam full length expresssion in E.coli & Jörg Großhans \\
\hline
\end{tabular}

Table 8.6 Plasmids generated in this study

\begin{tabular}{|l|l|}
\hline \multicolumn{1}{|c|}{ Name } & \multicolumn{1}{c|}{ Description } \\
\hline BKS-slam_acu1 & slam (1 nt to 1576 nt) codon degeneracy expresssion in E.coli \\
\hline BKS-slam_acu2 & slam (685 nt to 3522 nt) codon degeneracy expresssion in E.coli \\
\hline BKS-slam_acu3 & slam (685 nt to 2818 nt) codon degeneracy expresssion in E.coli \\
\hline
\end{tabular}

\subsubsection{Microscopes}

Confocal microscopy LSM780 equipped with AiryScan (Carl Zeiss), Zeiss Axiovert 200M Ultra-view spinning Disc confocal microscope (Carl Zeiss), Microinjection microscope (Carl Zeiss), Stereomicroscopes (Carl Zeiss). 


\subsubsection{Other materials}

Table 8.7 Other materials used in this study

\begin{tabular}{|l|l|}
\hline \multicolumn{1}{|c|}{ Products } & \multicolumn{1}{c|}{ Source } \\
\hline GFP-sepharose beads & ChromoTek, Germany \\
\hline Glass slides & Thermo Scientific \\
\hline Fly vials & Greiner \\
\hline Glass pipettes $(25 \mathrm{ml}, 20 \mathrm{ml}, 10 \mathrm{ml}, 5 \mathrm{ml})$ & Brandt \\
\hline Petri dishes & Greiner \\
\hline Pipet-aid & Drummond \\
\hline Micropipettes $(1000 \mu \mathrm{l}, 200 \mu \mathrm{l}, 20 \mu \mathrm{l}, 2 \mu \mathrm{l})$ & Gilson \\
\hline Micropipette tips $(1000 \mu \mathrm{l}, 200 \mu \mathrm{l}, 20 \mu \mathrm{l}, 2 \mu \mathrm{l})$ & Eppendorf \\
\hline Eppendorf tubes $(1.5 \mathrm{ml}, 2 \mathrm{ml}, 5 \mathrm{ml})$ & Eppendorf \\
\hline PCR tubes & Brand \\
\hline Falcon tubes $(50 \mathrm{ml}, 15 \mathrm{ml})$ & BD Falcon \\
\hline
\end{tabular}

\subsubsection{Other equipments}

Table 8.8 Other equipments used in this study

\begin{tabular}{|l|l|}
\hline \multicolumn{1}{|c|}{ Products } & \multicolumn{1}{c|}{ Source } \\
\hline Centrifugal & Eppendorf \\
\hline Odyssey CLx Infrared imaging system & LI-COR Biosciences \\
\hline Thermal Cycler & Bio-rad \\
\hline Microinjector & FemtoJet - Eppendorf \\
\hline
\end{tabular}

\subsubsection{Softwares}

Table 8.9 Softwares used in this study

\begin{tabular}{|l|l|}
\hline \multicolumn{1}{|c|}{ Softwares } & \\
\hline Office (Word, Excel, Powerpoint) & Microsoft \\
\hline Adobe Photoshop & Adobe \\
\hline Adobe illustrate & Adobe \\
\hline Image J & NIH, USA \\
\hline Zen 2012 & Carl Zeiss \\
\hline Lasergene & GATC biotech \\
\hline
\end{tabular}




\subsection{Methods}

\subsubsection{Fixation of Drosophila embryos (Heat fixation and Formaldehyde fixation)}

\section{a) Heat fixation}

Drosophila embryos at the right stage were dechorionated with $50 \%$ Klorix for $1.5 \mathrm{~min}$ and then embryos were collected with a net. Embryos in the net were washed with water to remove Klorix. Then collected embryos were put into boiled heat fixation buffer and incubated for $10 \mathrm{~s}$. After $10 \mathrm{~s}$, the buffer was cooled down with ice. The heat fixation buffer was removed after embryos sunk to the bottom, $5 \mathrm{ml}$ heptane and $5 \mathrm{ml}$ methanol were added into the sample. Spun embryos for $20 \mathrm{~min}$. Embryos were then collected into Eppendorf tube and washed with methanol for 3 times, then embryos were stored at $-20^{\circ} \mathrm{C}$ with methanol.

\section{b) Formaldehyde fixation}

Drosophila embryos at the right stage were dechorionated with $50 \%$ Klorix for $1.5 \mathrm{~min}$ and then embryos were collected with a net, embryos in the net were washed with water to remove Klorix. After washing, embryos were transferred into the bottle with $0.5 \mathrm{ml}$ formaldehyde, $4.5 \mathrm{ml}$ PBS and $5 \mathrm{ml}$ heptane. Spun embryos for $20 \mathrm{~min}$. Following this, PBS and formaldehyde were removed and $5 \mathrm{ml}$ methanol was added to the bottle, shaken violently for $30 \mathrm{~s}$. Embryos were then collected into Eppendorf tube and washed with methanol for 3 times, then embryos were stored at $-20^{\circ} \mathrm{C}$ with methanol.

\subsubsection{DNA extraction from Drosophila adults}

About 10 adult flies were collected into $1.5 \mathrm{ml}$ Eppendorf tube and fixed with liquid nitrogen. Then $90 \mu$ buffer $A$ was added into the tube and grinded with pestle grinder for $1 \mathrm{~min}$, spun for $2 \mathrm{~min}$. After centrifugation, the supernatant was removed, washed sample with $200 \mu \mathrm{l}$ buffer A, spun again and the supernatant was discarded. $90 \mu \mathrm{l}$ buffer $B, 0.9 \mu \mathrm{l}$ protease $\mathrm{K}$, and $10 \mu \mathrm{l} 10 \%$ SDS were added. The sample was mixed and incubated for $1 \mathrm{~h}$ at $37^{\circ} \mathrm{C}$. After incubation, $15 \mu \mathrm{N} \mathrm{NaCl}(3 \mathrm{M}), 50 \mu \mathrm{l}$ phenol/chloroform were added and mixed. Following this step, spun sample for 2 min and transferred $100 \mu \mathrm{l}$ supernatant to a new tube. The supernatant was mixed with $250 \mu \mathrm{l}$ ethanol, spun, and removed supernatant. Pellet was washed with $70 \%$ ethanol, 
dissolved with water after pellet dry.

\subsubsection{Cloning of different hybrid slam mRNA sequences}

a) To get BKSII (+)-slam_acu1, plasmids BKSII (+)-slam_fl_acu and BKSII (+)-slam were used. BKSII (+)-slam_fl_acu and BKSII (+)-slam were cut with restriction enzymes Hpal and BamHI. Different fragments after digestion were separated and obtained with agarose gel electrophoresis. The $597 \mathrm{bp}$ and $5649 \mathrm{bp}$ fragments were obtained from BKSII (+)-slam, the 1069 bp fragment was obtained from BKSII (+)slam_fl_acu. Ligating 597 bp and 5649 bp fragments together with T4 ligase. After ligation, the 6246 bp plasmid was transformed and amplified in $\mathrm{DH} 5 \alpha$ bacterial cell line. The amplified plasmid was cleaved with BamHI (vector) and the $1069 \mathrm{bp}$ fragment from BKSII (+)-slam_fl_acu was inserted into the vector. The BKSII (+)slam_acu1 was obtained with ligation.

b) To get BKSII (+)-slam_acu2, BKSII (+)-slam_fl_acu and BKSII (+)-BamH_acu were digested with restriction enzymes BstBI and Smal. 1510 bp, 1621 bp, and 4184 bp fragments were isolated from BKSII (+)-slam_fl_acu, 3131 bp and 4184 bp fragments were obtained from BKSII (+)-BamH_acu. Ligating the 1510 bp fragment from BKSII (+)-slam_fl_acu and the 4184 bp fragment from BKSII (+)-BamH_acu together. After ligation, the plasmid was transformed and amplified in $\mathrm{DH} 5 \mathrm{a}$. The amplified plasmid (vector) was cut with BstBI, the 1621 bp fragment from BKSII (+)slam_fl_acu was inserted into the vector, the BKSII (+)-slam_acu2 was obtained.

c) To get BKSII (+)-slam_acu3, primers LL5 (contains Bglll recognizing site) and T7 were used. The plasmid BKSII (+)-BamH_acu was used as the template. The PCR product (3497bp) and BKSII (+)-slam_acu2 were digested with restriction enzymes $\mathrm{Bgl} \mathrm{II}$ and Hpal. After agarose gel electrophoresis, the $3877 \mathrm{bp}$ fragment (vector) was obtained from BKSII (+)-slam_acu2 and the 3438 bp fragment was obtained from the PCR product. 3877 bp and 3438 bp fragments were ligated together with T4 ligase, and the BKSII (+)-slam_acu2 was obtained.

Different BKSII (+)-slam_acu constructs and attB-slam 8,5 were cut with Pasl and Sphl. After agarose gel electrophoresis, three $4346 \mathrm{bp}$ fragments were isolated from different BKSII (+)-slam_acu constructs, the 11713 bp fragment (vector) was isolated from attB-slam 8,5. The three 4346 bp fragments were inserted into vector respectively with T4 ligase, plasmids were transformed and amplified. 
8.2.4 Plasmid DNA purification and amplification

All cloning works followed the description in Sambrook and Russel, 2001.

8.2.5 Polymerase chain reaction (PCR)

a) Reagents used in PCR $(20 \mu \mathrm{l})$ were mixed as follow:

Table 8.10 Reagents used in PCR

\begin{tabular}{|l|l|}
\hline \multicolumn{1}{|c|}{ Reagents } & \multicolumn{1}{c|}{ Usage } \\
\hline PCR buffer & $5 x$ phusion HF buffer $4 \mu \mathrm{l}$ or $10 x$ Taq buffer $2 \mu \mathrm{l}$ \\
\hline dNTP & $0.4 \mu \mathrm{l}$ \\
\hline Primers & $1 \mu \mathrm{l}$ sense and antisense \\
\hline Template & $200 \mathrm{ng}$ DNA template \\
\hline Polymerase & $0.2 \mu \mathrm{l}$ \\
\hline Water & add to $20 \mu \mathrm{l}$ \\
\hline
\end{tabular}

Polymerases (Taq and Pfus) used in PCR generated in the lab.

b) PCR program in this study:

Table 8.11 PCR program

\begin{tabular}{|l|l|l|}
\hline \multicolumn{1}{|c|}{ Step } & \multicolumn{1}{|c|}{ Temperature } & \multicolumn{1}{c|}{ Duration } \\
\hline 1. Initial denaturation & $98.5^{\circ} \mathrm{C}$ & $3 \mathrm{~min}$ \\
\hline 2. Denaturation & $98.5^{\circ} \mathrm{C}$ & $30 \mathrm{~s}$ \\
\hline 3. Annealing & $62^{\circ} \mathrm{C}$ & $30 \mathrm{~s}$ \\
\hline 4. Extension & $72^{\circ} \mathrm{C}$ & $1 \mathrm{~kb} / \mathrm{min}$ \\
\hline 5. Final extension & $72^{\circ} \mathrm{C}$ & $10 \mathrm{~min}$ \\
\hline
\end{tabular}

Step 2 to 4 were repeated for 30 times.

\subsubsection{In-fusion cloning}

Restriction enzymes and T4 ligase used for DNA ligation in this study were followed with instructions of products.

\subsubsection{DNA sequencing}

DNA samples were sequenced by Microsynth Seqlab $\mathrm{GmbH}$. 


\subsection{Protein methods}

\subsubsection{Western blot}

Embryos at the right stage were dechorionated with $50 \%$ Klorix for $1.5 \mathrm{~min}$. Embryos were collected with a net and then washed with water to remove Klorix. Following washing, embryos were transferred into Eppendorf tube (about 40 embryos), quickly fixed with liquid nitrogen. Then the sample was homogenized with $20 \mu \mathrm{l} 1 \mathrm{x}$ Lämmli buffer, the sample was incubated at $100^{\circ} \mathrm{C}$ for $5 \mathrm{~min}$. After incubation, the sample was centrifuged for 10 min with $14000 \mathrm{rpm}$, the supernatant was obtained.

The supernatant was loaded in SDS-PAGE gel well. After electrophoresis, proteins were transferred onto the nitrocellulose membrane with the semi-dry method. Then the nitrocellulose membrane was blocked with 5\% BSA for 1 hour and then incubated with first antibody for 2 hours at room temperature. After incubation, the nitrocellulose membrane was rinsed for 3 times with PBT, the nitrocellulose membrane was washed with PBT for 3 times and $10 \mathrm{~min}$ for each time. Then the nitrocellulose membrane was incubated with secondary antibody for 2 hours at room temperature. After incubation, the nitrocellulose membrane was rinsed for 3 times with PBT, the nitrocellulose membrane was washed with PBT for 3 times and 10 min for each time. The result was obtained with the Odyssey CLx Infrared Imaging System.

\subsubsection{Immunoprecipitation}

Kinesin1-GFP and wild type embryos at the right stage were dechorionated with $50 \%$ Klorix for 1.5 min, embryos were collected with nets respectively. Following this, embryos were washed with water to remove Klorix. Collected embryos were transferred into Eppendorf tubes and weighed (1 mg=100 embryos). Then embryos were quickly fixed in liquid nitrogen. $100 \mathrm{mg}$ embryos were prepared for each sample, samples were homogenized in Dounce homogenizers with $1 \mathrm{ml} I \mathrm{P}$ buffer. Samples were lysed and transferred into $1.5 \mathrm{ml}$ Eppendorf tubes. Following this, samples were spun for $15 \mathrm{~min}$ with $14000 \mathrm{rpm}$ at $4^{\circ} \mathrm{C}$. At the same time, preparing GFP-sepharose beads for each sample, $20 \mu$ l GFP-sepharose beads for each sample were washed with PBS and spun 2000 rpm for 2 min. After centrifugation, lipid above supernatant was removed. $1 \mu \mathrm{l}$ supernatant for each sample was collected as the input group, mixed with 1x Lämmli buffer. Then supernatant of each sample was incubated with 
prepared GFP-Sepharose beads for 1 hour at $4^{\circ} \mathrm{C}$. After incubation, samples were spun for 1 min with $2000 \mathrm{rpm}$, supernatant of each sample was collected as the unbound group, mixed with 1x Lämmli buffer. GFP-sepharose beads were washed for 4 times with PBS and eluted with $50 \mu \mathrm{l}$ 1x Lämmli buffer, supernatant for each sample was collected as the bound group. All groups including input, unbound and bound were incubated in $100^{\circ} \mathrm{C}$ for $5 \mathrm{~min}$, centrifuged for $10 \mathrm{~min}$ with $14000 \mathrm{rpm}$. After centrifugation, western blot was used to check protein-protein interaction.

\subsubsection{Immunostaining}

The immunostaining procedures were followed with the description in the publication (Wenzl et al., 2010).

\subsubsection{Rho kinase inhibitor Y-27632 injection and immunostaining}

Y-27632 was injected into pre-blastoderms expressing Cpa-GFP. Embryos at the right stage were dechorionated, lining up onto coverslips. Y-27632 was dissolved into water to $10 \mathrm{mM}$ and injected into embryos at the posterior side, embryos were incubated in room temperature for $20 \mathrm{~min}$. After incubation, embryos were fixed with $8 \%$ formaldehyde for $20 \mathrm{~min}$. Following with embryos fixation, embryos were peeled and stained with F-actin, Myosin II and Cpa-GFP.

\subsubsection{In situ hybridization}

Embryos at the right stage were dechorionated with $50 \%$ Klorix for $1.5 \mathrm{~min}$. Embryos were collected with a net and washed with water to remove Klorix. Embryos were fixed with $8 \%$ formaldehyde, then embryos were transfered to a new Eppendorf tube. Embryos were then rinsed 3 times and washed 2 times with PBT. Following this, embryos were incubated with hybridization solution (hyb-sol)/PBT (1:1) for $10 \mathrm{~min}$, then embryos were incubated with hyb-sol for $10 \mathrm{~min}$. The slam RNA probe was prehybridised for 1 hour at $57^{\circ} \mathrm{C}$. After incubation with hyb-sol, samples were mixed with the slam RNA probe in hyb-sol and incubated at $57^{\circ} \mathrm{C}$ overnight. Following this, embryos were rinsed 3 times with pre-warmed hyb-sol, washed with pre-warmed hyb-sol for $30 \mathrm{~min}$ at $57^{\circ} \mathrm{C}$, repeated washing 2 times. Then embryos were incubated with different ratios of hyb-sol/PBT (4:1, 3:2 and 2:3 respectively) for 10 min at $57^{\circ} \mathrm{C}$. After these steps, embryos were washed with hyb-sol/PBT (1:4) in room temperature 
for 10 min. After washing, embryos were incubated in PBT with 1\% BSA for 20 min at room temperature, repeated once, rinsed with PBT. Digoxigenin antibody couples with peroxidate at dilution 1:200 with PBT were added to embryos, incubated at room temperature for 2 hours. Embryos were then rinsed with PBT 3 times and washed with PBT for $15 \mathrm{~min}$, repeated 3 times. Embryos were stained with $200 \mu$ l solution buffer (TSA-Cy3 stock/reaction buffer = 1:200) without light for $10 \mathrm{~min}$, then embryos were washed with PBT to stop staining. Following with immunostaining and DAPI staining.

DEPC treatment: add $2 \mathrm{ml}$ diethyl pyrocarbonate per $1 \mathrm{I}$ of water/solution, incubate at $37^{\circ} \mathrm{C}$ overnight and autoclave.

All buffers and reagents in this experiment were RNAase free or treated with DEPC. slam RNA probe was supplied by Dr. Shuling Yan.

\subsection{Transgenic flies}

\subsubsection{Cpa-GFP fly}

The Cpa-GFP fly was generated by the inDroso functional genomics company. eGFP sequence and stop codon were inserted behind the Cpa (CG10540) coding sequence by CRISPR. The guide RNA is ACTGAAGACGCAATAAGACC AGG. Below was the structure of the donor plasmid, the linker is GVG.

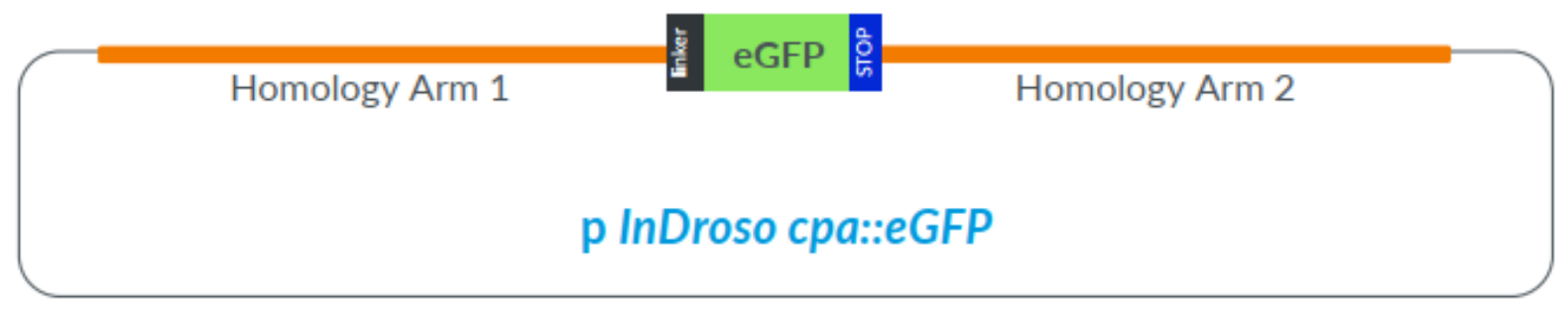

\subsubsection{Generation of transgenic flies}

The transgenic flies were generated by using attB/phi-C31-based integration system. The process for generating transgenic flies was followed with a standard protocol (Wenzl et al., 2010) (http://wwwuser.gwdg.de/ jgrossh/method). 


\subsection{Imaging}

\subsubsection{Imaging for fixed embryos}

Fixed and immunostained embryos were obtained by Confocal microscopy LSM780 with $63 x$ oil objective. Surface and section views of embryos were obtained with $2 x$ zoom, the size of images was $67.5 \times 67.5 \mu \mathrm{m}(512 \times 512$ pixel), the pixel size of images was $130 \mathrm{~nm}$. Z-stack size of each step was $0.5 \mu \mathrm{m}$.

\subsubsection{Live imaging}

Differential interference contrast (DIC) microscopy by Ultra-view spinning Disc confocal microscope with $40 x$ oil objective was used to observe the development of Drosophila embryos.

For live imaging, embryos were handled as described before (Kanesaki et al., 2011). For live imaging of embryos expressing either Khc-GFP and Slam-mCherry or the embryos expressing Canoe-YFP was obtained by Confocal microscopy LSM780 with $63 x$ oil objective. Surface views of embryos were obtained with $2 x$ zoom, the size of images was $67.5 \times 67.5 \mu \mathrm{m}$ ( $512 \times 512$ pixel), the pixel size of images was $130 \mathrm{~nm}$. Z-stack size of each step was $0.5 \mu \mathrm{m}$, the time interval was $1 \mathrm{~min}$. The rest of live imagings were conducted with Airyscan. Surface views of embryos were obtained with $2 x$ zoom, the size of images was $67.5 \times 67.5 \mu \mathrm{m}$ (512 x 512 pixel), the pixel size of images was $130 \mathrm{~nm}$. Z-stack size of each step was $0.5 \mu \mathrm{m}$, the time interval in different experiments were explained in figure legends.

\subsubsection{Fluorescence recovery after photobleaching (FRAP) experiment}

The FRAP experiment was conducted with Confocal microscopy LSM780. For photobleaching of GFP-Slam, the laser intensity was set to $50 \%$ and bleaching was done within fast FRAP mode. Imaging started $10 \mathrm{~s}$ before photobleaching, the time interval of live imaging was $10 \mathrm{~s}$. The size of images was $67.5 \times 67.5 \mu \mathrm{m}(512 \times 512$ pixel), the pixel size of images was $130 \mathrm{~nm}$. Three stacks were recorded, z-stack size of each step was $0.5 \mu \mathrm{m}$, z-stacks were merged for quantification. 


\subsubsection{Quantifications}

All measurements for quantification were done with Fiji/lmageJ and further calculations were finished in GraphPad prism 6.

(a) Quantification of nuclear elongation and membrane invagination

For the length of nuclei and length of furrow canal during cellualrization, measurements were done by measuring lengths from surface of embryos to front tips of nuclei or furrow canal at the indicated time points. Mean values of the length of nuclei and length of furrow canal were obtained from 3 embryos per genotype, 5 measurements in each embryo at different time point were conducted. For different furrow lengths of slam_acu , different numbers of embryos were measured in 40 min after the membrane invagination started.

(b) Measurements of $\mathrm{Cpa}$ fluorescent intensities from the edge of cap and fluorescent intensities of different protein at the cap domain (F-actin and Cpa) The quantification of Cpa fluorescent intensities in wild type, Kinesin-1 RNAi, and embryos wiht ROCK inhibitor injection was done by drawing a line perpendicular to the edge across the edge, the position where the peak of fluorescent intensity located was defined as $0 \mu \mathrm{m}$. Peaks of fluorescent intensities were normalized to 1 . The measurements for every embryo was averaged and plotted with SEM against the position. For quantifications of F-actin and $\mathrm{Cpa}$ at the cap domain, total amounts of F-actin and Cpa fluorescent intensities at the cap domain in wild type embryos were measured and normalized to 1. The F-actin and Cpa fluorescent intensities in Kinesin-1 RNAi and embryos with ROCK inhibitor injection were normalized according to wild type.

(c) Cpa-GFP clusters index $\left(\rho \% \rho^{i}\right)$

Cpa-GFP clusters at the cap domain and intercap domain were counted in every genotype at the interphase ( $0 \mathrm{~min}$ and $2 \mathrm{~min}$ later), also the area of the counting place was also measured. $\rho=$ (the number of Cpa-GFP clusters)/(the area). The CpaGFP clusters index between intercap and cap domains at different time points in different genotypes were calculated according to the density of Cpa-GFP clusters at the cap domain $\left(\rho^{i}\right)$ and intercap domain $\left(\rho^{\circ}\right)$. 


\section{References}

Aakalu, G., Smith, W.B., Nguyen, N., Jiang, C., Schuman, E.M., 2001. Dynamic visualization of local protein synthesis in hippocampal neurons. Neuron 30, 489-502.

Acharya, S., Laupsien, P., Wenzl, C., Yan, S., Großhans, J., 2014. Function and dynamics of slam in furrow formation in early Drosophila embryo. Developmental Biology 386, 371-384. https://doi.org/10.1016/j.ydbio.2013.12.022

Afshar, K., Stuart, B., Wasserman, S.A., 2000. Functional analysis of the Drosophila diaphanous FH protein in early embryonic development. Development 127, 1887-1897.

Aizawa, H., Sekine, Y., Takemura, R., Zhang, Z., Nangaku, M., Hirokawa, N., 1992. Kinesin family in murine central nervous system. J. Cell Biol. 119, 1287-1296. https://doi.org/10.1083/jcb.119.5.1287

Amândio, A.R., Gaspar, P., Whited, J.L., Janody, F., 2014. Subunits of the Drosophila actin-capping protein heterodimer regulate each other at multiple levels. PLoS ONE 9, e96326. https://doi.org/10.1371/journal.pone.0096326

Baas, P.W., Rao, A.N., Matamoros, A.J., Leo, L., 2016. Stability properties of neuronal microtubules. Cytoskeleton (Hoboken) 73, 442-460. https://doi.org/10.1002/cm.21286

Babkoff, A., Cohen-Kfir, E., Aharon, H., Ronen, D., Rosenberg, M., Wiener, R., Ravid, S., 2019. A direct interaction between survivin and myosin $\|$ is required for cytokinesis. J. Cell. Sci. 132. https://doi.org/10.1242/jcs.233130

Basnet, N., Nedozralova, H., Crevenna, A.H., Bodakuntla, S., Schlichthaerle, T., Taschner, M., Cardone, G., Janke, C., Jungmann, R., Magiera, M.M., Biertümpfel, C., Mizuno, N., 2018. Direct induction of microtubule branching by microtubule nucleation factor SSNA1. Nat Cell Biol 20, 1172-1180. https://doi.org/10.1038/s41556-018-0199-8

Bazzini, A.A., Del Viso, F., Moreno-Mateos, M.A., Johnstone, T.G., Vejnar, C.E., Qin, Y., Yao, J., Khokha, M.K., Giraldez, A.J., 2016. Codon identity regulates mRNA stability and translation efficiency during the maternal-to-zygotic transition. EMBO J. 35, 2087-2103. https://doi.org/10.15252/embj.201694699

Bement, W.M., Forscher, P., Mooseker, M.S., 1993. A novel cytoskeletal structure involved in purse string wound closure and cell polarity maintenance. The Journal of Cell Biology 121, 565-578. https://doi.org/10.1083/jcb.121.3.565

Benink, H.A., Bement, W.M., 2005. Concentric zones of active RhoA and Cdc42 around single cell wounds. The Journal of Cell Biology 168, 429-439. https://doi.org/10.1083/jcb.200411109

Bi, J., Tsai, N.-P., Lu, H.-Y., Loh, H.H., Wei, L.-N., 2007. Copb1-facilitated axonal transport and translation of kappa opioid-receptor mRNA. Proc. Natl. Acad. Sci. U.S.A. 104, 13810-13815. https://doi.org/10.1073/pnas.0703805104

Bienkowska, D., Cowan, C.R., 2012. Centrosomes can initiate a polarity axis from any position within onecell C. elegans embryos. Curr. Biol. 22, 583-589. https://doi.org/10.1016/j.cub.2012.01.064

Bilder, D., Li, M., Perrimon, N., 2000. Cooperative Regulation of Cell Polarity and Growth by Drosophila Tumor Suppressors. Science 289, 113-116. https://doi.org/10.1126/science.289.5476.113

Bilder, D., Perrimon, N., 2000. Localization of apical epithelial determinants by the basolateral PDZ protein Scribble. Nature 403, 676-680. https://doi.org/10.1038/35001108

Bilder, David, Perrimon, N., 2000. Localization of apical epithelial determinants by the basolateral PDZ protein Scribble. Nature 403, 676. https://doi.org/10.1038/35001108

Boël, G., Letso, R., Neely, H., Price, W.N., Wong, K.-H., Su, M., Luff, J.D., Valecha, M., Everett, J.K., Acton, T.B., Xiao, R., Montelione, G.T., Aalberts, D.P., Hunt, J.F., 2016. Codon influence on protein expression in E. coli correlates with mRNA levels. Nature 529, 358-363. https://doi.org/10.1038/nature16509

Bogdan, S., Schultz, J., Grosshans, J., 2014. Formin' cellular structures. Commun Integr Biol 6. https://doi.org/10.4161/cib.27634

Bonello, T.T., Choi, W., Peifer, M., 2019. Scribble and discs-large direct initial assembly and positioning of adherens junctions during establishment of apical-basal polarity. Development. https://doi.org/10.1242/dev.180976 
Bonello, T.T., Perez-Vale, K.Z., Sumigray, K.D., Peifer, M., 2018. Rap1 acts via multiple mechanisms to position Canoe and adherens junctions and mediate apical-basal polarity establishment. Development 145. https://doi.org/10.1242/dev.157941

Bouissou, A., Vérollet, C., de Forges, H., Haren, L., Bellaïche, Y., Perez, F., Merdes, A., Raynaud-Messina, B., 2014. $\gamma$-Tubulin Ring Complexes and EB1 play antagonistic roles in microtubule dynamics and spindle positioning. EMBO J. 33, 114-128. https://doi.org/10.1002/embj.201385967

Brendza, R.P., Serbus, L.R., Duffy, J.B., Saxton, W.M., 2000. A function for kinesin I in the posterior transport of oskar mRNA and Staufen protein. Science 289, 2120-2122. https://doi.org/10.1126/science.289.5487.2120

Brendza, R.P., Serbus, L.R., Saxton, W.M., Duffy, J.B., 2002. Posterior localization of dynein and dorsalventral axis formation depend on kinesin in Drosophila oocytes. Current biology 12, 1541-1545.

Breuzard, G., Hubert, P., Nouar, R., De Bessa, T., Devred, F., Barbier, P., Sturgis, J.N., Peyrot, V., 2013. Molecular mechanisms of Tau binding to microtubules and its role in microtubule dynamics in live cells. Journal of Cell Science 126, 2810-2819. https://doi.org/10.1242/jcs.120832

Burke, T.A., Christensen, J.R., Barone, E., Suarez, C., Sirotkin, V., Kovar, D.R., 2014. Homeostatic actin cytoskeleton networks are regulated by assembly factor competition for monomers. Curr. Biol. 24, 579-585. https://doi.org/10.1016/j.cub.2014.01.072

Cai, Q., Gerwin, C., Sheng, Z.-H., 2005. Syntabulin-mediated anterograde transport of mitochondria along neuronal processes. The Journal of Cell Biology 170, 959-969. https://doi.org/10.1083/jcb.200506042

Cajigas, I.J., Tushev, G., Will, T.J., tom Dieck, S., Fuerst, N., Schuman, E.M., 2012. The local transcriptome in the synaptic neuropil revealed by deep sequencing and high-resolution imaging. Neuron 74, 453-466. https://doi.org/10.1016/j.neuron.2012.02.036

Calero-Cuenca, F.J., Espinosa-Vázquez, J.M., Reina-Campos, M., Díaz-Meco, M.T., Moscat, J., Sotillos, S., 2016. Nuclear fallout provides a new link between aPKC and polarized cell trafficking. BMC Biol 14. https://doi.org/10.1186/s12915-016-0253-6

Callan-Jones, A.C., Voituriez, R., 2016. Actin flows in cell migration: from locomotion and polarity to trajectories. Curr. Opin. Cell Biol. 38, 12-17. https://doi.org/10.1016/j.ceb.2016.01.003

Campenot, R.B., Eng, H., 2000. Protein synthesis in axons and its possible functions. J Neurocytol 29, $793-$ 798. https://doi.org/10.1023/A:1010939307434

Castrillon, D.H., Wasserman, S.A., 1994. Diaphanous is required for cytokinesis in Drosophila and shares domains of similarity with the products of the limb deformity gene. Development 120, 33673377.

Cheeks, R.J., Canman, J.C., Gabriel, W.N., Meyer, N., Strome, S., Goldstein, B., 2004. C. elegans PAR proteins function by mobilizing and stabilizing asymmetrically localized protein complexes. Curr. Biol. 14, 851-862. https://doi.org/10.1016/j.cub.2004.05.022

Choi, W., Harris, N.J., Sumigray, K.D., Peifer, M., 2013. Rap1 and Canoe/afadin are essential for establishment of apical-basal polarity in the Drosophila embryo. Mol Biol Cell 24, 945-963. https://doi.org/10.1091/mbc.E12-10-0736

Clark, A.G., Wartlick, O., Salbreux, G., Paluch, E.K., 2014. Stresses at the Cell Surface during Animal Cell Morphogenesis. Current Biology 24, R484-R494. https://doi.org/10.1016/j.cub.2014.03.059

Coles, C.H., Bradke, F., 2015. Coordinating Neuronal Actin-Microtubule Dynamics. Current Biology 25, R677-R691. https://doi.org/10.1016/j.cub.2015.06.020

Conduit, P.T., Wainman, A., Raff, J.W., 2015. Centrosome function and assembly in animal cells. Nat. Rev. Mol. Cell Biol. 16, 611-624. https://doi.org/10.1038/nrm4062

Cooper, J.A., 1987. Effects of cytochalasin and phalloidin on actin. J. Cell Biol. 105, 1473-1478. https://doi.org/10.1083/jcb.105.4.1473

Cooper, J.A., Pollard, T.D., 1985. Effect of capping protein on the kinetics of actin polymerization. Biochemistry 24, 793-799. https://doi.org/10.1021/bi00324a039

Coravos, J.S., Martin, A.C., 2016. Apical Sarcomere-like Actomyosin Contracts Nonmuscle Drosophila Epithelial Cells. Developmental Cell 39, 346-358. https://doi.org/10.1016/j.devcel.2016.09.023

Courtemanche, N., Pollard, T.D., 2013. Interaction of profilin with the barbed end of actin filaments. Biochemistry 52, 6456-6466. https://doi.org/10.1021/bi400682n

Cowan, C.R., Hyman, A.A., 2004. Centrosomes direct cell polarity independently of microtubule assembly in C. elegans embryos. Nature 431, 92-96. https://doi.org/10.1038/nature02825 
Craig, R., Woodhead, J.L., 2006. Structure and function of myosin filaments. Current Opinion in Structural Biology, Theory and simulation/Macromolecular assemblages 16, 204-212. https://doi.org/10.1016/j.sbi.2006.03.006

Cuenca, A.A., Schetter, A., Aceto, D., Kemphues, K., Seydoux, G., 2003. Polarization of the C. elegans zygote proceeds via distinct establishment and maintenance phases. Development 130, 12551265. https://doi.org/10.1242/dev.00284

Davies, S.P., Reddy, H., Caivano, M., Cohen, P., 2000. Specificity and mechanism of action of some commonly used protein kinase inhibitors. Biochem J 351, 95-105.

Deka, J., Kuhlmann, J., Müller, O., 1998. A domain within the tumor suppressor protein APC shows very similar biochemical properties as the microtubule-associated protein tau. Eur. J. Biochem. 253, 591-597. https://doi.org/10.1046/j.1432-1327.1998.2530591.x

Delevoye, C., Miserey-Lenkei, S., Montagnac, G., Gilles-Marsens, F., Paul-Gilloteaux, P., Giordano, F., Waharte, F., Marks, M.S., Goud, B., Raposo, G., 2014. Recycling endosome tubule morphogenesis from sorting endosomes requires the kinesin motor KIF13A. Cell Rep 6, 445-454. https://doi.org/10.1016/j.celrep.2014.01.002

Démoulin, D., Carlier, M.-F., Bibette, J., Baudry, J., 2014. Power transduction of actin filaments ratcheting in vitro against a load. PNAS 111, 17845-17850. https://doi.org/10.1073/pnas.1414184111

Desai, A., Mitchison, T.J., 1997. Microtubule Polymerization Dynamics. Annual Review of Cell and Developmental Biology 13, 83-117. https://doi.org/10.1146/annurev.cellbio.13.1.83

Dixit, R., Barnett, B., Lazarus, J.E., Tokito, M., Goldman, Y.E., Holzbaur, E.L.F., 2009. Microtubule plus-end tracking by CLIP-170 requires EB1. Proc. Natl. Acad. Sci. U.S.A. 106, 492-497. https://doi.org/10.1073/pnas.0807614106

Dogterom, M., Koenderink, G.H., 2019a. Actin-microtubule crosstalk in cell biology. Nat Rev Mol Cell Biol 20, 38-54. https://doi.org/10.1038/s41580-018-0067-1

Dogterom, M., Koenderink, G.H., 2019b. Actin-microtubule crosstalk in cell biology. Nat Rev Mol Cell Biol 20, 38-54. https://doi.org/10.1038/s41580-018-0067-1

Dominguez, R., Holmes, K.C., 2011. Actin structure and function. Annu Rev Biophys 40, 169-186. https://doi.org/10.1146/annurev-biophys-042910-155359

Driever, W., Nüsslein-Volhard, C., 1989. The bicoid protein is a positive regulator of hunchback transcription in the early Drosophila embryo. Nature 337, 138-143. https://doi.org/10.1038/337138a0

Du, W., Su, Q.P., 2019. Single-molecule in vitro reconstitution assay for kinesin-1-driven membrane dynamics. Biophys Rev 11, 319-325. https://doi.org/10.1007/s12551-019-00531-4

Edwards, M., Zwolak, A., Schafer, D.A., Sept, D., Dominguez, R., Cooper, J.A., 2014a. Capping protein regulators fine-tune actin assembly dynamics. Nat. Rev. Mol. Cell Biol. 15, 677-689. https://doi.org/10.1038/nrm3869

Edwards, M., Zwolak, A., Schafer, D.A., Sept, D., Dominguez, R., Cooper, J.A., 2014b. Capping protein regulators fine-tune actin assembly dynamics. Nature reviews Molecular cell biology 15, 677.

Ellis, R.J., 2001. Macromolecular crowding: obvious but underappreciated. Trends Biochem. Sci. 26, 597604. https://doi.org/10.1016/s0968-0004(01)01938-7

Ferrandon, D., Elphick, L., Nüsslein-Volhard, C., St Johnston, D., 1994. Staufen protein associates with the 3'UTR of bicoid mRNA to form particles that move in a microtubule-dependent manner. Cell 79, 1221-1232. https://doi.org/10.1016/0092-8674(94)90013-2

Feuge, J., Scharkowski, F., Michaelsen-Preusse, K., Korte, M., 2019. FMRP Modulates Activity-Dependent Spine Plasticity by Binding Cofilin1 mRNA and Regulating Localization and Local Translation. Cereb. Cortex. https://doi.org/10.1093/cercor/bhz059

Fiehler, R.W., Wolff, T., 2007. Drosophila Myosin II, Zipper, is Essential for Ommatidial Rotation. Dev Biol 310, 348-362. https://doi.org/10.1016/j.ydbio.2007.08.001

Fradin, C., 2017. On the importance of protein diffusion in biological systems: The example of the Bicoid morphogen gradient. Biochimica et Biophysica Acta (BBA) - Proteins and Proteomics, Biophysics in Canada 1865, 1676-1686. https://doi.org/10.1016/j.bbapap.2017.09.002

Fraschini, R., 2017. Factors that Control Mitotic Spindle Dynamics. Adv. Exp. Med. Biol. 925, 89-101. https://doi.org/10.1007/5584_2016_74

Fullilove, S.L., Jacobson, A.G., 1971. Nuclear elongation and cytokinesis in Drosophila montana. Dev. Biol. 26, 560-577. https://doi.org/10.1016/0012-1606(71)90141-2 
Gallaud, E., Caous, R., Pascal, A., Bazile, F., Gagné, J.-P., Huet, S., Poirier, G.G., Chrétien, D., RichardParpaillon, L., Giet, R., 2014. Ensconsin/Map7 promotes microtubule growth and centrosome separation in Drosophila neural stem cells. J. Cell Biol. 204, 1111-1121. https://doi.org/10.1083/jcb.201311094

Gáspár, I., Sysoev, V., Komissarov, A., Ephrussi, A., 2017. An RNA-binding atypical tropomyosin recruits kinesin-1 dynamically to oskar mRNPs. The EMBO Journal 36, 319-333. https://doi.org/10.15252/embj.201696038

Geisbrecht, E.R., Haralalka, S., Swanson, S.K., Florens, L., Washburn, M.P., Abmayr, S.M., 2008. Drosophila ELMO/CED-12 interacts with Myoblast city to direct myoblast fusion and ommatidial organization. Developmental Biology 314, 137-149. https://doi.org/10.1016/j.ydbio.2007.11.022

Giles, R.H., van Es, J.H., Clevers, H., 2003. Caught up in a Wnt storm: Wnt signaling in cancer. Biochimica et Biophysica Acta (BBA) - Reviews on Cancer 1653, 1-24. https://doi.org/10.1016/S0304419X(03)00005-2

Goldenring, J.R., 2015. Recycling endosomes. Current Opinion in Cell Biology, Cell organelles 35, 117-122. https://doi.org/10.1016/j.ceb.2015.04.018

Golenkina, S., Chaturvedi, V., Saint, R., Murray, M.J., 2018. Frazzled can act through distinct molecular pathways in epithelial cells to regulate motility, apical constriction, and localisation of E-Cadherin. PLoS ONE 13, e0194003. https://doi.org/10.1371/journal.pone.0194003

Gonsalvez, G.B., Urbinati, C.R., Long, R.M., 2005. RNA localization in yeast: moving towards a mechanism. Biol. Cell 97, 75-86. https://doi.org/10.1042/BC20040066

Goodwin, S.S., Vale, R.D., 2010. Patronin regulates the microtubule network by protecting microtubule minus ends. Cell 143, 263-274. https://doi.org/10.1016/j.cell.2010.09.022

Großhans, J., Wenzl, C., Herz, H.-M., Bartoszewski, S., Schnorrer, F., Vogt, N., Schwarz, H., Müller, H.-A., 2005. RhoGEF2 and the formin Dia control the formation of the furrow canal by directed actin assembly during Drosophila cellularisation. Development 132, 1009-1020. https://doi.org/10.1242/dev.01669

Guger, K.A., Gumbiner, B.M., 2000. A mode of regulation of beta-catenin signaling activity in Xenopus embryos independent of its levels. Dev. Biol. 223, 441-448. https://doi.org/10.1006/dbio.2000.9770

Hall, B.K., 1998. Evolutionary Developmental Biology. Springer Science \& Business Media.

Hanson, G., Coller, J., 2018. Codon optimality, bias and usage in translation and mRNA decay. Nature Reviews Molecular Cell Biology 19, 20-30. https://doi.org/10.1038/nrm.2017.91

Harigaya, Y., Parker, R., 2016. Analysis of the association between codon optimality and mRNA stability in Schizosaccharomyces pombe. BMC Genomics 17, 895. https://doi.org/10.1186/s12864-016-32376

Harris, T.J.C., Peifer, M., 2004. Adherens junction-dependent and -independent steps in the establishment of epithelial cell polarity in Drosophila. J. Cell Biol. 167, 135-147. https://doi.org/10.1083/jcb.200406024

Harris, T.J.C., Sawyer, J.K., Peifer, M., 2009. Chapter 3 How the Cytoskeleton Helps Build the Embryonic Body Plan: Models of Morphogenesis from Drosophila, in: Current Topics in Developmental Biology, Current Topics in Developmental Biology. Academic Press, pp. 55-85. https://doi.org/10.1016/S0070-2153(09)89003-0

Haruta, T., Warrior, R., Yonemura, S., Oda, H., 2010. The proximal half of the Drosophila E-cadherin extracellular region is dispensable for many cadherin-dependent events but required for ventral furrow formation. Genes Cells 15, 193-208. https://doi.org/10.1111/j.1365-2443.2010.01389.x

He, B., Martin, A., Wieschaus, E., 2016. Flow-dependent myosin recruitment during Drosophila cellularization requires zygotic dunk activity. Development 143, 2417-2430. https://doi.org/10.1242/dev.131334

He, Y., Francis, F., Myers, K.A., Yu, W., Black, M.M., Baas, P.W., 2005. Role of cytoplasmic dynein in the axonal transport of microtubules and neurofilaments. J. Cell Biol. 168, 697-703. https://doi.org/10.1083/jcb.200407191

Heber, S., Gáspár, I., Tants, J.-N., Günther, J., Moya, S.M.F., Janowski, R., Ephrussi, A., Sattler, M., Niessing, D., 2019. Staufen2-mediated RNA recognition and localization requires combinatorial action of multiple domains. Nat Commun 10, 1659. https://doi.org/10.1038/s41467-019-09655-3

Heng, Y.-W., Koh, C.-G., 2010. Actin cytoskeleton dynamics and the cell division cycle. Int. J. Biochem. Cell 
Biol. 42, 1622-1633. https://doi.org/10.1016/j.biocel.2010.04.007

Henty-Ridilla, J.L., Rankova, A., Eskin, J.A., Kenny, K., Goode, B.L., 2016. Accelerated actin filament polymerization from microtubule plus ends. Science 352, 1004-1009. https://doi.org/10.1126/science.aaf1709

Hickson, G.R.X., Matheson, J., Riggs, B., Maier, V.H., Fielding, A.B., Prekeris, R., Sullivan, W., Barr, F.A., Gould, G.W., 2003. Arfophilins Are Dual Arf/Rab 11 Binding Proteins That Regulate Recycling Endosome Distribution and Are Related to Drosophila Nuclear Fallout. Mol Biol Cell 14, 29082920. https://doi.org/10.1091/mbc.E03-03-0160

Higashida, C., Miyoshi, T., Fujita, A., Oceguera-Yanez, F., Monypenny, J., Andou, Y., Narumiya, S., Watanabe, N., 2004. Actin polymerization-driven molecular movement of mDia1 in living cells. Science 303, 2007-2010. https://doi.org/10.1126/science.1093923

Hirokawa, N., 1998. Kinesin and Dynein Superfamily Proteins and the Mechanism of Organelle Transport. Science 279, 519-526. https://doi.org/10.1126/science.279.5350.519

Hirokawa, N., 1982. Cross-linker system between neurofilaments, microtubules, and membranous organelles in frog axons revealed by the quick-freeze, deep-etching method. J. Cell Biol. 94, 129142. https://doi.org/10.1083/jcb.94.1.129

Hirokawa, N., Noda, Y., Tanaka, Y., Niwa, S., 2009. Kinesin superfamily motor proteins and intracellular transport. Nat. Rev. Mol. Cell Biol. 10, 682-696. https://doi.org/10.1038/nrm2774

Holt, C.E., Schuman, E.M., 2013. The central dogma decentralized: new perspectives on RNA function and local translation in neurons. Neuron 80,648-657. https://doi.org/10.1016/j.neuron.2013.10.036

Horne-Badovinac, S., Bilder, D., 2008. Dynein regulates epithelial polarity and the apical localization of stardust A mRNA. PLoS Genet. 4, e8. https://doi.org/10.1371/journal.pgen.0040008

Hotta, A., Kawakatsu, T., Nakatani, T., Sato, T., Matsui, C., Sukezane, T., Akagi, T., Hamaji, T., Grigoriev, I., Akhmanova, A., Takai, Y., Mimori-Kiyosue, Y., 2010. Laminin-based cell adhesion anchors microtubule plus ends to the epithelial cell basal cortex through LL5alpha/beta. J. Cell Biol. 189, 901-917. https://doi.org/10.1083/jcb.200910095

Hovland, R., Hesketh, J.E., Pryme, I.F., 1996. The compartmentalization of protein synthesis: importance of cytoskeleton and role in mRNA targeting. Int. J. Biochem. Cell Biol. 28, 1089-1105. https://doi.org/10.1016/1357-2725(96)00059-3

Hunter, A.W., Caplow, M., Coy, D.L., Hancock, W.O., Diez, S., Wordeman, L., Howard, J., 2003. The kinesinrelated protein MCAK is a microtubule depolymerase that forms an ATP-hydrolyzing complex at microtubule ends. Mol. Cell 11, 445-457.

Hutterer, A., Betschinger, J., Petronczki, M., Knoblich, J.A., 2004. Sequential roles of Cdc42, Par-6, aPKC, and $\mathrm{Lgl}$ in the establishment of epithelial polarity during Drosophila embryogenesis. Dev. Cell 6, 845-854. https://doi.org/10.1016/j.devcel.2004.05.003

Imai, K., Nakai, K., 2010. Prediction of subcellular locations of proteins: where to proceed? Proteomics 10, 3970-3983. https://doi.org/10.1002/pmic.201000274

Ishizaki, T., Maekawa, M., Fujisawa, K., Okawa, K., Iwamatsu, A., Fujita, A., Watanabe, N., Saito, Y., Kakizuka, A., Morii, N., Narumiya, S., 1996. The small GTP-binding protein Rho binds to and activates a $160 \mathrm{kDa}$ Ser/Thr protein kinase homologous to myotonic dystrophy kinase. EMBO J 15, 1885-1893.

Itoh, T., Erdmann, K.S., Roux, A., Habermann, B., Werner, H., De Camilli, P., 2005. Dynamin and the Actin Cytoskeleton Cooperatively Regulate Plasma Membrane Invagination by BAR and F-BAR Proteins. Developmental Cell 9, 791-804. https://doi.org/10.1016/j.devcel.2005.11.005

Iyer, K.V., Piscitello-Gómez, R., Paijmans, J., Jülicher, F., Eaton, S., 2019. Epithelial Viscoelasticity Is Regulated by Mechanosensitive E-cadherin Turnover. Curr. Biol. 29, 578-591.e5. https://doi.org/10.1016/j.cub.2019.01.021

Izaddoost, S., Nam, S.-C., Bhat, M.A., Bellen, H.J., Choi, K.-W., 2002. Drosophila Crumbs is a positional cue in photoreceptor adherens junctions and rhabdomeres. Nature 416, 178-183. https://doi.org/10.1038/nature720

Jambhekar, A., McDermott, K., Sorber, K., Shepard, K.A., Vale, R.D., Takizawa, P.A., DeRisi, J.L., 2005. Unbiased selection of localization elements reveals cis-acting determinants of mRNA bud localization in Saccharomyces cerevisiae. Proc. Natl. Acad. Sci. U.S.A. 102, 18005-18010. https://doi.org/10.1073/pnas.0509229102

Janson, M.E., de Dood, M.E., Dogterom, M., 2003. Dynamic instability of microtubules is regulated by 
force. J Cell Biol 161, 1029-1034. https://doi.org/10.1083/jcb.200301147

Ji, L., Lu, B., Zamponi, R., Charlat, O., Aversa, R., Yang, Z., Sigoillot, F., Zhu, X., Hu, T., Reece-Hoyes, J.S., Russ, C., Michaud, G., Tchorz, J.S., Jiang, X., Cong, F., 2019. USP7 inhibits Wnt/ $\beta$-catenin signaling through promoting stabilization of Axin. Nat Commun 10, 4184. https://doi.org/10.1038/s41467019-12143-3

Jimbo, T., Kawasaki, Y., Koyama, R., Sato, R., Takada, S., Haraguchi, K., Akiyama, T., 2002. Identification of a link between the tumour suppressor APC and the kinesin superfamily. Nat. Cell Biol. 4, 323-327. https://doi.org/10.1038/ncb779

Juanes, M.A., Isnardon, D., Badache, A., Brasselet, S., Mavrakis, M., Goode, B.L., 2019. The role of APCmediated actin assembly in microtubule capture and focal adhesion turnover. J. Cell Biol. 218, 3415-3435. https://doi.org/10.1083/jcb.201904165

Kabsch, W., Mannherz, H.G., Suck, D., Pai, E.F., Holmes, K.C., 1990. Atomic structure of the actin:DNase I complex. Nature 347, 37-44. https://doi.org/10.1038/347037a0

Kamm, K.E., Stull, J.T., 2001. Dedicated myosin light chain kinases with diverse cellular functions. J. Biol. Chem. 276, 4527-4530. https://doi.org/10.1074/jbc.R000028200

Kanda, T., Sullivan, K.F., Wahl, G.M., 1998. Histone-GFP fusion protein enables sensitive analysis of chromosome dynamics in living mammalian cells. Curr. Biol. 8, 377-385.

Kao, L.-R., Megraw, T.L., 2009. Centrocortin cooperates with centrosomin to organize Drosophila embryonic cleavage furrows. Curr. Biol. 19, 937-942. https://doi.org/10.1016/j.cub.2009.04.037

Kapitein, L.C., Peterman, E.J., Kwok, B.H., Kim, J.H., Kapoor, T.M., Schmidt, C.F., 2005. The bipolar mitotic kinesin Eg5 moves on both microtubules that it crosslinks. Nature 435, 114.

Karagiosis, S.A., Ready, D.F., 2004. Moesin contributes an essential structural role in Drosophila photoreceptor morphogenesis. Development 131, 725-732. https://doi.org/10.1242/dev.00976

Karess, R.E., Chang, X.J., Edwards, K.A., Kulkarni, S., Aguilera, I., Kiehart, D.P., 1991. The regulatory light chain of nonmuscle myosin is encoded by spaghetti-squash, a gene required for cytokinesis in Drosophila. Cell 65, 1177-1189. https://doi.org/10.1016/0092-8674(91)90013-o

Karr, T.L., Alberts, B.M., 1986. Organization of the cytoskeleton in early Drosophila embryos. J. Cell Biol. 102, 1494-1509. https://doi.org/10.1083/jcb.102.4.1494

Kashida, S., Wang, D.O., Saito, H., Gueroui, Z., 2019. Nanoparticle-based local translation reveals mRNA as a translation-coupled scaffold with anchoring function. Proc. Natl. Acad. Sci. U.S.A. 116, 1334613351. https://doi.org/10.1073/pnas.1900310116

Kawasaki, Y., Senda, T., Ishidate, T., Koyama, R., Morishita, T., Iwayama, Y., Higuchi, O., Akiyama, T., 2000. Asef, a link between the tumor suppressor APC and G-protein signaling. Science 289, 1194-1197. https://doi.org/10.1126/science.289.5482.1194

Khanal, I., Elbediwy, A., Loza, M. del C.D. de la, Fletcher, G.C., Thompson, B.J., 2016. Shot and Patronin polarise microtubules to direct membrane traffic and biogenesis of microvilli in epithelia. J Cell Sci 129, 2651-2659. https://doi.org/10.1242/jcs.189076

Kim-Ha, J., Smith, J.L., Macdonald, P.M., 1991. oskar mRNA is localized to the posterior pole of the Drosophila oocyte. Cell 66, 23-35. https://doi.org/10.1016/0092-8674(91)90136-m

Kosako, H., Goto, H., Yanagida, M., Matsuzawa, K., Fujita, M., Tomono, Y., Okigaki, T., Odai, H., Kaibuchi, K., Inagaki, M., 1999. Specific accumulation of Rho-associated kinase at the cleavage furrow during cytokinesis: cleavage furrow-specific phosphorylation of intermediate filaments. Oncogene 18, 2783-2788. https://doi.org/10.1038/sj.onc.1202633

Kovar, D.R., Pollard, T.D., 2004. Insertional assembly of actin filament barbed ends in association with formins produces piconewton forces. Proc. Natl. Acad. Sci. U.S.A. 101, 14725-14730. https://doi.org/10.1073/pnas.0405902101

Krueger, D., Tardivo, P., Nguyen, C., De Renzis, S., 2018. Downregulation of basal myosin-II is required for cell shape changes and tissue invagination. EMBO J 37. https://doi.org/10.15252/embj.2018100170

Kugler, J.-M., Lasko, P., 2009. Localization, anchoring and translational control of oskar, gurken, bicoid and nanos mRNA during Drosophila oogenesis. Fly (Austin) 3, 15-28. https://doi.org/10.4161/fly.3.1.7751

Kumfer, K.T., Cook, S.J., Squirrell, J.M., Eliceiri, K.W., Peel, N., O'Connell, K.F., White, J.G., 2010. CGEF-1 and CHIN-1 Regulate CDC-42 Activity during Asymmetric Division in the Caenorhabditis elegans Embryo. Mol Biol Cell 21, 266-277. https://doi.org/10.1091/mbc.E09-01-0060 
Lawrence, P.A., 1988. Background to bicoid. Cell 54, 1-2. https://doi.org/10.1016/0092-8674(88)90172-9

League, G.P., Nam, S.-C., 2011. Role of Kinesin Heavy Chain in Crumbs Localization along the Rhabdomere Elongation in Drosophila Photoreceptor. PLOS ONE 6, e21218. https://doi.org/10.1371/journal.pone.0021218

Lecuit, T., Samanta, R., Wieschaus, E., 2002. slam encodes a developmental regulator of polarized membrane growth during cleavage of the Drosophila embryo. Dev. Cell 2, 425-436.

Lehmann, R., Nüsslein-Volhard, C., 1986. Abdominal segmentation, pole cell formation, and embryonic polarity require the localized activity of oskar, a maternal gene in Drosophila. Cell 47, 141-152. https://doi.org/10.1016/0092-8674(86)90375-2

Ling, J., Umezawa, K.Y., Scott, T., Small, S., 2019. Bicoid-Dependent Activation of the Target Gene hunchback Requires a Two-Motif Sequence Code in a Specific Basal Promoter. Mol. Cell 75, 11781187.e4. https://doi.org/10.1016/j.molcel.2019.06.038

Loria, A., Longhini, K.M., Glotzer, M., 2012. The RhoGAP domain of CYK-4 has an essential role in RhoA activation. Curr. Biol. 22, 213-219. https://doi.org/10.1016/j.cub.2011.12.019

Lu, W., Winding, M., Lakonishok, M., Wildonger, J., Gelfand, V.I., 2016. Microtubule-microtubule sliding by kinesin-1 is essential for normal cytoplasmic streaming in Drosophila oocytes. Proc Natl Acad Sci USA 113, E4995-E5004. https://doi.org/10.1073/pnas.1522424113

Lv, Z., Großhans, J., 2016. A Radial Actin Network in Apical Constriction. Developmental Cell 39, $280-282$. https://doi.org/10.1016/j.devcel.2016.10.017

Madaule, P., Eda, M., Watanabe, N., Fujisawa, K., Matsuoka, T., Bito, H., Ishizaki, T., Narumiya, S., 1998. Role of citron kinase as a target of the small GTPase Rho in cytokinesis. Nature 394, 491-494. https://doi.org/10.1038/28873

Manchalu, S., Mittal, N., Spang, A., Jansen, R.P., 2019. Local translation of yeast ERG4 mRNA at the endoplasmic reticulum requires the brefeldin $A$ resistance protein Bfr1. RNA. https://doi.org/10.1261/rna.072017.119

Manning, L.A., Perez-Vale, K.Z., Schaefer, K.N., Sewell, M.T., Peifer, M., 2019. The Drosophila Afadin and ZO-1 homologues Canoe and Polychaetoid act in parallel to maintain epithelial integrity when challenged by adherens junction remodeling. Mol Biol Cell 30, 1938-1960. https://doi.org/10.1091/mbc.E19-04-0209

Martin, A.C., Kaschube, M., Wieschaus, E.F., 2009. Pulsed actin-myosin network contractions drive apical constriction. Nature 457, 495. https://doi.org/10.1038/nature07522

Mavrakis, M., Rikhy, R., Lippincott-Schwartz, J., 2009. Plasma membrane polarity and compartmentalization are established before cellularization in the fly embryo. Dev. Cell 16, 93104. https://doi.org/10.1016/j.devcel.2008.11.003

Médina, E., Lemmers, C., Lane-Guermonprez, L., Le Bivic, A., 2002. Role of the Crumbs complex in the regulation of junction formation in Drosophila and mammalian epithelial cells. Biol. Cell 94, 305313.

Meer, E.J., Wang, D.O., Kim, S., Barr, I., Guo, F., Martin, K.C., 2012. Identification of a cis-acting element that localizes mRNA to synapses. Proc. Natl. Acad. Sci. U.S.A. 109, 4639-4644. https://doi.org/10.1073/pnas.1116269109

Meng, W., Mushika, Y., Ichii, T., Takeichi, M., 2008. Anchorage of microtubule minus ends to adherens junctions regulates epithelial cell-cell contacts. Cell 135, 948-959. https://doi.org/10.1016/j.cell.2008.09.040

Metzger, T., Gache, V., Xu, M., Cadot, B., Folker, E.S., Richardson, B.E., Gomes, E.R., Baylies, M.K., 2012. MAP and kinesin-dependent nuclear positioning is required for skeletal muscle function. Nature 484, 120.

Micklem, D.R., Adams, J., Grünert, S., St Johnston, D., 2000. Distinct roles of two conserved Staufen domains in oskar mRNA localization and translation. EMBO J. 19, 1366-1377. https://doi.org/10.1093/emboj/19.6.1366

Ming, G., Wong, S.T., Henley, J., Yuan, X., Song, H., Spitzer, N.C., Poo, M., 2002. Adaptation in the chemotactic guidance of nerve growth cones. Nature 417, 411-418. https://doi.org/10.1038/nature745

Morrison, E.E., 2009. The APC-EB1 interaction. Adv. Exp. Med. Biol. 656, 41-50. https://doi.org/10.1007/978-1-4419-1145-2_4

Müller, T., Hess, M.W., Schiefermeier, N., Pfaller, K., Ebner, H.L., Heinz-Erian, P., Ponstingl, H., Partsch, J., 
Röllinghoff, B., Köhler, H., Berger, T., Lenhartz, H., Schlenck, B., Houwen, R.J., Taylor, C.J., Zoller, H., Lechner, S., Goulet, O., Utermann, G., Ruemmele, F.M., Huber, L.A., Janecke, A.R., 2008. MYO5B mutations cause microvillus inclusion disease and disrupt epithelial cell polarity. Nature Genetics 40, 1163-1165. https://doi.org/10.1038/ng.225

Munemitsu, S., Souza, B., Müller, O., Albert, I., Rubinfeld, B., Polakis, P., 1994. The APC Gene Product Associates with Microtubules in Vivo and Promotes Their Assembly in Vitro. Cancer Res 54, 36763681.

Munro, E., Nance, J., Priess, J.R., 2004. Cortical flows powered by asymmetrical contraction transport PAR proteins to establish and maintain anterior-posterior polarity in the early C. elegans embryo. Dev. Cell 7, 413-424. https://doi.org/10.1016/j.devcel.2004.08.001

Nangaku, M., Sato-Yoshitake, R., Okada, Y., Noda, Y., Takemura, R., Yamazaki, H., Hirokawa, N., 1994. KIF1B, a novel microtubule plus end-directed monomeric motor protein for transport of mitochondria. Cell 79, 1209-1220. https://doi.org/10.1016/0092-8674(94)90012-4

Nashchekin, D., Fernandes, A.R., St Johnston, D., 2016. Patronin/Shot Cortical Foci Assemble the Noncentrosomal Microtubule Array that Specifies the Drosophila Anterior-Posterior Axis. Dev. Cell 38, 61-72. https://doi.org/10.1016/j.devcel.2016.06.010

Nejedla, M., Sadi, S., Sulimenko, V., de Almeida, F.N., Blom, H., Draber, P., Aspenström, P., Karlsson, R., 2016. Profilin connects actin assembly with microtubule dynamics. Mol Biol Cell 27, 2381-2393. https://doi.org/10.1091/mbc.E15-11-0799

Nieuwburg, R., Nashchekin, D., Jakobs, M., Carter, A.P., Khuc Trong, P., Goldstein, R.E., St Johnston, D., n.d. Localised dynactin protects growing microtubules to deliver oskar mRNA to the posterior cortex of the Drosophila oocyte. eLife 6. https://doi.org/10.7554/eLife.27237

Noordstra, I., Liu, Q., Nijenhuis, W., Hua, S., Jiang, K., Baars, M., Remmelzwaal, S., Martin, M., Kapitein, L.C., Akhmanova, A., 2016. Control of apico-basal epithelial polarity by the microtubule minusend-binding protein CAMSAP3 and spectraplakin ACF7. J. Cell. Sci. 129, 4278-4288. https://doi.org/10.1242/jcs.194878

Ono, Y., Urata, Y., Goto, S., Nakagawa, S., Humbert, P.O., Li, T.-S., Zammit, P.S., 2015. Muscle Stem Cell Fate Is Controlled by the Cell-Polarity Protein Scrib. Cell Reports 10, 1135-1148. https://doi.org/10.1016/j.celrep.2015.01.045

Palacios, I.M., St Johnston, D., 2002. Kinesin light chain-independent function of the Kinesin heavy chain in cytoplasmic streaming and posterior localisation in the Drosophila oocyte. Development 129, 5473-5485. https://doi.org/10.1242/dev.00119

Pan, X., Cao, Y., Stucchi, R., Hooikaas, P.J., Portegies, S., Will, L., Martin, M., Akhmanova, A., Harterink, M., Hoogenraad, C.C., 2019. MAP7D2 Localizes to the Proximal Axon and Locally Promotes Kinesin-1Mediated Cargo Transport into the Axon. Cell Rep 26, 1988-1999.e6. https://doi.org/10.1016/j.celrep.2019.01.084

Paul, A.S., Pollard, T.D., 2009. Review of the mechanism of processive actin filament elongation by formins. Cell Motil. Cytoskeleton 66, 606-617. https://doi.org/10.1002/cm.20379

Pellikka, M., Tanentzapf, G., Pinto, M., Smith, C., McGlade, C.J., Ready, D.F., Tepass, U., 2002. Crumbs, the Drosophila homologue of human CRB1/RP12, is essential for photoreceptor morphogenesis. Nature 416, 143-149. https://doi.org/10.1038/nature721

Pereira, G., Schiebel, E., 1997. Centrosome-microtubule nucleation. J. Cell. Sci. 110 ( Pt 3), $295-300$.

Perez, F., Diamantopoulos, G.S., Stalder, R., Kreis, T.E., 1999. CLIP-170 highlights growing microtubule ends in vivo. Cell 96, 517-527. https://doi.org/10.1016/s0092-8674(00)80656-x

Petry, S., 2016. Mechanisms of Mitotic Spindle Assembly. Annu. Rev. Biochem. 85, 659-683. https://doi.org/10.1146/annurev-biochem-060815-014528

Piekny, A.J., Mains, P.E., 2002. Rho-binding kinase (LET-502) and myosin phosphatase (MEL-11) regulate cytokinesis in the early Caenorhabditis elegans embryo. J. Cell. Sci. 115, 2271-2282.

Pilling, A.D., Horiuchi, D., Lively, C.M., Saxton, W.M., 2006. Kinesin-1 and Dynein Are the Primary Motors for Fast Transport of Mitochondria in Drosophila Motor Axons. MBoC 17, 2057-2068. https://doi.org/10.1091/mbc.e05-06-0526

Piper, M., Salih, S., Weinl, C., Holt, C.E., Harris, W.A., 2005. Endocytosis-dependent desensitization and protein synthesis-dependent resensitization in retinal growth cone adaptation. Nat. Neurosci. 8, 179-186. https://doi.org/10.1038/nn1380

Plant, P.J., Fawcett, J.P., Lin, D.C.C., Holdorf, A.D., Binns, K., Kulkarni, S., Pawson, T., 2003. A polarity 
complex of mPar-6 and atypical PKC binds, phosphorylates and regulates mammalian LgI. Nat Cell Biol 5, 301-308. https://doi.org/10.1038/ncb948

Polakis, P., 2000. Wnt signaling and cancer. Genes Dev. 14, $1837-1851$. https://doi.org/10.1101/gad.14.15.1837

Pollard, T.D., 2016. Actin and Actin-Binding Proteins. Cold Spring Harb Perspect Biol 8, a018226. https://doi.org/10.1101/cshperspect.a018226

Postner, M.A., Miller, K.G., Wieschaus, E.F., 1992. Maternal effect mutations of the sponge locus affect actin cytoskeletal rearrangements in Drosophila melanogaster embryos. The Journal of Cell Biology 119, 1205-1218. https://doi.org/10.1083/jcb.119.5.1205

Preciado López, M., Huber, F., Grigoriev, I., Steinmetz, M.O., Akhmanova, A., Koenderink, G.H., Dogterom, M., 2014. Actin-microtubule coordination at growing microtubule ends. Nat Commun 5, 4778. https://doi.org/10.1038/ncomms5778

Pring, M., Evangelista, M., Boone, C., Yang, C., Zigmond, S.H., 2003. Mechanism of formin-induced nucleation of actin filaments. Biochemistry 42, 486-496. https://doi.org/10.1021/bi026520j

Radhakrishnan, A., Chen, Y.-H., Martin, S., Alhusaini, N., Green, R., Coller, J., 2016. The DEAD-Box Protein Dhh1p Couples mRNA Decay and Translation by Monitoring Codon Optimality. Cell 167, 122132.e9. https://doi.org/10.1016/j.cell.2016.08.053

Raff, J.W., Glover, D.M., 1989a. Centrosomes, and not nuclei, initiate pole cell formation in Drosophila embryos. Cell 57, 611-619.

Raff, J.W., Glover, D.M., 1989b. Centrosomes, and not nuclei, initiate pole cell formation in Drosophila embryos. Cell 57, 611-619. https://doi.org/10.1016/0092-8674(89)90130-X

Ramjaun, A.R., Micheva, K.D., Bouchelet, I., McPherson, P.S., 1997. Identification and characterization of a nerve terminal-enriched amphiphysin isoform. J. Biol. Chem. 272, 16700-16706. https://doi.org/10.1074/jbc.272.26.16700

Rao, A.N., Patil, A., Black, M.M., Craig, E.M., Myers, K.A., Yeung, H.T., Baas, P.W., 2017. Cytoplasmic Dynein Transports Axonal Microtubules in a Polarity-Sorting Manner. Cell Rep 19, 2210-2219. https://doi.org/10.1016/j.celrep.2017.05.064

Riggs, B., Rothwell, W., Mische, S., Hickson, G.R.X., Matheson, J., Hays, T.S., Gould, G.W., Sullivan, W., 2003. Actin cytoskeleton remodeling during early Drosophila furrow formation requires recycling endosomal components Nuclear-fallout and Rab11. J Cell Biol 163, 143-154. https://doi.org/10.1083/jcb.200305115

Rikhy, R., Mavrakis, M., Lippincott-Schwartz, J., 2015. Dynamin regulates metaphase furrow formation and plasma membrane compartmentalization in the syncytial Drosophila embryo. Biol Open 4, 301-311. https://doi.org/10.1242/bio.20149936

Ross, J.L., Ali, M.Y., Warshaw, D.M., 2008. Cargo transport: molecular motors navigate a complex cytoskeleton. Curr. Opin. Cell Biol. 20, 41-47. https://doi.org/10.1016/j.ceb.2007.11.006

Rottner, K., Hänisch, J., Campellone, K.G., 2010. WASH, WHAMM and JMY: regulation of Arp2/3 complex and beyond. Trends in Cell Biology 20, 650-661. https://doi.org/10.1016/j.tcb.2010.08.014

Rouiller, I., Xu, X.-P., Amann, K.J., Egile, C., Nickell, S., Nicastro, D., Li, R., Pollard, T.D., Volkmann, N., Hanein, D., 2008. The structural basis of actin filament branching by the Arp2/3 complex. J Cell Biol 180, 887-895. https://doi.org/10.1083/jcb.200709092

Royer, C., Lu, X., 2011. Epithelial cell polarity: a major gatekeeper against cancer? Cell Death Differ 18, 1470-1477. https://doi.org/10.1038/cdd.2011.60

Royou, A., Field, C., Sisson, J.C., Sullivan, W., Karess, R., 2003. Reassessing the Role and Dynamics of Nonmuscle Myosin II during Furrow Formation in Early Drosophila Embryos. MBoC 15, 838-850. https://doi.org/10.1091/mbc.e03-06-0440

Sanchez, A.D., Feldman, J.L., 2017. Microtubule-organizing centers: from the centrosome to noncentrosomal sites. Curr Opin Cell Biol 44, 93-101. https://doi.org/10.1016/j.ceb.2016.09.003

Sanger, A., Yip, Y.Y., Randall, T.S., Pernigo, S., Steiner, R.A., Dodding, M.P., 2017. SKIP controls lysosome positioning using a composite kinesin-1 heavy and light chain-binding domain. J Cell Sci 130, 1637-1651.

Schimert, K.I., Budaitis, B.G., Reinemann, D.N., Lang, M.J., Verhey, K.J., 2019. Intracellular cargo transport by single-headed kinesin motors. Proc. Natl. Acad. Sci. U.S.A. 116, 6152-6161. https://doi.org/10.1073/pnas.1817924116

Schmidt, A., Grosshans, J., 2018. Dynamics of cortical domains in early Drosophila development. J Cell Sci 
$131, \mathrm{jcs} 212795$.

Schmidt, A., Lv, Z., Grols shans, J., 2018. ELMO and Sponge specify subapical restriction of Canoe and formation of the subapical domain in early Drosophila embryos. Development 145, dev157909.

Scholey, J.M., Taylor, K.A., Kendrick-Jones, J., 1980. Regulation of non-muscle myosin assembly by calmodulin-dependent light chain kinase. Nature 287, 233-235. https://doi.org/10.1038/287233a0

Schulze, E., Kirschner, M., 1986. Microtubule dynamics in interphase cells. J. Cell Biol. 102, 1020-1031. https://doi.org/10.1083/jcb.102.3.1020

Schuyler, S.C., Pellman, D., 2001. Microtubule "plus-end-tracking proteins": The end is just the beginning. Cell 105, 421-424. https://doi.org/10.1016/s0092-8674(01)00364-6

Sen, A., Nagy-Zsvér-Vadas, Z., Krahn, M.P., 2012. Drosophila PATJ supports adherens junction stability by modulating Myosin light chain activity. J. Cell Biol. 199, 685-698. https://doi.org/10.1083/jcb.201206064

Shahbazi, M.N., Megias, D., Epifano, C., Akhmanova, A., Gundersen, G.G., Fuchs, E., Perez-Moreno, M., 2013. CLASP2 interacts with p120-catenin and governs microtubule dynamics at adherens junctions. J. Cell Biol. 203, 1043-1061. https://doi.org/10.1083/jcb.201306019

Simpson-Brose, M., Treisman, J., Desplan, C., 1994. Synergy between the hunchback and bicoid morphogens is required for anterior patterning in Drosophila. Cell 78, 855-865. https://doi.org/10.1016/S0092-8674(94)90622-X

Smith, D.E., Gruenbaum, Y., Berrios, M., Fisher, P.A., 1987. Biosynthesis and interconversion of Drosophila nuclear lamin isoforms during normal growth and in response to heat shock. J. Cell Biol. 105, 771-790. https://doi.org/10.1083/jcb.105.2.771

Sokac, A.M., Wieschaus, E., 2008. Local actin-dependent endocytosis is zygotically controlled to initiate Drosophila cellularization. Dev. Cell 14, 775-786. https://doi.org/10.1016/j.devcel.2008.02.014

Somlyo, A.P., Somlyo, A.V., 2003. Ca2+ sensitivity of smooth muscle and nonmuscle myosin II: modulated by $G$ proteins, kinases, and myosin phosphatase. Physiol. Rev. 83, 1325-1358. https://doi.org/10.1152/physrev.00023.2003

Song, J.-G., King, M.R., Zhang, R., Kadzik, R.S., Thawani, A., Petry, S., 2018. Mechanism of how augmin directly targets the $\gamma$-tubulin ring complex to microtubules. J. Cell Biol. 217, 2417-2428. https://doi.org/10.1083/jcb.201711090

Sørensen, M.A., Pedersen, S., 1991. Absolute in vivo translation rates of individual codons in Escherichia coli: The two glutamic acid codons GAA and GAG are translated with a threefold difference in rate. Journal of Molecular Biology 222, 265-280. https://doi.org/10.1016/0022-2836(91)90211-N

Spector, I., Shochet, N.R., Kashman, Y., Groweiss, A., 1983. Latrunculins: novel marine toxins that disrupt microfilament organization in cultured cells. Science 219, 493-495. https://doi.org/10.1126/science.6681676

Spracklen, A.J., Fagan, T.N., Lovander, K.E., Tootle, T.L., 2014. The pros and cons of common actin labeling tools for visualizing actin dynamics during Drosophila oogenesis. Dev. Biol. 393, 209-226. https://doi.org/10.1016/j.ydbio.2014.06.022

Squire, J.M., 1972. General model of myosin filament structure. II. Myosin filaments and cross-bridge interactions in vertebrate striated and insect flight muscles. J. Mol. Biol. 72, 125-138. https://doi.org/10.1016/0022-2836(72)90074-5

St Johnston, D., 2005. Moving messages: the intracellular localization of mRNAs. Nat. Rev. Mol. Cell Biol. 6, 363-375. https://doi.org/10.1038/nrm1643

St Johnston, D., Beuchle, D., Nüsslein-Volhard, C., 1991. Staufen, a gene required to localize maternal RNAs in the Drosophila egg. Cell 66, 51-63. https://doi.org/10.1016/0092-8674(91)90138-o

Staal, F.J.T., van Noort, M., Strous, G.J., Clevers, H.C., 2002. Wnt signals are transmitted through Nterminally dephosphorylated beta-catenin. EMBO Rep. 3, 63-68. https://doi.org/10.1093/emboreports/kvf002

Stehbens, S.J., Paterson, A.D., Crampton, M.S., Shewan, A.M., Ferguson, C., Akhmanova, A., Parton, R.G., Yap, A.S., 2006. Dynamic microtubules regulate the local concentration of E-cadherin at cell-cell contacts. J. Cell. Sci. 119, 1801-1811. https://doi.org/10.1242/jcs.02903

Stein, J.A., Broihier, H.T., Moore, L.A., Lehmann, R., 2002. Slow as molasses is required for polarized membrane growth and germ cell migration in Drosophila. Development 129, 3925-3934.

Stepien, B.K., Oppitz, C., Gerlach, D., Dag, U., Novatchkova, M., Krüttner, S., Stark, A., Keleman, K., 2016. 
RNA-binding profiles of Drosophila CPEB proteins Orb and Orb2. PNAS 113, E7030-E7038. https://doi.org/10.1073/pnas.1603715113

Su, J., Chow, B., Boulianne, G.L., Wilde, A., 2013. The BAR domain of amphiphysin is required for cleavage furrow tip-tubule formation during cellularization in Drosophila embryos. Molecular biology of the cell 24, 1444-1453.

Su, L.-K., Burrell, M., Hill, D.E., Gyuris, J., Brent, R., Wiltshire, R., Trent, J., Vogelstein, B., Kinzler, K.W., 1995. APC Binds to the Novel Protein EB1. Cancer Res 55, 2972-2977.

Su, L.K., Burrell, M., Hill, D.E., Gyuris, J., Brent, R., Wiltshire, R., Trent, J., Vogelstein, B., Kinzler, K.W., 1995. APC binds to the novel protein EB1. Cancer Res. 55, 2972-2977.

Su, L.K., Vogelstein, B., Kinzler, K.W., 1993. Association of the APC tumor suppressor protein with catenins. Science 262, 1734-1737. https://doi.org/10.1126/science.8259519

Suarez, C., Carroll, R.T., Burke, T.A., Christensen, J.R., Bestul, A.J., Sees, J.A., James, M.L., Sirotkin, V., Kovar, D.R., 2015a. Profilin Regulates F-actin Network Homeostasis by Favoring Formin Over Arp2/3 Complex. Dev Cell 32, 43-53. https://doi.org/10.1016/j.devcel.2014.10.027

Suarez, C., Carroll, R.T., Burke, T.A., Christensen, J.R., Bestul, A.J., Sees, J.A., James, M.L., Sirotkin, V., Kovar, D.R., 2015b. Profilin regulates F-actin network homeostasis by favoring formin over Arp2/3 complex. Developmental cell 32, 43-53.

Sun, J., Stathopoulos, A., 2018. FGF controls epithelial-mesenchymal transitions during gastrulation by regulating cell division and apicobasal polarity. Development 145. https://doi.org/10.1242/dev.161927

Svitkina, T., 2018. The Actin Cytoskeleton and Actin-Based Motility. Cold Spring Harb Perspect Biol 10. https://doi.org/10.1101/cshperspect.a018267

Svitkina, T.M., Borisy, G.G., 1999. Arp2/3 Complex and Actin Depolymerizing Factor/Cofilin in Dendritic Organization and Treadmilling of Actin Filament Array in Lamellipodia. J Cell Biol 145, 1009-1026.

Takei, K., Slepnev, V.I., Haucke, V., De Camilli, P., 1999. Functional partnership between amphiphysin and dynamin in clathrin-mediated endocytosis. Nat. Cell Biol. 1, 33-39. https://doi.org/10.1038/9004

Taneja, N., Burnette, D.T., 2019. Myosin IIA drives membrane bleb retraction. Mol Biol Cell 30, 1051-1059. https://doi.org/10.1091/mbc.E18-11-0752

Tautz, D., 1988. Regulation of the Drosophila segmentation gene hunchback by two maternal morphogenetic centres. Nature 332, 281-284. https://doi.org/10.1038/332281a0

Taylor, A.M., Berchtold, N.C., Perreau, V.M., Tu, C.H., Jeon, N.L., Cotman, C.W., 2009. Axonal mRNA in Uninjured and Regenerating Cortical Mammalian Axons. J. Neurosci. 29, 4697-4707. https://doi.org/10.1523/JNEUROSCI.6130-08.2009

Tepass, U., Theres, C., Knust, E., 1990. crumbs encodes an EGF-like protein expressed on apical membranes of Drosophila epithelial cells and required for organization of epithelia. Cell 61, 787799. https://doi.org/10.1016/0092-8674(90)90189-I

Terada, S., 2003. Where does slow axonal transport go? Neuroscience Research 47, $367-372$. https://doi.org/10.1016/j.neures.2003.08.005

Terada, S., Kinjo, M., Aihara, M., Takei, Y., Hirokawa, N., 2010. Kinesin-1/Hsc70-dependent mechanism of slow axonal transport and its relation to fast axonal transport. The EMBO Journal 29, 843-854. https://doi.org/10.1038/emboj.2009.389

Thawani, A., Kadzik, R.S., Petry, S., 2018. XMAP215 is a microtubule nucleation factor that functions synergistically with the $\gamma$-tubulin ring complex. Nat. Cell Biol. 20, 575-585. https://doi.org/10.1038/s41556-018-0091-6

Thomas, G.H., Williams, J.A., 1999. Dynamic rearrangement of the spectrin membrane skeleton during the generation of epithelial polarity in Drosophila. J. Cell. Sci. 112 ( Pt 17), 2843-2852.

Toya, M., Kobayashi, S., Kawasaki, M., Shioi, G., Kaneko, M., Ishiuchi, T., Misaki, K., Meng, W., Takeichi, M., 2016. CAMSAP3 orients the apical-to-basal polarity of microtubule arrays in epithelial cells. Proc. Natl. Acad. Sci. U.S.A. 113, 332-337. https://doi.org/10.1073/pnas.1520638113

Trovisco, V., Belaya, K., Nashchekin, D., Irion, U., Sirinakis, G., Butler, R., Lee, J.J., Gavis, E.R., St Johnston, D., 2016. bicoid mRNA localises to the Drosophila oocyte anterior by random Dynein-mediated transport and anchoring. Elife 5. https://doi.org/10.7554/eLife.17537

Van Horck, F.P.G., Holt, C.E., 2008. A cytoskeletal platform for local translation in axons. Sci Signal 1, pe11. https://doi.org/10.1126/stke.18pe11

Varshney, N., Sanyal, K., 2019. Nuclear migration in budding yeasts: position before division. Curr. Genet. 
https://doi.org/10.1007/s00294-019-01000-x

Velle, K.B., Fritz-Laylin, L.K., 2019. Diversity and evolution of actin-dependent phenotypes. Current Opinion in Genetics \& Development 58-59, 40-48. https://doi.org/10.1016/j.gde.2019.07.016

Volkov, V.A., Huis in 't Veld, P.J., Dogterom, M., Musacchio, A., n.d. Multivalency of NDC80 in the outer kinetochore is essential to track shortening microtubules and generate forces. elife 7. https://doi.org/10.7554/eLife.36764

Wagner, O.I., Ascaño, J., Tokito, M., Leterrier, J.-F., Janmey, P.A., Holzbaur, E.L.F., 2004. The interaction of neurofilaments with the microtubule motor cytoplasmic dynein. Mol. Biol. Cell 15, 5092-5100. https://doi.org/10.1091/mbc.e04-05-0401

Warn, R.M., Bullard, B., Magrath, R., 1980. Changes in the distribution of cortical myosin during the cellularization of the Drosophila embryo. J Embryol Exp Morphol 57, 167-176.

Warn, R.M., Magrath, R., Webb, S., 1984. Distribution of F-actin during cleavage of the Drosophila syncytial blastoderm. The Journal of Cell Biology 98, 156-162. https://doi.org/10.1083/jcb.98.1.156

Watanabe, S., Ando, Y., Yasuda, S., Hosoya, H., Watanabe, N., Ishizaki, T., Narumiya, S., 2008. mDia2 induces the actin scaffold for the contractile ring and stabilizes its position during cytokinesis in NIH 3 T3 cells. Mol. Biol. Cell 19, 2328-2338. https://doi.org/10.1091/mbc.e07-10-1086

Watanabe, T., Wang, S., Noritake, J., Sato, K., Fukata, M., Takefuji, M., Nakagawa, M., Izumi, N., Akiyama, T., Kaibuchi, K., 2004. Interaction with IQGAP1 links APC to Rac1, Cdc42, and actin filaments during cell polarization and migration. Dev. Cell 7, 871-883. https://doi.org/10.1016/j.devcel.2004.10.017

Webb, R.L., Zhou, M.-N., McCartney, B.M., 2009. A novel role for an APC2-Diaphanous complex in regulating actin organization in Drosophila. Development 136, 1283-1293. https://doi.org/10.1242/dev.026963

Wei, Z., Li, Y., Ye, F., Zhang, M., 2015. Structural basis for the phosphorylation-regulated interaction between the cytoplasmic tail of cell polarity protein crumbs and the actin-binding protein moesin. J. Biol. Chem. 290, 11384-11392. https://doi.org/10.1074/jbc.M115.643791

Weingarten, M.D., Lockwood, A.H., Hwo, S.Y., Kirschner, M.W., 1975. A protein factor essential for microtubule assembly. PNAS 72, 1858-1862. https://doi.org/10.1073/pnas.72.5.1858

Wenzl, C., Yan, S., Laupsien, P., Grosshans, J., 2010. Localization of RhoGEF2 during Drosophila cellularization is developmentally controlled by Slam. Mech. Dev. 127, 371-384. https://doi.org/10.1016/j.mod.2010.01.001

Wilson, P.D., 2011. Apico-basal polarity in polycystic kidney disease epithelia. Biochim. Biophys. Acta 1812, 1239-1248. https://doi.org/10.1016/j.bbadis.2011.05.008

Winder, S.J., Hemmings, L., Maciver, S.K., Bolton, S.J., Tinsley, J.M., Davies, K.E., Critchley, D.R., KendrickJones, J., 1995. Utrophin actin binding domain: analysis of actin binding and cellular targeting. J. Cell. Sci. 108 ( Pt 1), 63-71.

Winkler, F., Gummalla, M., Künneke, L., Lv, Z., Zippelius, A., Aspelmeier, T., Grosshans, J., 2015. Fluctuation analysis of centrosomes reveals a cortical function of kinesin-1. Biophysical journal 109, 856-868.

Wollrab, V., Belmonte, J.M., Baldauf, L., Leptin, M., Nédeléc, F., Koenderink, G.H., 2019. Polarity sorting drives remodeling of actin-myosin networks. J Cell Sci 132, jcs219717.

Woodham, E.F., Machesky, L.M., 2014. Polarised cell migration: intrinsic and extrinsic drivers. Current Opinion in Cell Biology, Cell adhesion and migration 30, 25-32. https://doi.org/10.1016/j.ceb.2014.05.006

Wordeman, L., Mitchison, T.J., 1995. Identification and partial characterization of mitotic centromereassociated kinesin, a kinesin-related protein that associates with centromeres during mitosis. J. Cell Biol. 128, 95-104. https://doi.org/10.1083/jcb.128.1.95

Wu, J., Akhmanova, A., 2017. Microtubule-Organizing Centers. Annual Review of Cell and Developmental Biology 33, 51-75. https://doi.org/10.1146/annurev-cellbio-100616-060615

Xiang, X., 2018. Nuclear movement in fungi. Semin. Cell Dev. Biol. 82, 3-16. https://doi.org/10.1016/j.semcdb.2017.10.024

Yamanaka, T., Horikoshi, Y., Izumi, N., Suzuki, A., Mizuno, K., Ohno, S., 2006. Lgl mediates apical domain disassembly by suppressing the PAR-3-aPKC-PAR-6 complex to orient apical membrane polarity. J. Cell. Sci. 119, 2107-2118. https://doi.org/10.1242/jcs.02938 
Yan, S., Acharya, S., Gröning, S., Gro\s shans, J., 2017. Slam protein dictates subcellular localization and translation of its own mRNA. PLoS biology 15, e2003315.

Yan, S., Großhans, J., 2018. Localization and translation control of slam in Drosophila cellularization. Fly 12, 191-198. https://doi.org/10.1080/19336934.2018.1520574

Yan, S., Lv, Z., Winterhoff, M., Wenzl, C., Zobel, T., Faix, J., Bogdan, S., Grosshans, J., 2013. The F-BAR protein Cip4/Toca-1 antagonizes the formin Diaphanous in membrane stabilization and compartmentalization. J. Cell. Sci. 126, 1796-1805. https://doi.org/10.1242/jcs.118422

Yang, J.T., Saxton, W.M., Goldstein, L.S., 1988. Isolation and characterization of the gene encoding the heavy chain of Drosophila kinesin. Proceedings of the National Academy of Sciences 85, 18641868.

Yu, C.-H., Dang, Y., Zhou, Z., Wu, C., Zhao, F., Sachs, M.S., Liu, Y., 2015. Codon Usage Influences the Local Rate of Translation Elongation to Regulate Co-translational Protein Folding. Molecular Cell 59, 744-754. https://doi.org/10.1016/j.molcel.2015.07.018

Zelhof, A.C., Bao, H., Hardy, R.W., Razzaq, A., Zhang, B., Doe, C.Q., 2001. Drosophila Amphiphysin is implicated in protein localization and membrane morphogenesis but not in synaptic vesicle endocytosis. Development 128, 5005-5015.

Zeng, Y., Cao, Y., Liu, L., Zhao, J., Zhang, T., Xiao, L., Jia, M., Tian, Q., Yu, H., Chen, S., Cai, Y., 2019. SEPT9_i1 regulates human breast cancer cell motility through cytoskeletal and RhoA/FAK signaling pathway regulation. Cell Death Dis 10, 720. https://doi.org/10.1038/s41419-019-1947-9

Zhang, Y., Kong, D., Reichl, L., Vogt, N., Wolf, F., Großhans, J., 2014. The glucosyltransferase Xiantuan of the endoplasmic reticulum specifically affects E-Cadherin expression and is required for gastrulation movements in Drosophila. Dev. Biol. 390, 208-220. https://doi.org/10.1016/j.ydbio.2014.03.007

Zivraj, K.H., Tung, Y.C.L., Piper, M., Gumy, L., Fawcett, J.W., Yeo, G.S.H., Holt, C.E., 2010. Subcellular Profiling Reveals Distinct and Developmentally Regulated Repertoire of Growth Cone mRNAs. J. Neurosci. 30, 15464-15478. https://doi.org/10.1523/JNEUROSCI.1800-10.2010

Zumbrunn, J., Kinoshita, K., Hyman, A.A., Näthke, I.S., 2001. Binding of the adenomatous polyposis coli protein to microtubules increases microtubule stability and is regulated by GSK3 beta phosphorylation. Curr. Biol. 11, 44-49. https://doi.org/10.1016/s0960-9822(01)00002-1

Zwolak, A., Fujiwara, I., Hammer, J.A., Tjandra, N., 2010. Structural Basis for Capping Protein Sequestration by Myotrophin (V-1). J. Biol. Chem. 285, 25767-25781. https://doi.org/10.1074/jbc.M110.135848 


\section{List of figures}

Figure 1 Scheme of actin dynamics and actin-binding proteins in non-muscle cells. .................. 10

Figure 2 Microtubules and actin filaments crosslink in epithelial cells....................................... 13

Figure 3 Asymmetric distribution of cortical components in epithelial cells. ................................. 14

Figure 4 Translocation of Par-3 to anterior by the actomyosin contraction. .............................. 15

Figure 5 Dynamics of cortical domain in Drosophila early embryonic development. .................... 17

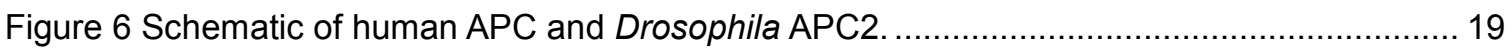

Figure 7 Kinesin-1 localizes to the apical domain during syncytial and cellularization stages...... 22

Figure 8 Khc-Kate localizes to the apical domain during syncytial stage and cellularization........ 23

Figure 9 The furrow invagination is compromised in Kinesin-1 RNAi embryos............................. 24

Figure 10 The nuclear elongation is affected in Kinesin-1 RNAi embryos. ................................... 24

Figure 11 Cortical formation during early embryonic development. ......................................... 25

Figure 12 GFP-Slam is mislocalized during cellularization in Kinesin-1 RNAi embryos. .............. 26

Figure 13 The slam RNA localization is affected in Kinesin-1 RNAi embryos during cellularization.

Figure 14 Slam dynamics are comparable in wild type and Kinesin-1 RNAi embryos at the onset

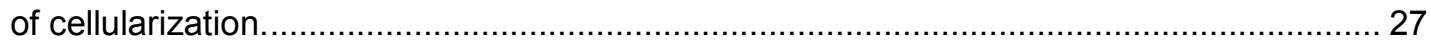

Figure 15 The formation of subapical domain is affected in Kinesin-1 RNAi embryos.................. 28

Figure 16 Subapical domain is influenced in Kinesin-1 RNAi embryos...................................... 29

Figure 17 The distribution of Sponge is affected in Kinesin-1 RNAi embryos during cellularization.

30

Figure 18 The ELMO-GFP localization is affected in Kinesin-1 RNAi embryos. .......................... 31

Figure 19 Centrosomes are not affected in Kinesin-1 RNAi embryos...................................... 33

Figure 20 Minus end direct transport towards centrosome is largely normal in Kinesin-1 RNAi

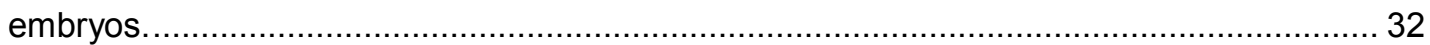

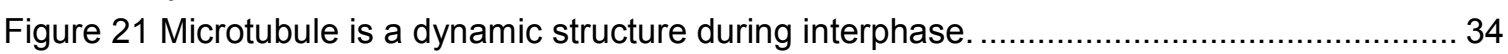

Figure 22 The polymerization of microtubules is normal in Kinesin-1 depleted embryos. ............ 35

Figure 23 EB1 and Moesin mainly localize to the cap domain in wild type embryos. ................... 36

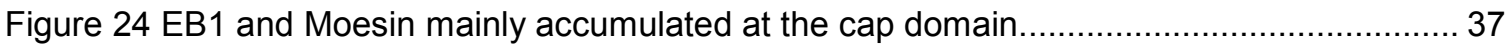

Figure 25 The intercap domain is not affected in Kinesin-1 RNAi embryos. ............................... 38

Figure 26 The differentiation of the cap domain is not influenced in Kinesin-1 RNAi syncytial

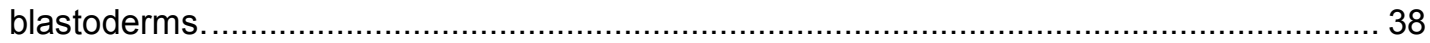

Figure 27 The localization of Utrophin-GFP is influenced in Kinesin-1 RNAi embryos................. 39

Figure 28 Localization of $\mathrm{Cp} \beta$ during syncytial and cellularization stages in wild type embryos... 40

Figure 29 The localization of Cpa is affected in Kinesin-1 RNAi embryos. ............................... 41

Figure 30 The Cpa distribution is disrupted in Kinesin-1 RNAi embryos................................... 42

Figure 31 The Cpa expression is not affected in Kinesin-1 RNAi embryos................................... 43

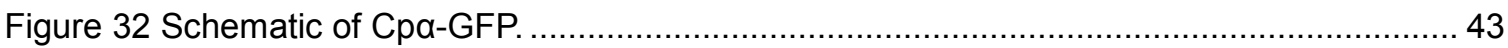

Figure 33 Cpa forms clusters in Drosophila early embryonic development. .............................. 44

Figure 34 Cpa-GFP clusters localize to the cap and intercap domains during the interphase. .... 44

Figure 35 Cpa-GFP clusters move to intercap domain at the onset of cellularization. .................. 45

Figure 36 The Cpa-GFP distribution is dynamic during the interphase...................................... 46

Figure 37 The movement of Cpa-GFP cluster during the interphase ...................................... 47

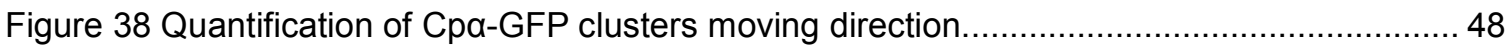

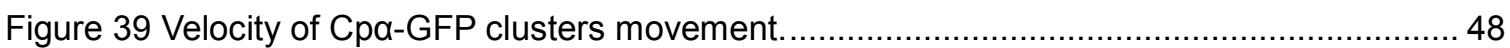

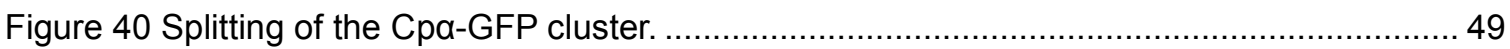


Figure 41 Merging of Cpa-GFP clusters.

Figure 42 The distribution of Cpa-GFP clusters is affected by Kinesin-1 depletion. ..................... 50

Figure 43 The distribution of Cpa-GFP clusters is affected in Kinesin-1 RNAi during the interphase of syncytial blastoderms.

Figure 44 Sqh-GFP is mislocalized at the intercap region in Kinesin-1 RNAi syncytial blastoderms.

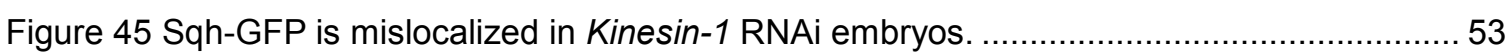

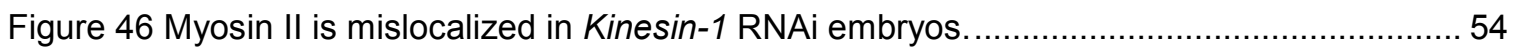

Figure 47 ROCK inhibitor decreases Myosin II activity at the intercap region. ...............................54

Figure 48 The Cpa-GFP distribution is affected by the Y-27632 injection. ..................................5 55

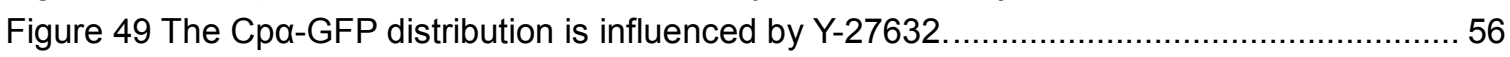

Figure 50 Schematic of Myosin II in F-actin organization in vitro and in vivo.............................. 56

Figure 51 The localization of Dia is affected in Kinesin-1 depleted embryos. ............................. 57

Figure 52 The Cpa-GFP clusters distribution is affected during the syncytial interphase of dia

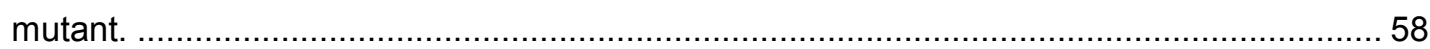

Figure 53 The Cpa distribution is affected in dia mutant embryos during syncytial interphase. ... 59

Figure $54 \mathrm{Dia}$ is required for the distribution of Cpa-GFP clusters during cellularization................60

Figure 55 The Cpa-GFP clusters distribution is affected in dia mutant during cellularization. ...... 61

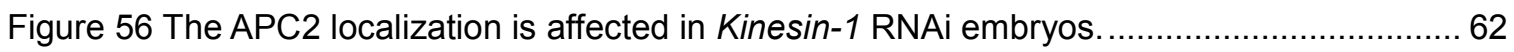

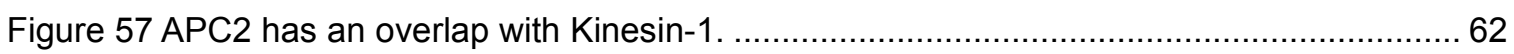

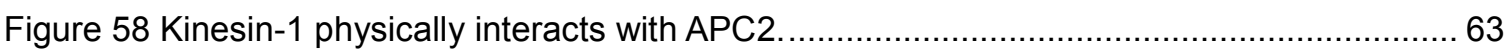

Figure 59 The APC2 protein is changed in different APC2 truncation embryos............................. 64

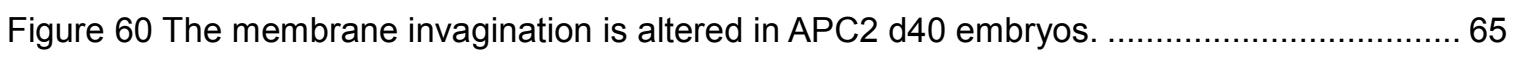

Figure 61 The furrow invagination is affected in APC2 d40 embryos during cellularization.......... 65

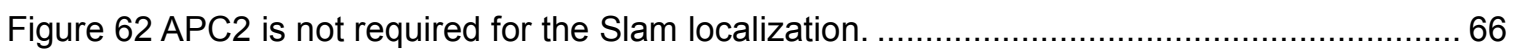

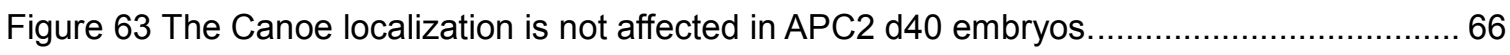

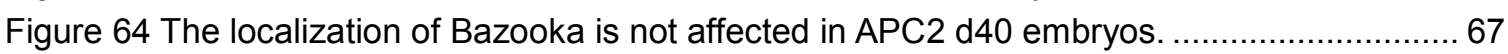

Figure 65 The localization of Dlg is not altered in APC2 d40 embryos..................................... 67

Figure 66 Accumulations of Slam and Amphiphysin at the furrow tip are decreased in APC2 d40

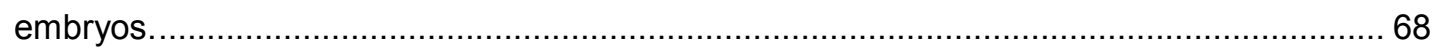

Figure 67 Schematic of the Khc-GFP localization in Drosophila syncytial blastoderms. .............. 71

Figure 68 The formation of cortical domains during Drosophila early embryonic development is

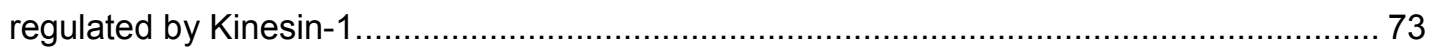

Figure 69 The mislocalization of Cpa affects cap domain contractility. ..................................... 79

Figure 70 Interactions between microtubule and F-actin filament. ........................................... 81

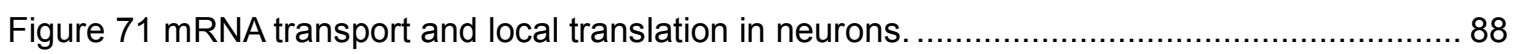

Figure 72 Schematic of Spatio-temporal dynamics of slam mRNA and protein........................... 89

Figure 73 The function of different regions of the slam mRNA sequence in its localization.......... 90

Figure 74 The membrane invagination is affected in slam_fl_acu embryos............................... 92

Figure 75 The Slam protein expression is decreased in slam_fl_acu embryos during

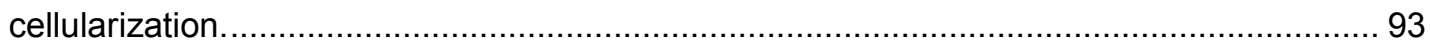

Figure 76 slam mRNA sequence is required for its basal localization during cellularization......... 93

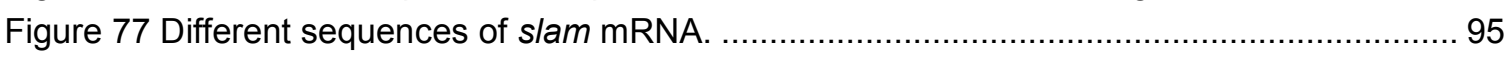

Figure 78 The slam mRNA sequence is required for the membrane invagination. ...................... 96

Figure 79 The membrane invagination is affected in embryos with different hybrid sequences of

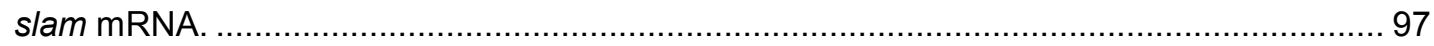

Figure 80 The decreasing of Slam expression in embryos with different hybrid slam mRNA

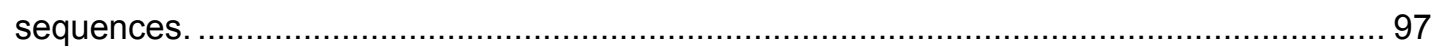

Figure 81 Different hybrid slam mRNA sequences induce various amount of Slam at the basal 
domain.

.98

Figure 82 The slam mRNA sequence is required for the localization of slam mRNA and protein. 99 Figure 83 slam mRNA localization and Slam protein expression level in wild type embryos and embryos with different slam ACU constructs. 100 


\section{List of tables}

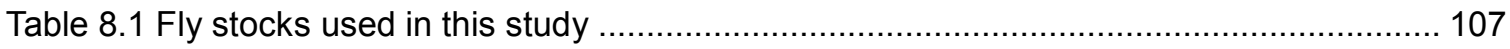

Table 8.2 Fly stocks generated in this study ............................................................ 108

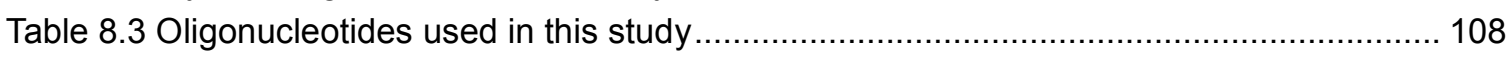

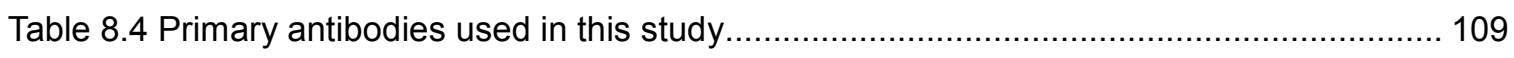

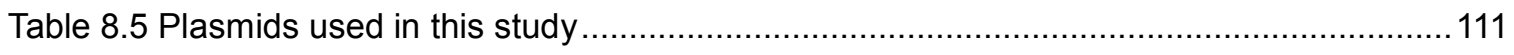

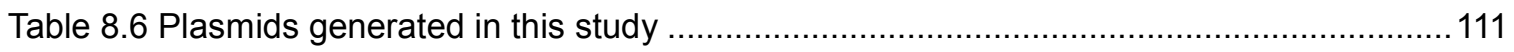

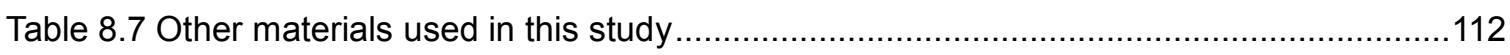

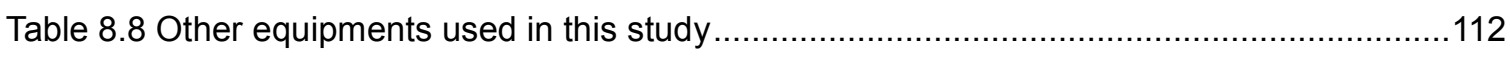

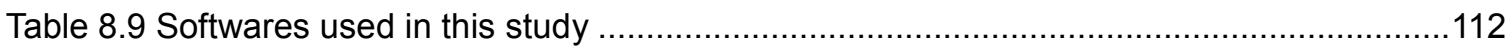

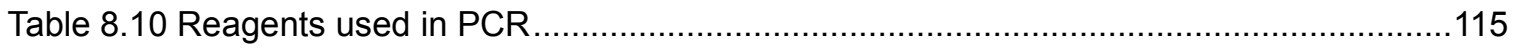

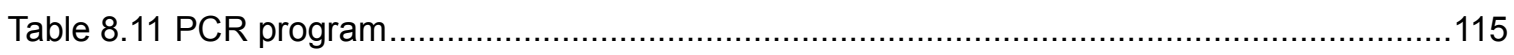




\section{Appendix}

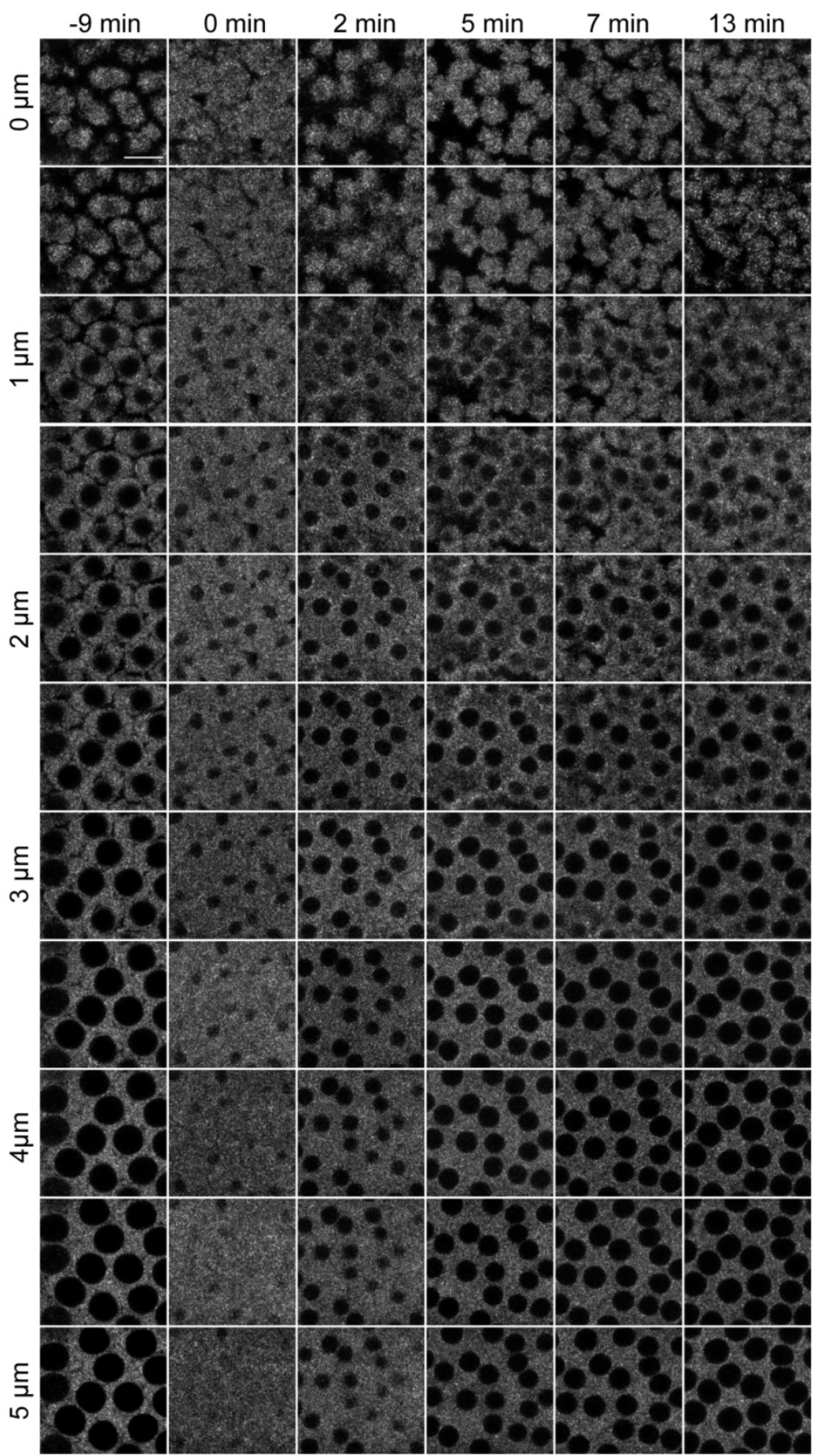

Figure A.1 Khc-Kate accumulates at the cortex of embryos during syncytial and cellularization stages (relevant to Figure 8).

The result shows the localization of Khc-Kate at different depths in embryos during syncytial stage (-9$0 \mathrm{~min}$ ) and cellularization (2-13 min). Z-stack size of each step is $0.5 \mu \mathrm{m}$ and the time interval is $1 \mathrm{~min}$. Scale bar: $10 \mu \mathrm{m}$. 


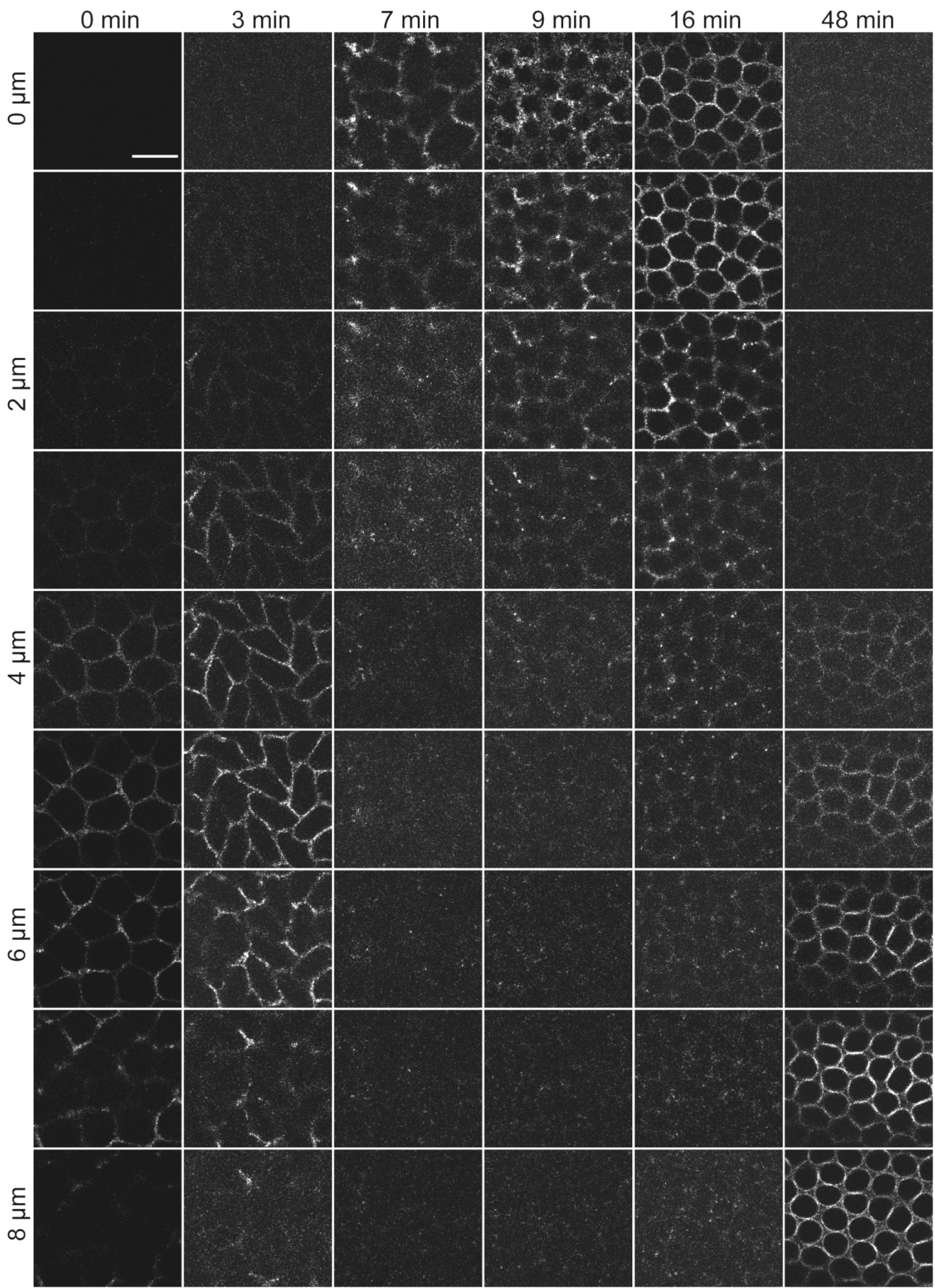

Figure A.2a GFP-Slam localizes to the basal domain in wild type embryos during cellularization (relevant to Figure 12).

The result shows the localization of GFP-Slam during syncytial (0-3 $\mathrm{min}$ ) and cellularization (7-48 $\mathrm{min}$ ) stages. GFP-Slam localizes to the metaphase furrow during the syncytial mitosis and it accumulates to the basal domain during cellularization. Z-stack size of each step is $1 \mu \mathrm{m}$ and the time interval is $1 \mathrm{~min}$. Scale bar: $10 \mu \mathrm{m}$. 


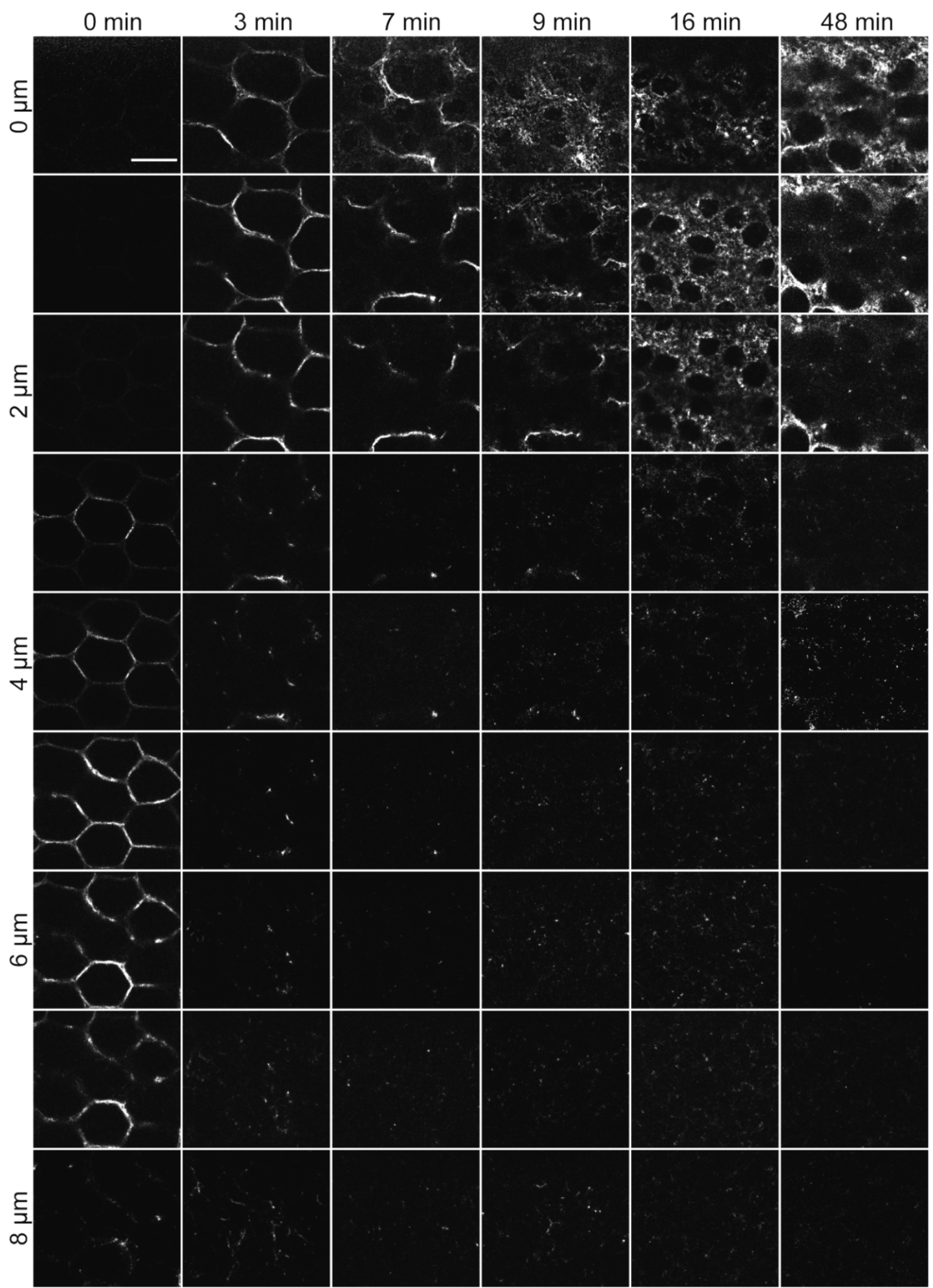

Figure A.2b GFP-Slam is stuck on the surface of Kinesin-1 RNAi embryos (relevant to Figure 12).

The result shows the localization of GFP-Slam during syncytial (0-3 min) and cellularization (7-48 min) stages. GFP-Slam localizes to the metaphase furrow during the syncytial mitosis, while it accumulates at the peripheral side of Kinesin-1 RNAi embryos during cellularization. Z-stack size of each step is 1 $\mu \mathrm{m}$ and the time interval is $1 \mathrm{~min}$. Scale bar: $10 \mu \mathrm{m}$. 


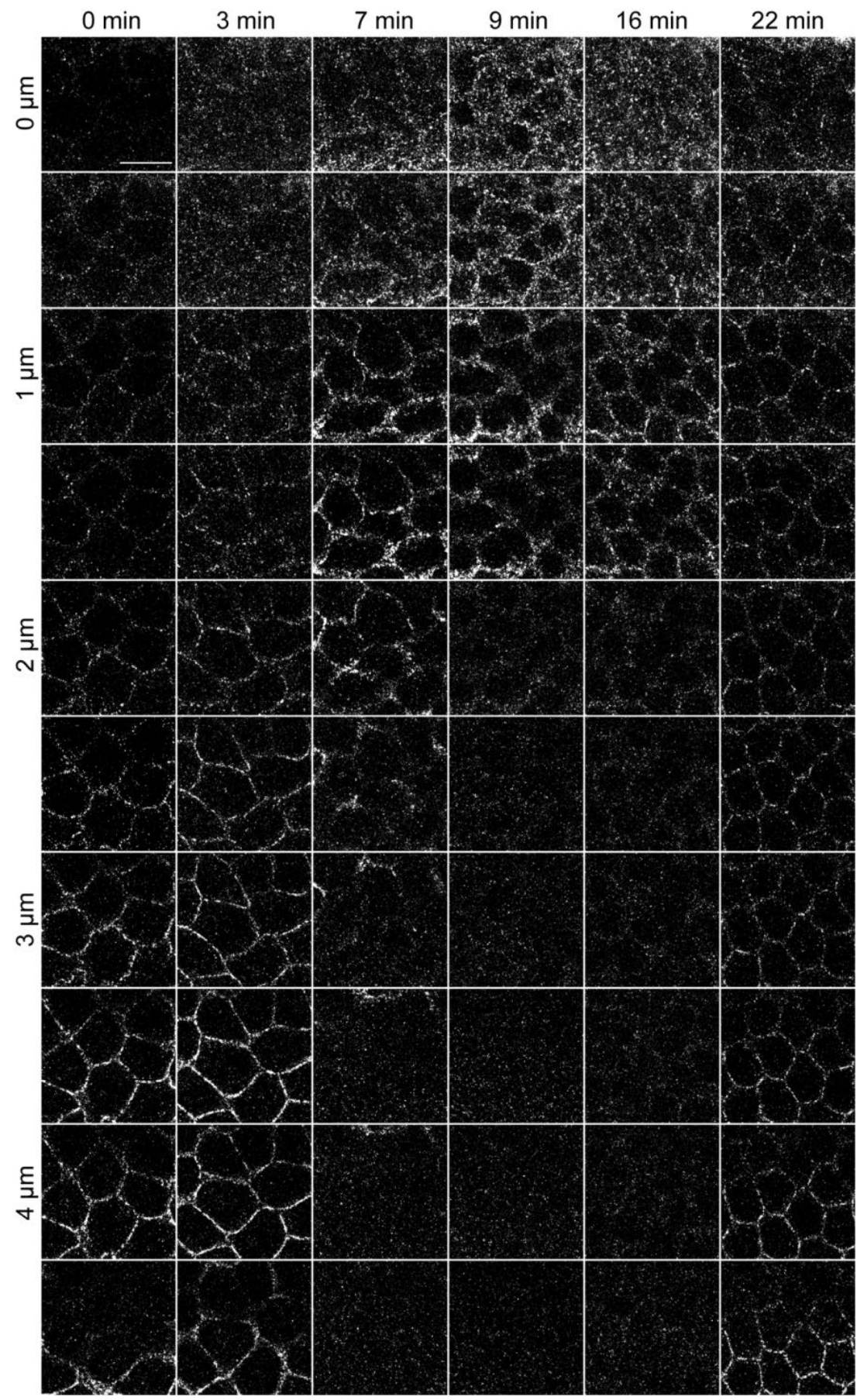

Figure A.3a Canoe-YFP localizes to the subapical domain during cellularization (relevant to Figure 16).

The localization of Canoe-YFP during syncytial stage (0-3 $\mathrm{min}$ ) and cellularization (7-22 $\mathrm{min}$ ). CanoeYFP localizes to the metaphase furrow during syncytial mitosis, Canoe-YFP accumulates to the subapical domain during cellularization. Z-stack size of each step is $0.5 \mu \mathrm{m}$ and the time interval is 1 min. Scale bar: $10 \mu \mathrm{m}$. 


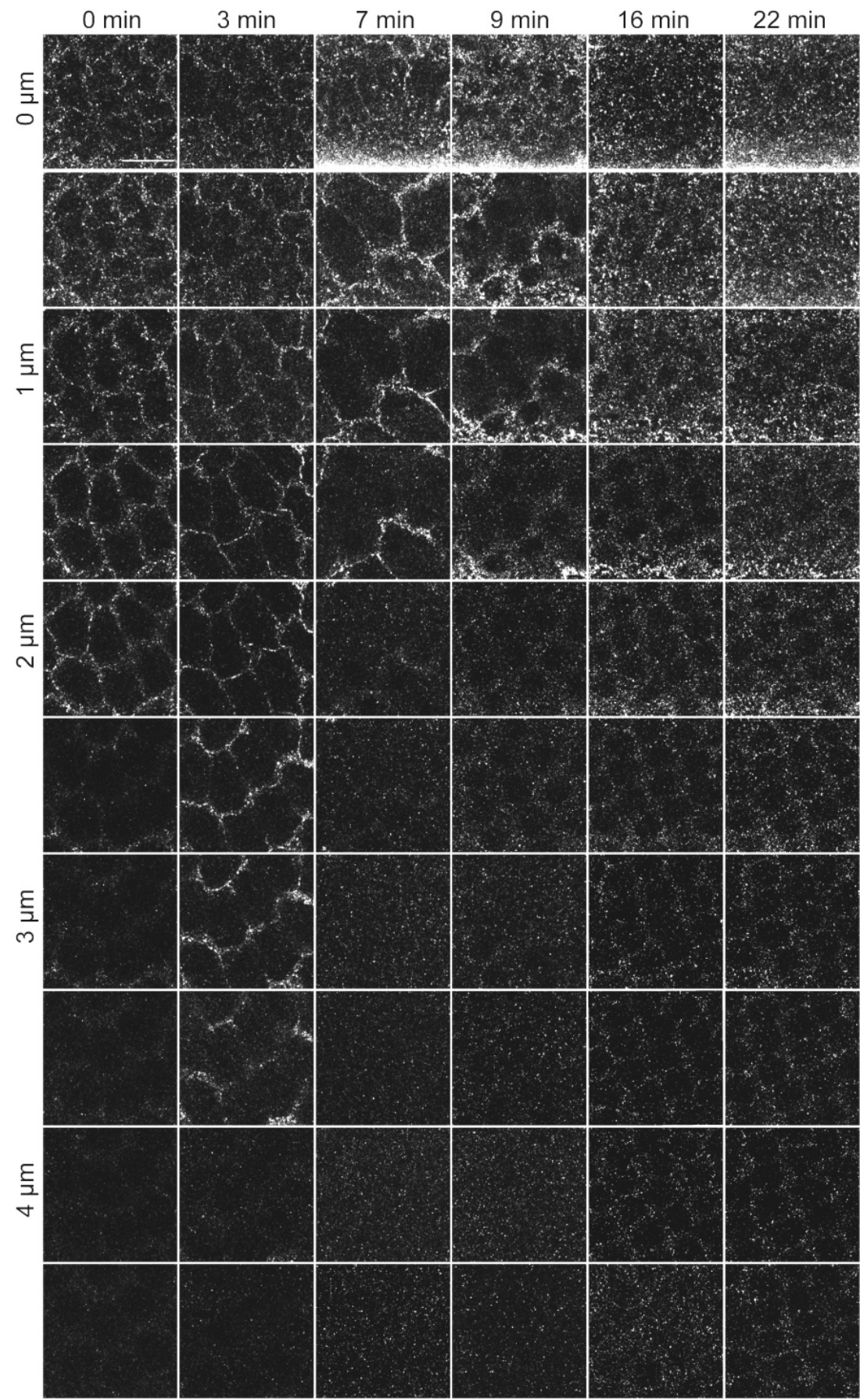

Figure A.3b The subapical accumulation of Canoe-YFP is affected in Kinesin-1 RNAi embryos (relevant to Figure 16).

The result shows the localization of Canoe-YFP during syncytial stage (0-3 $\mathrm{min}$ ) and cellularization (7$22 \mathrm{~min}$ ) in Kinesin-1 RNAi embryos. Canoe-YFP localizes to the metaphase furrow during syncytial mitosis, Canoe-YFP accumulates to the cortex of Kinesin-1 RNAi embryos during cellularization. Zstack size of each step is $0.5 \mu \mathrm{m}$ and the time interval is $1 \mathrm{~min}$. Scale bar: $10 \mu \mathrm{m}$. 


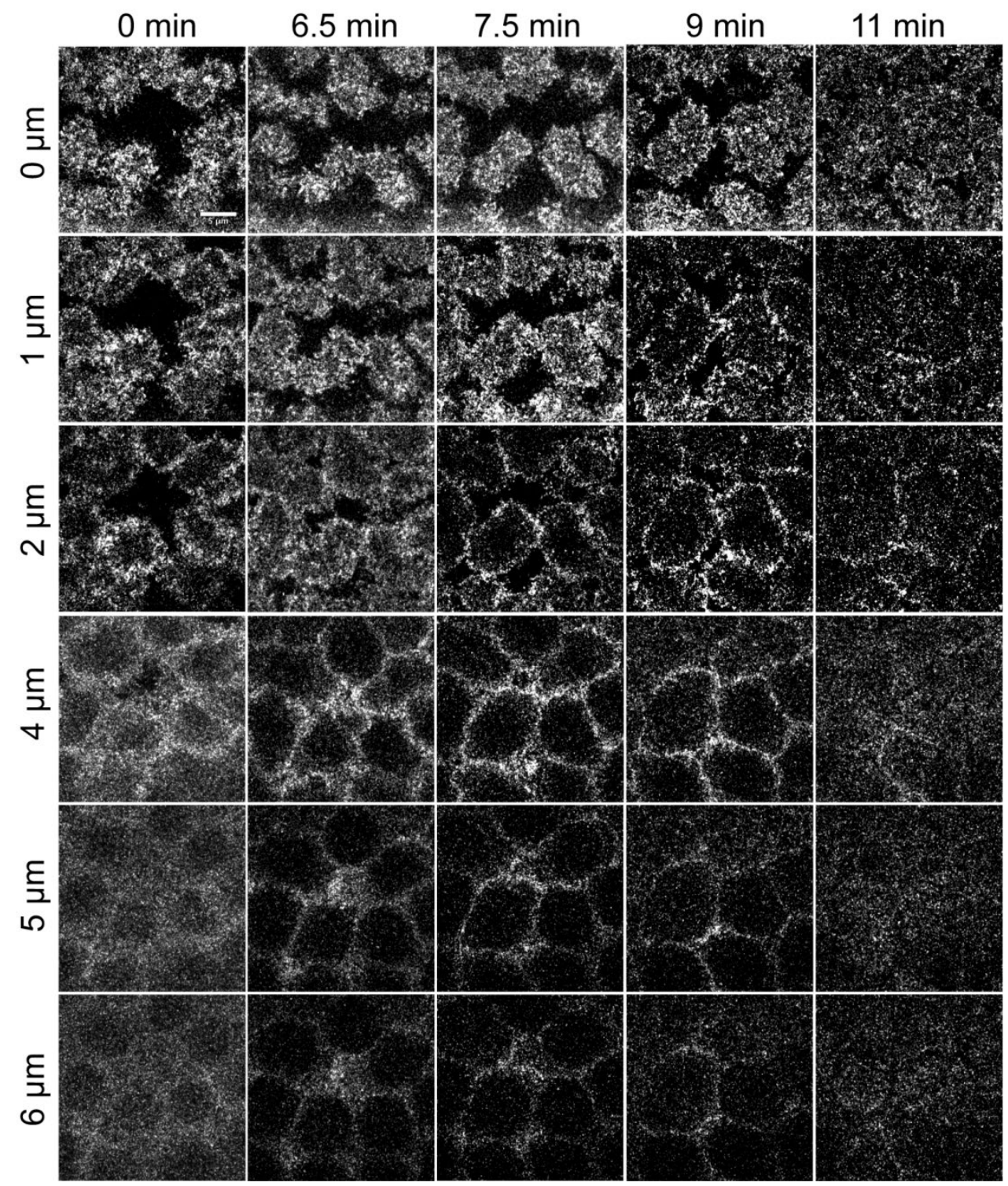

Figure A.4 ELMO-GFP localizes to the intercap domain during the syncytial interphase (relevant to Figure 18).

The result shows the localization of ELMO-GFP in syncytial interphase (0-7.5 min) and mitosis (9-11 min). Z-stack size of each step is $1 \mu \mathrm{m}$ and the time interval is $0.5 \mathrm{~min}$. 


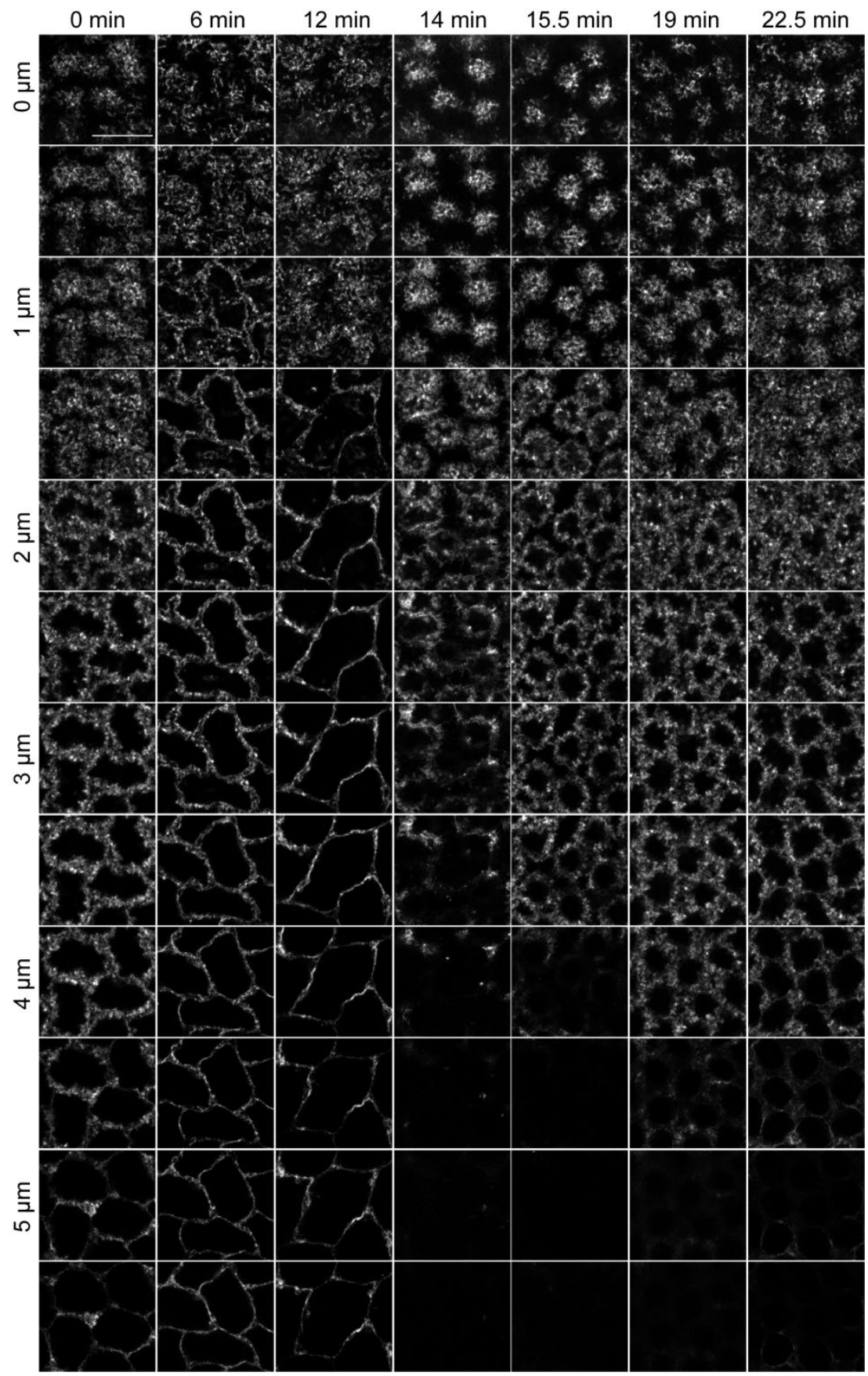

Figure A.5a Utrophin-GFP localizes to the apical domain during the onset of cellularization (relevant to Figure 27).

The result shows the localization of Utrophin-GFP at the syncytial stage (0-12 $\mathrm{min})$ and cellularization (14-22.5 min). During syncytial stage, Utrophin-GFP mainly localizes to the metaphase furrow. At the onset of cellularization, Utrophin-GFP accumulates at the apical domain, Utrophin-GFP migrate with cell membrane at later stage of cellularization. Z-stack size of each step is $0.5 \mu \mathrm{m}$ and the time interval is $1 \mathrm{~min}$. Scale bar: $10 \mu \mathrm{m}$. 


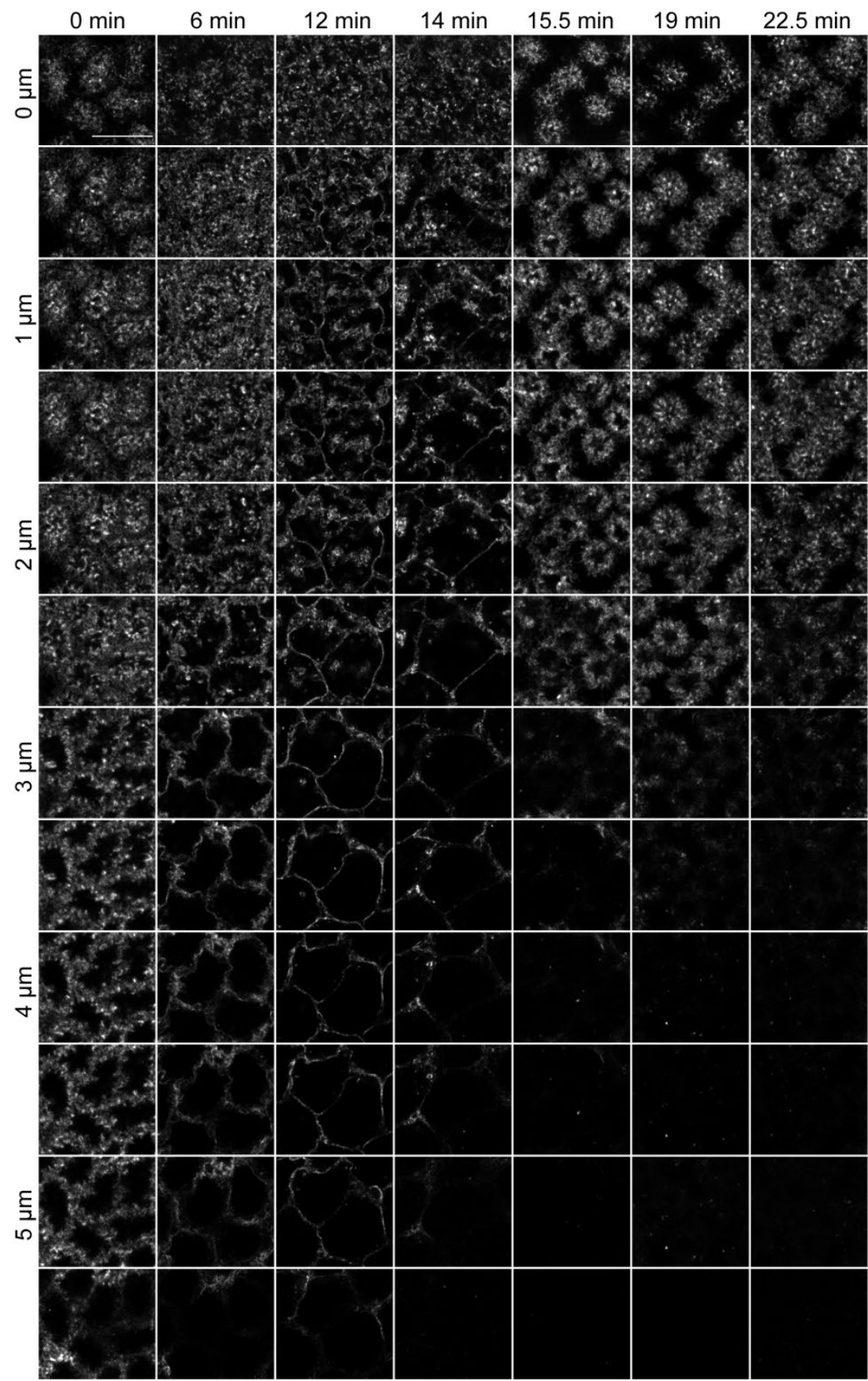

Figure A.5b Utrophin-GFP mainly accumulates to the apical domain during cellularization in Kinesin-1 RNAi embryos (relevant to Figure 27).

The result shows the accumulation of Utrophin-GFP during syncytial stage (0-12 $\mathrm{min})$ and cellularization (14-22.5 min). During syncytial stage, Utrophin-GFP mainly localizes to the metaphase furrow. During cellularization, Utrophin-GFP is stuck at the apical domain. Z-stack size of each step is $0.5 \mu \mathrm{m}$ and the time interval is $1 \mathrm{~min}$. Scale bar: $10 \mu \mathrm{m}$. 


\section{Appendix of statistics}

The nuclear length of wild type and Kinesin-1 RNAi embryos during cellularization. This table is relevant to Figure 10B.

\begin{tabular}{|c|c|c|c|c|}
\hline \multirow{2}{*}{ Time $(\mathrm{min})$} & \multicolumn{2}{|c|}{ Wild type $(\mathrm{N}=3$ embryos) } & \multicolumn{2}{c|}{ Kinesin-1 RNAi (N=3 embryos) } \\
\cline { 2 - 4 } & Mean $(\mu \mathrm{m})$ & SD & Mean $(\mu \mathrm{m})$ & SD \\
\hline 0 & 6.350090 & 0.439589 & 6.733500 & 0.3013014 \\
\hline 4 & 8.362628 & 0.721617 & 7.626400 & 0.5056923 \\
\hline 8 & 9.637343 & 1.175257 & 8.613539 & 0.2224067 \\
\hline 12 & 11.148130 & 1.577557 & 8.928000 & 0.06770035 \\
\hline 16 & 12.670470 & 1.114764 & 10.679830 & 0.8518322 \\
\hline 20 & 13.844160 & 1.141036 & 11.601880 & 1.199810 \\
\hline 24 & 15.014040 & 0.6315665 & & \\
\hline 28 & 15.934650 & 0.2923362 & & \\
\hline
\end{tabular}

The Slam dynamics of wild type and Kinesin-1 RNAi embryos during cellularization. This table is relevant to Figure 14B.

\begin{tabular}{|c|c|c|c|c|}
\hline \multirow[t]{2}{*}{ Time (s) } & \multicolumn{2}{|c|}{ Wild type (N=3 embryos) } & \multicolumn{2}{|c|}{ Kinesin-1 RNAi (N=3 embryos) } \\
\hline & Rel. fluores. int. (a.u.) & SD & Rel. fluores. int. (a.u.) & SD \\
\hline-10. & 3.829533 & 0.5061002 & 9.971203 & 0.049878 \\
\hline 0. & 3.964908 & 0.6025419 & 2.402156 & 0.788729 \\
\hline 10. & 4.103597 & 0.5187289 & 2.706557 & 0.9134315 \\
\hline 20. & 4.188264 & 0.6214462 & 3.055033 & 0.9489852 \\
\hline 30. & 4.154793 & 0.5603987 & 3.250501 & 0.8960351 \\
\hline 40. & 4.294659 & 0.3038435 & 3.177323 & 0.9455963 \\
\hline 50. & 4.321660 & 0.1010913 & 3.253737 & 0.9465262 \\
\hline 60. & 4.611319 & 0.05382587 & 3.485659 & 1.031548 \\
\hline 70. & 4.429471 & 0.2786692 & 3.508861 & 1.215984 \\
\hline 80. & 4.569572 & 0.4979239 & 3.623942 & 1.267833 \\
\hline 90. & 4.612037 & 0.3729164 & 3.542885 & 1.075505 \\
\hline 100. & 4.836527 & 0.2489026 & 3.710381 & 1.054769 \\
\hline 110. & 4.766214 & 0.4513284 & 3.848889 & 1.060606 \\
\hline 120. & 4.636779 & 0.6773341 & 4.054001 & 1.054136 \\
\hline 130. & 4.823813 & 1.006928 & 4.207035 & 1.156050 \\
\hline 140. & 4.796057 & 0.8326656 & 4.212183 & 1.055485 \\
\hline 150. & 4.642243 & 0.6454287 & 3.928543 & 1.086311 \\
\hline 160. & 4.743060 & 0.7149044 & 4.090337 & 1.052874 \\
\hline 170. & 5.017428 & 0.7355981 & 4.220431 & 1.198026 \\
\hline 180. & 5.000250 & 0.6033235 & 4.367489 & 1.056139 \\
\hline 190. & 4.929617 & 0.6935897 & 4.474824 & 0.998392 \\
\hline 200. & 4.984819 & 0.7602426 & 4.431511 & 0.8436043 \\
\hline 210. & 3.829533 & 0.5061002 & 4.532068 & 0.8868408 \\
\hline 220. & 3.964908 & 0.6025419 & 4.639858 & 0.8749656 \\
\hline 230. & 4.103597 & 0.5187289 & 4.553284 & 0.8116309 \\
\hline 240. & 4.188264 & 0.6214462 & 4.665956 & 0.6401311 \\
\hline 250. & 4.154793 & 0.5603987 & 4.653078 & 0.5661798 \\
\hline 260. & 4.294659 & 0.3038435 & 4.470418 & 0.8270344 \\
\hline 270. & 4.321660 & 0.1010913 & 4.671119 & 0.877162 \\
\hline 280. & 4.611319 & 0.05382587 & 4.489297 & 1.034991 \\
\hline 290. & 4.429471 & 0.2786692 & 4.329647 & 0.8077426 \\
\hline 300. & 4.569572 & 0.4979239 & 4.629961 & 1.003110 \\
\hline 310. & 4.612037 & 0.3729164 & 4.568739 & 0.8593271 \\
\hline 320. & 4.836527 & 0.2489026 & 4.787319 & 1.208594 \\
\hline
\end{tabular}


The migration velocity of EB1 in wild type and Kinesin-1 RNAi embryos. This table is relevant to Figure 22B.

\begin{tabular}{|c|c|}
\hline Wild type $(\mu \mathrm{m})$ & Kinesin-1 RNAi $(\mu \mathrm{m})$ \\
\hline 0.2465714 & 0.2652 \\
\hline 0.2618333 & 0.3706 \\
\hline 0.3431667 & 0.3652 \\
\hline 0.2508571 & 0.3300 \\
\hline 0.3971667 & 0.2844 \\
\hline 0.3853333 & 0.2842 \\
\hline 0.3084286 & 0.2606 \\
\hline 0.295400 & 0.2400 \\
\hline
\end{tabular}

EB1 and Moesin fluorescent intensities at the cap domain. This table is relevant to Figure 24B.

\begin{tabular}{|c|c|c|c|c|c|}
\hline \multicolumn{3}{|c|}{ EB1-GFP } & \multicolumn{3}{|c|}{ Moesin-RFP } \\
\hline Inner density $\left(\rho^{\prime}\right)$ & Outer density $\left(\rho^{\circ}\right)$ & Ratio $\left(\rho^{1} / \rho^{0}\right)$ & Inner density $\left(\rho^{\prime}\right)$ & Outer density $\left(\rho^{\circ}\right)$ & Ratio $\left(\rho^{1} / \rho^{0}\right)$ \\
\hline 1020.908 & 317.0827 & 3.21969 & 1013.472 & 332.1269 & 3.05146 \\
\hline 822.338 & 281.6102 & 2.920128 & 786.057 & 224.3275 & 3.50406 \\
\hline 656.983 & 421.7179 & 1.557873 & 705.204 & 210.3784 & 3.352074 \\
\hline 610.629 & 341.5161 & 1.787995 & 996.694 & 322.8125 & 3.087532 \\
\hline 835.207 & 359.8111 & 2.321237 & 1560.743 & 607.4635 & 2.569278 \\
\hline 1074.641 & 297.8138 & 3.608433 & 1723.6 & 610.3652 & 2.823883 \\
\hline 746.102 & 290.005 & 2.572721 & 1597.106 & 627.7129 & 2.544326 \\
\hline 647.939 & 414.9521 & 1.561479 & 1933.901 & 647.5591 & 2.986447 \\
\hline 695.856 & 393.2722 & 1.7694 & 1427.581 & 686.4842 & 2.079554 \\
\hline 693.801 & 337.6774 & 2.054627 & 1701.666 & 732.9671 & 2.321613 \\
\hline & average & 2.337358 & & average & 2.769896 \\
\hline & SD & 0.685807 & & SD & 0.453525 \\
\hline
\end{tabular}

Relative fluorescent intensities of $\mathrm{Cpa}$ and $\mathrm{F}$-actin from the edge of caps in wild type and Kinesin-1 RNAi embryos. This table is relevant to Figure 30A.

\begin{tabular}{|c|c|c|c|c|c|c|c|c|}
\hline \multirow{3}{*}{$\begin{array}{l}\text { Distance } \\
(\mu \mathrm{m})\end{array}$} & \multicolumn{4}{|c|}{ Cpa } & \multicolumn{4}{|c|}{ F-actin } \\
\hline & \multicolumn{2}{|c|}{ Wild type ( $\mathrm{N}=3$ embryos) } & \multicolumn{2}{|c|}{$\begin{array}{c}\text { Kinesin-1 RNAi ( } \mathrm{N}=4 \\
\text { embryos) }\end{array}$} & \multicolumn{2}{|c|}{ Wild type ( $\mathrm{N}=3$ embryos) } & \multicolumn{2}{|c|}{$\begin{array}{c}\text { Kinesin-1 RNAi }(\mathrm{N}=4 \\
\text { embryos) }\end{array}$} \\
\hline & $\begin{array}{l}\text { Rel. fluores. } \\
\text { int. (a.u.) }\end{array}$ & SD & $\begin{array}{l}\text { Rel. fluores. } \\
\text { int. (a.u.) }\end{array}$ & SD & $\begin{array}{l}\text { Rel. } \\
\text { fluores. int. } \\
\text { (a.u.) }\end{array}$ & SD & $\begin{array}{l}\text { Rel. } \\
\text { fluores. int. } \\
\text { (a.u.) }\end{array}$ & SD \\
\hline 0 & 1 & 0 & 1 & 0 & 1 & 0 & 1 & 0 \\
\hline 0.13179 & 0.878565 & 0.06839 & 0.92119 & 0.043957 & 0.928283 & 0.036641 & 0.982716 & 0.007273 \\
\hline 0.26358 & 0.727335 & 0.071219 & 0.829384 & 0.094867 & 0.855246 & 0.027306 & 0.959262 & 0.021768 \\
\hline 0.39536 & 0.628983 & 0.075185 & 0.790128 & 0.089557 & 0.834213 & 0.050666 & 0.909895 & 0.008595 \\
\hline 0.52715 & 0.542008 & 0.075456 & 0.765303 & 0.088006 & 0.820246 & 0.059092 & 0.854434 & 0.008533 \\
\hline 0.65894 & 0.495732 & 0.048728 & 0.726853 & 0.139613 & 0.779002 & 0.042918 & 0.814307 & 0.023689 \\
\hline 0.79073 & 0.486769 & 0.050787 & 0.686049 & 0.13375 & 0.755942 & 0.023816 & 0.77033 & 0.006985 \\
\hline 0.92252 & 0.469225 & 0.057831 & 0.712531 & 0.138901 & 0.730493 & 0.039731 & 0.716589 & 0.013203 \\
\hline 1.05431 & 0.471994 & 0.055199 & 0.686937 & 0.090492 & 0.68284 & 0.039353 & 0.675619 & 0.017278 \\
\hline 1.18609 & 0.480245 & 0.057396 & 0.648616 & 0.062001 & 0.662091 & 0.042633 & 0.620848 & 0.004524 \\
\hline 1.31788 & 0.462036 & 0.057006 & 0.625296 & 0.077066 & 0.64325 & 0.0215 & 0.61054 & 0.01434 \\
\hline 1.44967 & 0.461839 & 0.072181 & 0.613827 & 0.091447 & 0.63452 & 0.01534 & 0.60923 & 0.011235 \\
\hline
\end{tabular}


Relative fluorescent intensities of $\mathrm{Cp} \alpha$ and $\mathrm{F}$-actin at the cap region in wild type and Kinesin-1 RNAi embryos. This table is relevant to Figure 30B.

\begin{tabular}{|c|c|c|c|}
\hline \multicolumn{2}{|c|}{ Wild type (Fluorescent intensity at the cap region) } & Kinesin-1 RNAi (Fluorescent intensity at the cap region) \\
\hline Cpa(a.u.) & F-actin (a.u.) & Cpa(a.u.) & F-actin (a.u.) \\
\hline 0.879588 & 1.055885 & 0.8679479 & 0.5064458 \\
\hline 0.955298 & 1.15431 & 0.95118 & 0.4732331 \\
\hline 0.889619 & 1.161678 & 0.9748557 & 0.414373 \\
\hline 1.087595 & 1.059683 & 0.8939785 & 0.4712038 \\
\hline 0.939851 & 1.175757 & 0.9165654 & 0.4300577 \\
\hline 0.860613 & 1.105844 & 0.8299149 & 0.5239398 \\
\hline 1.034852 & 1.047098 & 0.9073483 & 0.5650458 \\
\hline 1.075269 & 1.118556 & 0.8169756 & 0.5560896 \\
\hline 1.051973 & 0.8957004 & 0.9234782 & 0.5692525 \\
\hline 1.012733 & 0.8667325 & 0.9405702 & 0.6497908 \\
\hline 0.99369 & 0.8612377 & 0.8704801 & 0.6517811 \\
\hline 1.118229 & 0.8359668 & 0.8990428 & 0.6557164 \\
\hline 1.058182 & 0.8676694 & 0.9088929 & 0.6478005 \\
\hline 1.044329 & 0.9329737 & 0.8265725 & 0.4575144 \\
\hline 1.017279 & 1.052796 & 0.7896029 & \\
\hline 1.074895 & 0.8887623 & 0.8002887 & \\
\hline 0.94866 & 0.8974983 & 0.8032513 & \\
\hline 0.957345 & 0.8794186 & 0.9364429 & \\
\hline & 1.030766 & 0.9196039 & \\
\hline & & 1.095969 & \\
\hline & & 1.045427 & \\
\hline & & 1.037704 & 1.1102 \\
\hline
\end{tabular}

The Cpa-GFP distribution during the interphase. This table is relevant to Figure 36B.

\begin{tabular}{|c|c|}
\hline 13th early interphase $(0 \mathrm{~s})\left(\rho^{\circ} / \rho^{i}\right)$ & 13th later interphase $(120 \mathrm{~s})\left(\rho^{\circ} / \rho^{\prime}\right)$ \\
\hline 1.005717 & 5.632417 \\
\hline 2.212577 & 2.765722 \\
\hline 1.005717 & 3.017151 \\
\hline 0.8045735 & 6.034302 \\
\hline 1.173336 & 4.525726 \\
\hline 0.1676195 & 1.609147 \\
\hline 1.340956 & 1.760005 \\
\hline 1.173336 & 6.034302 \\
\hline 0.005717 & 2.514292 \\
\hline 1.005717 & 4.525726 \\
\hline 2.011434 & 9.051453 \\
\hline 0.6285731 & 6.034302 \\
\hline 0.9142881 & 4.525726 \\
\hline 1.005717 & 3.687629 \\
\hline 0.8620431 & 2.514292 \\
\hline 1.206860 & 4.525726 \\
\hline 1.005717 & 7.040019 \\
\hline 0.7040018 & 3.520009 \\
\hline 2.011434 & 1.508575 \\
\hline 0.4225359 & 5.028584 \\
\hline 1.072865 & 3.424166 \\
\hline 1.220182 & 5.632417 \\
\hline 1.321455 & 2.765722 \\
\hline 0.6023944 & \\
\hline 0.5870674 & \\
\hline
\end{tabular}

Measurement of Cpa-GFP clusters density of the inner $\left(\rho^{\prime}\right)$ and outer $\left(\rho^{\circ}\right)$ cap region in 2 min.. 
Cpa-GFP clusters moving direction. This table is relevant to Figure 38B.

\begin{tabular}{|c|c|c|c|c|c|c|c|c|c|c|c|c|c|}
\hline Angle & $40-50$ & $60-70$ & $70-80$ & $80-90$ & $90-$ & $100-$ & $110-$ & $120-$ & $130-$ & $140-$ & $150-$ & $160-$ & $170-$ \\
& & & & & 100 & 110 & 120 & 130 & 140 & 150 & 160 & 170 & 180 \\
\hline Number & 1 & 1 & 3 & 6 & 4 & 1 & 4 & 4 & 5 & 7 & 6 & 4 & 6 \\
\hline
\end{tabular}

Velocity of Cpa-GFP clusters movement. This table is relevant to Figure 39.

\begin{tabular}{|c|c|c|c|c|c|c|c|c|c|c|c|c|c|}
\hline $\begin{array}{c}\text { Velocity } \\
(\mu \mathrm{m} / \mathrm{s})\end{array}$ & 0.01 & 0.02 & 0.03 & 0.04 & 0.05 & 0.07 & 0.06 & 0.08 & 0.09 & 0.1 & 0.11 & 0.12 & 0.13 \\
\hline Number & 2 & 8 & 8 & 8 & 13 & 9 & 6 & 6 & 2 & 4 & 3 & 1 & 1 \\
\hline
\end{tabular}

Cpa-GFP clusters index during the interphase in wild type and Kinesin-1 RNAi embryos. This table is relevant to Figure 43.

\begin{tabular}{|c|c|c|c|}
\hline \multicolumn{2}{|c|}{ Wild type $\left(\rho^{\circ} / \rho^{i}\right)$} & \multicolumn{2}{|c|}{ Kinesin-1 RNAi $\left(\rho^{\circ} / \rho^{i}\right)$} \\
\hline early stage (0 min) & later stage (2 $\mathrm{min})$ & early stage (0 min) & later stage (2 $\mathrm{min})$ \\
\hline 0.571833 & 2.347556 & 1.229789 & 1.450661 \\
\hline 1.140855 & 3.399762 & 1.096252 & 0.130112 \\
\hline 0.809733 & 2.993557 & 0.898281 & 0.322316 \\
\hline 1.23462 & 1.894044 & 1.158399 & 0.331652 \\
\hline 1.067193 & 1.985734 & 0.834842 & 0.300919 \\
\hline 0.84878 & 1.172113 & 0.772611 & 0.602287 \\
\hline 1.429628 & 2.172547 & 0.765104 & 0.518006 \\
\hline 0.868239 & 2.296976 & 0.930176 & 0.501785 \\
\hline 0.94683 & 5.523145 & 0.87804 & 0.386377 \\
\hline 0.865456 & 1.366684 & 0.842366 & 0.46145 \\
\hline 1.639438 & 1.279845 & 0.702924 & 0.194468 \\
\hline \multirow[t]{9}{*}{1.056231} & 2.591723 & 0.907798 & 0.684253 \\
\hline & & 0.945398 & 0.237814 \\
\hline & & 0.874935 & 0.402471 \\
\hline & & & 0.345758 \\
\hline & & & 0.311639 \\
\hline & & & 0.41793 \\
\hline & & & 0.404759 \\
\hline & & & 0.395758 \\
\hline & & & 0.351797 \\
\hline
\end{tabular}

Relative fluorescent intensities of F-actin and Cpa-GFP from the cap edge in embryos with or without Y-27632 injection. This table is relevant to Figure 49A.

\begin{tabular}{|c|c|c|c|c|c|c|c|c|}
\hline \multirow{3}{*}{$\begin{array}{c}\text { Distance } \\
(\mu \mathrm{m})\end{array}$} & \multicolumn{4}{|c|}{ Cpa (4 embryos) } & \multicolumn{4}{|c|}{ F-actin (4 embryos) } \\
\hline & \multicolumn{2}{|c|}{$-Y-27632$} & \multicolumn{2}{|c|}{$+Y-27632$} & \multicolumn{2}{|c|}{$-Y-27632$} & \multicolumn{2}{|c|}{$+Y-27632$} \\
\hline & $\begin{array}{l}\text { Rel. fluores. } \\
\text { int. (a.u.) }\end{array}$ & SD & $\begin{array}{l}\text { Rel. fluores. } \\
\text { int. (a.u.) }\end{array}$ & SD & $\begin{array}{l}\text { Rel. fluores. } \\
\text { int. (a.u.) }\end{array}$ & SD & $\begin{array}{c}\text { Rel. fluores. } \\
\text { int. (a.u.) }\end{array}$ & SD \\
\hline 0 & 1 & 0 & 1 & 0 & 1 & 0 & 1 & 0 \\
\hline 0.06589 & 0.996007 & 0.017533 & 0.981081 & 0.0191 & 0.999932 & 0.026163 & 0.986033 & 0.004483 \\
\hline 0.13179 & 0.97977 & 0.040542 & 0.959663 & 0.034552 & 0.991803 & 0.039882 & 0.978524 & 0.012838 \\
\hline 0.19768 & 0.952555 & 0.058456 & 0.939977 & 0.048054 & 0.968636 & 0.047245 & 0.961003 & 0.014737 \\
\hline 0.26358 & 0.924138 & 0.071194 & 0.921348 & 0.059456 & 0.94696 & 0.05914 & 0.944181 & 0.025163 \\
\hline 0.32947 & 0.89446 & 0.078456 & 0.909132 & 0.064073 & 0.917763 & 0.060156 & 0.915505 & 0.024292 \\
\hline 0.39536 & 0.867228 & 0.079129 & 0.898247 & 0.069926 & 0.889361 & 0.057876 & 0.886286 & 0.020879 \\
\hline 0.46126 & 0.836951 & 0.074499 & 0.890702 & 0.071415 & 0.861132 & 0.053766 & 0.85721 & 0.021076 \\
\hline 0.52715 & 0.812574 & 0.075303 & 0.881813 & 0.073533 & 0.829925 & 0.0381 & 0.828167 & 0.026252 \\
\hline 0.59305 & 0.79075 & 0.083365 & 0.868233 & 0.078952 & 0.801713 & 0.034018 & 0.797765 & 0.030094 \\
\hline
\end{tabular}


Exponential decay of Cpa-GFP and F-actin with or without Y-27632. This table is relevant to Figure 49B.

\begin{tabular}{|c|c|c|c|}
\hline \multicolumn{2}{|c|}{ Cpa } & \multicolumn{2}{c|}{ F-actin } \\
\hline- Y-27632 & $\mathbf{+ Y - 2 7 6 3 2}$ & $\mathbf{- Y - 2 7 6 3 2}$ & $\mathbf{+ Y - 2 7 6 3 2}$ \\
\hline 0.2949 & 0.6874 & 2.506 & 1.042 \\
\hline 0.3693 & 0.8092 & 1.033 & 2.474 \\
\hline 0.2415 & 0.6095 & 1.888 & 1.989 \\
\hline 0.4568 & 0.6068 & 1.656 & 1.855 \\
\hline 0.375 & 0.9636 & 1.785 & 1.426 \\
\hline 0.3809 & 1.316 & 1.387 & 1.476 \\
\hline \multicolumn{3}{|c}{} \\
\hline
\end{tabular}

The distribution of Cpa clusters in 2 min in wild type and dia mutant embryos. This table is relevant to Figure 53B.

\begin{tabular}{|c|c|c|c|}
\hline \multicolumn{2}{|c|}{ Wild type $\left(\rho^{\%} / \rho^{i}\right)$} & \multicolumn{2}{c|}{ Kinesin-1 RNAi $\left(\rho^{\circ} / \rho^{i}\right)$} \\
\hline early stage $(0 \mathrm{~min})$ & later stage $(2 \mathrm{~min})$ & early stage $(0 \mathrm{~min})$ & 0.424753 \\
\hline 1.180040 & 2.326675 & 0.7471859 & 0.1006008 \\
\hline 0.5472848 & 1.070338 & 0.7223611 & 0.6207907 \\
\hline 0.7478014 & 3.371664 & 0.9873147 & 0.3664053 \\
\hline 0.6865696 & 1.997717 & 1.063170 & 0.5768253 \\
\hline 0.7107844 & 2.645112 & 0.8212001 & 0.5017021 \\
\hline 1.077478 & 2.313154 & 0.4622549 & 0.5406291 \\
\hline 0.8386269 & 3.408427 & 0.7085866 & 0.6050097 \\
\hline 1.161476 & 2.641629 & 1.388973 & 0.2542119 \\
\hline 0.4586613 & 4.733204 & 0.8360123 & 0.4486314 \\
\hline 1.410769 & 3.107769 & 1.170239 & 0.840777 \\
\hline 0.8145624 & 2.159455 & 1.629714 & 0.3369865 \\
\hline 0.8605869 & 3.371313 & 1.116712 & 0.3649263 \\
\hline & 1.848353 & 0.6073442 & 0.9647123 \\
\hline & 2.135873 & 0.8584544 & 0.818248 \\
\hline & 2.773168 & & 0.5225419 \\
\hline & 2.002797 & & 0.392579 \\
\hline & 1.573267 & & 0.423446 \\
\hline & & & 0.3410224 \\
\hline & & & 0.4752006 \\
\hline & & & 0.254428 \\
\hline & & & 0.3392936 \\
\hline & & & 0.3332689 \\
\hline & & & 0.1684013 \\
\hline
\end{tabular}

The furrow length in wild type and APC2 d40 embryos during cellularization. This table is relevant to Figure 61.

\begin{tabular}{|c|c|c|c|c|}
\hline \multirow{2}{*}{ Time (min) } & \multicolumn{2}{|c|}{ Wild tpye (3 embryos) } & \multicolumn{2}{c|}{ APC2 d40 (3 embryos) } \\
\cline { 2 - 5 } & Mean $(\boldsymbol{\mu})$ & SD & \multicolumn{2}{c|}{ Mean $(\boldsymbol{\mu m})$} \\
\hline 0 & 0.000000 & 0.3226639 & 0.000000 & 0.116252 \\
\hline 6 & 1.291689 & 0.7103117 & 0.9188303 & 0.271961 \\
\hline 12 & 2.208789 & 0.156609 & 1.361214 & 0.2159424 \\
\hline 20 & 3.694231 & 0.07750136 & 1.823665 & 0.3779119 \\
\hline 34 & 6.951504 & 1.248934 & 4.612493 & 0.700544 \\
\hline 42 & 11.096100 & 1.637062 & 6.499612 & 0.2919958 \\
\hline
\end{tabular}


The accumulations of Slam and Amphiphysin at the furrow in wild type and APC2 d40 embryos. This table is relevant to Figure 66B.

\begin{tabular}{|c|c|c|c|}
\hline \multicolumn{2}{|c|}{ Wild type (Fluores. int.) } & \multicolumn{2}{|c|}{ APC2 d40 (Fluores. int.) } \\
\hline Slam (a.u.) & Amphiphysin (a.u.) & Slam (a.u.) & Amphiphysin (a.u.) \\
\hline 57.048 & 47.78 & 21.505 & 33.595 \\
\hline 51.708 & 45.97 & 26.768 & 34.03 \\
\hline 56.206 & 45.201 & 24.387 & 33.993 \\
\hline 54.937 & 51.614 & 22.919 & 34.6 \\
\hline 55.53 & 46.224 & 21.507 & 36.535 \\
\hline 54.722 & 52.239 & 26.741 & 35.03 \\
\hline 57.833 & 48.708 & 22.185 & 34.278 \\
\hline 53.981 & 44.357 & 21.264 & 34.299 \\
\hline 53.389 & 46.118 & 18.401 & 35.158 \\
\hline 53.229 & 44.856 & 24.537 & 37.484 \\
\hline 55.842 & 51.896 & 25.271 & 36.158 \\
\hline 57.243 & 47.725 & 26.458 & 37.257 \\
\hline 53.644 & 43.343 & 26.026 & 38.449 \\
\hline 52.037 & 46.732 & 25.468 & 35.826 \\
\hline 51.875 & 50.167 & 21.174 & 36.164 \\
\hline 56.569 & 42.977 & 20.664 & 35.908 \\
\hline 52.595 & 46.62 & 20.866 & 36.745 \\
\hline 51.523 & 45.398 & 21.653 & 37.621 \\
\hline 52.019 & 51.192 & 20.88 & 36.729 \\
\hline 54.123 & 48.451 & 23.776 & 38.342 \\
\hline 55.03 & 48.042 & 23.306 & 38.588 \\
\hline 53.623 & 48.754 & 23.84 & 35.775 \\
\hline 55.755 & 43.632 & 23.861 & 32.354 \\
\hline 54.567 & 50.713 & 26.732 & 28.968 \\
\hline 57.447 & 47.504 & 24.752 & 31.354 \\
\hline 57.391 & 51.405 & 22.132 & 30.111 \\
\hline 51.194 & 52.269 & 25.993 & 34.623 \\
\hline 56.516 & 46.674 & 25.759 & 29.301 \\
\hline 55.741 & 48.356 & 23.322 & 30.192 \\
\hline 52.051 & 51.63 & 16.329 & 31.04 \\
\hline 54.505 & 46.083 & 17.106 & 33.785 \\
\hline & 54.776 & 17.85 & 29.738 \\
\hline & 44.766 & 21.037 & \\
\hline
\end{tabular}

The membrane invagination in wild type and slam_fl_acu embryos. This table is relevant to Figure 74.

\begin{tabular}{|c|c|c|}
\hline Time (min) & Wild type $(\mu \mathrm{m})$ & slam_fl_acu $(\mu \mathrm{m})$ \\
\hline 0 & 0 & 0 \\
\hline 10 & 2.377 & 1.96533 \\
\hline 20 & 7.338333333 & 3.823 \\
\hline 30 & 16.22366667 & 7.653 \\
\hline 40 & 35.237 & 8.34967 \\
\hline
\end{tabular}

The furrow length variation of different slam mRNA sequences. This table is relevant to Figure 78.

\begin{tabular}{|c|c|c|c|c|}
\hline Wild type $(\mu \mathrm{m})$ & slam_fl_acu ( $\mu \mathrm{m})$ & slam_acu1 ( $\mu \mathrm{m})$ & slam_acu2 ( $\mu \mathrm{m})$ & slam_acu3 $(\mu \mathrm{m})$ \\
\hline 31.73625 & 33.82875 & 15.81 & 11.679 & 33.41 \\
\hline 32.89875 & 13.48907 & 14.88 & 8.68 & 10.85 \\
\hline 26.97 & 16.585 & 37.212 & 9.772 & 16.433 \\
\hline \multirow[t]{10}{*}{29.87625} & 16.74477 & 15.812 & 30.38 & 22.63 \\
\hline & 19.18125 & 31.86 & 8.37 & 5.58 \\
\hline & 23.71616 & 32.55 & 8.99 & 15.5 \\
\hline & 20.80875 & 28.38 & 6.827 & 7.446 \\
\hline & 13.95 & 30.688 & 11.47 & 9.92 \\
\hline & 19.06645 & 14.417 & 11.47 & 23.87 \\
\hline & 24.52991 & 27.898 & 10.54 & 22.01 \\
\hline & 11.50875 & 31.386 & 6.045 & 20.5781 \\
\hline & 32.43375 & 17.439 & 8.99 & 12.6728 \\
\hline & & & & 13.8857 \\
\hline
\end{tabular}


Different sequences of slam ACU induce the various amount of Slam at the basal domain. This table is relevant to Figure 80 .

\begin{tabular}{|c|c|c|c|c|c|}
\hline Wild type (a.u.) & slam_acu1 (a.u.) & slam_acu2 (a.u.) & slam_acu3 (a.u.) & BamH_acu (a.u.) & slam_fl_acu (a.u.) \\
\hline 3.98897 & 6.952833 & 8.599084 & 1.278049 & 9.444171 & 0.494861 \\
\hline 5.76683 & 4.368125 & 0.725837 & 3.400146 & 8.740081 & 3.740347 \\
\hline 5.72460 & 6.007708 & 6.248769 & 4.790305 & 4.747544 & 8.919889 \\
\hline 5.13760 & 3.818000 & 4.063657 & 3.969512 & 0.866967 & 1.308819 \\
\hline 5.66361 & 4.185521 & 6.705833 & 0.466293 & 4.242292 & 5.618111 \\
\hline 3.72720 & 1.965625 & 0.805260 & 1.031524 & 2.717620 & 1.248889 \\
\hline 5.62362 & 5.262396 & 7.737895 & 0.465793 & 3.732280 & 1.316056 \\
\hline 3.87202 & 3.182750 & 3.118994 & 8.014146 & 1.060214 & 4.625056 \\
\hline & 3.105972 & 4.831557 & 3.504976 & 6.232857 & \\
\hline & 1.216979 & 2.516639 & 10.003210 & 4.812136 \\
\hline
\end{tabular}




\section{Abbreviations}

bp: base pairs

DAPI: 4',6'-Diamidino-2-phenylindole

$\mathrm{ddH}_{2} \mathrm{O}$ : double distilled water

${ }^{\circ} \mathrm{C}$ : degree Celsius

DNA: deoxyribonucleic acid

EDTA: ethylenediaminetetraacetic acid

FRT: flippase recognition targer

FRAP: fluorescence recovery after photobleaching

GFP: green fluorescent protein

g: gram

h: hour

KD: kiloDalton

I: litre

M: mole

m: milli

nt: nucleotide

$\mu$ : micro

min: minute

s: second

PBS: Phosphate buffered saline

PCR: polymerase chain reaction

RNA: ribonucleic acid

RNAi: RNA interference

rpm: revolutions per minute

$\mathrm{RT}$ : room temperature

SDS: sodiumdodecylsulphate

SDS-PAGE: SDS-polyacylamide gel electrophoresis

Tris: tris(hydroxymethyl) aminomethne hydrochloride

$\mathrm{Ca}^{2+}:$ calcium ion

$\mathrm{Mg}^{2+}:$ magnesium ion 